\title{
Broadband and statistical characterization of echoes from random scatterers: application to acoustic scattering by marine organisms
}

\author{
by \\ Wu-Jung Lee \\ B.S. Engineering, National Taiwan University (2005) \\ B.S., National Taiwan University (2005) \\ Submitted to the Joint Program in Applied Ocean Science and Engineering \\ in partial fulfillment of the requirements for the degree of \\ Doctor of Philosophy \\ at the \\ MASSACHUSETTS INSTITUTE OF TECHNOLOGY \\ and the \\ WOODS HOLE OCEANOGRAPHIC INSTITUTION
}

February 2013

(C) Wu-Jung Lee, MMXIII. All rights reserved.

The author hereby grants to MIT and WHOI permission to reproduce and to distribute publicly paper and electronic copies of this thesis document in whole or in part in any medium now known or hereafter created.

Author

Joint Program in Applied Ocean Science and Engineering

January 11, 2013

Certified by

Timothy K. Stanton

Senior Scientist, Woods Hole Oceanographic Institution

Thesis Supervisor

Certified by

Andone C. Lavery

Associate Scientist, Woods Hole Oceanographic Institution

Thesis Supervisor

Certified by

Peter L. Tyack

Scientist Emeritus, Woods Hole Oceanographic Institution

Thesis Supervisor

Accepted by

David E. Hardt

Chairman, Department of Mechanical Engineering Committee on Graduate Theses Massachusetts Institute of Technology Accepted by

Henrik Schmidt

Chairman, Joint Committee for Applied Ocean Science and Engineering Massachusetts Institute of Technology/Woods Hole Oceanographic Institution 


\title{
Broadband and statistical characterization of echoes from random scatterers: application to acoustic scattering by marine organisms
}

\author{
by \\ Wu-Jung Lee
}

Submitted to the Joint Program in Applied Ocean Science and Engineering on January 11, 2013, in partial fulfillment of the

requirements for the degree of

Doctor of Philosophy

\begin{abstract}
The interpretation of echoes collected by active remote-sensing systems, such as sonar and radar, is often ambiguous due to the complexities in the scattering processes involving the scatterers, the environment, and the sensing system. This thesis addresses this challenge using a combination of laboratory and field experiments, theoretical modeling, and numerical simulations in the context of acoustic scattering by marine organisms. The unifying themes of the thesis are 1) quantitative characterization of the spectral, temporal, and statistical features derived from echoes collected using both broadband and narrowband signals, and 2) the interpretation of echoes by establishing explicit links between echo features and the sources of scattering through physics principles. This physics-based approach is distinct from the subjective descriptions and empirical methods employed in most conventional fisheries acoustic studies. The first part focuses on understanding the dominant backscattering mechanisms of live squid as a function of orientation. The study provides the first broadband backscattering laboratory data set from live squid at all angles of orientation, and conclusively confirms the fluidlike, weakly-scattering material properties of squid through a series of detailed comparisons between data and predictions given by models derived based on the distorted-wave Born approximation. In the second part, an exact analytical narrowband model and a numerical broadband model are developed based on physics principles to describe the probability density function of the amplitudes of echo envelopes (echo pdf) of arbitrary aggregations of scatterers. The narrowband
\end{abstract}


echo pdf model significantly outperforms the conventional mixture models in analyzing simulated mixed assemblages. When applied to analyze fish echoes collected in the ocean, the numerical density of fish estimated using the broadband echo pdf model is comparable to the density estimated using echo integration methods. These results demonstrate the power of the physics-based approach and give a first-order assessment of the performance of echo statistics methods in echo interpretation. The new data, models, and approaches provided here are important for advancing the field of active acoustic observation of the ocean.

Thesis Supervisor: Timothy K. Stanton

Title: Senior Scientist, Woods Hole Oceanographic Institution

Thesis Supervisor: Andone C. Lavery

Title: Associate Scientist, Woods Hole Oceanographic Institution

Thesis Supervisor: Peter L. Tyack

Title: Scientist Emeritus, Woods Hole Oceanographic Institution 


\section{Acknowledgments}

Many people have helped me and brought changes to all aspects of my life in these five and half years, and I thank you all.

I especially would like to thank my three advisors, Dr. Timothy Stanton, Dr. Andone Lavery, and Dr. Peter Tyack. In addition to all the great advice and words of wisdom I have received from them, I thank them for their full support for my (often unfocused) curiosity in acoustics and marine mammal echolocation and behavior, and each of their own ways to keep my on track so that I actually accomplished something along this constant wander. I have learned so much from their enthusiasm for science and relentless rigor in research. It has truly been a great experience and pleasure working with them. I also want to thank my committee member, Dr. James Preisig, for his encouragement in those frustrating moments of research and his enlightening words that propel me to pursue my interest in animal echolocation after graduate school. I also want to thank my committee member, Prof. John Leonard, for his helpful suggestions and support throughout the process and especially during the dark days of thesis-writing.

I would like to thank the following agencies for their financial support during my study: Taiwan Merit Scholarship (NSC-095-SAF-I-564-021-TMS), Office of Naval Research (ONR; grants N00014-10-1-0127, N00014-08-1-1162, N00014-07-1-1034), National Science Foundation (NSF; grant OCE-0928801), Naval Oceanographic Office (grant N62306007-D9002), WHOI Ocean Life Institute, and the WHOI Academic Programs Office funds.

I thank everyone in the WHOI Ocean Acoustics and Signals Lab for their always thought-provoking questions and enjoyable conversations regarding issues both related to and outside of acoustics and signals. I thank everyone in the WHOI Marine Mammal group for the wonderful and unorganized chats that happened spontaneously whenever I had a chance to walk into the Marine Research Facility. I thank Dr. Michael Jech in the NOAA Northeast Fisheries Science Center for his valuable suggestions from squid tethering to fish echo analysis. I also want to thank Dr. Roger 
Hanlon and everyone in the MBL Cephalopods Lab, who helped me enormously in handling and anesthetizing squid for the backscattering experiment. I also thank the WHOI Academic Programs Office and the MIT Mechanical Engineering Graduate Office for their administrative help.

As someone who can't seem to separate work and life very well, I am especially grateful to the following colleagues/friends for too many reasons than there is space for: Kyungmin Baik, Saurav Bhatia, Alessandro Bocconcelli, Tim Duda, Jim Doutt, Jonathan Fincke, Ken Foote, Ankita Jain, Frants Jensen, Ben Jones, Gareth Lawson, Ying-Tsong Lin, Raymond Lum, Jim Lynch, Aran Mooney, Arthur Newhall, Ananya Sen Gupta, Laela Sayigh, Cindy Sellers, Ilya Udovydchenkov, Gordon Zhang. I also want to thank my friends from MIT, WHOI, and elsewhere in the world that have not been mentioned above: Heather Beem, Ruo-Dong Chen, S. Carol Huang, Tzu-Hsin Huang, Kuo-Fang Huang, Ai-Chun Lee, Erin LaBrecque, Nien-Yun Liu, Marilena Oltmanns, Alexis Rudd, Jordan Stanway, Liching Wang, Chiou-Ju Yao, Hsin-Yi Yu, Derya Akkaynak Yellin. I really enjoyed the time we spent together in the woods, at sea, and in other random places, and I thank you for being there for me when I needed the most. Finally, I would like to thank my family, especially my parents, for their unconditional love that has always made me feel safe to be brave. 


\section{Contents}

1 Introduction $\quad 27$

1.1 Motivation . . . . . . . . . . . . . . . . . . 27

1.2 Acoustic scattering from individual marine organisms . . . . . . . . 29

1.2.1 Principles of acoustic scattering . . . . . . . . . . . . . . . 29

1.2.2 Scattering models . . . . . . . . . . . . . . . . . . 30

1.3 Measurement and analysis of $i n$-situ echo . . . . . . . . . . . . . . . . 34

1.3.1 Echo measurements in field experiments . . . . . . . . . . . . 34

1.3.2 Echoes from individual marine organisms . . . . . . . . . . . 36

1.3.3 Echoes from an aggregation of marine organisms . . . . . . . 38

1.3.4 Analysis of narrowband echoes from aggregations of marine organisms ....................... 40

1.4 Application of broadband signals in acoustic scattering studies . . . . 42

1.5 Statistical analysis of echo fluctuations . . . . . . . . . . . . 44

1.6 Thesis overview and specific topics . . . . . . . . . . . . . . . 48

2 Orientation dependence of broadband acoustic backscattering from squid $\quad 53$

2.1 Introduction . . . . . . . . . . . . . . . . . . 53

2.2 Experimental methods . . . . . . . . . . . . . . 58

2.2.1 Squid used in the experiment . . . . . . . . . . . . . . 58

2.2.2 Tank and instrument setup ............. 59

2.2.3 Experimental procedure ............... 60

2.2.4 Acoustic signal analysis and calibration . . . . . . . . . . 61 
2.2.5 Subtraction of background reverberation and control of data quality . . . . . . . . . . . . . . . . 65

2.3 Acoustic backscattering theory and modeling . . . . . . . . 66

2.3.1 Basic definitions of acoustic backscattering quantities . . . . 66

2.3.2 Distorted-wave Born approximation for acoustic backscattering: Application to squid . . . . . . . . . . . . . 66

2.3 .3 Model predictions . . . . . . . . . . . . . . . . . . 70

2.4 Data-model comparison . . . . . . . . . . . . . . . . 78

2.4.1 Time domain compressed pulse output (CPO) characteristics . 78

2.4.2 Angular variation of target strength (TS) at fixed frequencies . 80

2.4.3 Frequency dependence of TS at near normal incidence . . . . . 84

2.4.4 TS averaged over angle-of-orientation distribution . . . . . . 87

2.5 Statistics of echoes from individual squid . . . . . . . . . . . 91

2.5.1 Statistics of echoes from near or at normal incidence . . . . 92

2.5.2 Statistics of echoes from all angles of orientation . . . . . . 103

2.6 Discussion . . . . . . . . . . . . . . . . . . . . . . . . 106

2.6.1 Model performance . . . . . . . . . . . . . . . 106

2.6.2 Squid tissue material properties . . . . . . . . . . . 107

2.6.3 Scattering contribution from other potential sources . . . . . 107

2.6.4 Squid size estimation . . . . . . . . . . . . . . . . 108

2.6 .5 Squid shape . . . . . . . . . . . . . . . . . . . . 109

2.6.6 Modeling squid aggregations . . . . . . . . . . . . 110

2.6.7 Statistics of echoes from individual squid . . . . . . . . . 111

2.7 Summary and conclusions $\ldots \ldots \ldots \ldots \ldots \ldots \ldots$

3 Statistics of echoes from mixed assemblages of scatterers with different scattering amplitudes and numerical densities 115

3.1 Introduction . . . . . . . . . . . . . . . . 115

3.2 Theoretical development of characteristic function (CF)-based mixed assemblage $\mathrm{pdf} \ldots \ldots \ldots \ldots \ldots \ldots \ldots \ldots$ 
3.2 .1 Problem setup . . . . . . . . . . . . . . . . . . . . . . 121

3.2.2 Method of characteristic functions - beampattern effects not explicit . . . . . . . . . . . . . . . . . . 122

3.2.3 Incorporating beampattern effects . . . . . . . . . . . . . . . 124

3.2.4 Echoes from mixed assemblages . . . . . . . . . . . . 125

3.3 Numerical validation and examples of CF-based mixed assemblage pdfs 126

3.3.1 Simulated data generation . . . . . . . . . . . . . . 127

3.3.2 CF-based mixed assemblage pdf generation . . . . . . . . . 128

3.3.3 Validation of CF-based mixed assemblage pdf (as a predictor) 128

3.3.4 Effect of mixed assemblage composition on the echo pdf . . . . 131

3.4 Comparison of the CF-based mixed assemblage pdf and $M$-component mixture model . . . . . . . . . . . . . . . . . . . . . . . . . . . . . 132

3.4.1 Echo statistic models formulated for two types of scatterers . . 133

3.4.2 Method for inferring parameters of mixed assemblage . . . . . 134

3.4.3 Performance of models as inference tools . . . . . . . . . 135

3.5 Summary and conclusion . . . . . . . . . . . . . . . . . . . . . . 142

4 Statistics of broadband echoes: application to estimating numerical density of fish 145

4.1 Introduction . . . . . . . . . . . . . . . . . . . . . . . . 145

4.2 Numerical simulation of broadband echo pdfs . . . . . . . . . 150

4.2.1 Modeling framework for numerical simulation of broadband echo pdf . . . . . . . . . . . . . . . . 150

4.2.2 Effects of broadband beampattern response . . . . . . . . 153

4.2 .3 Effects of signal characteristics . . . . . . . . . . . . 157

4.2.4 Scatterer response . . . . . . . . . . . . . . . . . 159

4.2.5 Broadband echo pdfs from monotype aggregations and mixed assemblages . . . . . . . . . . . . . . . . . 162

4.3 Broadband acoustic backscattering data from fish aggregations in the ocean . . . . . . . . . . . . . . . . . . . . . 163 
4.3.1 Data collection and system calibration . . . . . . . . . 163

4.3.2 Echo data selection . . . . . . . . . . . . . . . 165

4.3.3 Echo pdf from fish aggregations . . . . . . . . . . . . 168

4.4 Estimation of the numerical density of fish in monospecific aggregations 172

4.4.1 Numerical density estimation using broadband echo pdf models 172

4.4.2 Numerical density estimation using measured $\mathrm{S}_{\mathrm{V}}$ and modeled $\mathrm{TS} \ldots \ldots \ldots \ldots \ldots \ldots \ldots$

4.4.3 Comparison of the estimated numerical density of fish . . . . . 181

4.4.4 Errors associated with the estimation of the numerical density of fish . . . . . . . . . . . . . . . . . 182

4.5 Summary and Conclusion . . . . . . . . . . . . . . 186

5 Summary of contributions and recommendations for future research $\begin{array}{ll}\text { directions } & 189\end{array}$

5.1 Contributions and significance . . . . . . . . . . . . . . 190

5.1.1 Broadband backscattering from live squid . . . . . . . . . . 190

5.1.2 Statistics of echoes from arbitrary aggregations of scatterers . 191

5.1.3 Summary of contributions of thesis work . . . . . . . . . . . 194

5.2 Recommendations for future research directions . . . . . . . . . . 195

5.2.1 Backscattering from squid . . . . . . . . . . . 195

5.2.2 Statistics of echoes from aggregations of scatterers . . . . . . 198

5.2.3 General approaches for the interpretation of echoes . . . . . . 201

5.3 Broader impacts . . . . . . . . . . . . . . . . 204

$\begin{array}{ll}\text { A Normalization of echo pdfs } & 207\end{array}$

B Addition of noise to echo pdf models 209 


\section{List of Figures}

1-1 Conceptual diagram of the problems studied in this thesis and the approaches taken for the analysis of the echoes. . . . . . . . .

2-1 (a) The pulse-echo system and experimental setup for the laboratory measurements of scattering from squid as a function of angle of orientation. The shaded box represents the NI (National Instruments, Inc.) system containing the central LabVIEW control program. (b) Tethering system used in the experiment and the definition of angle of orientation relative to incident acoustic signal. Solid lines represent monofilament lines outside of the squid body. Dashed lines represent monofilament lines running through the mantle cavity. . . . . . . . .

2-2 (a) Transmit signal measured at the output of the power amplifier. (b) Received calibration signal. (c) Spectrum of the received calibration signal. (d) Envelope of the autocorrelation function of the received calibration signal, normalized to the peak maximum at $0 \mu \mathrm{s} . \ldots$.

2-3 Various squid shapes used in this study: (a) the arms-splayed and (b) arms-folded squid shape without modification of the fins or randomization of the arms. (c-e) examples of hybrid randomized squid shapes with three different shapes of the fins (see Sec. 2.3.3 for detail of the

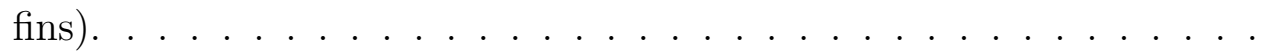


2-4 TS prediction versus angle of orientation at four frequencies $(60,70$, $85,100 \mathrm{kHz}$ ) for the three-dimensional DWBA numerical model using arms-folded squid shapes with and without the fins, and the analytical DWBA prolate spheroid model. The arrow indicates the scattering contribution from the fins. . . . . . . . . . . . .

2-5 TS predictions versus frequency for the three-dimensional DWBA numerical model using arms-folded squid shape and the analytical DWBA prolate spheroid model at four angles of orientation $\left(0^{\circ}, 45^{\circ}, 90^{\circ}, 135^{\circ}\right.$ from normal incidence). The usable band (gray area) in the experiment lies entirely in the geometric scattering region. . . . . . . . . .

2-6 Compressed pulse output envelope of (a) the analytical DWBA prolate spheroid model, and the three-dimensional DWBA numerical model using two fixed squid shapes through two full rotations $\left(720^{\circ}\right)$ : (b) the arms-folded configuration, and (c) the arms-splayed configuration. The compressed pulse output envelopes are normalized to the maximum envelope value in each of the plots. The symmetric sinusoidal pattern in (a) corresponds to the front and back interface of the prolate spheroid with respect to the incidence field, and the strong sinusoidal pattern in (b) and (c) corresponds to the location of the squid arms during the rotation. . . . . . . . . . . . . . . .

2-7 Noise addition procedure for model predictions. (a) The frequency dependent background noise profile (including reverberation) across the usable band of the experiment. (b) TS predictions with noise added (top row) and without noise added (bottom row) based on the threedimensional DWBA numerical model. The solid line is the mean of the measured or added noise. The gray or white area between the two dashed lines indicates the range between \pm 1 standard deviation from the mean. The brackets indicate regions where the effect of noise addition is more prominent. Model predictions below the noise threshold were omitted. . . . . . . . . . . . . . . . . 
2-8 Temporal characteristics of the scattering at normal incidence. (a) Model predictions given by the three-dimensional DWBA numerical model with arms-folded and arms-splayed squid shapes and the analytical DWBA prolate spheroid model. (b-d) Experimental data from three individuals, each with 15 individual pings overlaid at normal incidence. All compressed pulse output envelopes (model prediction and data) were normalized to the maximum value in each model prediction or each ping. . . . . . . . . . . . . . . . . .

2-9 Compressed pulse output envelope of (a) the experimental data from three individuals and (b) the three-dimensional DWBA numerical model using a hybrid squid shape with randomized arms over two full rotations $\left(720^{\circ}\right)$. The compressed pulse output envelopes are normalized to the maximum envelope value in each of the plot. Faint vertical lines in the experimental data are due to noise not effectively eliminated by the background reverberation subtraction. . . . . . . . . . .

2-10 Data-model comparison of TS versus angle of orientation at four frequencies $(60,70,85,100 \mathrm{kHz})$ for the individual 0822a. Hybrid randomized squid shapes with three fin shapes were used in the threedimensional DWBA numerical model: (a) original asymmetric fins, (b) artificial symmetric fins, (c) no fins. The experimental data are represented by dots. The gray area indicates the range of \pm 1 standard deviation from the mean of the model predictions. The arrow indicates the scattering contribution of the fins. The cut-off pattern near the bottom of each plot is resulted from omitting experimental data and model predictions lower than the noise threshold. . . . . . . . 
2-11 Data-model comparison of TS versus angle of orientation at four frequencies $(60,70,85,100 \mathrm{kHz})$ for three representative individuals (0822a, 0814a, 0819c). The hybrid randomized squid shapes with no fins were used in the three-dimensional DWBA numerical model, with the size of the squid shape scaled to match that of each individual. The arrows indicate the potential deviation of angles of orientation during the experiment. Other details on the figure is given in the caption of

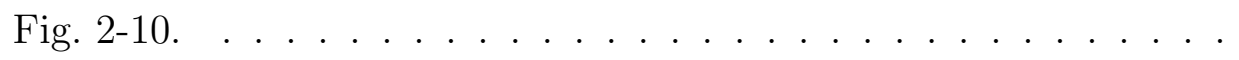

2-12 Comparison of the performance of the three-dimensional DWBA numerical model and the analytical DWBA prolate spheroid model at two frequencies Frequency-dependent noise was added to both models to enable valid comparison with the data. Dots represent the pingby-ping experimental data for the individual 0822a. The gray area indicates the range of \pm 1 standard deviation from the mean of the models. Note that the experimental data and model predictions lower than the background noise threshold (black lines) were not omitted in here to illustrate the difference clearly. . . . . . . . . . . . .

2-13 Data-model comparison of TS spectra averaged between $\pm 2^{\circ}$. The angle of orientation of experimental data were adjusted by $\theta_{a d j}$ in cases where potential deviation in the angles of orientation were observed (see Fig. 2-11). . . . . . . . . . . . . . . . . . . .

2-14 Averaged TS versus frequency for the experimental data, the analytical DWBA prolate spheroid model, and the three-dimensional DWBA numerical model using both fixed and hybrid randomized squid shapes in two planes (data only available in the lateral plane). All averages were done in the linear domain over \pm 2 standard deviations $(\sigma)$ from the mean angle $(\mu)$ and converted to TS. (a) Averages in the dorsal-ventral plane. (b) Averages in the lateral plane. . . . . . . . . . . . . . . 
2-15 Averaged TS versus frequency for the experimental data, the analytical DWBA prolate spheroid model, and the three-dimensional DWBA numerical model using both fixed and hybrid randomized squid shapes in the lateral plane. Results from three individual squid are shown. Only a subset of models from those used in Fig. 2-14 are plotted in this figure for clarity and to facilitate inter-individual comparison. . .

2-16 Echo pdf of data collected within the range $\left[75^{\circ}, 105^{\circ}\right]$ (within $\pm 15^{\circ}$ from normal incidence). The background noise thresholds are plotted for reference since all data were pooled to avoid problems in the normalization of echo pdf (see text). The figures in the left-most column contain identical information as those in the second column (data from individual 0822a) and are included to facilitate the comparison between results plotted in linear-linear and log-log axes. . . . . . . . .

2-17 Compressed pulse output envelopes at normal incidence for individuals 0822a and 0814a. The raw compressed output envelopes on the left show the temporal variation of the magnitude of the scattering response across pings, and the normalized compressed pulse output envelopes on the right show more detail of the temporal characteristics for each ping. The compressed pulse output envelopes on the right are normalized to the maximum value in the envelope time series of each ping. . . . . .

2-18 TS spectra averaged over all pings shown in Fig. 2-17. The same set of models as those used in Fig. 2-13 are shown here for reference. . . 96

2-19 Echo pdf of data collected at normal incidence only for individuals 0822a and 0814a. Arrows indicate the narrowly-distributed echo amplitude near the mode. Details on the figures are given in the caption to Fig. 2-16. Note that noise thresholds are not plotted in some cases when all echoes are above the threshold. . . . . . . . . . . . . . 97

2-20 Echo pdf of data collected within the range $\left[60^{\circ}, 120^{\circ}\right]$ (within $\pm 30^{\circ}$ from normal incidence). Details on the figures are given in the caption to Fig. 2-16. . . . . . . . . . . . . . . . . . 
2-21 Echo pdf of data collected within the range $\left[30^{\circ}, 150^{\circ}\right]$ (within $\pm 60^{\circ}$ from normal incidence). Details on the figures are given in the caption to Fig. $2-16 . \ldots \ldots \ldots$

2-22 Comparison of echo pdfs of data collected within the ranges $\left[75^{\circ}, 105^{\circ}\right]$, $\left[60^{\circ}, 120^{\circ}\right],\left[30^{\circ}, 150^{\circ}\right]$, and $\left[0^{\circ}, 360^{\circ}\right]$. Details on the figures are given in the caption to Fig. 2-16. . . . . . . . . . . . . . . . . . . . 101

2-23 Echo pdf of data collected within the range $\left[0^{\circ}, 60^{\circ}\right) \bigcup\left(120^{\circ}, 180^{\circ}\right]$. These echoes form a complementary set of data to those shown in Fig. 2-20. Details on the figures are given in the caption to Fig. 2-16. 102 2-24 Echo pdf of data collected measured at all angles of orientation $\left(\left[0^{\circ}, 360^{\circ}\right]\right)$. The best-fitting randomly-rough prolate spheroid models are plotted with corresponding aspect ratio $(\epsilon)$ indicated. The model and fitting procedure are described in Sec. 2.5.2. Details on the figures are given in the caption to Fig. 2-16. . . . . . . . . . . . . . . . . . . 104

3-1 Illustration of analysis windows containing two possible spatial arrangements for aggregations composed of more than one type of scatterer. (a) Scatterers of the same type are separated into their own subregion. (b) Scatterers of different types are uniformly interspersed throughout the analysis window. In each case, the sonar/radar resolution cell is much smaller than the analysis window and, in case (a), is also much smaller than each subregion. . . . . . . . . . . . . . . . 119

3-2 Validation of the theoretical CF-based mixed assemblage pdf using (3.10) and (3.13) (lines) with numerically-simulated echo pdfs (symbols) for the case of two types of scatterers uniformly interspersed as in Fig. 3-1(b). The number of weak scatterers, $N_{w}$, is fixed at 100 whereas the number of strong scatterers, $N_{s}$, and the ratio of the magnitudes of scattering amplitudes of the strong to the weak scatterers, $r_{s w}$, are both varied. . . . . . . . . . . . . . . . 129 
3-3 Comparison of echo pdfs from monotype aggregation and mixed assemblages with varying number of scatterers and $r_{s w}$. The number of dominant scatterers, $N_{d o m}$, is indicated for each plot. $N_{d o m}$ equals the total number of scatterers in monotype aggregations the number of strong scatterers in mixed assemblages (i.e., $N_{d o m}=N_{s}$ ). $N_{w}$ is fixed at 100 for all mixed assemblages. . . . . . . . . . . . . . . . 130

3-4 Variation of the CF-based mixed assemblage pdf as a function of mixed assemblage composition. The top plots show the effect of changing $N_{s}$ $(=1,5,10,20,50,100)$ on the shape of the echo pdf when $r_{s w}=5$ and $N_{w}=100$. The bottom plots show the echo pdf variation with the same combinations of $N_{s}$ and $N_{w}$, but with $r_{s w}=20 . \ldots . .$.

3-5 Several representative examples of the echo pdf of simulated mixed assemblages $(\bigcirc)$ and the corresponding best-fitting model pdfs (lines). Also shown on the plots are the true values of $r_{s w}$ and $N_{s}$ used to generate the simulated data. The best-fitting assemblage composition parameters for the models are summarized in Fig. 3-6. The arrows indicate the locations where the best-fitting mixture models have noticeable divergence from the data. . . . . . . . . . . . . . . . 136

3-6 Comparison of the best-fitting assemblage composition parameters versus the true parameters for the CF-based mixed assemblage pdf $(\triangle)$, the 2-component CF-based mixture model $(\bigcirc)$, and the 2-component Rayleigh mixture model $(\bullet)$. The squares $(\square)$ and crosses $(\times)$ indicate the cases when the best-fitting models are composed of only one CFbased pdf component. The vertical dashed lines show the approximate locations where the performance of the CF-based mixed assemblage pdf as an inference tool starts to degrade. Note that the 2-component Rayleigh mixture model does not contain parameters $N_{w}$ and $N_{s}$. . 137

3-7 Comparison of CF-based mixed assemblage pdf produced with varying

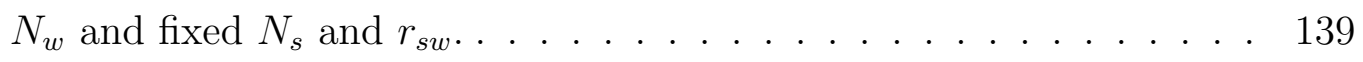


3-8 Comparison of CF-based mixed assemblage pdf produced with varying $r_{s w}$ (as shown on the legend) and fixed $N_{s} . N_{w}$ is fixed at 100 for all cases. . . . . . . . . . . . . . . . . . . . . . . 140

4-1 A schematic of one realization of the numerical model showing important elements in the modeling framework. The model uses broadband signals and the echoes are processed using pulse compression before the envelopes are taken. The sample point is arbitrarily selected in the region away from the edges of the range gate, $\overline{\mathrm{AB}}\left(=L_{g}\right.$ in text). A transducer with a circular aperture is used here for illustration, and can be substituted according to the specific system parameter. A low-frequency and a high-frequency narrowband beampattern (BP) are sketched to illustrate the frequency-dependent property of the beampattern. Note that the echoes are modulated across all frequencies according to their locations in the beam. Compared to the other two scatterers, scatterer \#2 is located within the mainlobe of the beam and therefore results in a sharper echo (see Sec. 4.2.2). . . . . . . . . . . . 151

4-2 Block diagram of the numerical simulation procedure. All involving components are discussed in Sec. 4.2. Note the block diagram depicts the procedure to generate one realization. The ensemble of samples collected from multiple realizations is used for the estimation of the echo pdf model. . . . . . . . . . . . . . . . . . . . . . . . 154 
4-3 (a) Narrowband beampatterns with respect to the polar angle $(\theta)$ of a circular aperture at three frequencies $(30,50$, and $70 \mathrm{kHz})$. The aperture has a radius of $0.054 \mathrm{~m}$, chosen to match the specification of the high-frequency broadband echosounder used in the field experiment (AirMarLow channel, Sec. 4.3.1). (b) Two-way beampattern pdf $\left[\mathrm{P}_{\mathrm{B}}(b)\right.$, thin black line $]$ and the associated echo pdf produced with only one scatterer in the resolution cell $\left[\mathrm{P}_{\mathrm{A}}(a)\right.$, thick black line]. Definition of these quantities can be found in Sec. 3.2.3. Unless otherwise specified, the parameters of the transducer given here are used for all modeling results presented in this chapter. . . . . . . . . . . . . . . 155

4-4 (a) The impulse responses of the two-way beampattern at three different polar angles $\left(\theta=5^{\circ}, 10^{\circ}\right.$, and $\left.30^{\circ}\right)$. (b) Time domain characteristics of the autocorrelation functions of different signals modified by the beampattern impulse response. The widths of the responses are jointly determined by the bandwidths and frequency contents of the signals. . . . . . . . . . . . . . . . . . . . . . . 156

4-5 Broadband echo pdf models generated with and without the beampattern effects in three cases with different number of scatterers $(N)$ in the range gate. This comparison shows the strong non-Rayleigh influence of the beampattern effects. The Rayleigh distribution is plotted as a background reference for all echo pdf figures in this chapter. Unless otherwise specified, all echo pdfs in this study are normalized according to the procedure in Appendix A. . . . . . . . . . . . . . 156 
4-6 Spectra (a), autocorrelation functions (b), and echo pdfs (c) generated using different signals in cases with different number of scatterers in the range gate $(N)$. Comparisons are made between: two linear chirp signals shaded using a narrow Hann window (Narrow Hann) and a wide Hann window (wide Hann); two linear chirp signals shaded using a narrow Hann window (Narrow Hann) and a wide Hann window (wide Hann); and the ideal (Ideal Tx) and actual (Actual Tx) transmit signal associated with the high-frequency broadband echosounder used in the field experiment. . . . . . . . . . . . . . . . . . . . .

4-7 Comparison of echo pdf models generated using scatterers with amplitude distributions following the Rayleigh distribution and the distribution of a randomly-rough prolate spheroid (aspect ratio $\xi=5$ ) randomly-oriented in a three-dimensional space. The influence of the strong non-Rayleigh characteristics of the scattering from the rough prolate spheroid is evident in the tail of the resultant echo pdf. . . . .

4-8 Comparison of echo pdf models for monotype aggregation and twocomponent mixed assemblages with varying composition. The ratio between the backscattering cross section of the strong to the weak scatterers in the mixed assemblage $\left(r_{\mathrm{sw}}\right)$ is varied from 5 to 30 . The number of dominant scatterers in the range gate $\left(N_{\text {dom }}\right)$ is defined to be equal to the number of scatterers in monotype aggregations and the number of strong scatterers in mixed assemblage, i.e., $N_{\text {dom }}=$

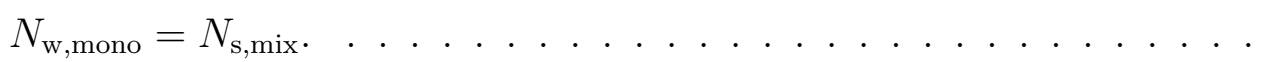

4-9 Distribution of the length of herring concluded from trawl net catches [reproduced from Fig. 4(c) of Stanton et al. (2012)] . . . . . . . . . . 165 
4-10 Calibration data and results for the three broadband channels (Shamu: $1-6 \mathrm{kHz}, 424: 10-18 \mathrm{kHz}$, and AirMarLow: $30-70 \mathrm{kHz}$ ) channels in the EdgeTech broadband echosounder. (a) Sphere echoes selected for use in the calibration. Each echo is plotted as a circle $(\bigcirc)$ according to the roll and pitch angles of the towbody recorded at the instant of sonar transmission. The circles are color-coded according to the relative amplitudes of the peaks of the echo envelopes, with the red-to-blue variation denotes high-to-low amplitude variation. Circles marked with '*' are echoes with the top 5\% highest amplitudes. Echoes within an arbitrarily-chosen $1.5^{\circ}$ radius from the mean roll and pitch angles of these marked echoes (further marked by larger red circles) are selected for use in the calibration. This procedure is employed to exclude echoes resulted from off-axis insonification of the standard sphere. (b) Time-series of the envelopes of the selected echoes. Echoes from the 424 and AirMarLow channel are adjusted so that the peaks of the envelopes of specular reflections are aligned to facilitate paritialwave analysis. Echoes from the Shamu channel are not adjusted. (c) Results of calibration for all three channels. Results for both the fullwave and partial-wave analyses are shown for the 424 and AirMarLow channel. . . . . . . . . . . . . . . . . 166

4-11 Examples of data and analysis of monotype (monospecific) aggregations of herring observed near the seafloor in the day time. (a) Echogram of the aggregation with two analysis windows. (b) Broadband volume backscattering strengths $\left(\mathrm{S}_{\mathrm{V}}\right)$ and their respective best-fitting broadband physics-based hybrid models for the two analysis windows marked in (a) (Sec. 4.4.2). Also shown are the ranges of frequency $(36.3-39.7$ $\mathrm{kHz}$ ) included for the analysis of narrowband $S_{V}$. (c) Broadband echo pdfs of the data and best-fitting models(Sec. 4.4.1) . . . . . . . . . . 169 
4-12 Examples of data and analysis of mixed assemblages of fish observed near the sea surface at night. (a) Echogram of the mixed assemblage with two analysis windows. (b) Broadband volume backscattering strengths $\left(\mathrm{S}_{\mathrm{V}}\right)$ for the two analysis windows marked in (a). (c) Broadband echo pdfs of the data. Note the prominent highly-elevated tails that may have been caused by occasional insonification of large fish in the mixed assemblage. This aggregation is the same as aggregation E that has been analyzed in Stanton et al. (2012). . . . . . . . . . . . . 170

4-13 Examples of monotype broadband echo pdf model used in the inference analysis. The shape of the echo pdf varies from highly non-Rayleigh toward the Rayleigh distribution with increasing number of scatterers in the range gate $(N)$. The echo pdf model's shown here are produced with $N=10,50,100,200,500$, and 800. Rayleigh-distributed noise was added to each model following the procedure described in Appendix B. 173

4-14 (a) Two model shapes for the fish swimbladder. The first model uses the shape of a prolate spheroid. The second model uses the crosssectional profile of a prolate spheroid, but with its center adjusted to achieve a flat dorsal surface. The specific shapes shown here are generated assuming a swimbladder volume of $13 \mathrm{~mL}$ at sea surface for a $25 \mathrm{~cm}$ herring. (b) Examples of predicted averaged TS of this fish at a depth of $170 \mathrm{~m}$ evaluated using different angle of orientation distributions. The distributions of angle of orientation used here are normal distributions with different mean values $\left(\theta_{\text {mean }}=0^{\circ}, 5^{\circ}\right.$, and $\left.10^{\circ}\right)$ and an identical standard deviation $\left(\theta_{\text {std }}=3^{\circ}\right)$. . . . . . . . . . 178 
4-15 Comparison of TS predictions of swimbladder resonance given by two models. The first model (Ye) was derived assuming a prolate spheroidal shape (Ye, 1997), whereas the second model (Love) assumes a spherical shape with equivalent volume (Love, 1978). The low-frequency TS predictions are plotted along with the high-frequency TS predictions given by the modal-series-based deformed cylinder model (HF) using a swimbladder shape with flat dorsal surface. The calculation uses the same parameters as in Fig. 4-14. . . . . . . . . . . . . . . . .

4-16 Comparison of TS predictions in the swimbladder resonance region with different values of viscosity for fish flesh $(\xi)$ using Ye's model (Ye, 1997). Here, the value of $\xi$ varies from 10 to $60 \mathrm{~Pa} \cdot \mathrm{s}$ at an interval of $10 \mathrm{~Pa} \cdot \mathrm{s}$. Calculations were made using the same parameters as in Fig. 4-14 and Fig. 4-15. . . . . . . . . . . . . . . . . . . . . 180

4-17 (a) Echogram of the monospecific herring aggregation with analysis windows. Windows \#1 and \#2 are identical to the two windows shown in Fig. 4-11. (b) Numerical density of fish estimated using various methods. Results are plotted along with their respective confidence intervals. . . . . . . . . . . . . . . . . . . . 182

A-1 Comparison of several un-normalized (top) and normalized (bottom) echo pdfs calculated using the CF-based echo pdf formula with varying numbers of only one type of scatterer $(N)$. . . . . . . . . . . 208

B-1 (a) Comparison of the pdfs of squid echoes and background noise, as well as their corresponding Rayleigh pdfs (see text). (c) Comparison of rough prolate spheroid echo pdf model's generated with and without added noise. . . . . . . . . . . . . . . . . 
B-2 (a) Echogram of the fish aggregation with the analysis windows for fish echoes and background noise. Note that the depth of the echosounder is not corrected in this echogram so that the bottom appears to undulate as opposed to the flat bottom shown in Fig. 4-11. (b) Comparison of the pdfs of fish echoes and background noise, as well as their corresponding Rayleigh pdfs (see text). (c) Comparison of broadband echo pdf model's generated with and without added noise. . . . . . . . . 212 


\section{List of Tables}

2.1 Dimensions and ranges of angle of orientation for the squid used in the acoustic backscattering measurements. All dimensional measurements were conducted when the animal was dead after the acoustic experiment was completed. The Total Length is the length from the tip of the mantle to the tip of the arms when the squid is placed flat on a surface. The Mantle Width is the width of the widest portion of the mantle on the dorsal side. The Mantle Length is the length between the two ends of the mantle on the dorsal side. Two numbers in the Range of Angle of Orientation Measured indicate that acoustic measurements were conducted twice on the same individual. The Calculated Weight was calculated based on the published length-weight relationship for L. pealeii (Lange and Johnson, 1981). . . . . . . . . . . . . . . . . . . 58

4.1 Summary of model parameters used in the broadband physics-based hybrid model. Here, the ratio of specific heat, $\gamma_{0}$, is 1.4 , and $D$ is the depth of the fish in meters. . . . . . . . . . . . . . . . . . 177

4.2 Summary of potential sources of errors and corresponding impact on the estimation results associated with model assumptions used in this study. . . . . . . . . . . . . . . . . . . 184 


\section{Chapter 1}

\section{Introduction}

\subsection{Motivation}

Active remote-sensing systems acquire information by transmitting signals and receiving echoes from the subject(s) of interest. One major advantage of such systems over direct on-site measurements is the ability to provide synoptic data over a large spatial scale across temporal spans relevant to the goal(s) of the study. For research fields such as oceanography and atmospheric science, such information is often desirable so that comprehensive understanding of the interaction among various components involved in the system can be developed (Le Chevalier, 2002; Medwin and Clay, 1998). In essence, if direct measurements of a particular quantity is considered "point samples" from its distribution, remote-sensing systems provide information that "connects the dots" through the interpretation of echoes. Another primary advantage of remote-sensing systems is the ability to provide data from study sites that are difficult to access or under harsh conditions. For example, in oceanography, information is often needed from locations that are remote or at great depth, and the instruments are often damaged by wind, waves, currents, corrosion, bio-fouling, pressure, etc. To overcome these challenges, acoustic signals, which suffer significantly less attenuation in sea water than electromagnetic signals, have been widely used to probe and characterize both the ocean interior and its boundaries (Medwin and Clay, 1998; Urick, 1983). Through evolution, similar remote-sensing techniques have also 
been adopted by echolocating animals, such as bats and dolphins, for foraging and navigation (Au, 1993; Griffin, 1958).

The study of biological oceanography is vital in understanding marine ecosystems and carries social and economic consequences as human utilization of ocean resources for food and energy have increased steadily over the past few decades (Mann and Lazier, 2005; Roberts, 2002). Basic features of biological aggregations, including taxonomic composition, patchiness of their spatial distribution, and the transient nature of their occurrence, are of fundamental importance in constructing a complete picture of biological oceanography. Such information can be collected using a variety of techniques involving the use of nets, optics, and acoustics (Harris et al., 2000). Net-based methods have been traditionally used to allow precise identification of the organisms and collection of genetic samples as well as life history data including animal length, weight, growth of gonad, etc. However, these methods suffer from problems such as net-avoidance, damage to animals, and their inherent temporal and spatial sparsity. Optical methods are capable of providing information efficiently for animal identification and behavior observation, but are limited in range and the small sampling volume due to the strong attenuation of electromagnetic wave in sea water. Contrary to the above two methods that primarily deliver point samples with sparse temporal and spatial coverage, active acoustic methods, which can provide synoptic data in high resolution across relevant temporal and spatial scales, have therefore been used extensively as a complementary survey tool in biological oceanographic and fisheries studies (Klemas, 2012; Medwin and Clay, 1998).

However, the interpretation of acoustic echoes from marine organisms can be highly ambiguous, due to the complexity involved in the sound scattering processes. For example, the echoes are determined jointly by the properties of the transmitted signals, the propagation of sound to and from the scatterer(s), characteristics of the transmitting and receiving sensors (transducers), as well as the acoustic scattering features of the scatterer(s) (Medwin and Clay, 1998; Urick, 1983). Unique determination of the sources of scattering is often difficult, and requires detailed understanding of the influences of each of the above contributing components. This research is aimed 
at providing new data, models, and approaches that can serve as the basis of future development of accurate and reliable techniques for the interpretation of echoes. A brief review of the studies of acoustic scattering from marine organisms is given in the sections below, followed by an overview of the research conducted in this thesis.

\subsection{Acoustic scattering from individual marine or- ganisms}

\subsubsection{Principles of acoustic scattering}

The acoustic scattering properties of any given object can be fully described by its complex scattering amplitude, $f$, which has both spectral and directional dependencies determined by the target's shape, size, angle of orientation, and material properties, such as the mass density, $\rho$, and sound speed, $c$ (Medwin and Clay, 1998). In cases where the echoes are measured in the backscattering direction, as the dynamic range of the scattered signals is typically very large, a logarithmic measure of the backscattering amplitude is commonly used, defined as target strength (TS), expressed in units of decibels $(\mathrm{dB})$ relative to $1 \mathrm{~m}^{2}$, and given by $\mathrm{TS}=10 \log _{10}\left|f_{b s}\right|^{2}=10 \log _{10} \sigma_{b s}$, where

$\sigma_{b s}=\left|f_{b s}\right|^{2}$ is the differential backscattering cross section, and $f_{b s}$, or backscattering amplitude, is the scattering amplitude evaluated in the backscattering direction.

The scattering of a given object generally varies strongly as a function of frequency depending on the size of the object relative to the wavelength (Medwin and Clay, 1998). This functional dependency can be understood by comparing the acoustic wavelength $(\lambda)$ to the characteristic dimension of the object ( $a$, such as the radius for spherical object or the length of elongated objects) through the dimensionless quantities $k a$, where $k$ is the acoustic wavenumber, defined by $k=2 \pi / \lambda$. The $k a \ll 1$ case is usually referred to as the "Rayleigh scattering region", where the wavelength is much larger than the object and the backscattering cross section is generally proportional to $(k a)^{4}$ for objects without gas inclusion. When $k a \gg 1$, the acoustic waves can be approximated as "rays" in this "geometrical region", and the scattering occurs at the 
discontinuities within and at the boundaries of the object (Medwin and Clay, 1998).

In the intermediate region, however, the scattering characteristics are complex and contain prominent structures, such as resonances, depending on the detailed physical properties of the target (Ainslie and Leighton, 2011).

For objects with simple geometry and internal structure, such as spheres and spherical shells, the exact scattering functions can be derived analytically and used for benchmark assessment for the scattering from more complex objects or verification of other approximate solutions. The scattering functions can be obtained by solving the Helmholtz-Kirchhoff integral over the object boundary, or by expanding the solutions to the wave equations in convenient coordinate systems, and matching the boundary conditions at the interface of the scatterer and the medium. In the latter case, the scattering function can be understood as a series of "modes" supported by the particular geometry and material properties of the object. For example, the dominant resonance of a gaseous body in the $k a \ll 1$ region is trackable through the lowest mode in the modal-series solution, while the inclusion of higher modes gives a complete description for the scattering across all ranges of $k a$.

Although marine organisms are generally of much more complex outer shapes and internal composition, studies of the acoustic scattering properties of simple objects bring important insights that can be applied to understand the scattering from marine organisms, as will be seen in the next section.

\subsubsection{Scattering models}

Quantitative interpretation of echoes for biologically-relevant information requires detailed knowledge on the acoustic scattering characteristics of different types of marine organisms. Better understanding and modeling capabilities for the scattering from individual animals form the basis for accurate interpretation of echoes collected in the field (Medwin and Clay, 1998). Despite the often complex shape and internal structure of animal bodies, the acoustic scattering properties of marine organisms can generally be determined according to their gross anatomical features and material properties of important organs, with additional modification due to individual- 
or behavior-related characteristics. Such information is usually obtained using laboratory experiments with individual animal fixed on the main axis of the transducer and insonified at a designated set of angles of orientation in the far-fields of both the transducer(s) and the animal (Stanton, 2012). This setup allows direct observation of the scattering features from the animal, which are are useful for the development and verification of scattering models of individual animals. The following sections discuss the modeling of the acoustic scattering of three representative groups of marine organisms categorized according to their gross anatomical features: fluidlike, elastic-shelled, and gas-bearing (Stanton et al., 1998a,b). These three categories were established from studies on the scattering from fish and zooplankton, and can be extended to include other animals with similar anatomical features.

The fluidlike animals are represented by euphausiids, copepods, and decapod shrimps, whose bodies are composed primarily of weakly-scattering materials with sound speed and density very close to those of the sea water. The boundary of the animal behaves acoustically as a fluid-fluid interface and does not support shear waves. These properties prompt the use of a series of models incorporating the weaklyscattering material properties with increasingly complicated representation of the shape of the animal, including a modal-series-based line integral or a ray summation using a deformed cylinder formulation (Stanton, 1989; Stanton et al., 1993b), and a distorted-wave Born approximation (DWBA) formulation in which the scattering is evaluated by a volume integral (Chu et al., 1993; Morse and Ingard, 1987; Stanton et al., 1993a, 1998a). For fluidlike scatterers with elongated shapes, such as that of euphausiids, the scattering can be qualitatively described by two rays from the front and back interfaces between the animal body and the medium at normal incidence, and the scattering across all angles of orientation can be predicted using more sophisticated model that includes roughness and inhomogeneity within the body (Lavery et al., 2002; Stanton et al., 1998a). These dependencies have been verified by various experiments conducted both in the laboratory and in the field (Lawson et al., 2006; Stanton et al., 1998b).

The elastic-shelled animals, represented by gastropods in the ocean, are char- 
acterized by their possession of a hard elastic shell (Stanton et al., 1998a,b). The scattering is dominated by the dense elastic shell which gives rise to a series of highly complicated echoes from the specular reflection of the front interface, internal refraction and reflections within the the shell and the body, and a class of circumferential waves propagating on the shell and the immediate surrounding fluid (Stanton et al., 1998a). Although such decomposition of different scattering components can provide physical insights to the scattering mechanisms of these animals, the complexity of the boundary and shape of the elastic shell is challenging, and numerical evaluation of the wave equation or generalized ray theory is usually necessary for exact modeling of their scattering functions (e.g., Jansson, 1993; Kargl and Marston, 1989; Marston et al., 1990; Rebinsky and Norris, 1995).

The third category of animals is characterized by the inclusion of gas in their body (gas-bearing), such as fish with swimbladders and siphonophores possessing pneumatophores. Due to the large contrast between the air and seawater, the gas inclusion dominates the scattering from this type of animal, and features in the echoes can be explained by identifying the contributions from the gas bubble and the remaining fluidlike body or bony structure, if present (Foote, 1985; Reeder et al., 2004; Stanton et al., 1998a,b; Sun et al., 1985). Due the complexity involved, the scattering from gas-bearing animals is generally predicted using hybrid models consisting of separate models for different scattering regimes (Chu et al., 2006; Clay and Horne, 1994; Jech and Horne, 2002; Stanton et al., 2010).

For the scattering from the gas-bearing organ, when $k a<1$, the breathing mode dominates the scattering with an omnidirectional scattering pattern and a resonance behavior determined by the volume and associated damping parameters (reradiation, thermal, and viscous damping (Ainslie and Leighton, 2011; Medwin and Clay, 1998). Models are usually derived by solving for the scattering pressure from the wave equation using simple geometries, such as spheres or prolate spheroids (Feuillade and Nero, 1998; Love, 1978; Ye, 1997; Ye and Hoskinson, 1998), with appropriate boundary conditions and volumes equivalent to those of the gas-bearing organs. In the $k a>1$ region, on the other hand, the scattering becomes highly directional and 
is determined by the exact shape and orientation of the gas inclusion with respect to the incidence waves. The scattering in this region has been modeled by the coherent summation of a series of objects with simple geometries that jointly capture the shape of the gas-bearing organ. Examples include the Kirchhoff ray-mode model which approximates the swimbladder morphology by a series of finite-length cylinders (Clay and Horne, 1994; Jech and Horne, 2002), and the modal-series-based deformed cylinder model which evaluates the scattering through a line integral over circular slices with variable radius along an arbitrary center line (Stanton, 1989; Stanton et al., 2010). Another class of models often incorporates the complex outer shape of the swimbladder and uses sophisticated numerical methods such as the boundaryelement method to predict its scattering features (Foote and Francis, 2002; Francis and Foote, 2003). The scattering from the soft body is generally predicted using models consistent with the fluidlike material properties of the tissues, such the DWBA model, or modal-series-based deformed cylinder model and the Kirchhoff-ray model solved with fluidlike boundary conditions.

Although the scattering contributions from the gas-bearing organ and the body tissues add coherently to form the scattering from the whole animal, in practice these two components are usually summed incoherently to model the scattering from the whole animal. This operation is justified by the relatively weak contribution from the soft tissue and the smearing of the exact phase information due to the complicated morphology of the animals (Gorska and Ona, 2003b).

There remain unanswered questions in our understanding of the acoustic scattering from the above organisms. For example, as important as the swimbladder is in determining the scattering from fish, the depth-dependency of its geometry with respect to the behavior and life history stage of fish remains unclear (Diachok, 2005; Fässler et al., 2009a; Gorska and Ona, 2003b; Horne et al., 2009; Ona, 1990). This question is further complicated by the diversity in the anatomical features of fish swimbladders, among them the differences between physostomes (fish with a swimbladder connected to the stomach through a pneumatic duct) and physoclits (fish with a closed swimbladder) (as reviewed in Diachok, 2005). Furthermore, although 
key factors influencing the scattering of individual organisms, such as the distribution of the length, angle of orientation, depth, etc., have been identified in the models, the relative importance of each factor on the scattering, as well as the variability of these parameters needs to be quantified systematically in a biologically-meaningful context (Hazen and Horne, 2003; Lawson et al., 2006).

Although the models introduced above were derived based on the physical properties of zooplankton and fish, their application may be extended to predict the scattering from other animals with similar anatomical features and material properties. For example, the class of models developed for fluidlike zooplankton have been applied to predict the scattering from a variety of animals with fluidlike, weakly-scattering material properties, such as squid, fish without swimbladders, and other gelatinous zooplankton (Brierley et al., 2004; Jones et al., 2009; Kang et al., 2006; Warren and Smith, 2007; Wiebe et al., 2010; Yasuma et al., 2006, 2010). However, direct application of existing models should be treated with caution, since the scattering from any given animal may be strongly influenced by taxon-specific organs or structures. Therefore, it is important that dominant scattering mechanisms of different animals be identified through experiments, and detailed data-model comparison be conducted to assess the performance of existing models and guide further model development.

\subsection{Measurement and analysis of in-situ echo}

\subsubsection{Echo measurements in field experiments}

Active acoustic survey techniques infer biologically-relevant information through features in the scattered signals. Depending on the experimental scenario, acoustic scattering measurements can be categorized into three groups: monostatic, bistatic, and multistatic, according to the geometry among the transmitter, receiver, and scatterers (Medwin and Clay, 1998). The transmitters and receivers are spatially collocated for monostatic systems and are separated for the other two types of systems. Bistatic measurements refer to the scenarios in which there is one pair of spatially 
separated transmitter and receiver, while multistatic systems often involve multiple sets of spatially diverse monostatic or bistatic sensors. Most acoustic scattering studies of marine organisms are conducted using monostatic systems, and therefore the modeling and analysis generally focus on echoes received in the backscattering direction. Notable exceptions are theoretical studies and experimental works on forward scattering from the swimbladders of fish (Diachok, 1999; Ding, 1997; Ye and Farmer, 1996).

To the contrary of the scenarios in laboratory experiments, acoustic scattering measurements of marine organisms in the field usually involve one or multiple uncontrolled scatterers in the sampling volume (Foote, 1991; Medwin and Clay, 1998). In this case, characteristics of the sensing system and transducer beampattern, the locations of the organisms in the beam, and the scattering properties of each individual organism jointly determine the echo signals received on the sensing system and have to be taken into account for accurate interpretation of echoes. "Echograms", or compilations of echo time-series from multiple insonifications of the same scatterer or sets of scatterers, reveal volumes with high-amplitude echo returns and are usually used to guide the focus of echo analysis. Conventional acoustic scattering researches, especially the studies of fish aggregations, rely heavily on subjective description of morphological characteristics of aggregations on the echograms, but more quantitative and objective echo analysis methods have been adopted in recent studies to make use of acoustic information embedded in the echoes (Jech and Michaels, 2006; Simmonds and MacLennan, 2006).

Sensing systems used in field experiments can also be categorized according to the directions in which the signals are transmitted and received. Downward-looking echosounders are standard systems used in fisheries applications and usually involve higher frequency signals at 10's to 100's kHz for the observation of individuals or aggregations of organisms located directly below the echosounders (Simmonds and MacLennan, 2006). Horizontally-looking systems are generally developed for research purposes and use signals at the range of 100's Hz to 10 's $\mathrm{kHz}$ for collecting synoptic data of biological aggregations across a large area (Farmer et al., 1999; Gauss et al., 
2009; Jones and Jackson, 2009; Makris et al., 2006; Revie et al., 1990; Rusby et al., 1973; Trevorrow and Pedersen, 2000). These two types of system differ primarily in the propagation paths of the transmitted and scattered signals, where direct-path propagation between the transducers and scatterers are expected for most downwardlooking echosounders, and waveguide modulation results from ocean boundaries, such as the sea surface and seafloor, is important for horizontally-looking echosounders. Stanton (2012) provides a brief discussion on the pros and cons of each of these systems.

The sections below review common techniques used for the analysis of in-situ echoes collected using downward-looking, monostatic systems that are typical for acoustic scattering studies of marine organisms.

\subsubsection{Echoes from individual marine organisms}

When the numerical density of organisms is low and the number of organisms in each sonar resolution cell is less than one, direct measurement of the backscattering from individual animals is possible by properly thresholding of the echo time-series [often referred to as "echo counting", (Medwin and Clay, 1998)]. Different from the situation in controlled laboratory experiment in which the experimental animal is located on the main axis of the transducer, the amplitudes of echoes from organisms in the field are modified depending on its location in the sonar beam. This beampattern modulation has to be removed to recover the actual echoes from the organisms. The beampattern effects can be eliminated through direct methods in which the target location in the beam is inferred by comparing the amplitudes and phases of signals received on separate sector of dual-beam or split-beam transducers, so that the scaling factor can be calculated and corrected (Ehrenberg, 1989). In cases where singlebeam transducers are used, the distribution of the actual echoes from the targets can be obtained by indirect methods that utilize deconvolution, inversion, or iterative procedures to eliminate the influence of the beam pattern (Ehrenberg, 1989; Fässler et al., 2009b; Stepnowski and Moszynski, 2000).

Analysis of echoes from individual organisms also needs to account for fluctuations 
in the echoes resulting from factors such as variations of the angle of orientation of the scatterer with respect to the incident field, behavior of the animal, movement of the transducer, etc. For echoes collected in the field, since the angles of orientation of the insonified targets is generally unknown, all data are usually pooled for a regression analysis to derive empirical models for TS prediction in conventional fisheries acoustic studies. A common form of empirical models assume a linear relationship between the size of the scatterers and the TS (e.g., Greene et al., 1991; Love, 1971, 1977; MacLennan and Menz, 1996; McClatchie et al., 1996; Wiebe et al., 1990), and a depth dependency in cases where gas-bearing organs are present in the animals body and depth-related compression is expected (e.g., Ona, 2003). For laboratory experiments where the angle of orientation of the organisms can be controlled or observed, attempts have also been made to incorporate the dependence of orientation in the regression model (e.g., Kang et al., 2005).

Different from the physics-based models introduced in Sec. 1.2.2, these regression models are derived based on empirical measurements with minimum reference to physics principles, and have been applied extensively in conventional fisheries acoustics studies owing to their relatively simple formulation (Simmonds and MacLennan, 2006). The effectiveness of these models is justified by the assumption that important acoustic scattering parameters, such as the distribution of angle of orientation and animal size, do not vary significantly in the survey area during relevant seasons, so that echo features revealed by empirical measurements are representative in the pertinent context. However, due to the same reason, the limitation of these empirical models should be recognized and cautions should be taken when model parameters are extrapolated (Fässler et al., 2008). Nevertheless, empirical measurements from these studies and the resulting prediction intervals in the regression models provide important quantitative measures for the natural variability of echoes that ought to be appreciated in the modeling and analysis of in-situ echoes from live animals (Gorska and Ona, 2003a,b; Stanton et al., 1993a) (also see Sec. 1.5). 


\subsubsection{Echoes from an aggregation of marine organisms}

For most biological aggregations encountered in the ocean, the numerical density of the organisms is usually high enough that the echoes from each animal overlap with one another. In this case, the scattering is from an ensemble of scatterers of different types, sizes and angles of orientation, insonified at different locations in the beam. Conventional analysis typically involves averaging the time-integral-pressure-squared (TIPS) of echoes from multiple insonification of the same aggregation in an gated volume, a technique coined "echo integration" in the fisheries acoustics literature (Medwin and Clay, 1998; Simmonds and MacLennan, 2006). The total scattered

pressure field resulting from $N$ scatterers in a gated volume between time $t_{1}$ and $t_{2}$ can be written as

$$
p_{\Sigma}(t)=\sum_{i=0}^{N-1} p_{i, s c a t}(t),
$$

where $p_{i, s c a t}(t)$ is the scattered pressure from the $i$ th scatterer. Therefore,

$$
\begin{aligned}
\mathrm{TIPS} & =\int_{t_{1}}^{t_{2}}\left|p_{\Sigma}(t)\right|^{2} d t \\
& =\sum_{i} \int_{t_{1}}^{t_{2}}\left|p_{i, s c a t}(t)\right|^{2} d t+\sum_{i \neq j} \sum_{j} \int_{t_{1}}^{t_{2}} p_{i, \text { scat }}(t) p_{j, \text { scat }}(t) d t
\end{aligned}
$$

Across multiple insonifications of the same set of scatterers, the cross terms fluctuate as a result of random scatterer locations in the sampling volume. Therefore, when averaged over multiple insonifications, the contribution from the first term (sum of squares) in (1.2) remain constant while the second term (sum of cross terms) vanishes with increasing number of insonifications. By relating the squares of the scattered pressure to the backscattering cross section for each scatterer, approximating the ranges from all scatterers to the transducer by their mean range, and integrating over the solid angle subtended by the transducer beampattern, the volume backscattering coefficient, $s_{\mathrm{V}}$, which represents the total scattering energy from all scatterers included in the processing gate, can be written as

$$
s_{\mathrm{V}}=n\left\langle\sigma_{b s}\right\rangle
$$


where $n$ is the number of organisms per unit volume, and $\left\langle\sigma_{b s}\right\rangle$ is the averaged backscattering cross section of a particular type of organism (Foote, 1983; Medwin and Clay, 1998), which is obtained through use of theoretical models or empirical measurements for individual scatterers as discussed in Sec. 1.2.2 and Sec. 1.3.2. When there is more than one type of scatterer in the gate, $s_{\mathrm{V}}$ is the weighted sum of the averaged backscattering cross sections of all scatterers. In practice, an equivalent logarithmic volume backscattering strength, $\mathrm{S}_{\mathrm{V}}=10 \log _{10} s_{\mathrm{V}}$, is usually used.

The requirement for (1.2) to hold is that the ping-by-ping change in the phase of each scatterer is large enough that the relative phases among the scatterers can be approximated by a uniform distribution between $[0,2 \pi]$. This change is generally induced by changes in the spatial locations or orientations of the scatterers with respect with sensing system. This assumption is generally satisfied for the frequencies commonly used in acoustic scattering studies of aggregations of marine organisms. The validity of (1.1) and (1.3) have been established in a study of the linearity and addition theorems for fisheries acoustics by Foote (1983).

The above analysis also assumes that the transmitted signals are not attenuated by the scattering and absorption of other scatterers [characterized by the "extinction cross section" (Medwin and Clay, 1998)], and that higher-order scattering among the scatterers is negligible. These assumptions may be violated in cases where the insonified aggregation is dense enough that there is excess attenuation of the transmitted signals due to extinction and that the second- or higher-order scattering becomes important due to the small spacing among scatterers. These effects can result in important consequences in the estimation of organism abundance (Foote, 1999, 1990; Foote et al., 1992; Stanton, 1983) and, in cases where coherent interference among closely- and regularly-spaced fish are important, affect the resonance characteristics of fish schools (Feuillade et al., 1996; Nero et al., 2007). 


\subsubsection{Analysis of narrowband echoes from aggregations of marine organisms}

Although the form of (1.3) is simple and can be directly applied to estimate the numerical density of monospecific aggregations, the model ensemble average of backscattering cross section, $\left\langle\sigma_{b s}\right\rangle$, depends fundamentally on the choice of key model parameters, which can significantly impact the results of echo interpretation. Examples of such parameters include the distribution of animal size and angle of orientation with respect to the incident wave. In other words, the same parametrization problem discussed in Sec. 1.2.2 persists in the analysis of echoes from aggregations of scatterers (Hazen and Horne, 2003; Jech, 2011; Lawson et al., 2006). More sophisticated application of (1.3) is also necessary for inferring the taxonomic composition of more complex aggregations.

For example, when independent echo measurements are available at multiple frequencies using narrowband signals (hereafter referred to as "multifrequency" measurements), it is possible to use linear inversion techniques to infer the distribution of the above key parameters from data. The problem can be set up by arranging multifrequency observations and model predictions in a matrix form, and solving for the distribution of unknown model parameters through linear inversion (Greenlaw, 1979; Holliday et al., 1989; Holliday, 1977; Tarantola, 2005). The accuracy of the results from such inversion methods depends highly on the spectral features available in the data and models, as well as the choice of the classes of model parameters incorporated into the formulation. When the organisms are of significantly different sizes or possess substantially different anatomical features, such as the differences between small and large zooplankton, or between fluidlike zooplankton and swimbladder-bearing fish, the strong frequency-dependent Rayleigh-to-geometric variation in the backscattering cross section contains valuable information that can be exploited by the inversion methods. However, although the inclusion of more model classes in the formulation may improve the resolution of inversion solutions, with only limited number of independent observations at different frequencies, such an inverse problem can easily 
become underdetermined without unique solutions. In practice, it is also important that model classes included in the inversion formulation are properly constrained using groundtruth information obtained through net catches or optical images (Lawson et al., 2006) and that the sensitivities of inversion results to model class choices are rigorously quantified (Jech et al., 1995).

Another class of methods seeks to partition echograms into separate areas of interest according to features in the difference between multifrequency volume backscattering strengths that are consistent with target organisms (Benoit-Bird, 2009b; De Robertis et al., 2010; Jech and Michaels, 2006). For example, the difference between the volume backscattering strengths at 38 and $120 \mathrm{kHz}$ for a zooplankton-dominated aggregation will be much larger than that for a fish-dominated aggregation. Such differences can be used as a threshold for the partition of the echogram (Higginbottom et al., 2000; Jech and Michaels, 2006; Madureira et al., 1993). Building on the results of threshold-based methods where categorical classification is made, recent studies emphasize the variation in echo measurements and uncertainty in model parameters by adopting soft classification rules, in which the conditional probability of the observations under each class is estimated before classification results are determined (Anderson et al., 2007; De Robertis et al., 2010; Woillez et al., 2012). The utilities and effectiveness of these methods varies significantly depending on the species composition, relative abundance and spatial overlap among species, as well as frequencies used in the analysis (De Robertis et al., 2010).

There remain many outstanding challenges in the interpretation of echoes collected using multifrequency narrowband signals. First of all, despite the patchy nature of marine biological aggregations (Benoit-Bird and Au, 2003; Folt and Burns, 1999), organisms across multiple trophic levels are collocated in important ecological processes such as foraging (Benoit-Bird, 2009a; De Robertis, 2002). This scenario will make multifrequency echogram partition inadequate in delivering correct information regarding the dynamics and spatial distribution of the animals. The use of probabilistic, rather than categorical, descriptions in combination with inversion methods may be more appropriate in these circumstances, but better parametrization and new 
analysis methods for echoes from mixed assemblages are clearly in demand. Furthermore, despite the success of discrimination of echoes from animals of broadly different size classes, the capability of species identification based on multifrequency measurements in the high $k a$ region is fundamentally limited by the lack of robust features in the echoes, due to the averaging, and thus smearing, of spectral features of the scattering from each individual scatterer in an aggregation (Stanton et al., 1993a). High resolution observations of the temporal evolution of the behavior and spatial distribution of organisms in the observed aggregations enabled by recent development of multibeam and broadband echosounders may provide important complementary information toward better classification results.

\subsection{Application of broadband signals in acoustic scattering studies}

The strong frequency dependency in the scattering of different types of marine organisms, such as the Rayleigh-to-geometric scattering transition, is one of the most robust features for the characterization and identification of scatterers (Medwin and Clay, 1998). Conventional acoustic scattering studies explore these spectral features in the echoes through use of multifrequency narrowband signals, but ambiguity remains in the interpretation of echo data, as have been discussed in Sec. 1.3.4.

The use of broadband signals in active acoustic sensing may reduce these ambiguities by providing continuous scattering spectra across a wide range of frequencies, and can increase the probability of capturing important spectral features that may not have been observed otherwise, such as the swimbladder resonance of fish (Stanton et al., 2010). Through pulse compression processing (Chu and Stanton, 1998; Turin, 1960), substantially-improved temporal resolution, which is approximately the inverse of the bandwidth, can be achieved. This improvement is particularly useful for resolving individual scatterers in an aggregation or identifying sources of scattering within an animal body. These advantageous properties of broadband signals have 
been used routinely in the operation and research of radar systems (Le Chevalier, 2002; Van Trees, 2001), whereas their common use in the context of acoustic scattering studies of marine organisms is relatively recent (Foote et al., 2005; Stanton, 2009), except for many early at-sea observation of fish swimbladder resonance using explosives (Chapman and Marshall, 1966; Chapman et al., 1974; Hall, 1981; Hall and Quill, 1983; Hersey et al., 1961; Holliday, 1972; Thompson and Love, 1996).

Recent progress in the use of broadband signals to study the acoustic scattering from marine organisms has largely been enabled by the advancements in the manufacturing of broadband transducers for both laboratory and field uses (Imaizumi et al., 2008; Lavery et al., 2010b; Stanton et al., 1998b, 2010). Compared to dangerous explosives, these instruments allow reproducible signals for reliable calibration and data interpretation (Stanton and Chu, 2008), as well as continuous sampling over a large region in field experiments (Lavery et al., 2012; Lawson et al., 2012; Stanton and Chu, 2010; Stanton et al., 2010). Broadband transducers have also been used in controlled laboratory experiments and have enabled the identification of dominant scattering mechanisms for a variety of marine organisms (Foote et al., 2005; Reeder et al., 2004; Stanton et al., 1998b). Insights from these experiments have formed the basis for significant developments of theoretical acoustic scattering models (discussed in Sec. 1.2.2). The application of broadband echosounders at sea also allows highresolution imaging of many biological and physical oceanographic processes, with the additional capability of systematic characterization and discrimination of the scattering sources (Lavery et al., 2010a,b; Stanton et al., 2012).

New echo features revealed through the broad spectral coverage and significantlyimproved temporal resolution offered by broadband signals has motivated the development of sophisticated data-driven and model-based echo analysis methods that were previously unachievable using narrowband signals. Several different feature extraction schemes have been applied to the broadband backscattering spectra from live zooplankton and fish measured in the laboratory and the field, and modern machine-learning algorithms have been applied to the classification of these animals and estimation of their angles of orientation with respect to the incident wave (Jaffe 
and Roberts, 2011; Martin et al., 1996; Martin-Traykovski et al., 1998a,b; Roberts

and Jaffe, 2007, 2008). The fine temporal structures from fish echoes resolved using broadband signals have also been explored for their potential of species identification (Ito et al., 2011; Matsuo et al., 2009, 2012).

On the other hand, the analysis of broadband data also poses new challenges. For example, in order to avoid problems associated with the uncertainties of the exact locations of spectral resonances in the scattering of spherical standard targets, sophisticated calibration schemes have been developed by concatenating the usable band of multiple optimized standard targets (Foote, 2006; Foote et al., 1999a), or by utilizing only the relatively stable scattering response of the front interface of the standard target [the "partial-wave" analysis, (Dragonette et al., 1981; Stanton and Chu, 2008)]. However, the potential depth dependency of a transducer's response mandates in-situ calibration at operational depth, where stable and on-axis insonification of standard targets can be difficult, especially for higher frequency transducers with narrower beamwidths. Recent availability of split-beam broadband transducers could simplify the problem, but more research is needed to establish a standardized calibration protocol. Furthermore, different from the case of narrowband echosounders, the frequency-dependent beampattern of broadband systems has to be taken into account in the calculation of volume backscattering strength (Stanton et al., 2010). The interpretation of such echoes can be challenging, especially for mixed assemblages with potentially complex spatial structures. The high ping-to-ping variability observed in broadband echoes collected in the field also calls for more quantitative description of the fluctuation of echoes.

\subsection{Statistical analysis of echo fluctuations}

While conventional studies of acoustic scattering from marine organisms focus on the analysis of mean echo energy and eliminate fluctuations through averaging, fluctuations in the echoes contain important discriminative features for the characterization of scattering sources (Olshevskii, 1967). In fact, statistical analysis of echo fluctua- 
tions has been an integral part of routine analysis procedures for many remote-sensing systems, and is an active research area for those applications. For example, the statistics of electromagnetic scattering from the sea surface is critical for maritime radar applications and rapid assessment of complicated surface wave profiles (Ward et al., 2006; Watts and Wards, 2010), and the statistics of acoustic scattering from the sea floor provide realistic representation of background for target detection purposes in reverberant underwater environments.

The analysis of echo fluctuations is generally aimed at using various statistical quantities as features for the interpretation of echo data. These include the probability density function and summary statistics of the magnitude of backscattering amplitude (or its envelope, when broadband signals are considered) or echo intensity calculated by the square of the scattering sound pressure. The relationship between echo fluctuations and the mean backscattering cross section, $\left\langle\sigma_{b s}\right\rangle$, can be understood by treating $\left|f_{b s}\right|$ as a random variable and using the relationship $\operatorname{Var}\left(\left|f_{b s}\right|\right)=\left\langle\left|f_{b s}\right|^{2}\right\rangle-\left\langle\left|f_{b s}\right|\right\rangle^{2}$ and $\left\langle\sigma_{b s}\right\rangle=\left\langle\left|f_{b s}\right|^{2}\right\rangle$ to obtain

$$
\left\langle\sigma_{b s}\right\rangle=\operatorname{Var}\left(\left|f_{b s}\right|\right)+\left\langle\left|f_{b s}\right|\right\rangle^{2}
$$

The degree to which the probability density function of $\left|f_{b s}\right|$ (hereafter referred to as "echo pdf") deviates from the Rayleigh distribution is an important feature in such analysis. Specifically, when the echoes are from a large number of random components, the echo pdf approaches the Rayleigh distribution as a result of the central limit theorem (Goodman, 1985). The presence of strong coherent component(s) in the echoes or inhomogeneities in the scatterer ensemble, on the other hand, will cause the echo pdf to possess heavy tails with strong non-Rayleigh characteristics (Jakeman and Pusey, 1976).

Motivated by the need to infer biological information through echoes, statistical analysis of echo fluctuations from marine organisms generally seeks to relate statistical properties of the echoes to the scattering properties of the animal or animal aggregations. When the scattering from individual animals is considered, echo fluc- 
tuations are associated with the scattering mechanisms of the insonified animal with respect to its angle of orientation and relative movements of different body parts. For example, at high frequencies $(k a \gg 1)$ where the scattering contribution from the facets and various parts of the fish body are important, the shape of the echo pdf measured from a single fish varies from non-Rayleigh (can be described by a Rician distribution) to Rayleigh-like as the activity level of the fish increases (Clay and Heist, 1984). The dependency of the shape of the echo pdf on the dominant scattering mechanisms for individual animals at different angles of orientation has also been observed and discussed with implications on its use for classification purposes (Stanton et al., 2004; Wiebe et al., 1990). These observations also motivated a recent theoretical investigation on the statistics of echoes from a randomly-rough, randomly-oriented prolate spheroid, whose shape is a first-order approximation of many elongated marine organisms (Bhatia et al., submitted). Although these echoes fluctuations have been recognized in TS or backscattering cross section measurements in conventional fisheries acoustic studies (see Sec 1.2.2), their influence on the empirical regression models through the evaluation of prediction intervals was generally ignored in practice until recently when probabilistic analysis of echoes became more common (Anderson et al., 2007; Brierley et al., 2004; De Robertis et al., 2010; Woillez et al., 2012).

In scenarios where echoes are measured through a directional sonar beam, the uneven weighting factor imposed by the transducer beampattern according to the locations of scatterers in the beam dramatically changes the shape of the echo pdf's and must be rigorously accounted for in the analysis (Chu and Stanton, 2010; Ehrenberg, 1972; Moszynski, 2002; Moszynski and Hedgepeth, 2000; Stepnowski and Moszynski, 2000). This random modulation, commonly referred to as the "beampattern effects", is particularly important for echoes from marine organisms measured in the field since the scatterer locations in the beam are random, instead of being controlled as in most laboratory experiments. The beampattern modulation is an important driving factor for echoes to possess strong non-Rayleigh characteristics and therefore carries significance in the characeterization of the statistics of echoes (Chu and Stanton, 2010; Ehrenberg, 1972; Moszynski, 2002; Moszynski and Hedgepeth, 2000). 
The numerical density of marine organisms, given its fundamental importance in biological oceanography, has been the most common goal of inference in statistical analysis of echoes from aggregations. Two primary approaches have been taken to takcle this problem. The first one uses summary statistics, such as the moments and ratio of moments of the echo pdf, to calculate numerical density of scatterers based on assumptions of the statistical characteristics of single scatterers and their spatial distribution (Denbigh and Smith, 1991; Wilhelmij and Denbigh, 1984). The other approach uses theoretical analysis or numerical simulation to construct parameterized echo pdf models, and estimate the numerical density of scatterers by fitting experimental or simulated data to the models (Ehrenberg, 1974; Jobst and Smits, 1974; Peterson et al., 1976; Stanton, 1985a,b). Results of these studies have shown that echo statistics can deliver information with reasonable agreement with the estimations given by echo-integration techniques, but the discriminative power of these models decreases with increasing numerical density of scatterers as a result of the echo pdf's asymptotic variation toward the Rayleigh distribution (Denbigh and Smith, 1991).

The primary advantage of echo statistics is that, by focusing on the fluctuation, rather than the mean energy in the echoes, no explicit knowledge of the backscattering cross section or TS for individual animals or calibration of system is required (Stanton, 1985a; Wilhelmij and Denbigh, 1984). The influence of the variation of various key parameters associated with the scattering from individual organisms (Sec. 1.2.2) is absorbed into model assumptions for the statistics of echoes from individual scatterers. This therefore provides an avenue for the reduction of dimensionality that can be advantageous. However, previous studies have not addressed scenarios in which more than one type of organism is present in an aggregation, a ubiquitous and important condition in biological oceanography that motivated the use of multifrequency narrowband signals and broadband signals in acoustic scattering studies (1.4 and 1.3.4). The problem of lack of discriminative power for dense aggregations is also exacerbated by the rapid increase of sampling volume and decrease of spatial resolution with increasing range away from the transducer. The fine temporal resolution achievable using the combination of broadband signals and pulse compression processing 
may mitigate this problem and improve the range of applicability for such methods. Further research is clearly needed to explore the potential advantage of broadband signals and to clarify the influence of mixed assemblages on the statistical properties of echoes.

\subsection{Thesis overview and specific topics}

Accurate interpretation of echoes relies on detailed understanding of the acoustic scattering properties of different types of marine organisms, influence of the sensors (transducers), and proper choice of signals and analysis methods capable of revealing discriminative features that vary as a function of the above and the composition of scatterers in the insonified volume (Fig. 1-1). This thesis addresses several outstanding challenges associated with each of these factors through a combination of laboratory and field experiments, theoretical modeling, and numerical simulation. A physics-based approach is taken in model development and echo interpretation, using echo features in the spectral and temporal domains as well as the statistics of echo fluctuations, with knowledge drawn from signal and probability theory and the biology of marine organisms, including anatomy and behavior. The works presented in this thesis provide new data, models, and approaches that can serve as the basis for accurate interpretation of echoes from biological scatterers in the ocean.

In Chapter 2, the orientation-dependent backscattering properties from individual live squid were studied through a combination of a controlled laboratory experiment and a series of data-model comparisons using DWBA models evaluated with different shape representations of squid. This study is motivated by the need for accurate and reliable information on the abundance, distribution, and population dynamics of squid in the ocean, which are critical for comprehensive ecological studies and fisheries management. Net-based methods that have been used conventionally to collect such information for fish species are inadequate for squid, primarily due to the effective net-avoidance capability of these rapid-moving animals (Starr et al., 1998). Active acoustic methods can provide a solution to this problem, but the applicability has been 


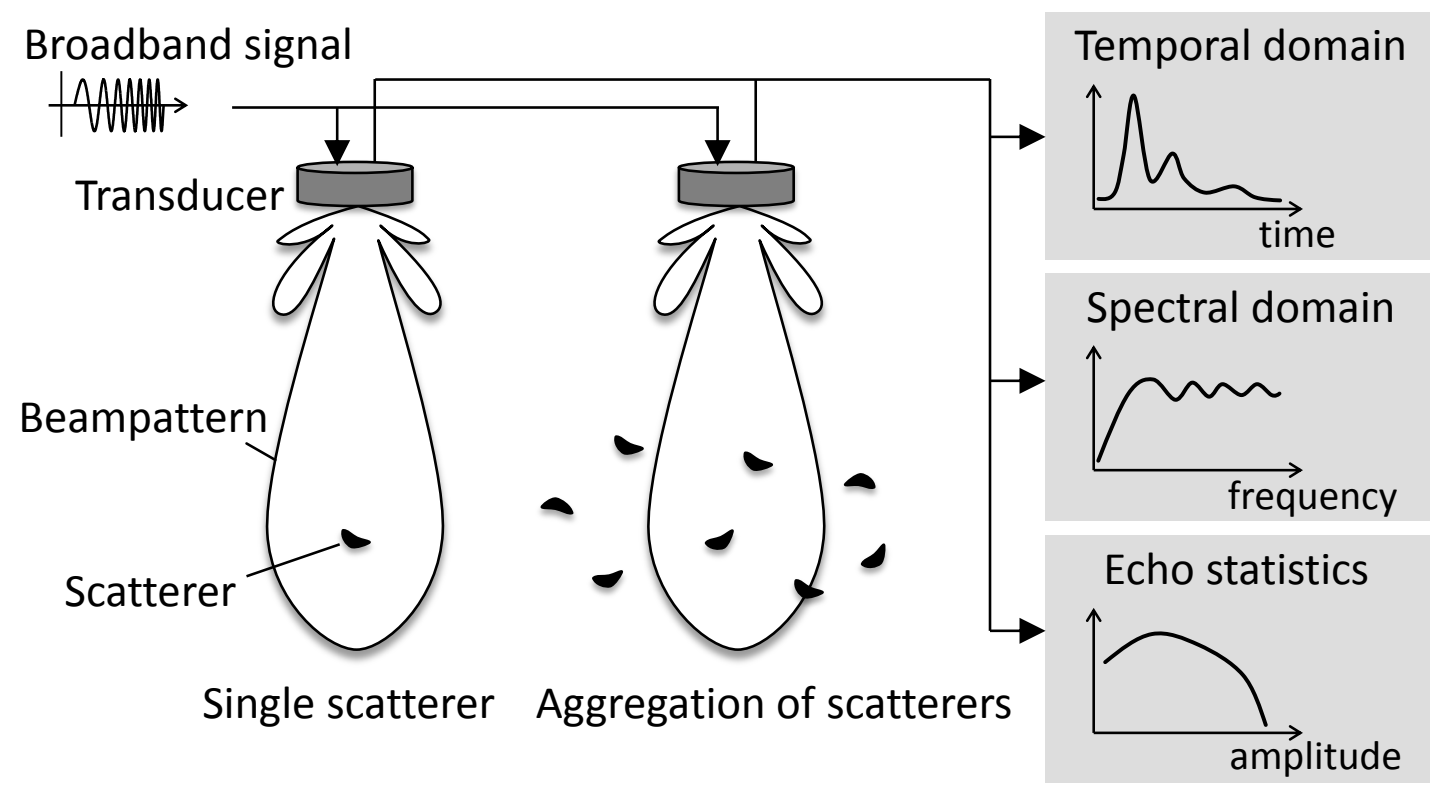

Figure 1-1: Conceptual diagram of the problems studied in this thesis and the approaches taken for the analysis of the echoes.

impaired by the long-standing issues concerning the dominant scattering mechanisms of squid. Furthermore, although several models have been proposed to predict the scattering from squid based on the hypothesized weakly-scattering material properties of squid (Arnaya and Sano, 1990; Jones et al., 2009; Kang et al., 2006; Mukai et al., 2000), a thorough verification of these models over different angles of orientation using broadband data has not been conducted.

Following similar strategies used in a series of previous studies on the backscattering of individual zooplankton, fish, and shelled animals (Reeder et al., 2004; Stanton and Chu, 2004; Stanton et al., 1998b, 2000), broadband signals and pulse compression processing were used to identify the observed dominant scattering mechanisms at all angles of orientation of squid. The sources of scattering at different angles of orientation were successfully explained using model predictions in both the temporal and spectral domains, and recommendations were given for modeling the scattering from squid in potential field conditions. Variation of the shape of the echo pdf formed by echoes collected from different ranges of angle of orientation was also examined, and 
the observations were explained based on the identified backscattering mechanisms with reference to the features of the statistics of echoes from a randomly-rough prolate spheroid (Bhatia et al., submitted). The majority of material in Chapter 2, excluding the statistical analysis of squid echoes, has been published in Lee et al. (2012), but additional figures are included to demonstrate consistent results from more than one experimental animal.

Chapter 3 and Chapter 4 focus on the development and application of narrowband and broadband echo pdf models for arbitrary aggregations of scatterers observed using a directional single-beam transducer in a direct-path geometry without interference from boundaries. This is the typical scenario encountered in field experiments where marine organisms are observed from downward-looking echosounders. The models are constructed based on physics principles and rigorously account for the influences of the system, signals, and scatterers, as well as the beampattern modulation of the receiver. These studies are motivated by the desire to establish echo statistics features, such as the degree to which the echo pdf deviates from the Rayleigh distribution and the shape of the high-amplitude tail, as new dimensions of information complementary to the spectral and temporal echo features that can be used for the interpretation of echoes. It is worth noting here that although these studies are inspired by the study of biological aggregations in the ocean, the echo statistics models developed in Chapter 3 and Chapter 4 are generally applicable to scattering signals measured from different targets in different environments using different systems, such as in the analysis of vegetation backscattering measured using radar signals.

Chapter 3 specifically addresses the use of narrowband echo pdf models in the analysis of mixed assemblages, which are defined here for cases in which more than one type of scatterer are spatially-interspersed and uniformly-distributed within the volume enclosed by the analysis window. Important examples of mixed assemblages in nature include collocated fish and zooplankton in foraging processes, forests composed of mixed vegetation, bubble plumes under sea surface, rocks overlaid on sand ripples on the seafloor, etc. (Greig-Smith, 1984; Heezen and Hollister, 1971; Onsrud et al., 2004; Woolf, 1997). The study in this chapter explores the application of discrim- 
inative echo statistics features to facilitate the interpretation of echoes from mixed assemblages that is often ambiguous using echo integration techniques (Sec. 1.3.4). An exact, physics-based narrowband model for describing the echo pdf of arbitrary assemblages was derived, implemented, and numerically validated in this chapter. This model was applied to predict echo statistics features from mixed assemblages, which were found to possess strong non-Rayleigh characteristics that can be related to the number of dominant scatterers in the aggregations. When used as an inference tool to estimated the composition of simulated mixed assemblages, this model achieved superior performance than the commonly-used mixture models (weighted sum of pdf's), sometimes by an order of magnitude. Chapter 3 repeats mostly verbatim the material in a manuscript submitted for consideration of publication in a peer-review journal (Lee and Stanton, submitted), with several additional figures included to demonstrate detailed results of model implementation.

However, the study in Chapter 3 is conducted based on the assumption of narrowband signals and is therefore incompatible to the broadband echo data collected by the recently-developed broadband echosounder systems (e.g., Lavery et al., 2010a,b; Matsuo et al., 2009; Roberts and Jaffe, 2008; Stanton et al., 2010, 2012). As the rich spectral information and substantially-improved temporal resolution provided by broadband signals have been shown to deliver invaluable information for echo analysis, the statistics of broadband echoes are likely to reveal additional useful information for the interpretation of echoes. For example, the analysis in Chapter 3 exposed the problem of degrading discriminative power of echo pdf models for dense aggregations due to the Rayleigh-like echo statistics features resulting from a large number of overlapping echoes. This problem may be mitigated by the fine temporal resolution of broadband signals achieved through pulse compression processing.

In view of the above, a numerical Monte-Carlo modeling framework was developed in Chapter 4 to predict the statistics of broadband echoes from arbitrary aggregations of scatterers. This model is capable of incorporating arbitrary frequency-dependent responses of the system, transducer beampattern, and scatterers, and thereby allows the examination of variation of echo statistics features in a variety of contexts. This 
new model was applied to analyze echoes from fish aggregations collected using a broadband echosounder in a series of acoustic backscattering experiments conducted on Georges Bank in the Gulf of Maine during 2005 - 2011 (Stanton and Chu, 2010; Stanton et al., 2010, 2012). By constraining model parameters using information derived from net catches and broadband echo spectra, the numerical density of fish estimated using the broadband echo pdf model was comparable to the density estimated using echo integration methods. This result provides a first-order evaluation of the utility and performance of echo statistics methods in analyzing in-situ echo data.

Chapter 5 provides a synoptic summary of the contributions and significance of the studies in this thesis. Recommendations of future research directions are also given, followed by a discussion on the impacts of these studies in a broader context of the application and research of active remote-sensing using human-made sonar and radar systems, and its implications in the studies of animal echolocation. 


\section{Chapter 2}

\section{Orientation dependence of}

\section{broadband acoustic backscattering from squid}

\section{$2.1 \quad$ Introduction}

Squids are ecologically and commercially important marine organisms. They support many near-shore and pelagic fisheries and transfer energy across different trophic levels through their roles as both predator and prey (Payne et al., 2006; Rodhouse, 2001; Santos et al., 2001). However, conventional net-based survey methods are inherently sparse in both space and time, and suffer from the problem of avoidance and damage to the animals. These problems are exacerbated for squid due to their highly variable abundance, rapid speed, and effective avoidance capabilities (Boyle and Rodhouse, 2005; Starr et al., 1998). Acoustic scattering techniques, on the other hand, can provide synoptic data over relevant temporal and spatial scales with high resolution (Medwin and Clay, 1998), and do not suffer from the problem of avoidance or net-induced damage to the animals, though accurate interpretation of acoustic data remains a key challenge. The combination of conventional net-tow and acoustic scattering data has the potential to provide more accurate squid stock assessment 
and distribution.

The scattering of sound from any given target is highly complex, varying strongly with the size, shape, angle of orientation relative to the incident acoustic wave, material properties of the target, and the acoustic frequency. Successful interpretation of acoustic scattering data usually requires a combination of physics-based target strength (TS) modeling, either analytical or numerical, and comprehensive laboratory measurements for verification of the model predictions (Medwin and Clay, 1998). The frequency response of a particular target provides one avenue for remote discrimination and characterization. However, many acoustic surveys continue to rely on narrowband techniques, which do not systematically provide sufficient information for accurate discrimination, classification, and characterization of the insonified scatterers. Recent advances in broadband acoustic scattering instrumentation and techniques, resulting in greater spectral coverage of the scattered frequency response, provide a new opportunity to develop more effective target discrimination and classification algorithms (Foote et al., 2005, 1999b, 2000; Lavery et al., 2010b; Stanton, 2009; Stanton et al., 2010).

Acoustic backscattering from squid has been the focus of laboratory studies for decades. Early on, it was concluded that the TS of live squid is significantly different from dead specimens, dominated by the fluid-like body, and varies strongly with the orientation of the squid relative to the incident sound wave (Arnaya and Sano, 1990; Arnaya et al., 1989; Kajiwara et al., 1990; Starr et al., 1998). Based on these findings, live squid have been used in most recent experiments, and the target strength variation with angle of orientation has been one of the primary goals of a number of studies (Benoit-Bird et al., 2008; Kang et al., 2005; Kawabata, 1999, 2001, 2005). In addition to the above narrowband measurements, a limited number of broadband measurements of scattering from squid using artificial toothed-whale echolocation signals have also been conducted on either live or dead squid (Au and Benoit-Bird, 2008; Madsen et al., 2007). However, detailed spectral analyses were not available for these studies, and data were collected only at normal and end-on incidences.

Several acoustic scattering models have been proposed for squid based on their 
fluid-like, weakly scattering properties. These models include the exact modal-series solution for a liquid prolate spheroid (Arnaya and Sano, 1990; Mukai et al., 2000), the Kirchhoff ray-mode model (Kang et al., 2006), and the distorted-wave Born approximation (DWBA) formulation applied analytically using a simple prolate spheroid shape (Mukai et al., 2000) or numerically with a realistic three-dimensional geometry (Jones et al., 2009; Lavery et al., 2002). The development and assessment of these models are particularly important for the study of squid, since direct controlled acoustic experiments are not practical for many commercially important species of squid due to the difficulties in specimen handling. Although all of the above models have been compared with narrowband experimental data, the models have not been tested over a broad range of frequencies and angles of orientation due to the lack of experimental data.

To allow the study of both the frequency and angular dependence of the acoustic scattering from squid, a controlled laboratory backscattering experiment has been conducted on live squid (Loligo pealeii) using broadband signals $(60-103 \mathrm{kHz})$ with data collected over the full $360^{\circ}$ of orientation in the lateral plane, in $<1^{\circ}$ increments. Data collected in this study have been compared to model predictions given by the analytical DWBA prolate spheroid model and the three-dimensional DWBA numerical model which takes into account inhomogeneous, fluid-like material properties, and realistic squid shape, obtained using computed tomography (CT) scans (Jones et al., 2009). The prolate spheroid model provides an assessment of the performance of this models which involves an approximation to the shape of the squid, while the flexibility of the three-dimensional model also allows digital manipulation of the squid shape to investigate the influence of the shape of different body parts, such as the arms and the fins, on the scattering.

It is generally understood that the scattering of squid is dominated by its fluid-like body construction (the muscles) with material properties very close to sea water (Iida et al., 2006; Kang et al., 2006). However, there remain questions over the scattering contribution from other body parts, including the chitinous beak and pen (gladius), eyes, internal organs such as the liver and gonads, skulls, and even the thickened 
suckers on the arms (Benoit-Bird et al., 2008; Goss et al., 2001; Madsen et al., 2007). Taking advantage of the broadband signals used in this study, pulse compression processing (Chu and Stanton, 1998) has been used to identify the dominant scattering mechanism of squid at different angles of orientation. This time-domain technique has been applied successfully in previous studies of scattering from zooplankton, fish, squid, and shells (Au and Benoit-Bird, 2008; Reeder et al., 2004; Stanton and Chu, 2004; Stanton et al., 1998b, 2000).

The above spectral, temporal, and angular characterization of squid scattering is complemented by an investigation of the statistical properties of the echoes. In contrast to the other methods that base the analysis on mean echo energy, echo statistics methods make use of the echo fluctuations as an avenue for echo interpretation (Olshevskii, 1967). Echo statistics analysis has been extensively used in a variety of aerial and underwater applications performed by radar and sonar systems (Abraham and Lyons, 2010; Ward et al., 2006; Watts and Wards, 2010). The analysis generally relies on the degree to which the distribution of echo amplitudes (echo pdf) deviates from the Rayleigh distribution as a proxy for the discrimination and classification of the sources of scattering (Sec. 1.5).

In the geometric scattering region $(k a \gg 1)$ where the backscattering from squid was measured in this study, the scattered acoustic waves can be approximated as acoustic "rays" originating from specific physical features within or at the boundaries of the organism (Stanton et al., 1998a, 2004). Since the Rayleigh distribution is the asymptotic limit of the sum of a large number of random phasors (a result of the central limit theorem, see Goodman, 1985), a small number of rays will result in a non-Rayleigh echo pdf, with the shape of the echo pdf increasingly resembling the Rayleigh distribution as the number of rays increases. Therefore, statistical features in the echoes can be used to gain insights for understanding the dominant scattering mechanisms of individual organisms (Stanton et al., 1998a, 2004).

Based on data collected in laboratory experiments, an early study showed that the features of the probability distribution of echo amplitudes (echo pdf) from individual live fish is closely related to its behavioral state, and can be described by the Rice 
distribution with the variation from Gaussian-like to Rayleigh-like for a calm fish to an active fish (Clay and Heist, 1984). Similar Gaussian-to-Rayleigh-like transition in the echo pdf was also observed for the scattering from small zooplankton to larger zooplankton observed in the field through a dual-beam echosounder, and this transition was explained as a result of the degree of variation in the phase of the echoes (Wiebe et al., 1990). A more recent study further related the observed features in the echo pdfs at different angles of orientation to the dominant backscattering mechanisms for different types of marine organisms, including both fish and zooplankton (Stanton et al., 2004). In this study, the statistics of echoes from squid have been investigated across different ranges of angles of orientation, and the results have been interpreted jointly with other temporal and spectral echo features for understanding the scattering mechanisms of squid.

This study (1) provides the first set of broadband acoustic scattering data from live squid with full coverage of angle of orientation in the lateral plane, (2) assesses the performance of two DWBA-based models, (3) identifies the observed dominant scattering mechanisms of live squid for important orientations, (4) investigates the importance of squid arm posture and fin shape in determining the scattering, and (5) reveals the strong non-Rayleigh statistical features in the echoes from randomlyoriented individual live squid. This study also gives insight into the application of the TS models under possible field conditions.

This chapter is organized as follows. In Sec. 2.2, detail of the backscattering experiment is presented. Sec. 2.3 addresses the theory and modeling of squid scattering, and results of the data-model comparison are discussed in Sec. 2.4. The statistics of squid echoes are analyzed in Sec. 2.5. A comprehensive discussion on the broadband backscattering from squid is given in Sec. 2.6, followed by a summary in Sec. 2.7. 


\begin{tabular}{cccccc}
\hline Animal \# & $\begin{array}{c}\text { Mantle } \\
\text { Length } \\
(\mathrm{cm})\end{array}$ & $\begin{array}{c}\text { Mantle } \\
\text { Width } \\
(\mathrm{cm})\end{array}$ & $\begin{array}{c}\text { Total } \\
\text { Length } \\
(\mathrm{cm})\end{array}$ & $\begin{array}{c}\text { Range of Angle of } \\
\text { Orientation Measured } \\
\left({ }^{\circ}\right)\end{array}$ & $\begin{array}{c}\text { Calculated } \\
\text { Weight } \\
(\mathrm{g})\end{array}$ \\
\hline $0807 \mathrm{a}$ & 12.2 & 3.3 & 16.2 & $803 / 742$ & 55.8 \\
$0812 \mathrm{a}$ & 7.7 & 2.1 & 12.5 & 722 & 20.7 \\
$0814 \mathrm{a}$ & 6.8 & 2.3 & 15.0 & $759.5 / 768.5$ & 15.9 \\
$0819 \mathrm{c}$ & 10.8 & 2.8 & 17.0 & 733 & 43.0 \\
$0822 \mathrm{a}$ & 11.4 & 3.0 & 18.0 & 751.5 & 48.3 \\
$0825 \mathrm{a}$ & 11.4 & 3.0 & 17.5 & 412.5 & 48.3 \\
$0826 \mathrm{~b}$ & 9.6 & 2.8 & 16.6 & 285 & 33.3 \\
\hline
\end{tabular}

Table 2.1: Dimensions and ranges of angle of orientation for the squid used in the acoustic backscattering measurements. All dimensional measurements were conducted when the animal was dead after the acoustic experiment was completed. The Total Length is the length from the tip of the mantle to the tip of the arms when the squid is placed flat on a surface. The Mantle Width is the width of the widest portion of the mantle on the dorsal side. The Mantle Length is the length between the two ends of the mantle on the dorsal side. Two numbers in the Range of Angle of Orientation Measured indicate that acoustic measurements were conducted twice on the same individual. The Calculated Weight was calculated based on the published length-weight relationship for L. pealeii (Lange and Johnson, 1981).

\subsection{Experimental methods}

\subsubsection{Squid used in the experiment}

Longfin inshore squid, Loligo pealeii, collected by the Marine Resource Center at the Marine Biological Laboratory, Woods Hole, MA were used in the experiment. This coastal epipelagic species has a long, slender body and large fins in proportion to its mantle length (Roper et al., 1984). The animals were freshly-caught ( $<1$ day) by trawl nets and kept in a tank filled with flowing chilled seawater. Healthy individuals were selected by visual inspection with the requirement that the body length has to satisfy the far-field criteria in the geometry of the experimental setup. A total of seven individuals (Table 2.1) were used and nine successful acoustic measurements were made.

Spiral computed tomography (SCT) images obtained by Jones et al. (2009) were used in this study to produce realistic digital representations of the three-dimensional shape of the squid. The SCT images were obtained for a single, live, anesthetized 
squid and a single, dead, previously frozen squid, both of the same species as those used in the experiment. These scanned images were subsequently reconstructed and interpolated onto a $0.5 \times 0.5 \times 0.5 \mathrm{~mm}$ grid for modeling operations. To investigate the scattering contributions of different body parts and construct appropriate squid shapes to facilitate data-model comparison, the shape of the fins and arms of the squid in the SCT images were further modified (Sec. 2.3.2 and Sec. 2.3.3). The volume representations used for the modeling were also scaled anisometrically and interpolated onto the same $0.5 \times 0.5 \times 0.5 \mathrm{~mm}$ grid to match the morphometric dimensions of each of the individuals measured in the experiment.

\subsubsection{Tank and instrument setup}

The acoustic backscattering experiment was conducted in an indoor flume tank at the Woods Hole Oceanographic Institution. The tank was $23 \mathrm{~m}$ long and $1.2 \mathrm{~m}$ wide on a side. The tank was filled to a depth of $1 \mathrm{~m}$ with seawater at $21^{\circ} \mathrm{C}$ [Fig. $\left.2-1(\mathrm{a})\right]$. The seawater was filtered by a 5- $\mu \mathrm{m}$ filter. The experimental setup, the tank, and the transducers, were identical to those used in Stanton and Chu (2004). Most of other instruments were the same as those used in Lavery and Ross (2007). The pulse-echo system consists of a power amplifier (custom-made at the Woods Hole Oceanographic Institution), a pair of identical transducers (Reson, Goleta, CA, TC2116), an integrated preamplifier and bandpass filter (RITEC Inc., Warwick, RI, Model BR-640A), and a National Instruments (NI, Austin, TX) data acquisition system (NI Model PXI-1000B) with an embedded computer controller (NI Model PXI-8175 running Windows 2000).

The pulse-echo system was controlled by custom-written LabVIEW software modified from the one used in Lavery and Ross (2007) to display the envelope of the compressed pulse output with background reverberation subtracted (see Sec. 2.2.5) in real-time and to control the angle of orientation of the squid through the stepper motor and the associated controller (Pontech, Rancho Cucamonga, CA, STP101). An amplitude-shaded linear chirp signal (see Sec. 2.2.4) was loaded, sent through the power amplifier, and transmitted by the transmit transducer. The raw received signal 
was pre-amplified before being recorded. The transmit signal, as measured both at the input of the power amplifier and through a -40-dB signal sampler (RITEC Inc., Model SS-40) at the output of the power amplifier, and the raw received signals (in-

cluding the echoes from the squid and reverberation from the tank) were all sampled at $2 \mathrm{MHz}$ throughout the experiment. The center-to-center separation between the transmit and receive transducers was $0.34 \mathrm{~m}$ and the target-to-transducer distance was $2.71 \mathrm{~m}$, resulting in a $7.2^{\circ}$ deviation from true backscattering. This deviation was not accounted for in the modeling.

\subsubsection{Experimental procedure}

Each squid was anesthetized by soaking the animal in a $0.1 \% \mathrm{MgCl}$ solution (Mooney et al., 2010). The time required for anesthesia and whether or not the individual would survive the following tethering procedure depended on the health condition of each squid. Approximately one in every three animals was anesthetized and tethered successfully before the acoustic backscattering experiment can be conducted. The degree to which the squid is anesthetized can be discerned by the decreasing rate of respiration and the change of the color of the animal. The animal appears pale, instead of having the usual reddish tone with flashing chromatophore, when it is anesthetized.

The anesthetized animal was pierced and suspended by a Y-shaped harness made with three 4-lb monofilament lines [Fig. 2-1(b)]. This was done by piecing the squid mantle using needles attached to hollow wires, running monofilament lines through the wires, and removing the wires so that only the monofilaments remained attached to the squid mantle. The animal was then transferred into the tank where the harness is fixed onto a tetrahedron-shaped rotation frame, consisting of three 10-lb monofilament lines, a T-shaped frame above water, and a pivot on the bottom of the tank [Fig. 2-1(a)]. This tethering system allowed free movement of the squid mantle and arms without losing control over the designated angle of orientation. All of the monofilament lines were thoroughly wetted and rubbed with soapy water before each measurement. Care was taken in the animal handling and tethering process to main- 
tain the animal underwater at all times to ensure that no air bubbles formed on the body surface or in the mantle cavity. The definition of squid orientation shown in Fig. 2-1(b) is followed throughout this paper.

The rotation frame was attached to the stepper motor controlled by the central LabVIEW program and rotated with $<1^{\circ}$ increments. The increment of rotation in each experiment varied due to expansion of the o-ring connecting the stepper motor and the tethering system. The slack of the o-ring can be partly reduced by rotating the frame for a few rounds before the acoustic measurements, but the stepping increment still varied for each experiment. The stepping increment for each experiment was obtained by dividing the total rotated angle by the total number of rotations during each set of the experiment. For each experimental animal, 15 - 17 acoustic pings were collected at each angle of orientation through two full rotations $\left(720^{\circ}\right)$. It is observed that the rotation frame and the harness required approximately two seconds to settle into the new position. Therefore, in all subsequent analysis the echoes resulted from the first two pings at each angle of orientation are discarded. The experimental animals were out of anesthesia and alive during the acoustic measurements. In some cases the animal died before a full rotation was completed (Table 2.1) and the experiment was aborted.

\subsubsection{Acoustic signal analysis and calibration}

A chirp signal with a frequency span of $45-105 \mathrm{kHz}$ was used as the transmit signal. The amplitude of the transmit signal was shaded so that the overall system response was more uniform in the spectral domain (Fig. 2-2). The amplitude shading was created by modifying the inverse of the envelope of received signals when an unshaded linear chirp signal was transmitted between the transducers when they were set up in the calibration mode (see below). The best amplitude shading was selected by trial and error with the following criteria: 1) a flatter spectrum is achieved in the received calibration signal, and 2) the autocorrelation function of the received calibration signal has moderate mainlobe width and low sidelobe levels. The first criterion was chosen in order to minimize the occurrence of frequency regions where 


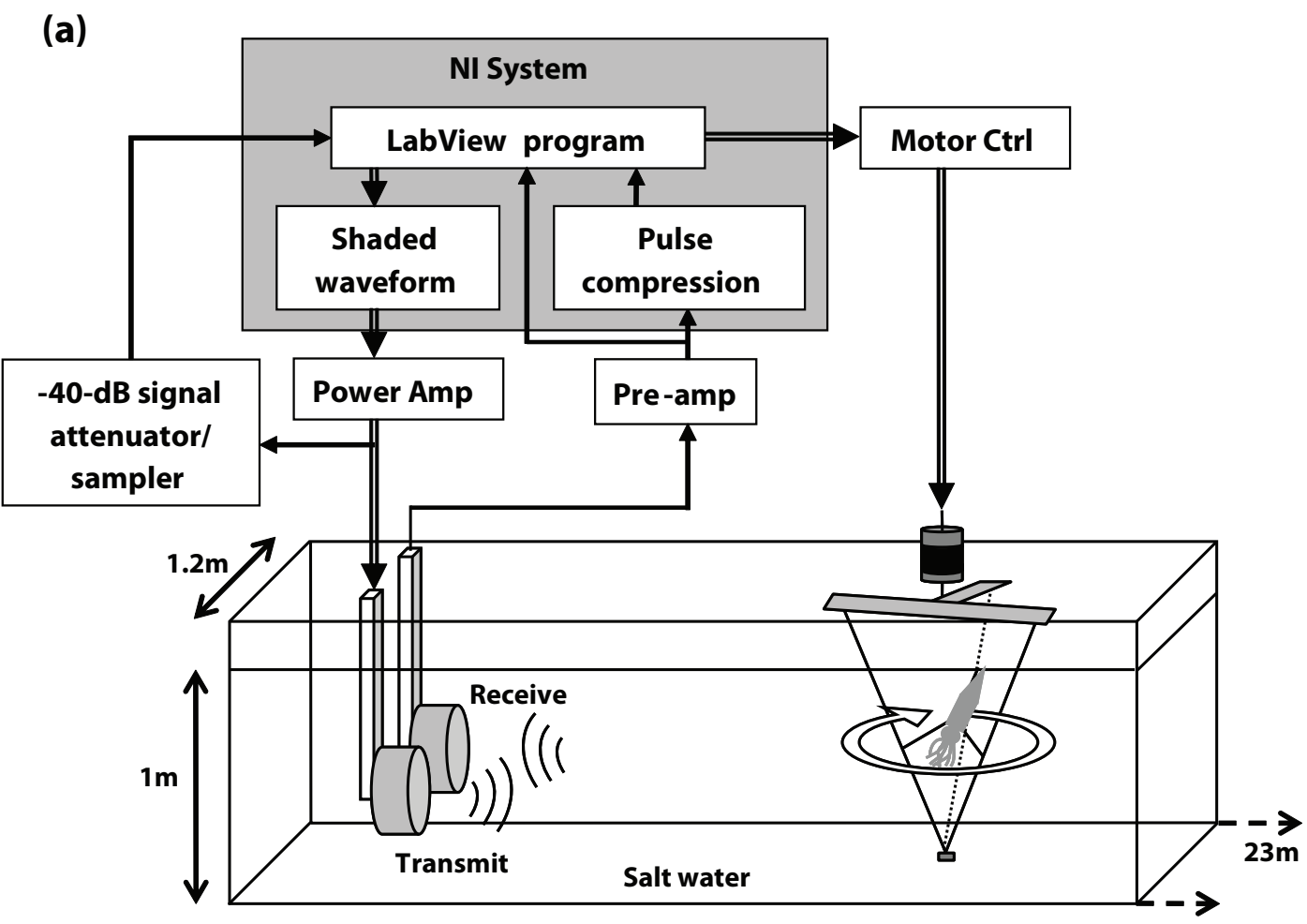

(b)

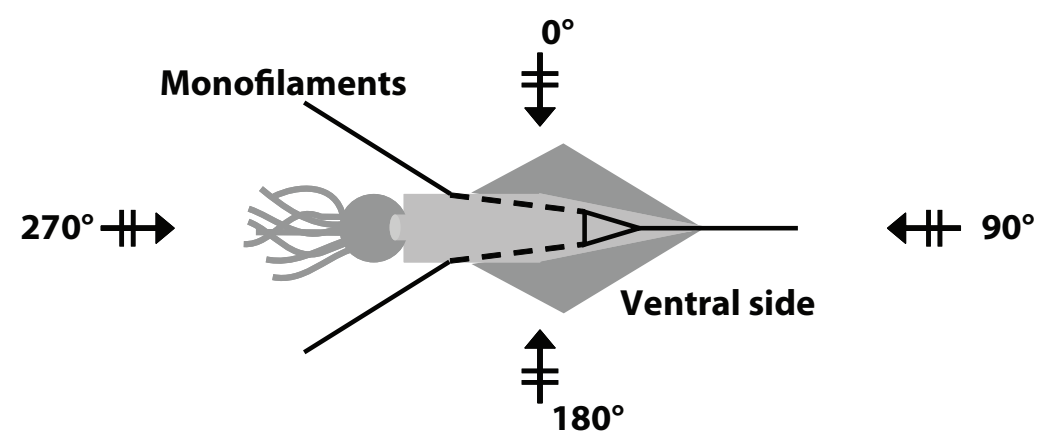

Figure 2-1: (a) The pulse-echo system and experimental setup for the laboratory measurements of scattering from squid as a function of angle of orientation. The shaded box represents the NI (National Instruments, Inc.) system containing the central LabVIEW control program. (b) Tethering system used in the experiment and the definition of angle of orientation relative to incident acoustic signal. Solid lines represent monofilament lines outside of the squid body. Dashed lines represent monofilament lines running through the mantle cavity. 
the frequency response changes rapidly, which tend to cause errors in the calibration. The second criterion was chosen to facilitate the interpretation of echo features in the time domain (see Sec. 2.6.4). Combined with the frequency-dependent noise levels, the selected transmit signal resulted in a usable band of $60-103 \mathrm{kHz}$.

The system was calibrated using the procedure described in Stanton et al. (1998b). This method involves separating the transducers, aiming them toward each other and measuring the signals as a result of the acoustic pulse traveling along the direct path between the two. Note that the amplitude of the transmit signal was significantly reduced during calibration to avoid saturation of the electronics. The optimal level of the transmit signal during calibration was also selected by trial and error, since distortion of the transmit signal can be significant when the amplitude was too low. The calibration was performed both before and after the squid measurements.

Taking advantage of the broad signal bandwidth, pulse compression techniques were used to identify the dominant scattering mechanisms of live squid (Chu and Stanton, 1998). The deviation in the compressed pulse output (CPO) envelope from the idealized matched-filter output contains information regarding the scattering properties of the target. Pulse compression processing of broadband signals has the advantage of increasing the time-domain resolution and signal-to-noise ratio (SNR). This is of particular importance for identifying the dominant scattering mechanisms for weak scatterers, such as squid.

In this experiment, the received echo signal was compressed in time by crosscorrelating the echo with the received calibration signal. The mainlobe width of the envelope of the autocorrelation function [Fig. 2-2(d)] limits the finest spatial resolution achievable by the system. The normalized height of the first sidelobe is 0.321. Sidelobes can introduce spurious artificial echoes in the analysis and must be considered carefully when interpreting scattering features. 
(a)

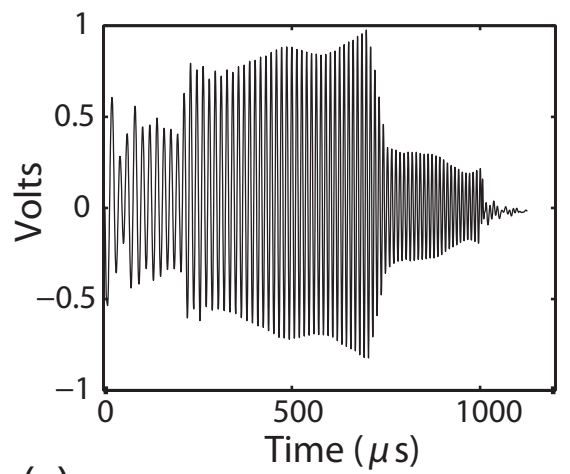

(c)

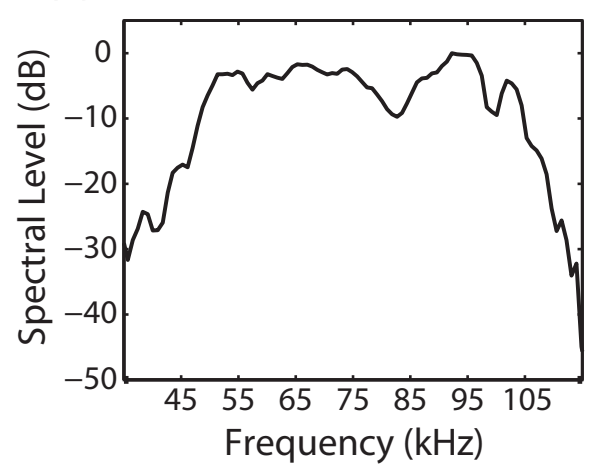

(b)
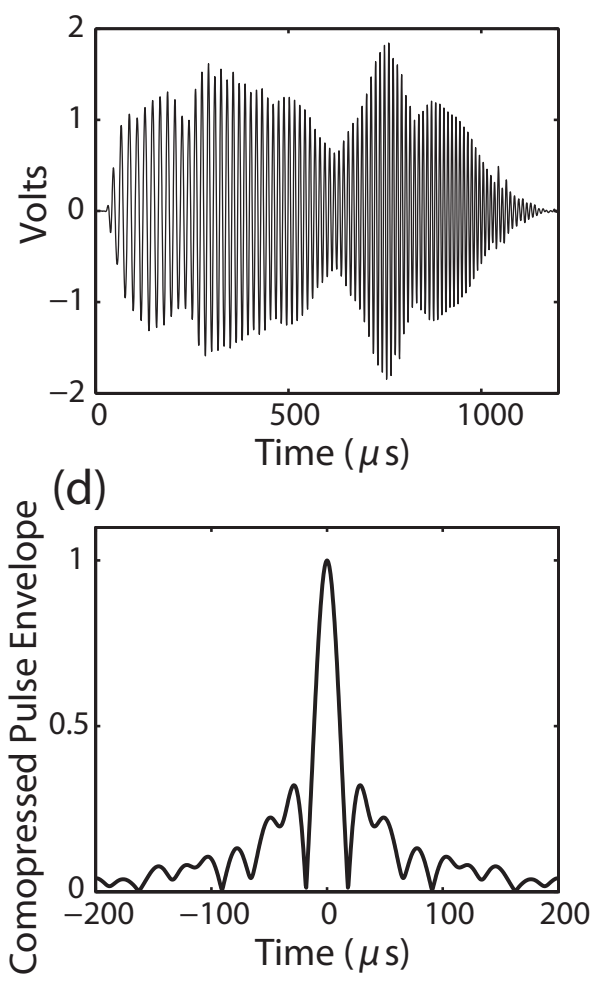

Figure 2-2: (a) Transmit signal measured at the output of the power amplifier. (b) Received calibration signal. (c) Spectrum of the received calibration signal. (d) Envelope of the autocorrelation function of the received calibration signal, normalized to the peak maximum at $0 \mu \mathrm{s}$. 


\subsubsection{Subtraction of background reverberation and control of data quality}

Because the experiment was conducted in a long, narrow tank, background reverberation had to be subtracted off to identify and isolate the echoes from the squid. Two sets of 200 pings of background reverberation (with no squid in the tethering system) were collected immediately prior to the acoustic measurements. Another 200 pings of

background reverberation were collected immediately after the acoustic measurement, when the situation permitted.

One set of the pre-experiment background reverberation signals were coherently averaged and stored as the background reverberation reference. During the backscattering experiment, unmodified raw signals consisting of both the squid echoes and background reverberation were collected. The echoes from the squid were isolated by subtracting the background reverberation reference from the raw receiving signals. It was also observed that the background noise reverberation drifted with time during each measurement, likely due to the leaking of the flume tank that caused small variation in the water depth, which could result in small deviation in the reverberation in the tank.

Reverberation signals other than those used to form the background reverberation reference were used to establish a background noise threshold for controlling the data quality of acoustic measurements. This frequency-dependent background noise threshold was obtained by taking the median value of the 200 pings of background reverberation on a frequency-by-frequency basis, after subtracting out the background reverberation reference. A signal-to-noise ratio of $6 \mathrm{~dB}$ was imposed to control the data quality: all TS measurements smaller than $6 \mathrm{~dB}$ above the threshold was considered unacceptably noisy and discarded. TS measurements within the frequency range of $71.5-82 \mathrm{kHz}$ were also discarded, because this frequency band was observed to be constantly noisy throughout the experiments. The SNR in this band was especially low at off-normal angles of orientation. 


\subsection{Acoustic backscattering theory and modeling}

\subsubsection{Basic definitions of acoustic backscattering quantities}

Acoustic scattering from an object in the far-field can be expressed as

$$
P_{\text {scat }}=P_{0} \frac{e^{i k r}}{r} f
$$

where $P_{0}$ is the pressure amplitude of the incident wave, $r$ is the distance from the object to the receiver, and $f$ is the scattering amplitude. The scattering amplitude fully describes the acoustic scattering characteristics of a target and is a measure of the efficiency with which a target scatters sound. It is a function of the acoustic wavenumber $k(=2 \pi / \lambda$, where $\lambda$ is the acoustic wavelength $)$, and the target's shape, size, angle of orientation, and material properties, such as the mass density $\rho$, and sound speed $c$.

As the dynamic range of the scattered signals is typically very large, a logarithmic measure of the backscattering amplitude is used, defined as target strength (TS), expressed in units of decibels $(\mathrm{dB})$ relative to $1 \mathrm{~m}^{2}$, and given by TS $=10 \log _{10}\left|f_{b s}\right|^{2}=$ $10 \log _{10} \sigma_{b s}$, where $\sigma_{b s} \equiv\left|f_{b s}\right|^{2}$ is the differential backscattering cross-section, and $f_{b s}$, or backscattering amplitude, is the scattering amplitude evaluated in the backscattering direction.

\subsubsection{Distorted-wave Born approximation for acoustic backscat- tering: Application to squid}

The backscattering amplitude for any weakly scattering object can be modeled in the far-field using the distorted-wave Born approximation (DWBA) in which the total pressure field within the scatterer is approximated by the unperturbed incident wave field, with the wavenumber replaced by the wavenumber inside the scatterer (Chu et al., 1993; Morse and Ingard, 1987; Stanton et al., 1993a). With this approximation 
the backscattering amplitude can be written as

$$
f_{b s}=\frac{k_{1}^{2}}{4 \pi} \int\left(\gamma_{\kappa}-\gamma_{\rho}\right) e^{2 i \mathbf{k}_{\mathbf{v}} \cdot \mathbf{r}_{\mathbf{v}}} d \mathrm{v}
$$

In the above formulation, the subscript "1" indicates parameters of the surrounding medium, while the subscript "v" indicates parameters of the scattering body. The term $\mathbf{k}_{\mathbf{v}}$ is the wavenumber vector within the scattering volume, and $\mathbf{r}_{\mathbf{v}}$ is the position vector of any volume element. The terms $\gamma_{\kappa}$ and $\gamma_{\rho}$ are defined in terms of the compressibility $\kappa$ and density $\rho$, and can be written in terms of the density contrast $g_{\mathrm{v}}\left(=\rho_{\mathrm{v}} / \rho_{1}\right)$ and sound speed contrast $h_{\mathrm{v}}\left(=c_{\mathrm{v}} / c_{1}\right)$ between the medium and the scattering object, i.e.,

$$
\gamma_{\kappa} \equiv \frac{\kappa_{\mathrm{v}}-\kappa_{1}}{\kappa_{1}}=\frac{1-g_{\mathrm{v}} h_{\mathrm{v}}^{2}}{g_{\mathrm{v}} h_{\mathrm{v}}^{2}}
$$

and

$$
\gamma_{\rho} \equiv \frac{\rho_{\mathrm{v}}-\rho_{1}}{\rho_{\mathrm{v}}}=\frac{g_{\mathrm{v}}-1}{g_{\mathrm{v}}}
$$

The integral in (2.2) can be solved analytically for simple objects, such as spheres and cylinders (Stanton et al., 1998a), and is particularly useful for numerically modeling the scattering from bodies with arbitrary shapes (Lavery et al., 2002) and material properties (Jones et al., 2009). In this study, two DWBA-based models are compared: (1) an analytical model with a simple geometry (smooth prolate spheroid) and homogeneous material properties, and (2) a numerical model which involves three-dimensional digitization of the squid and inhomogeneous material properties.

\section{Analytical DWBA prolate spheroid model}

The prolate spheroid geometry is a first-order approximation to the elongated shape of the squid (Arnaya and Sano, 1990; Mukai et al., 2000). The analytical DWBA solution for a prolate spheroid geometry [derived by D. Chu and given in Johnson 
(1993)] is reproduced here for reference:

$$
f_{b s}=\frac{k_{1}^{2} a_{p s}^{2} L_{p s}\left(\gamma_{\kappa}-\gamma_{\rho}\right)}{2} \frac{j_{1}\left(k_{\mathrm{v}} \sqrt{4 a_{p s}^{2} \sin ^{2} \theta+L_{p s}^{2} \cos ^{2} \theta}\right)}{k_{\mathrm{v}} \sqrt{4 a_{p s}^{2} \sin ^{2} \theta+L_{p s}^{2} \cos ^{2} \theta}}
$$

where $a_{p s}$ is the semi-minor axis (equatorial radius), $L_{p s}$ is the major axis (twice the polar radius), $\theta$ is the polar angle from the major axis, and $j_{1}$ is a spherical Bessel function of the first kind of order one.

In this study, the width $\left(2 a_{p s}\right)$ of the prolate spheroid was set to match the measured maximum width of each squid used in the experiment. The length of the prolate spheroid, $L_{p s}$, was determined by matching the total volume of the homogeneous (no sea-water-filled cavities in the mantle cavity) squid digital representation to the volume of the prolate spheroid. Detail of the inhomogeneities in the squid body and the scaling issues can be found in Jones et al. (2009). Note that to allow direct comparison of results to Jones et al. (2009), the digital volume representation of squid used for equivalent volume calculation was the arms-folded hybrid squid shape without modifications to the fins (see below).

\section{Three-dimensional DWBA numerical model}

The three-dimensional DWBA numerical model calculates the scattering response of an arbitrarily-shaped inhomogeneous object by numerically integrating the phase change contributed by local material property variation over a digital volume representation (Jones et al., 2009; Lavery et al., 2002). This is of particular interest in modeling the acoustic scattering from squid, since the outer shape is complex, particularly in the vicinity of the arms, and the body contains sea-water-filled cavities.

The SCT images obtained by Jones et al. (2009) were used as the baseline digital representations of squid in this study. The arms-splayed squid shape was taken directly from the actual scan of the anesthetized specimen [Fig. 2-3(a)], while the arms-folded squid shape was constructed by hybridizing the arms of the dead specimen with the mantle of the anesthetized specimen [Fig. 2-3(b)]. The orientation 
(a)

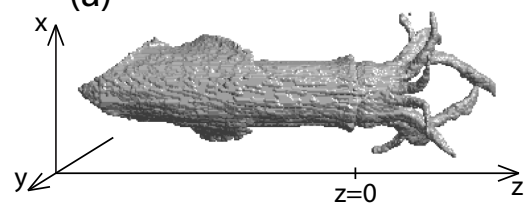

(c)

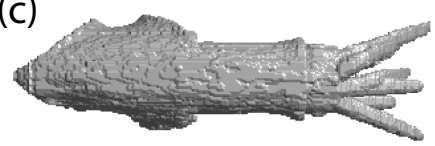

Original asymmetrical fins (d)

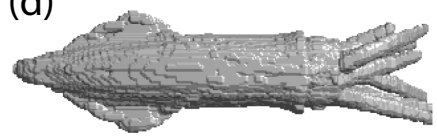

Symmetrical fins (b)

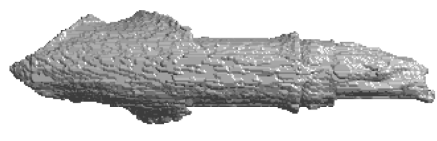

(e)

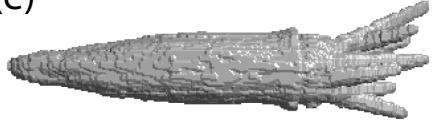

No fins

Figure 2-3: Various squid shapes used in this study: (a) the arms-splayed and (b) arms-folded squid shape without modification of the fins or randomization of the arms. (c-e) examples of hybrid randomized squid shapes with three different shapes of the fins (see Sec. 2.3.3 for detail of the fins).

of the squid in the digital representation was such that the center line of the mantle was parallel to the $z$-axis of the digital volume, with the $x$-y plane representing cross-sectional slices along the squid's longitudinal axis.

In addition to the above manipulations, the shape of the arms and the fins were further modified for modeling purposes in this study. Unless otherwise specified, the mantle volume used in the following three-dimensional DWBA numerical modeling was the mantle of the anesthetized specimen with the fins digitally removed (see Sec. 2.3.3), beause the modeling results using this mantle shape gave the best agreement with the experimental data (see Sec. 2.4.2). To facilitate data-model comparison, the mantle width and total length of the digital squid volume were scaled to match the maximum mantle width and total length, respectively, of the experimental animal.

\section{Modeling parameters}

In modeling the scattering of weak scatterers such as squid, it is known that small variations of $g$ (density contrast) and $h$ (sound speed contrast) can give rise to TS variation as large as $20 \mathrm{~dB}$ (Chu et al., 2000). However, there are no measurements available for the material properties of $L$. pealeii. Therefore, the tissue material properties of the Japanese flying squid (Todarodes pacificus) $(g=1.043, h=1.053$, 
Iida et al., 2006; Jones et al., 2009) were used instead for both the analytical DWBA prolate spheroid model and the three-dimensional DWBA numerical model in this study.

Another important modeling consideration is the digitization resolution of the modeled scatterer volume (characterized by the maximum dimension of the digitization voxel, $l_{\mathrm{V}}$ ) compared to the acoustic wavelength. A value of 20 for the ratio $\lambda / l_{\mathrm{V}}$ is generally required for properly estimating the acoustic scattering of fluid-like elongated zooplankton (Stanton and $\mathrm{Chu}, 2000$ ). Based on this principle, the numerical model output was constrained below $150 \mathrm{kHz}$, corresponding to a $\lambda / l_{\mathrm{V}}$ ratio of 20 with the digital volume resolution of $l_{\mathrm{V}}=0.5 \mathrm{~mm}$. The highest frequency of the chirp signal used in the experiment was $105 \mathrm{kHz}$, which resulted in $\lambda / l_{\mathrm{V}}=28.57$.

\subsubsection{Model predictions}

The comparison of the model predictions given by the analytical DWBA prolate spheroid model and the three-dimensional DWBA numerical model using realistic squid shapes illustrates the baseline difference between models with simple versus complicated geometries. In addition, the three-dimensional DWBA numerical model has made it possible to investigate the contribution from individual body parts by digitally modifying the shape of the squid volume. Of particular importance are the shapes of the fins and the arms, which were modified or randomized to obtain the best agreement with the data (Figs. 2-4 to 2-11).

\section{Comparison of model predictions for the analytical DWBA prolate spheroid model and the three-dimensional DWBA numerical model}

The TS predictions given by the analytical DWBA prolate spheroid model and the three-dimensional DWBA numerical model are compared for both the angular dependence (Fig. 2-4) and the frequency response (Fig. 2-5). At normal incidence, the TS predictions for both models reach the maximum with comparable values. When the incident angle deviates from normal incidence, the analytical DWBA prolate spheroid 


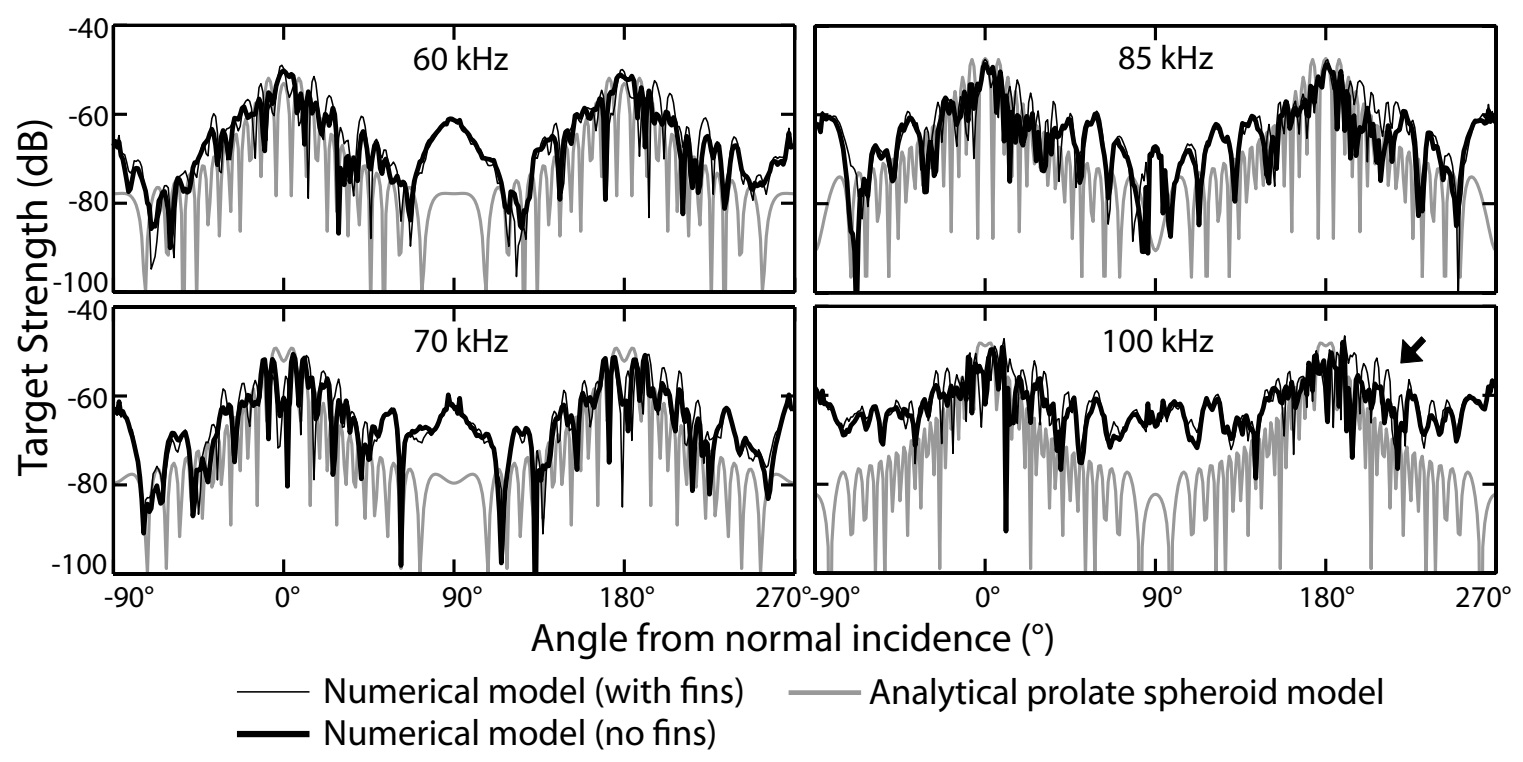

Figure 2-4: TS prediction versus angle of orientation at four frequencies $(60,70,85$, $100 \mathrm{kHz}$ ) for the three-dimensional DWBA numerical model using arms-folded squid shapes with and without the fins, and the analytical DWBA prolate spheroid model. The arrow indicates the scattering contribution from the fins.

model predictions drop much more rapidly than the three-dimensional DWBA numerical model predictions.

The analytical DWBA prolate spheroid model predictions also contain structured nulls in both the TS versus frequency and TS versus angle responses (Figs. 2-4 and Fig. 2-5), produced by constructive and destructive interference accentuated by the smoothness and symmetrical shape of the prolate spheroid. In addition, the analytical DWBA prolate spheroid TS predictions are slightly higher than those of the three-dimensional DWBA numerical model in the Rayleigh scattering region at all angles of orientation (Fig. 2-5). This is due to the fact that the prolate spheroid was scaled by matching the spheroid volume to the volume of homogeneous digital squid representation with the fins. This volume is larger than the volume of the inhomogeneous, no-fin squid shape used in the three-dimensional DWBA numerical model. 


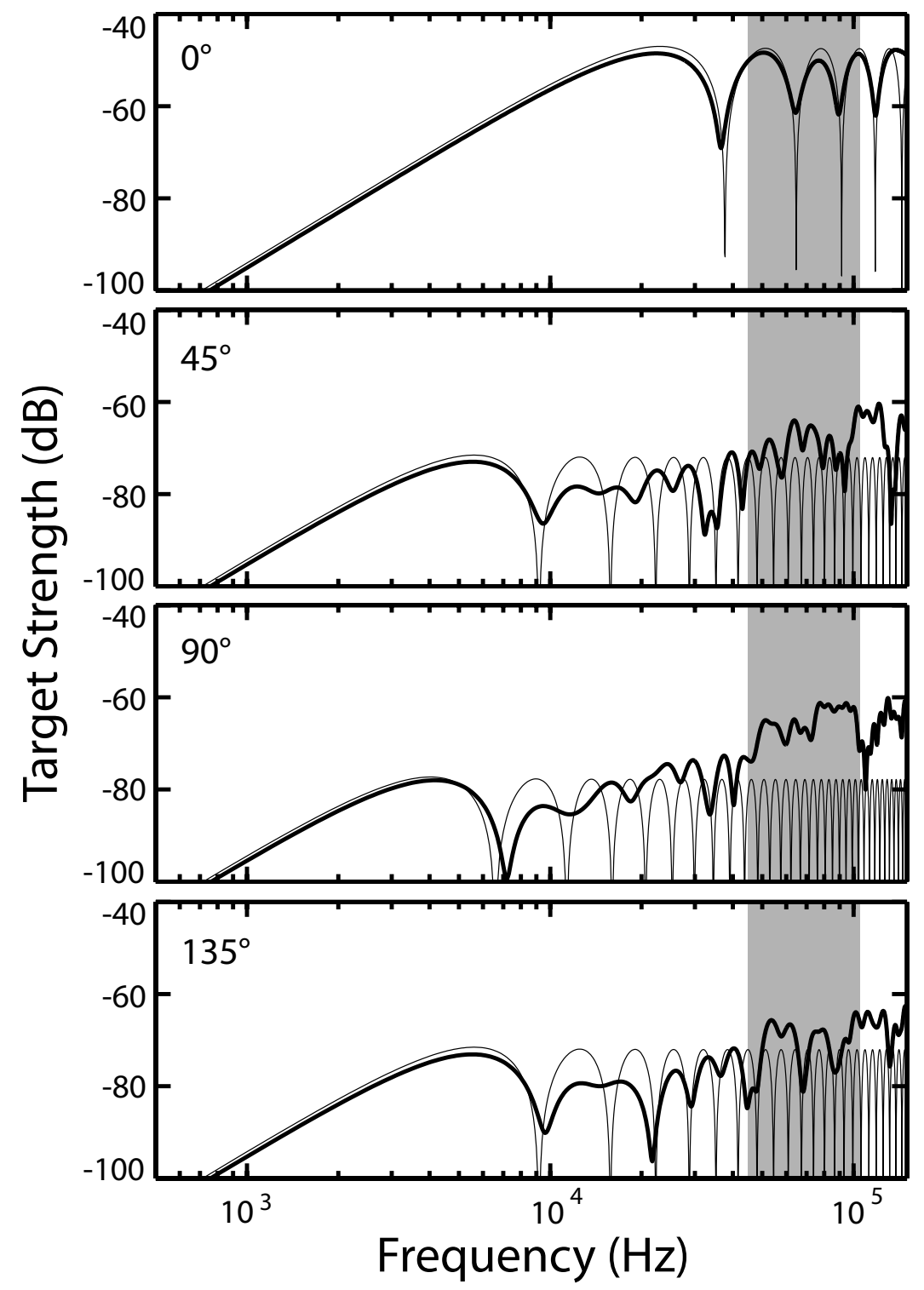

— Arms-folded numerical model (no fins)

\section{Analytical prolate spheroid model}

Usable band in the experiment

Figure 2-5: TS predictions versus frequency for the three-dimensional DWBA numerical model using arms-folded squid shape and the analytical DWBA prolate spheroid model at four angles of orientation $\left(0^{\circ}, 45^{\circ}, 90^{\circ}, 135^{\circ}\right.$ from normal incidence). The usable band (gray area) in the experiment lies entirely in the geometric scattering region. 


\section{Contribution of individual body parts}

The flexibility of the three-dimensional DWBA numerical model in incorporating complicated geometries facilitates the investigation of the scattering contributions from individual squid body parts, such as the fins and the arms. To understand the impact of the fins on the backscattering, numerical model predictions were made using the same folded arms but with the original asymmetric fins and with the fins digitally removed (Fig. 2-4). The scattering contribution of the fins is most prominent at the "shoulders" of the curve around $20^{\circ}-40^{\circ}$ from normal incidence for higher frequencies (indicated by the arrow in Fig. 2-4), and is less important at angles far from normal incidence. The asymmetric scattering pattern on either side of normal incidence is the result of the asymmetric shape of the original fins on either side of the squid.

The posture of the arms also has a significant effect on the scattering prediction across all angles of orientation (Fig. 2-6). In this figure, the model compressed pulse output (CPO) envelope was produced by cross-correlating the model impulse response with the autocorrelation function of the transmit signal (Chu and Stanton, 1998). The model impulse response was obtained by applying an inverse Fourier transform on the model spectra from $100 \mathrm{~Hz}$ to $150 \mathrm{kHz}$, in $100 \mathrm{~Hz}$ increments. In the case of the analytical DWBA prolate spheroid model, the backscattering contributions from the front and back interfaces of the prolate spheroid with respect to the incidence field are clearly shown [Fig. 2-6(a)]. For the three-dimensional DWBA numerical model, a strong sinusoidal pattern corresponding to the squid arms is observed in the modeled CPO envelopes in both plots. The scattering from the arms is stronger in the armssplayed case [Fig. 2-6(b-c)]. Since the two shapes only differ in the arm postures and have the same mantle shape, this result shows the importance of the arm posture on the acoustic scattering. A very faint secondary sinusoidal pattern was also observed resulting from scattering originating at the tail region of the squid, which is analogous to the scattering contribution from the back interface of the prolate spheroid. 
(a)

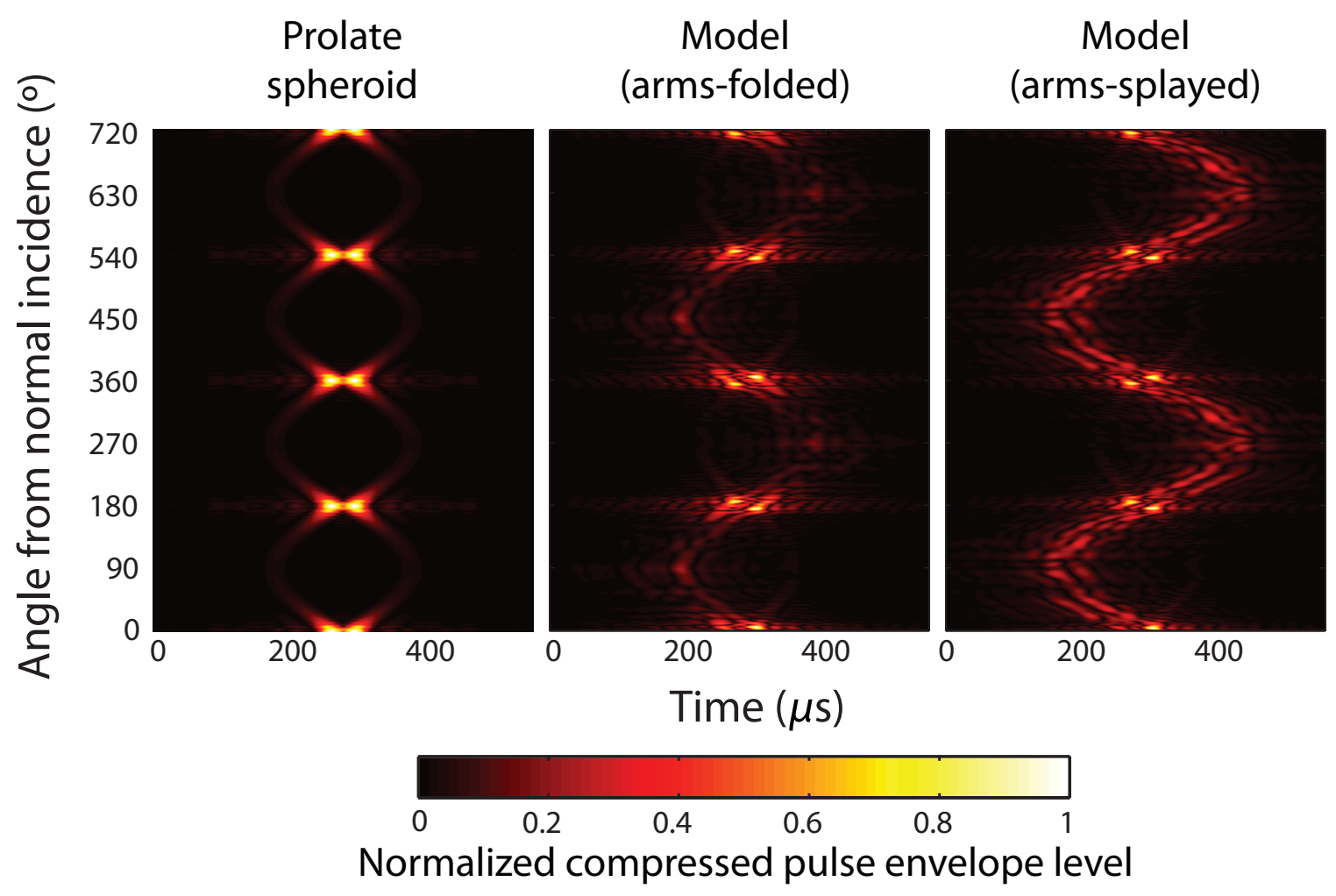

(c)
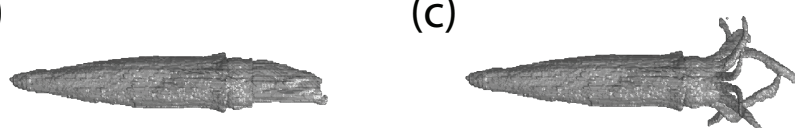

Model (arms-splayed) Normalized compressed pulse envelope level

Figure 2-6: Compressed pulse output envelope of (a) the analytical DWBA prolate spheroid model, and the three-dimensional DWBA numerical model using two fixed squid shapes through two full rotations $\left(720^{\circ}\right)$ : (b) the arms-folded configuration, and (c) the arms-splayed configuration. The compressed pulse output envelopes are normalized to the maximum envelope value in each of the plots. The symmetric sinusoidal pattern in (a) corresponds to the front and back interface of the prolate spheroid with respect to the incidence field, and the strong sinusoidal pattern in (b) and (c) corresponds to the location of the squid arms during the rotation. 


\section{Randomized squid shape}

Based on the above observation that the numerical model predictions are highly sensitive to the exact shape of the arms and the fins, these were digitally modified to produce a set of squid shape representations which are reasonably close to the squid shape during the experiment. Fifteen realizations of arms were generated to resemble the "loosely-folded" arm posture of the squid resting in the harness [Fig. 23(b)]. Three fin shapes were used in combination with these randomly generated arms to produce a set of hybrid randomized squid shapes to facilitate the data-model comparison.

The shape of each randomized arm was determined by three points: the initial, middle, and end points in the three-dimensional space. The space was defined such that the $z$-axis is parallel to the longitudinal axis of the squid body, with $z=0$ being the surface joining the arms and the mantle [Fig. 2-3(c-e)]. The $(x, y)$ positions were used to describe the transverse position on a given height of $z$. Eight initial $(x, y)$ arm positions were manually chosen on the $z=0$ plane to keep the initial arm positions biologically realistic. The positions of the middle and end points were randomly generated within a pre-defined area on the $x-y$ plane. These areas were defined according to the initial position of each arm to keep the arm shape natural. A spline function was then fitted for the three points of each arm. The arm length was also generated randomly within a biologically reasonable range. Consecutive $(x, y, z)$ points on each arm were then generated according to the spline along the height of the arm. These arm curves were then filled by individual "discs" with decreasing radius toward the tips of the arms. The rim of the discs was randomly roughened to create roughness on the arm surface. The total volume of the arms generated by this procedure was approximately $11-14 \%$ of the total squid volume, which is comparable to the arm/body volume ratio for the two original sets of SCT squid images described in Sec. 2.2.1.

Three fin shapes were used: (I) the original asymmetric fins, (II) artificially generated symmetric fins, and (III) no fins [Fig. 2-3(b)]. Fin volume (I) was directly 
obtained from the mantle portion of the SCT images of the anesthetized squid. Fin volume (III) was obtained by digitally removing the fins from volume (I). Fin volume (II) was a hybrid volume consisting of a pair of artificially edited symmetric fins and volume (III). The symmetric fins were created by producing a pair of mirror images of a scaled version of a fin retrieved from the SCT images of the dead, frozen squid. These three shapes will be referred to as "original-fins", "symmetric-fins", and "no-fins" mantles throughout the remainder of this paper.

\section{Addition of noise to model predictions}

In addition to the variations of the arms and the fins, to make valid comparisons with the experimental data, frequency-dependent noise was added to the scattering amplitude predictions for all of the randomized models to achieve valid data-model comparison (Fig. 2-7). The amount of noise added for a given frequency was calculated based on the background noise level measured during the acoustic measurement (Sec. 2.2.5). The mean and standard deviation of the the real and imaginary part of the scattering amplitude for the background reverberations were calculated. Normally-distributed random numbers using these mean and standard deviation values were then generated independently to construct the real and imaginary parts of the noise. The final noise-added model predictions were produced by coherently adding the noise-free model predictions and the complex noise:

$$
f_{b s, m n}=f_{b s, m}+f_{b s, n}
$$

where $f_{b s, m n}$ and $f_{b s, m}$ are the model backscattering amplitude with and without

noise added, respectively, and $f_{b s, n}$ is the random complex noise. The effect of noise addition is more prominent at angles away from normal incidence with lower predicted TS [indicated by the brackets in Fig. 2-7(b)]. 


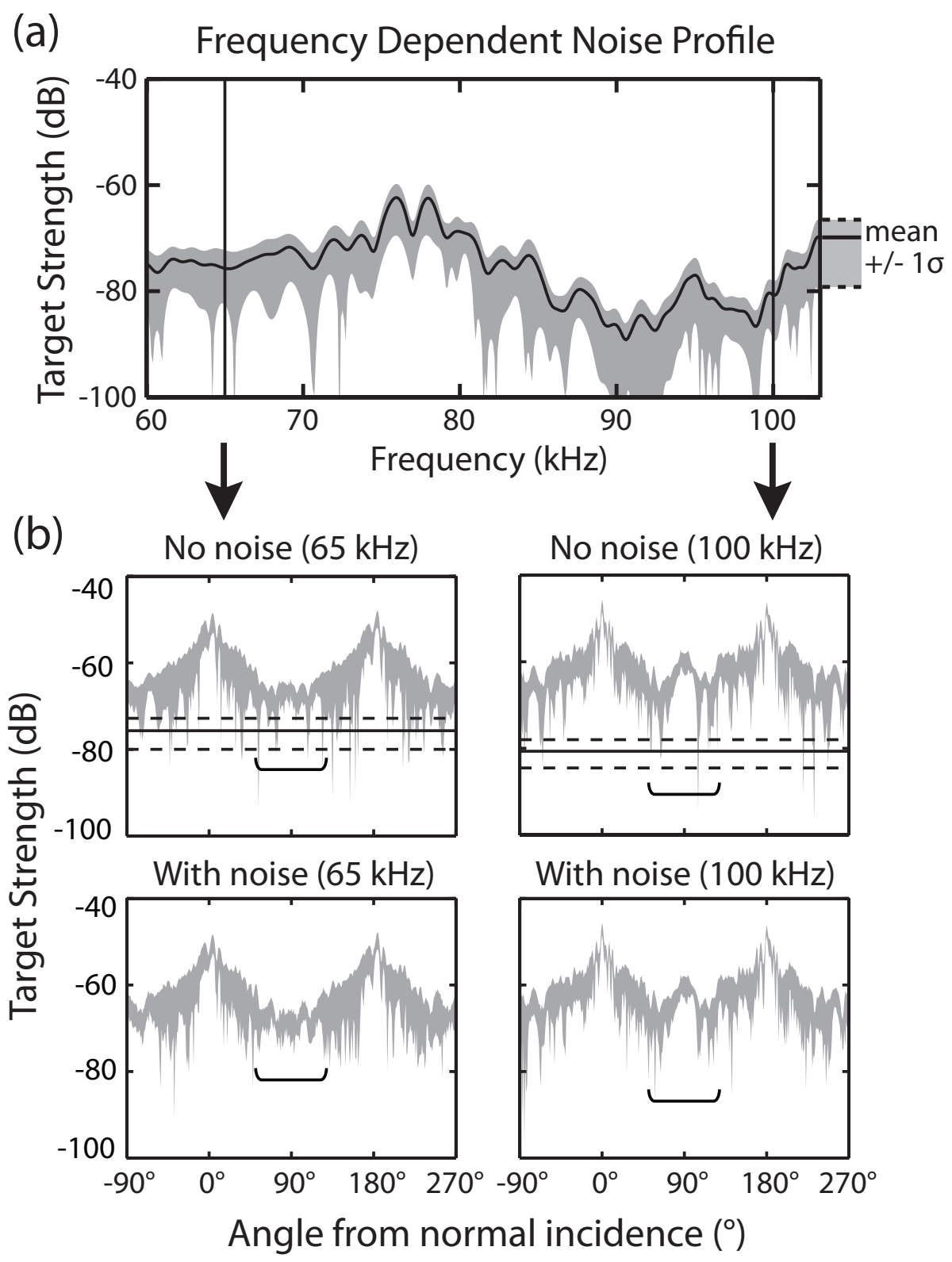

Figure 2-7: Noise addition procedure for model predictions. (a) The frequency dependent background noise profile (including reverberation) across the usable band of the experiment. (b) TS predictions with noise added (top row) and without noise added (bottom row) based on the three-dimensional DWBA numerical model. The solid line is the mean of the measured or added noise. The gray or white area between the two dashed lines indicates the range between \pm 1 standard deviation from the mean. The brackets indicate regions where the effect of noise addition is more prominent. Model predictions below the noise threshold were omitted. 


\subsection{Data-model comparison}

This section discusses the results of data-model comparison for the angular variation of the CPO, TS, and TS averaged over a wide range of angles of orientation. As noted earlier, unless otherwise specified, the fins were digitally removed from all the squid shapes used in the three-dimensional DWBA numerical model. The discussion of data-model comparison is based primarily on the measurements from one representative individual (0822a), but results are also shown for the other two individuals (0814a and $0819 \mathrm{c}$ ) to emphasize the consistency in the observed backscattering features.

\subsubsection{Time domain compressed pulse output (CPO) charac- teristics}

\section{CPO at normal incidence}

The temporal scattering pattern for both the experimental data and model predictions at normal incidence are compared (Fig. 2-8). Two distinct peaks were observed in the CPO envelopes of all experimental data and model predictions. For individual 0822a, the separations between the two peaks in the model predictions appear to be greater than those in the experimental data. For the experimental data, the separation translates into a spatial distance ranging between $1.74 \mathrm{~cm}$ and $2.45 \mathrm{~cm}$. For the model predictions, the corresponding distance ranges from $2.92 \mathrm{~cm}$ for the analytical DWBA prolate spheroid model, to $2.96 \mathrm{~cm}$ and $3.04 \mathrm{~cm}$ for the three-dimensional DWBA numerical model using the arms-folded and arms-splayed squid shapes, respectively. The assumed fluid-like scattering property of squid is consistent with the presence of these two dominant peaks in both the experimental data and model outputs. The separation differences between the experimental data and model predictions may be explained by the errors in modeling the actual width of the squid using dimensional measurements performed on dead specimens. The separation differences among the model predictions, on the other hand, are likely induced by the interaction of sidelobes and internal inhomogeneities in the squid body (see Sec. 2.6.4). 


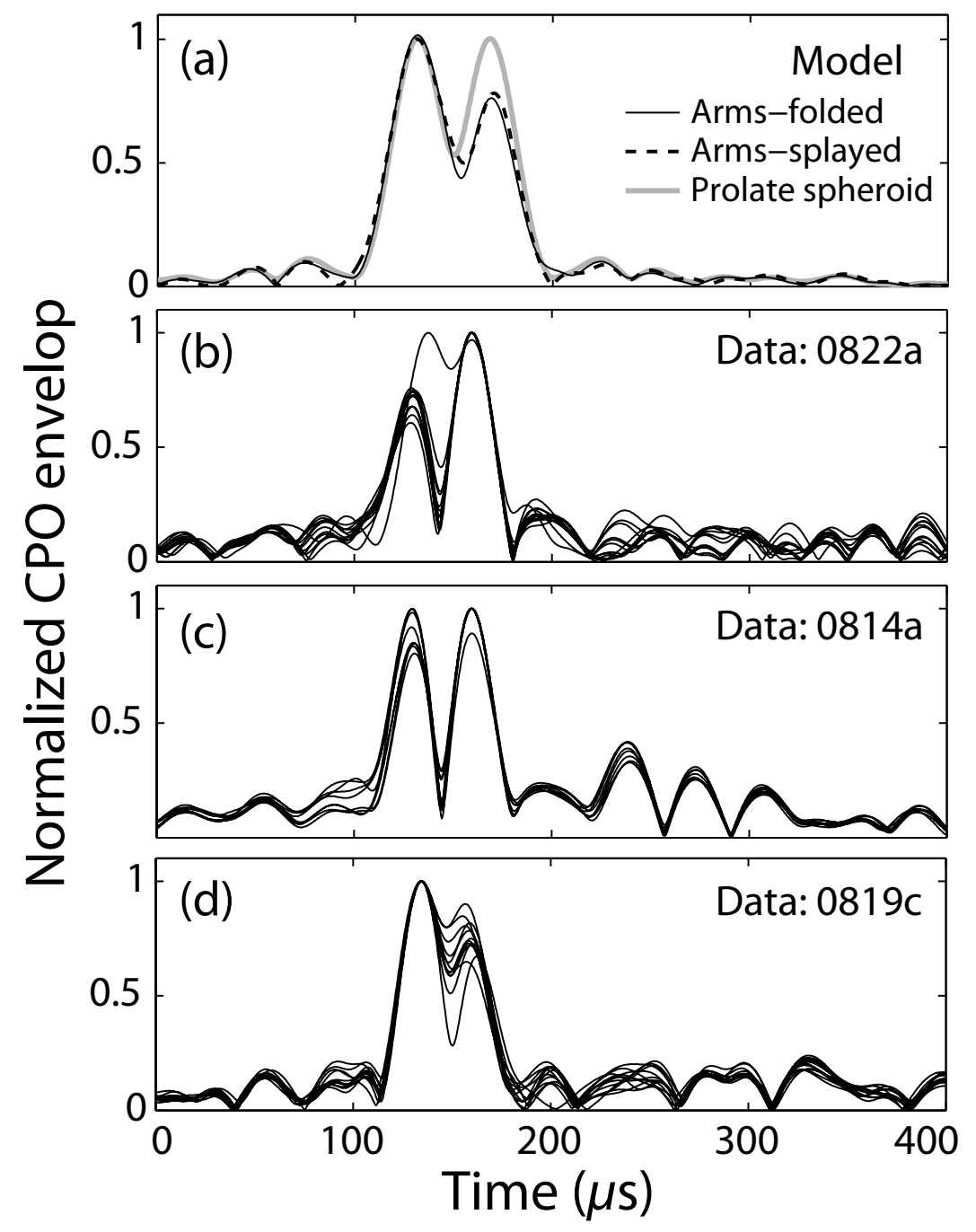

Figure 2-8: Temporal characteristics of the scattering at normal incidence. (a) Model predictions given by the three-dimensional DWBA numerical model with arms-folded and arms-splayed squid shapes and the analytical DWBA prolate spheroid model. (bd) Experimental data from three individuals, each with 15 individual pings overlaid at normal incidence. All compressed pulse output envelopes (model prediction and data) were normalized to the maximum value in each model prediction or each ping. 


\section{Angular dependence of the CPO}

There is reasonably good qualitative agreement in the general scattering pattern across all angles of orientation for the experimental data and model predictions given by the three-dimensional DWBA numerical model using an arbitrarily-chosen realization of the hybrid randomized squid shapes (Fig. 2-9). In this figure, the last of the 15 pings collected at each angle of orientation was arbitrarily chosen from the experimental data of each individual, although the results based on the other pings do not change the general pattern. The hybrid randomized squid shape was used here to model the shape of the squid during the experiment. The model shown in this figure used the hybrid randomized squid shape, which resembles the shape of the squid during the experiment, and the volume representation was scaled to match the dimension of the individual 0822a.

The sinusoidal pattern predicted in Fig. 2-6, corresponding to the location of the squid arms in the rotation, is also observed in the CPO envelopes in Fig. 2-9 for both the experimental data and the three-dimensional DWBA numerical model predictions. The model predictions also successfully capture the relative scattering strength at offnormal incidence with respect to the maximum level at normal incidence. This result, combined with the scattering characteristics at normal incidence, suggests that the DWBA-based model, which only takes into account the muscular part of the squid body, is capable of explaining a major portion of the backscattering across all angles of orientation.

\subsubsection{Angular variation of target strength (TS) at fixed fre- quencies}

The experimental data are compared to predictions given by both the analytical DWBA prolate spheroid model and the three-dimensional DWBA model using hybrid randomized squid shapes across all angles of orientation at four discrete frequencies: 60, 70, 85, $100 \mathrm{kHz}$ (Fig. 2-10, Fig. 2-11, and Fig. 2-12). These four frequencies are

chosen because they are evenly spaced across the usable band and are not in the 
(a)

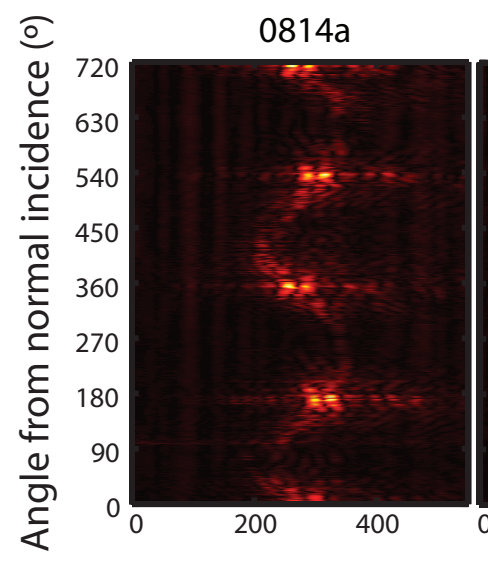

Experimental data

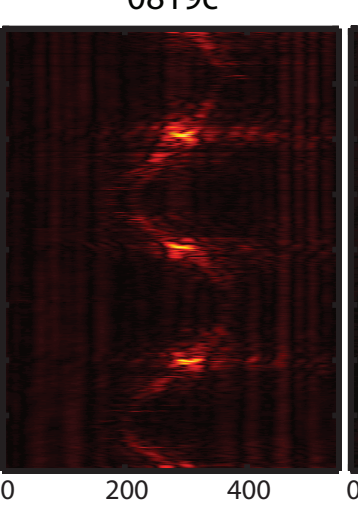

Time $(\mu \mathrm{s})$ (b) Model

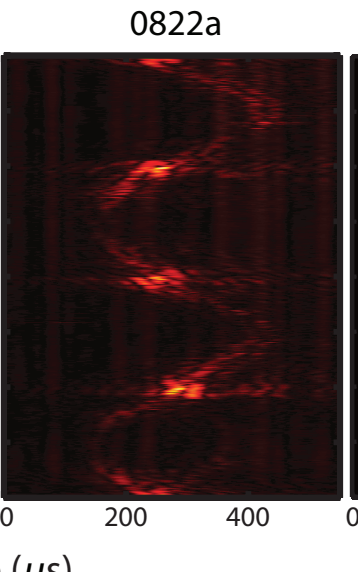

(randomized arms)

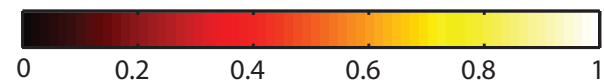

Normalized compressed pulse envelope level

Figure 2-9: Compressed pulse output envelope of (a) the experimental data from three individuals and (b) the three-dimensional DWBA numerical model using a hybrid squid shape with randomized arms over two full rotations $\left(720^{\circ}\right)$. The compressed pulse output envelopes are normalized to the maximum envelope value in each of the plot. Faint vertical lines in the experimental data are due to noise not effectively eliminated by the background reverberation subtraction. 


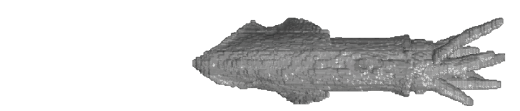

(a) Original
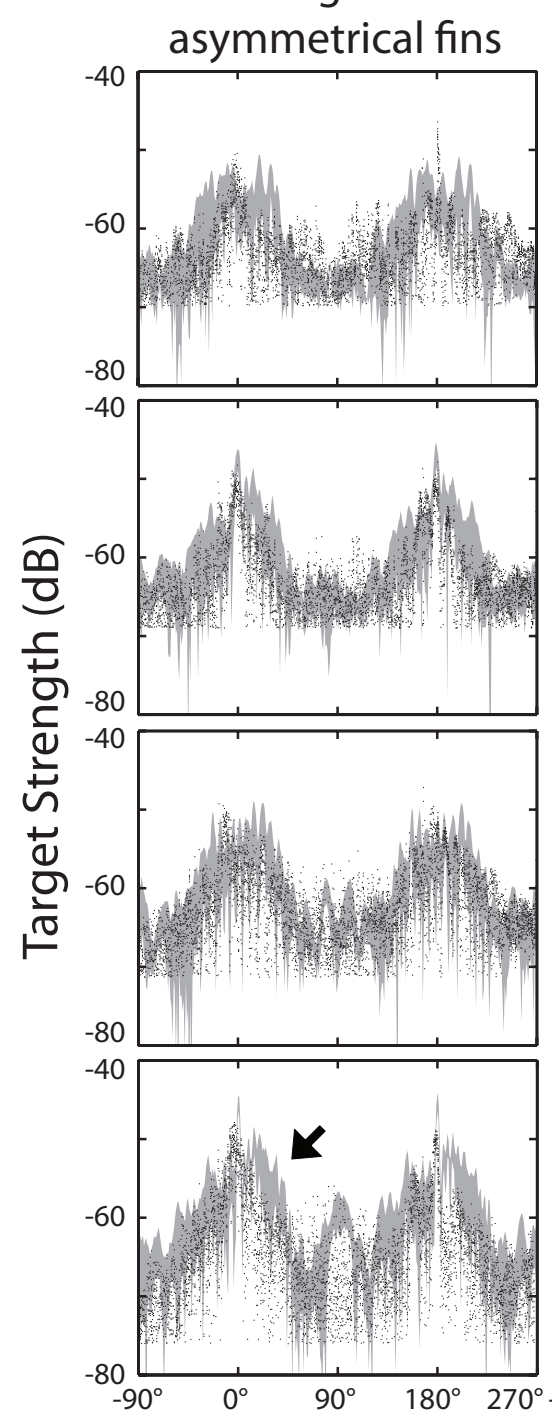

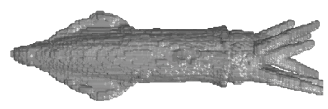

(b) Artificial symmetrical fins
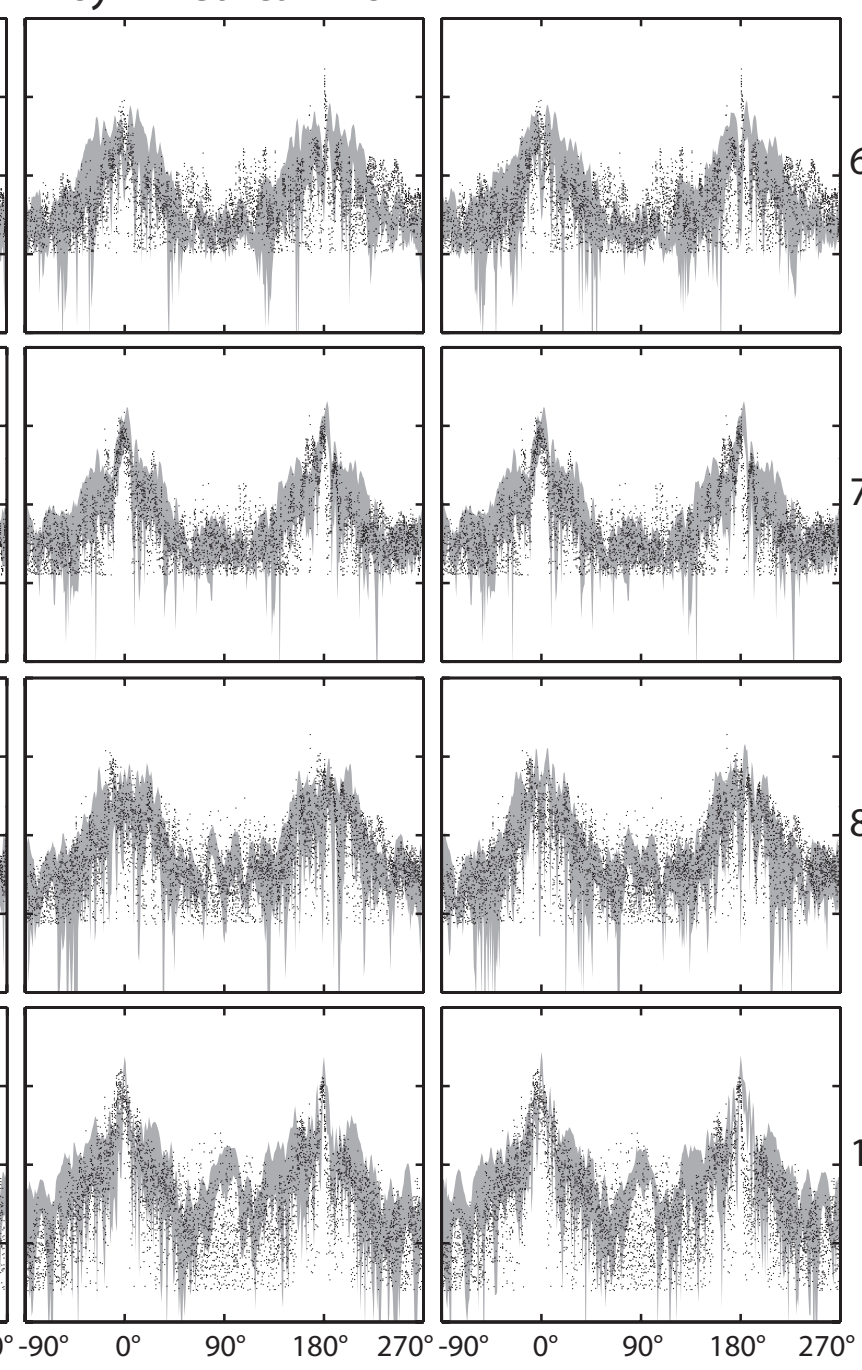

(c)
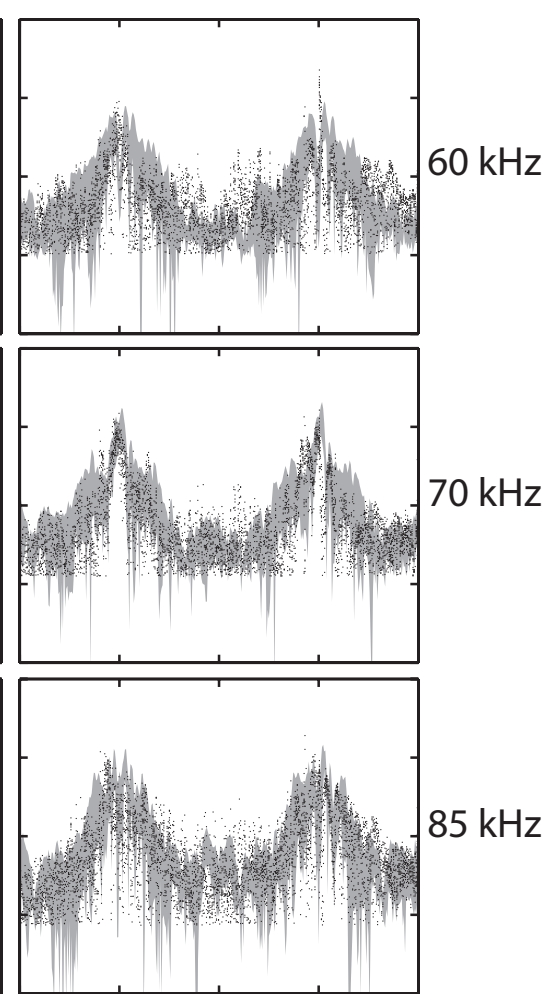

$100 \mathrm{kHz}$

Angle from normal incidence $\left({ }^{\circ}\right)$

- Experimental data $\quad$ Numerical model + noise

Figure 2-10: Data-model comparison of TS versus angle of orientation at four frequencies $(60,70,85,100 \mathrm{kHz})$ for the individual 0822a. Hybrid randomized squid shapes with three fin shapes were used in the three-dimensional DWBA numerical model: (a) original asymmetric fins, (b) artificial symmetric fins, (c) no fins. The experimental data are represented by dots. The gray area indicates the range of \pm 1 standard deviation from the mean of the model predictions. The arrow indicates the scattering contribution of the fins. The cut-off pattern near the bottom of each plot is resulted from omitting experimental data and model predictions lower than the noise threshold. 


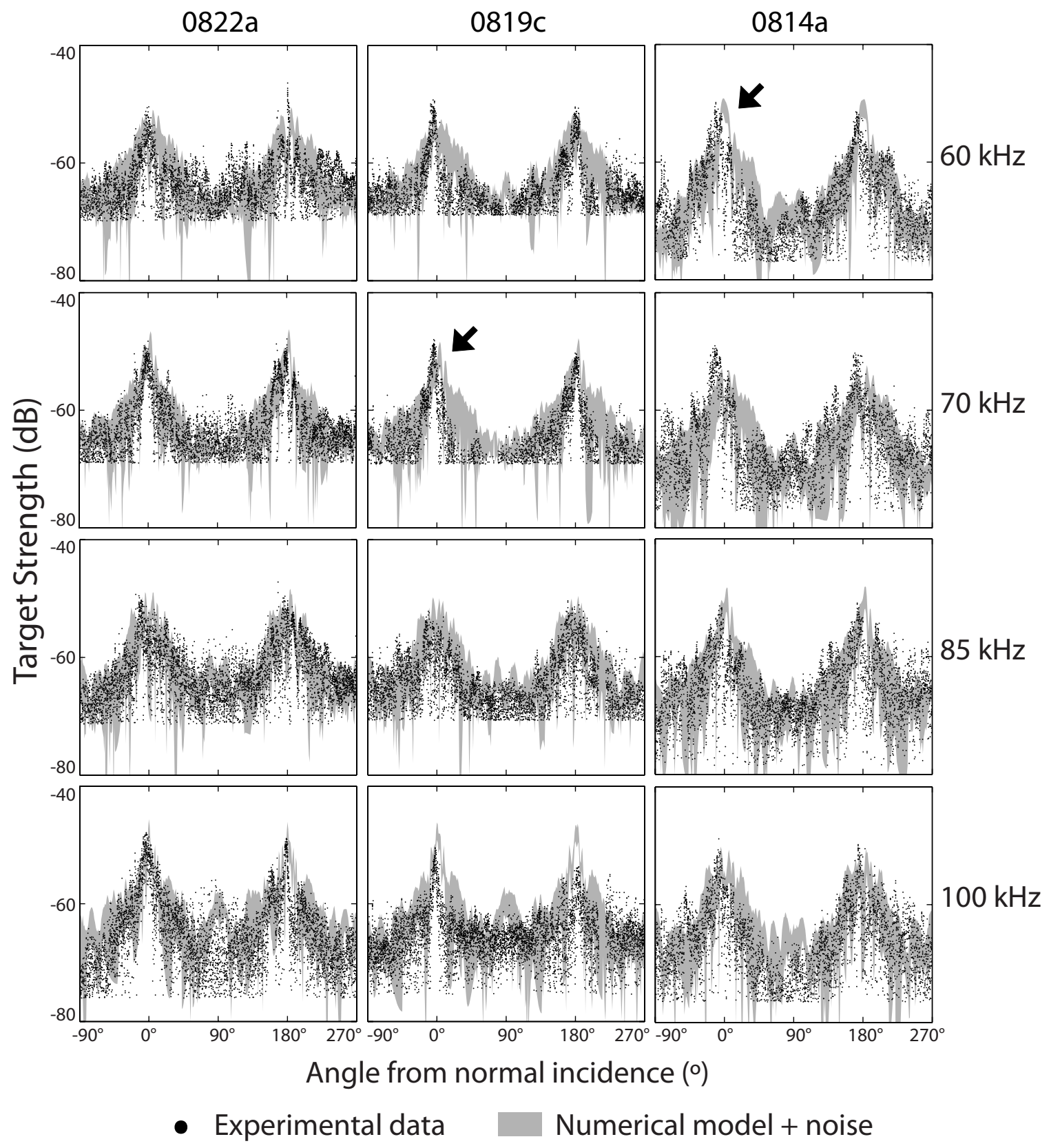

Figure 2-11: Data-model comparison of TS versus angle of orientation at four frequencies $(60,70,85,100 \mathrm{kHz})$ for three representative individuals (0822a, 0814a, 0819c). The hybrid randomized squid shapes with no fins were used in the three-dimensional DWBA numerical model, with the size of the squid shape scaled to match that of each individual. The arrows indicate the potential deviation of angles of orientation during the experiment. Other details on the figure is given in the caption of Fig. 2-10. 
high-noise band around $75 \mathrm{kHz}$ in the middle of the spectrum. Frequency-dependent noise was added to the model predictions as mentioned in Sec. 2.3.3.

In general, the predictions given by the three-dimensional DWBA numerical model agree well with the experimental data across all angles of orientation with the best correspondence at $70 \mathrm{kHz}$ and $85 \mathrm{kHz}$ (Fig. 2-10). Among the three types of mantle shapes used, the no-fins mantle (right column) shows the best agreement with the experimental data. This may be associated with the resting posture of the squid during the experiment, in which the fins hung downward and wrapped against the mantle and resulted in a shape that is similar to the no-fins mantle. The shape of the fins has a pronounced effect on the scattering pattern at the "shoulders" near normal incidence (around $20^{\circ}-40^{\circ}$ from normal incidence, as indicated by the arrow in Fig. 210). This effect is the most prominent on one side of normal incidence in the model predictions using the asymmetric-fin mantle, and less prominent and more symmetric for the model predictions using the symmetric-fin mantle. No prominent shoulders are observed in the experimental data at any frequencies for all three individuals.

The analytical DWBA prolate spheroid model, on the other hand, significantly underestimate the TS at angles roughly $>30^{\circ}$ on both sides of normal incidence, even with the same amount of noise added into the model as in the case of the threedimensional DWBA numerical model (Fig. 2-12). This result is consistent with the results in Jones et al. (2009).

\subsubsection{Frequency dependence of TS at near normal incidence}

The spectra of experimental data are compared to predictions given by both the analytical DWBA prolate spheroid model and the three-dimensional DWBA model using several squid shapes at near normal incidence (Fig. 2-13). Both the experimental data and model predictions were averaged between $\pm 2^{\circ}$ from normal incidence. The experimental data were summarized by the mean and standard deviation of the TS measurement. Due to the observed potential deviation from normal incidence in the cases of individual 0814a and 0819c (indicated by the arrows in Fig. 2-11), averages were also performed for data with their angles of orientation adjusted by $\theta_{a d j}$. Results 


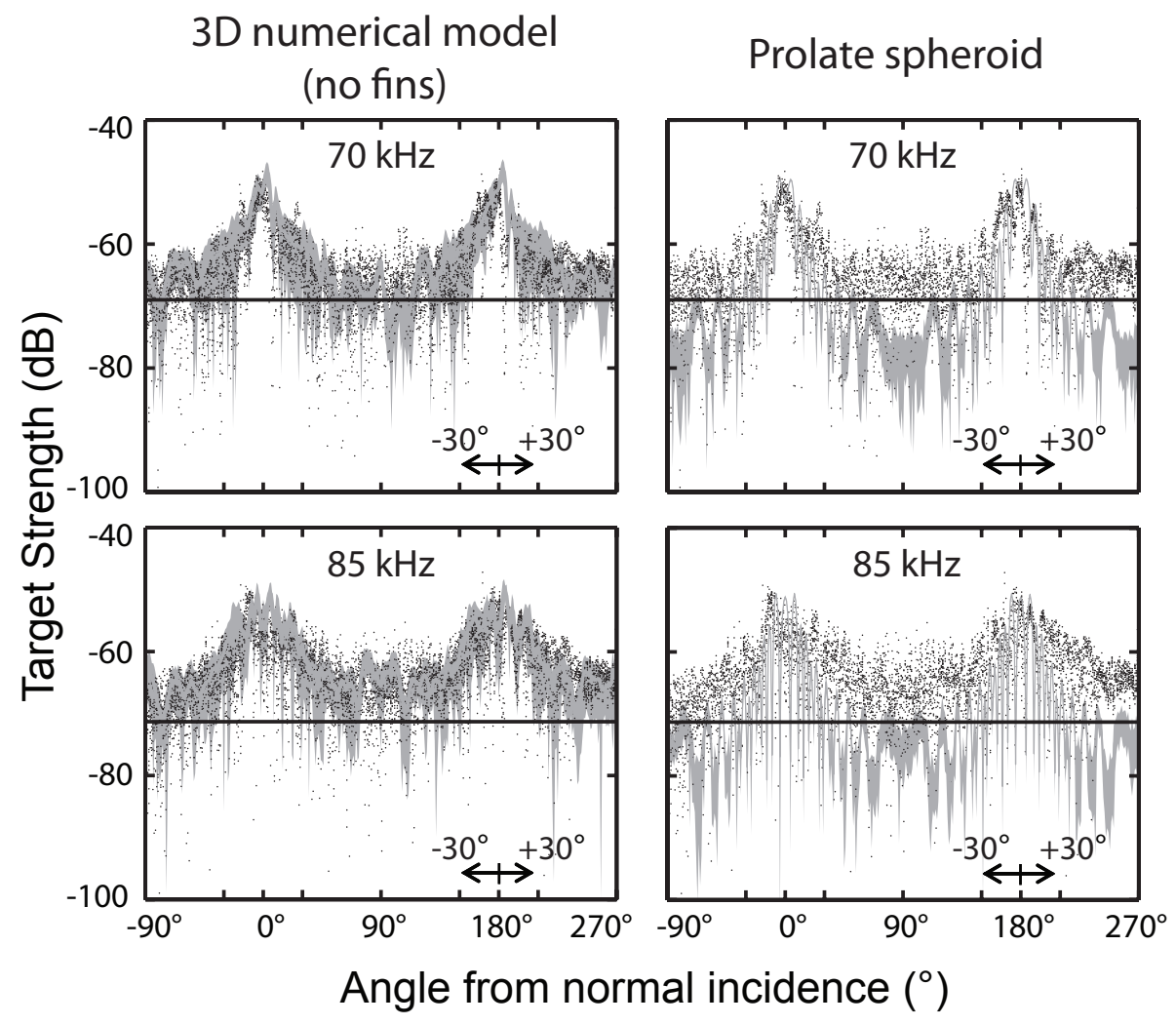

- Experimental data

- Noise threshold

Left: Numerical model + noise

Right: Prolate spheroid model + noise

Figure 2-12: Comparison of the performance of the three-dimensional DWBA numerical model and the analytical DWBA prolate spheroid model at two frequencies Frequency-dependent noise was added to both models to enable valid comparison with the data. Dots represent the ping-by-ping experimental data for the individual 0822a. The gray area indicates the range of \pm 1 standard deviation from the mean of the models. Note that the experimental data and model predictions lower than the background noise threshold (black lines) were not omitted in here to illustrate the difference clearly. 

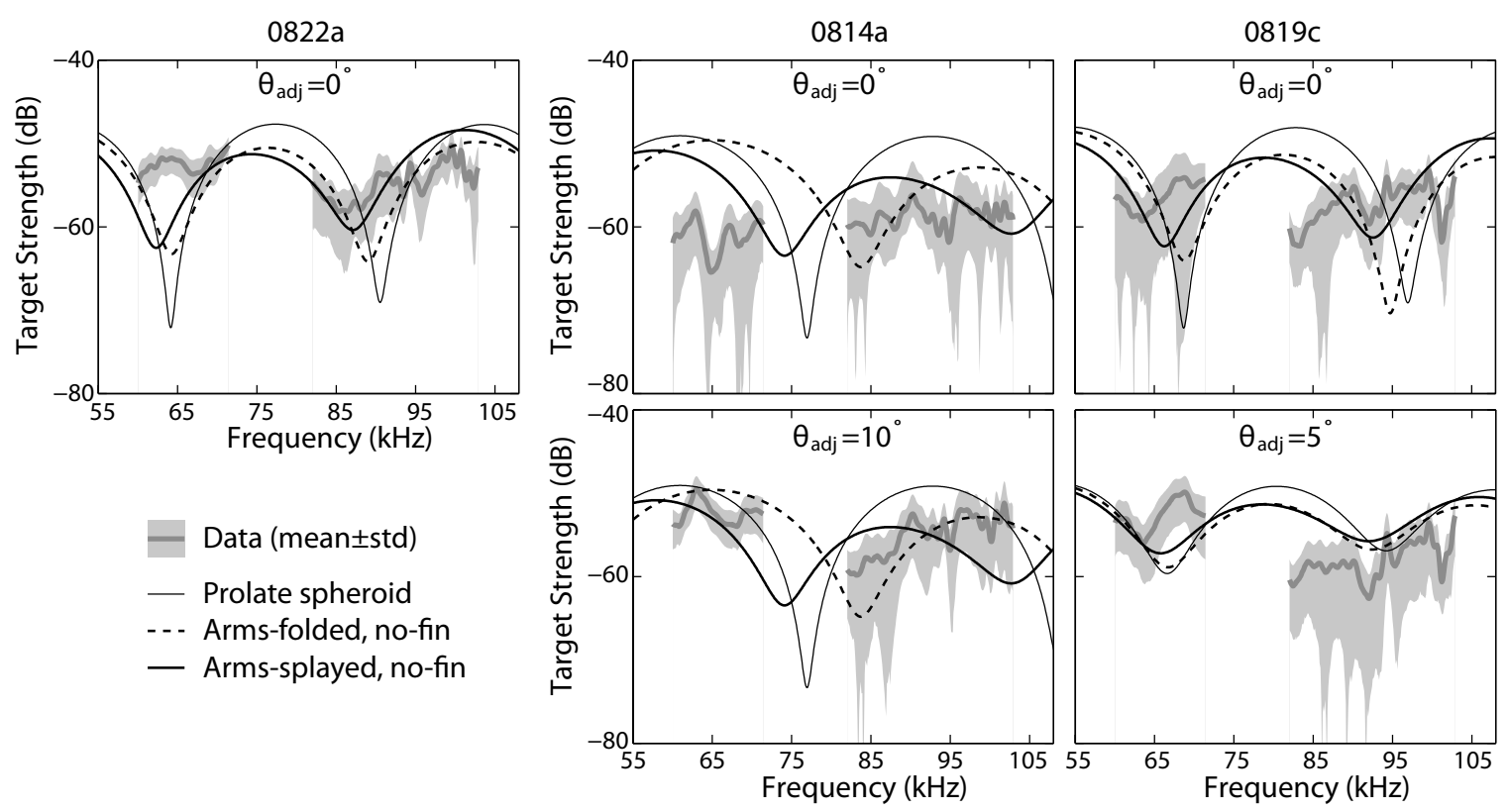

Figure 2-13: Data-model comparison of TS spectra averaged between $\pm 2^{\circ}$. The angle of orientation of experimental data were adjusted by $\theta_{a d j}$ in cases where potential deviation in the angles of orientation were observed (see Fig. 2-11).

are shown for TS averages produced both with and without such adjustment. The dimension of the prolate spheroid and the squid shapes used in the three-dimensional DWBA numerical model were scaled according to the dimension of each individual squid.

In all cases, the measured TS varies significantly across frequency with a standard deviation between 5 to $20 \mathrm{~dB}$. Although the measured TS was broadly consistent with TS predictions given by the models, the prominent spectral structure resulting from the interference of the backscattering from the front and back interface of the model shapes are not observed in the experimental data. These results suggest that the although the contribution from the weakly-scattering muscles can account for the majority of backscattering energy (Fig. 2-8 and Fig. 2-9), influence from other potential scattering sources within the squid body may be responsible for the complex spectral characteristics of TS measurements, since the exact structure of the TS spectra is sensitive to the interaction of all contributing sources of scattering (Stanton et al., 1998a). 


\subsubsection{TS averaged over angle-of-orientation distribution}

To assess the model performance under possible field conditions, averaged TS predictions in both the dorsal-ventral and lateral planes given by the analytical DWBA prolate spheroid model and the three-dimensional DWBA numerical models are compared in Fig. 2-14. Experimental data, which are only available in the lateral plane, were also averaged and compared to the model predictions [Fig. 2-14(b) and Fig. 215]. At each angle, experimental data from all pings were used in the average. The averages were obtained assuming the angles of orientation are normally-distributed with a mean angle $\mu$ and a standard deviation $\sigma$. The calculations were limited to within \pm 2 standard deviations from the mean. Averages were performed on the differential backscattering cross sections $\sigma_{b s}$ and converted to TS. Fig. 2-14 shows TS, in contrast to the reduced TS (RTS) shown in Fig. 10 in Jones et al. (2009). Since the choice of the normalizing length factor (mantle length or total length of the squid) affects the RTS values, all comparisons here were done based on TS.

The dorsal-ventral plane TS averages are relevant to data collected by downwardlooking sonars for fisheries applications. A previously reported angle-of-orientation distribution for free-swimming squid $\left([\mu, \sigma]=\left[-4^{\circ}, 11.1^{\circ}\right]\right)$ was used (Arnaya et al., 1989). TS predictions were also averaged over three other angle-of-orientation distributions with off-normal mean angles $\left(\mu=-20^{\circ},-40^{\circ},-60^{\circ}\right)$ and identical standard deviation $\left(\sigma=10^{\circ}\right)$. The angle of orientation was defined as a negative value when the arms were placed under the horizontal axis [see examples in Kang et al. (2005)].

In the dorsal-ventral plane, the averaged TS predictions given by the threedimensional DWBA numerical model are generally higher than the predictions given by the analytical DWBA prolate spheroid model for most of the frequencies [Fig. 214(a)]. The differences between these two models are larger when the angle-oforientation distributions are dominated by off-normal angles. Although no experimental data were available to assess the model performance, the analytical DWBA prolate spheroid model is likely to under-predict the actual TS averages, as suggested by the results of the data-model comparison in the lateral plane (see below) and the 
(a) Dorsal-ventral plane

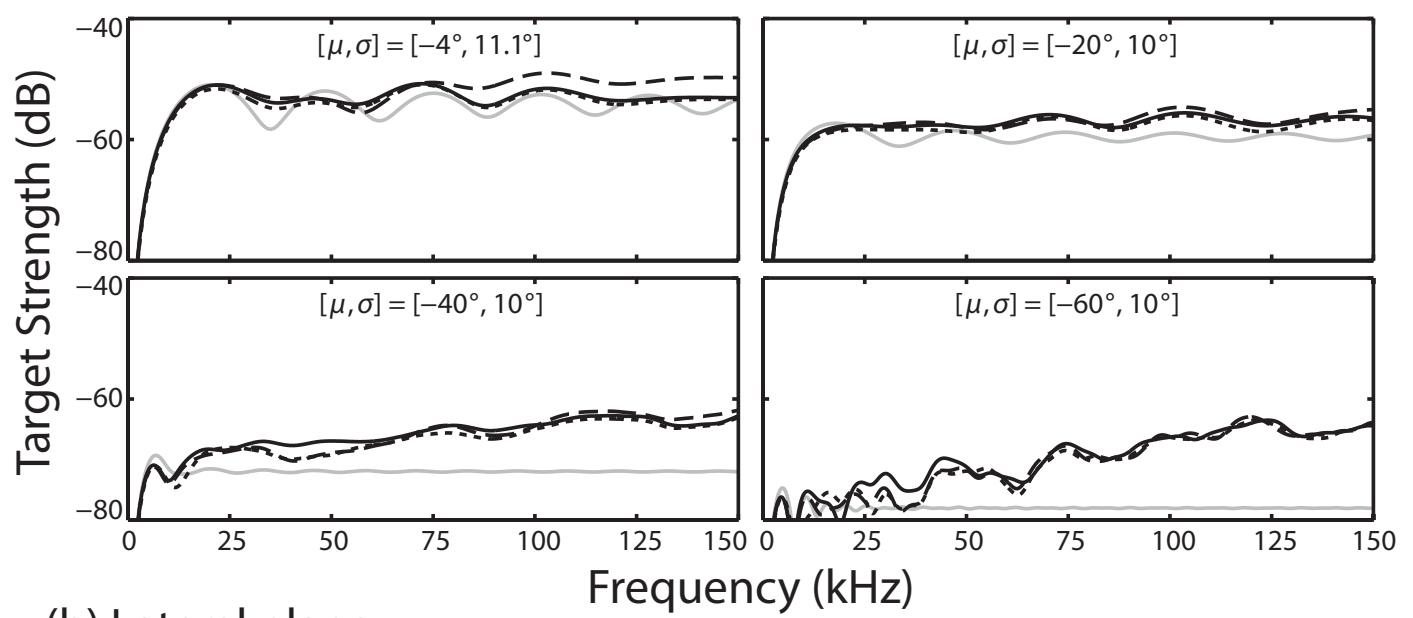

(b) Lateral plane

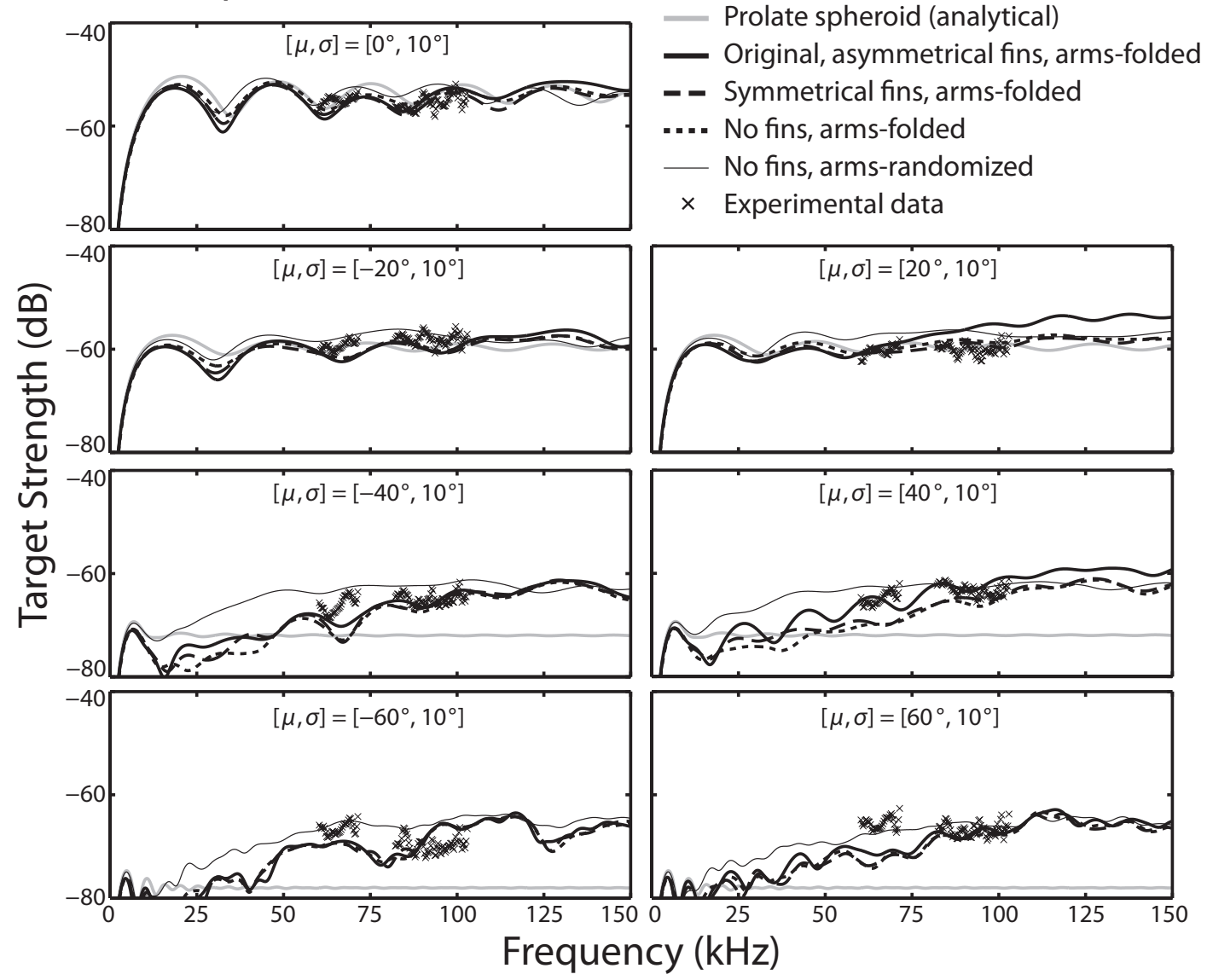

Figure 2-14: Averaged TS versus frequency for the experimental data, the analytical DWBA prolate spheroid model, and the three-dimensional DWBA numerical model using both fixed and hybrid randomized squid shapes in two planes (data only available in the lateral plane). All averages were done in the linear domain over \pm 2 standard deviations $(\sigma)$ from the mean angle $(\mu)$ and converted to TS. (a) Averages in the dorsal-ventral plane. (b) Averages in the lateral plane. 


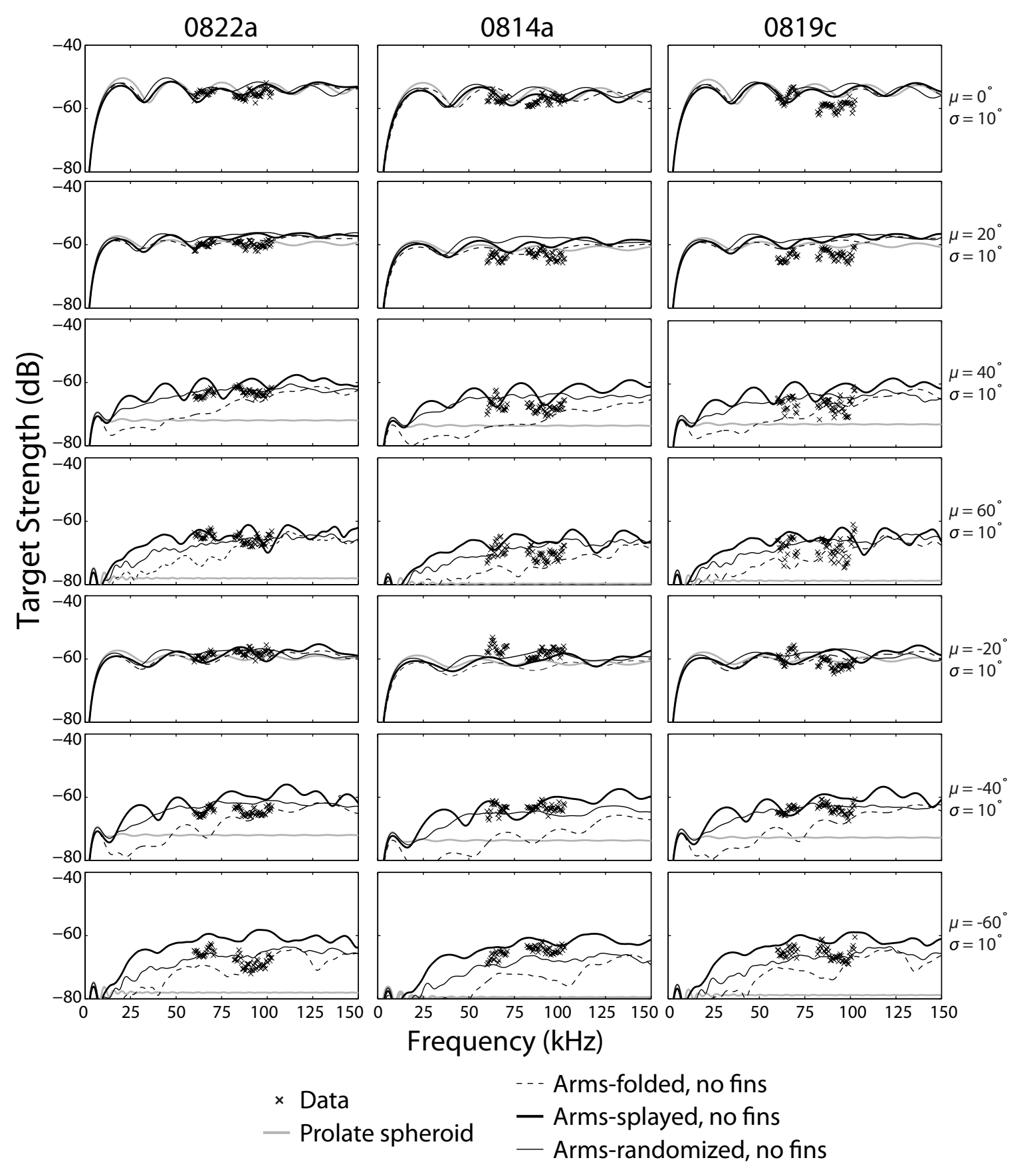

Figure 2-15: Averaged TS versus frequency for the experimental data, the analytical DWBA prolate spheroid model, and the three-dimensional DWBA numerical model using both fixed and hybrid randomized squid shapes in the lateral plane. Results from three individual squid are shown. Only a subset of models from those used in Fig. 2-14 are plotted in this figure for clarity and to facilitate inter-individual comparison. 
conclusions in Jones et al. (2009). The three-dimensional DWBA numerical model predictions using different squid shapes remain close to one another across the frequencies, except for in the $>75 \mathrm{kHz}$ region when the symmetric-fins mantle is used. This elevation in the TS is likely due to the constructive interference produced by the horizontally-extended symmetrical fins that are perpendicular to the incident wave in this geometry.

The TS averages in the lateral plane are relevant to data collected by sonars looking near horizontally such as the outer beams of multibeam sonar systems. TS predictions were averaged over several angle-of-orientation distributions with different mean values $\left(\mu=0^{\circ}, \pm 20^{\circ}, \pm 40^{\circ}, \pm 60^{\circ}\right)$ and the same standard deviation $\left(\sigma=10^{\circ}\right)$. The angle of orientation follows the definition in Fig. 2-1(b).

In the lateral plane, when the angle-of-orientation distribution is dominated by near-normal angles $\left(\mu=0^{\circ}\right)$, all model predictions gave similar spectral structures and averaged TS values, and their performance cannot be distinguished by the experimental data (Fig. 2-14(b) and Fig. 2-15). The differences among the model predictions become larger as the mean angle deviates from normal. The three-dimensional DWBA numerical model using hybrid squid shapes with splayed or randomized arms generally generates higher TS averages compared to the predictions made using other squid shapes with folded arms. TS averages predicted using the three arms-folded squid shapes are similar, except for the elevated values in the $>80 \mathrm{kHz}$ region of $\mu=40^{\circ}$ and $\mu=20^{\circ}$ cases when the squid shape with the original asymmetrical fins was used [Fig. 2-14(b)]. These elevated values are likely produced by the specific fin orientation with respect to the sonar, as discussed in Sec. 2.3.3 and Sec. 2.4.2.

Although the three-dimensional DWBA numerical model predictions were not able to fully reproduce the experimental data across the usable frequency band for all angle-of-orientation distributions, the predictions given by different squid shapes appear to collectively bound the experimental data, except for a subset of the data near the end-on incidence $\left(\mu= \pm 60^{\circ}\right)$ for individual $0822 \mathrm{a}, \mu=-20^{\circ}$ case for individual 0814a, and $\mu=0^{\circ}$ for individual 0819c. This observation reflects the importance of knowing the squid shape accurately when predicting the TS, as well as the complexity 
of the scattering process, especially for off-normal angles of orientation.

In the lateral plane, the analytical DWBA prolate spheroid model predictions drop much more rapidly compared to the predictions of the numerical model when the angle of orientation deviates from normal incidence [Fig. 2-14(b) and Fig. 2-15)]. This rapid drop leads to the generally lower averaged TS when the angle-of-orientation distributions are dominated by off-normal angles. The analytical DWBA prolate spheroid model underestimated the TS averages in the $\mu= \pm 40^{\circ}$ and $\mu= \pm 60^{\circ}$ cases. In the $\mu=20^{\circ}$ case of individual $0822 \mathrm{a}$, this model appears to correspond well with the experimental data. However, the distribution of the predicted TS values of the prolate spheroid model in these cases were not consistent with the distribution of the experimental data, and the correspondence was merely a coincidence.

\subsection{Statistics of echoes from individual squid}

The statistics of echoes from squid were examined at four different frequencies $(60,70$, 85, and $100 \mathrm{kHz}$ ) for squid individuals 0814a, 0819c, and 0822a (Fig. 2-16 to Fig. 224). These frequencies were chosen for consistency with the investigation of echo features in the temporal and spectral domains discussed earlier in Sec. 2.4. Since all of these frequencies are in the geometric scattering region, broadly similar echo statistics features are expected. This prediction has been confirmed in the experimental results (see the sections below).

The echo pdfs were obtained using kernel density estimation (KDE, Scott, 1992) on the log-transformed echo amplitude samples collected within the designated range of angles of orientation. The samples were normalized with respect to the rootmean-square (rms) echo amplitude of all pooled samples as described in Appendix A. The samples were log-transformed prior to KDE to avoid violating the boundary condition that all echo amplitudes are positive and to produce smooth estimation in the high-amplitude tail where the number of samples are small. An inverse logarithmic transformation was used to obtain the final echo pdf in the linear domain. The KDE algorithm proposed by Botev et al. (2010) is used here for its efficiency and adaptive 
kernel bandwidth selection capability.

Note that all echo amplitude samples, including those with insufficient SNR (below the noise threshold which is defined at $6 \mathrm{~dB}$ above the background reverberation reference, see Sec. 2.2.5), were pooled for the estimation of the echo pdf. This was done in order to avoid problems in normalization caused by omitting data below a certain threshold. Furthermore, only data from the first full rotation were used due to the increased noise level during the second rotation (vertical stripes on Fig. 2-9). The angle of normal incidence in this section is defined at $180^{\circ}$, instead of $0^{\circ}$ (Fig. 21). Since all echoes were collected starting at $-10^{\circ}$, this adjustment is necessary so that data from either side of normal incidence can be included for ranges of angles of orientation wider than $\left[-10^{\circ}, 10^{\circ}\right]$.

The echo pdfs in this section are plotted in both linear and logarithmic scales in some cases, and only in logarithmic scales in others. This is done to facilitate the interpretation of echo pdfs, since histograms plotted in linear scales often convey more intuitive information regarding the distribution of the data, whereas echo pdfs plotted in logarithmic scales emphasize the tails of the pdfs.

\subsubsection{Statistics of echoes from near or at normal incidence}

Squid echoes collected within the range of $\left[75^{\circ}, 105^{\circ}\right]$ (within $\pm 15^{\circ}$ from normal incidence) were pooled to form the echo pdf at near normal angles of orientation (Fig. 216). The resultant echo pdfs contain strong Rayleigh-like features for all individuals, except for several cases at 70 and $100 \mathrm{kHz}$ where there are small deviations from the Rayleigh distribution. This result is not consistent with the expectation of highly non-Rayleigh echo pdf features result from the coherent summation of two random phasors of similar amplitudes, as suggested by the observation of the dominant echoes from the front and back interfaces between the squid and the sea water at normal incidence (Fig. 2-8).

One possible explanation to this strong Rayleigh-like features is the potential "smearing" effect produced by the contamination of echoes collected at angles fur-

ther away from the normal incidence. For example, at angles $>\left|10^{\circ}\right|$ from normal 

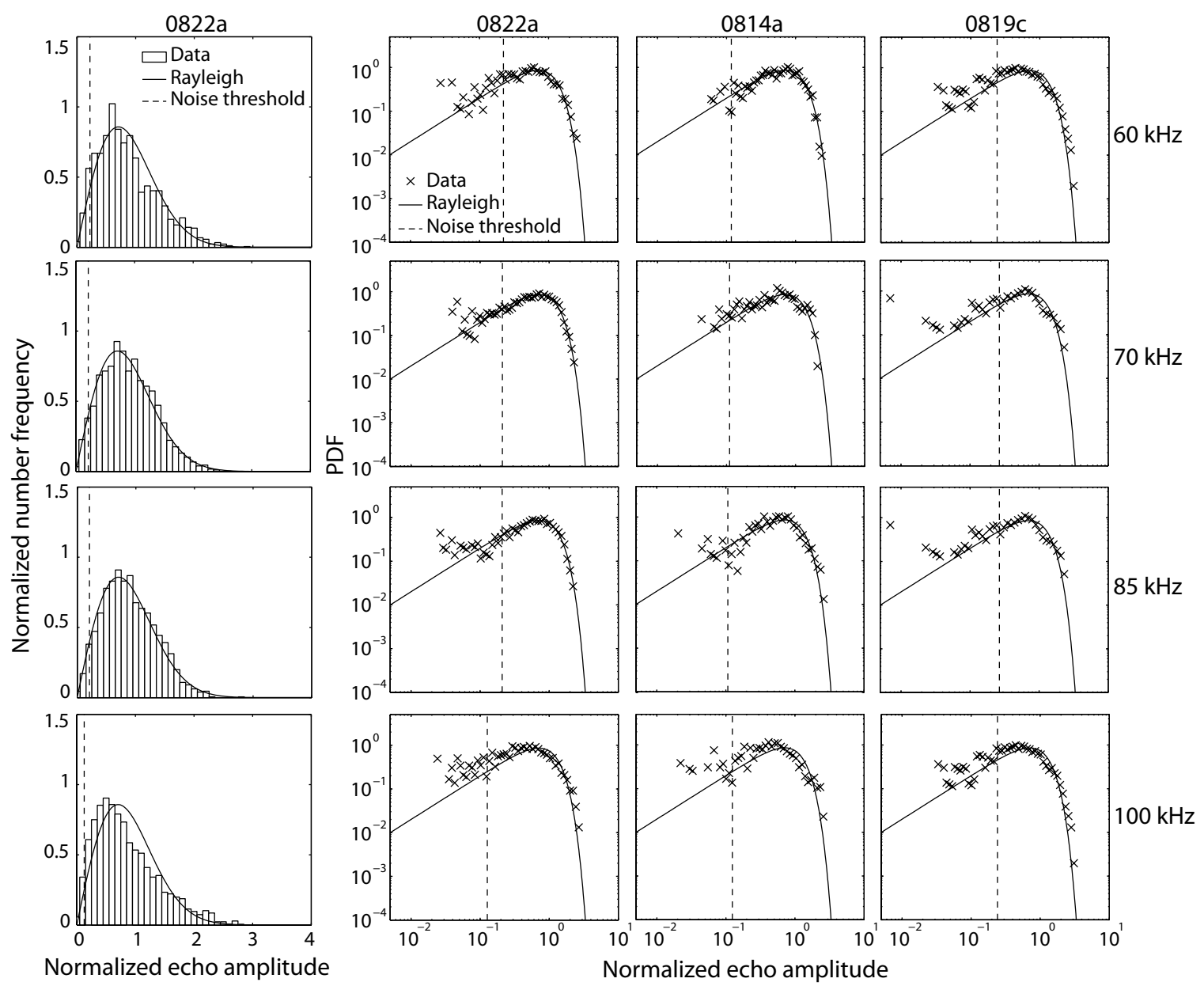

Figure 2-16: Echo pdf of data collected within the range $\left[75^{\circ}, 105^{\circ}\right]$ (within $\pm 15^{\circ}$ from normal incidence). The background noise thresholds are plotted for reference since all data were pooled to avoid problems in the normalization of echo pdf (see text). The figures in the left-most column contain identical information as those in the second column (data from individual 0822a) and are included to facilitate the comparison between results plotted in linear-linear and log-log axes. 
incidence, the temporal domain CPO structures are generally more complicated than the double-peak structure at normal incidence, which indicate the presence of other scattering sources at these angles (Fig. 2-8 and Fig. 2-9). Another possible cause of the strong Rayleigh-like features is the potential scattering contribution from other structures in the squid body even at normal incidence. This possibility has also been suggested based on the observed complex TS spectra at near normal incidence (Fig. 213). Examples of potential scattering sources include the roughness on the complex outer shape of the squid and other internal organs.

In order to clarify this question, additional analysis was conducted on a separate set of data collected immediately prior or after the measurements described in Sec. 2.2 for individual 0822a and 0814a. The experimental procedure and signals used were identical, except that echoes from 100's of consecutive acoustic pings were collected at only few angles of orientation. The temporal and spectral features of the echoes collected at normal incidence were investigated (Fig. 2-17 and Fig. 2-18), and the results are consistent with the observations presented earlier in this chapter (Sec. 2.4). However, different from what has been observed for the echo pdfs from data collected within a range of orientations near normal incidence (Fig. 2-16), echo pdfs derived from data collected only at normal incidence show strong non-Rayleigh features for all investigated frequencies except for at $85 \mathrm{kHz}$ for both individuals (Fig. 2-19). The shapes of the echo pdfs also show a variation from highly non-Rayleigh to more Rayleigh-like with increasing frequency.

These echo pdfs are compared with the echo pdfs from euphausiids and decapod shrimps previously reported (Stanton et al., 1998a, 2004). This comparison is based on the weakly-scattering material properties of these zooplankton animals, which are similar to the material properties of squid (Stanton et al., 1998a,b). For both zooplankton animals, the scattering contributions from the front and back interfaces have been observed in the temporal domain through the double-peak structures in the CPO envelopes [similar to those shown in Fig. 2-8; also see Fig. 7 in Stanton et al. (1998b)] and in the spectral domain as strong interference patterns (see Fig. 2 and Fig. 7 in Stanton et al., 1998a). 
$0822 \mathrm{a}$
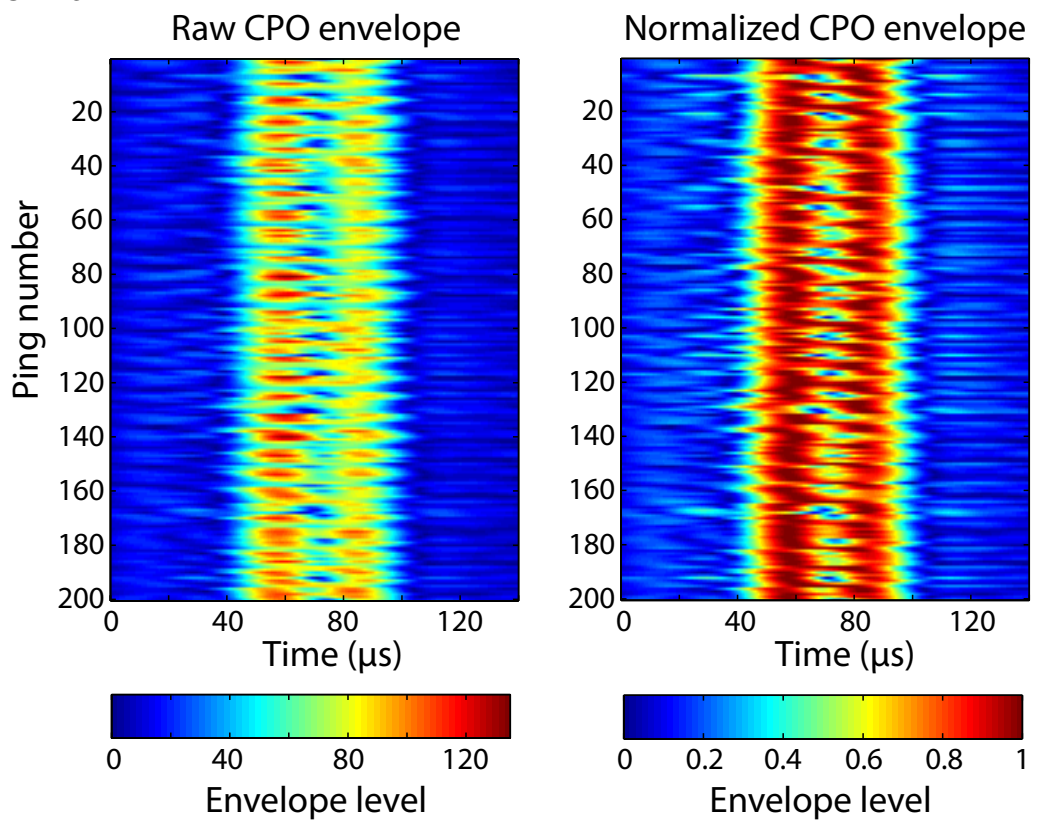

\section{$0814 a$}
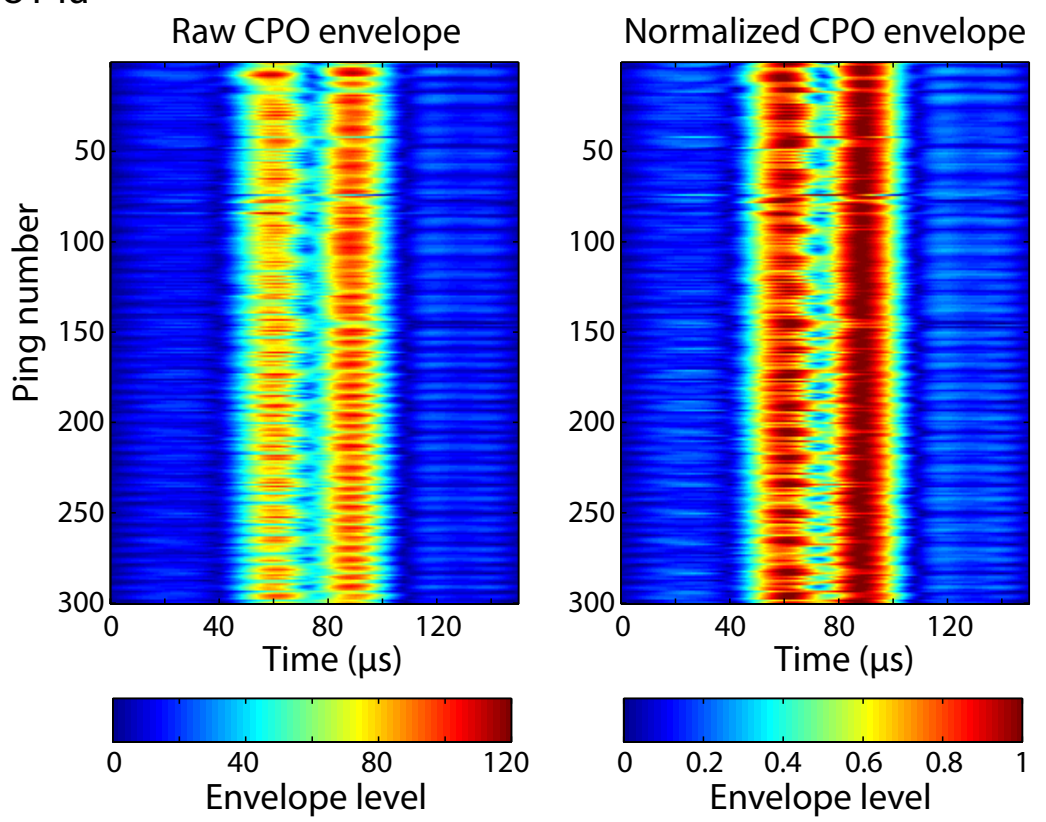

Figure 2-17: Compressed pulse output envelopes at normal incidence for individuals 0822a and 0814a. The raw compressed output envelopes on the left show the temporal variation of the magnitude of the scattering response across pings, and the normalized compressed pulse output envelopes on the right show more detail of the temporal characteristics for each ping. The compressed pulse output envelopes on the right are normalized to the maximum value in the envelope time series of each ping. 

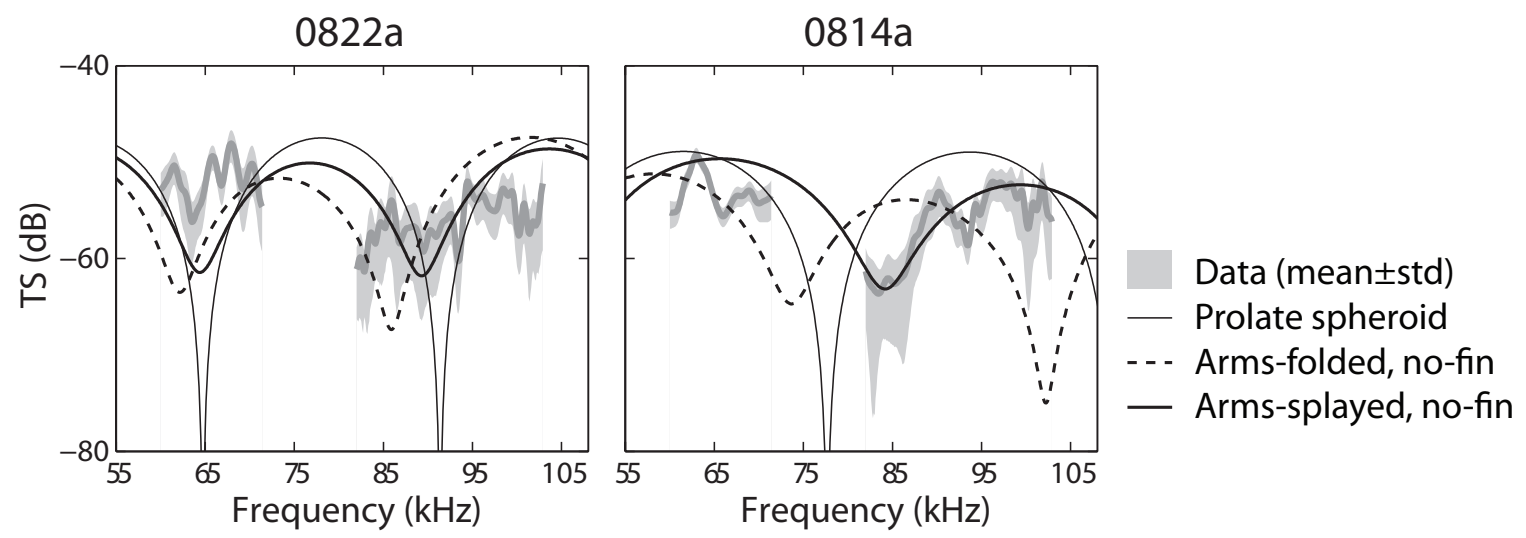

Figure 2-18: TS spectra averaged over all pings shown in Fig. 2-17. The same set of models as those used in Fig. 2-13 are shown here for reference.

The non-Rayleigh echo pdfs with narrowly-distributed echo amplitude (indicated by arrows in Fig. 2-19) suggest that the echoes contain strong coherent components, presumably from the front and back interfaces as suggested by the dominant scattering mechanisms observed at normal incidence in the temporal domain (Fig. 2-8 and Fig. 2-17). In fact, the shapes of these non-Rayleigh echo pdfs qualitatively resemble the shape of the echo pdf model results from coherent summation of two random phase sinusoids (Fig. 2 in Stanton et al., 2004), with the modes located between normalized echo amplitude values of 1 and 1.5 and the lower probability densities toward low normalized echo amplitude portions. The model assumption of random phase sinusoids is justified by the fact that the separation between the front and back interfaces of the squid body varies due to the breathing motion (contraction/expansion) of the squid mantle, which creates significant phase change across consecutive acoustic pings that can be reasonably modeled by a uniform distribution over $[0,2 \pi)$. However, the echo pdf features observed here are drastically different from the more uniformly-distributed echo amplitudes shown for a euphausiid in Fig. 4 of Stanton et al. (2004).

The variation from highly non-Rayleigh to Rayleigh-like echo pdfs with increasing frequency observed here may be intuitively explained by potential scattering processes involved at different frequencies. Specifically, the scattering from other body parts in addition to the front and back interfaces, such as the roughness on the outer shape of 
$0822 a$
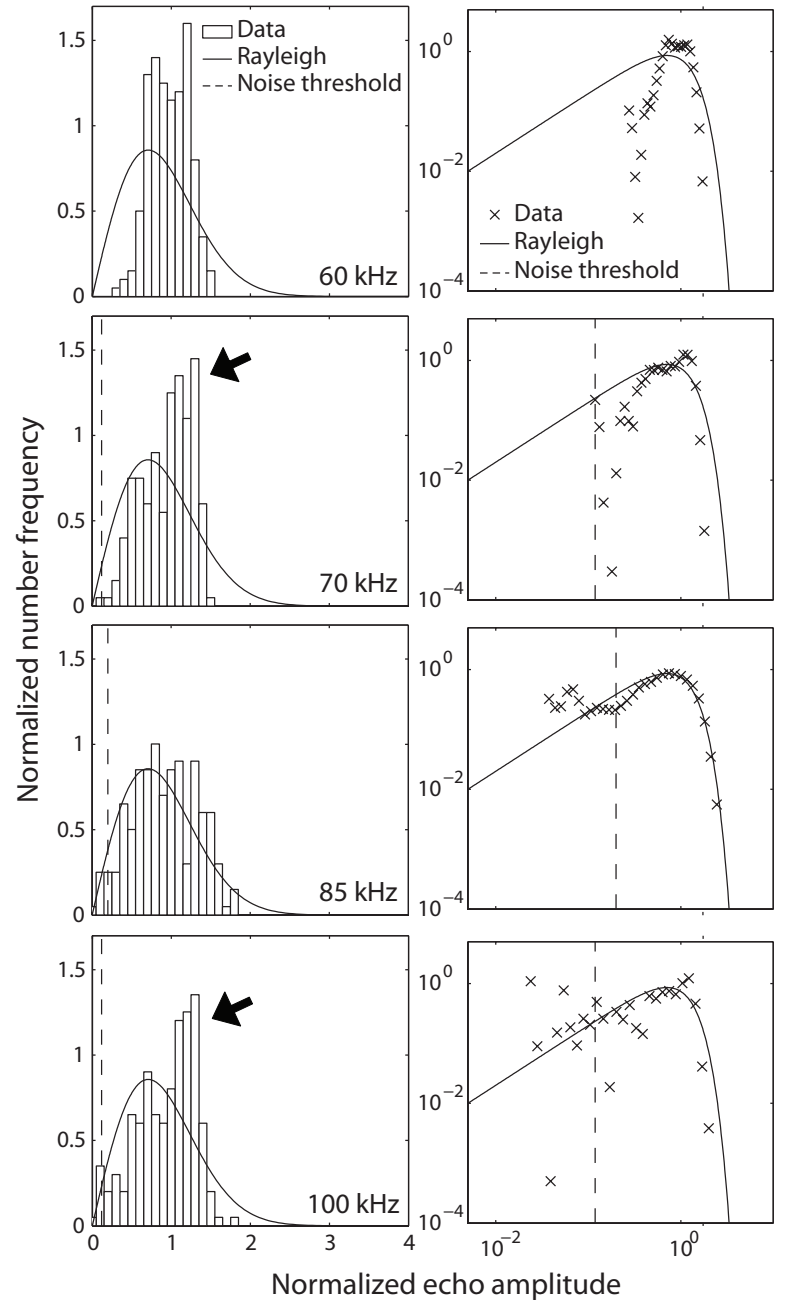

$0814 a$
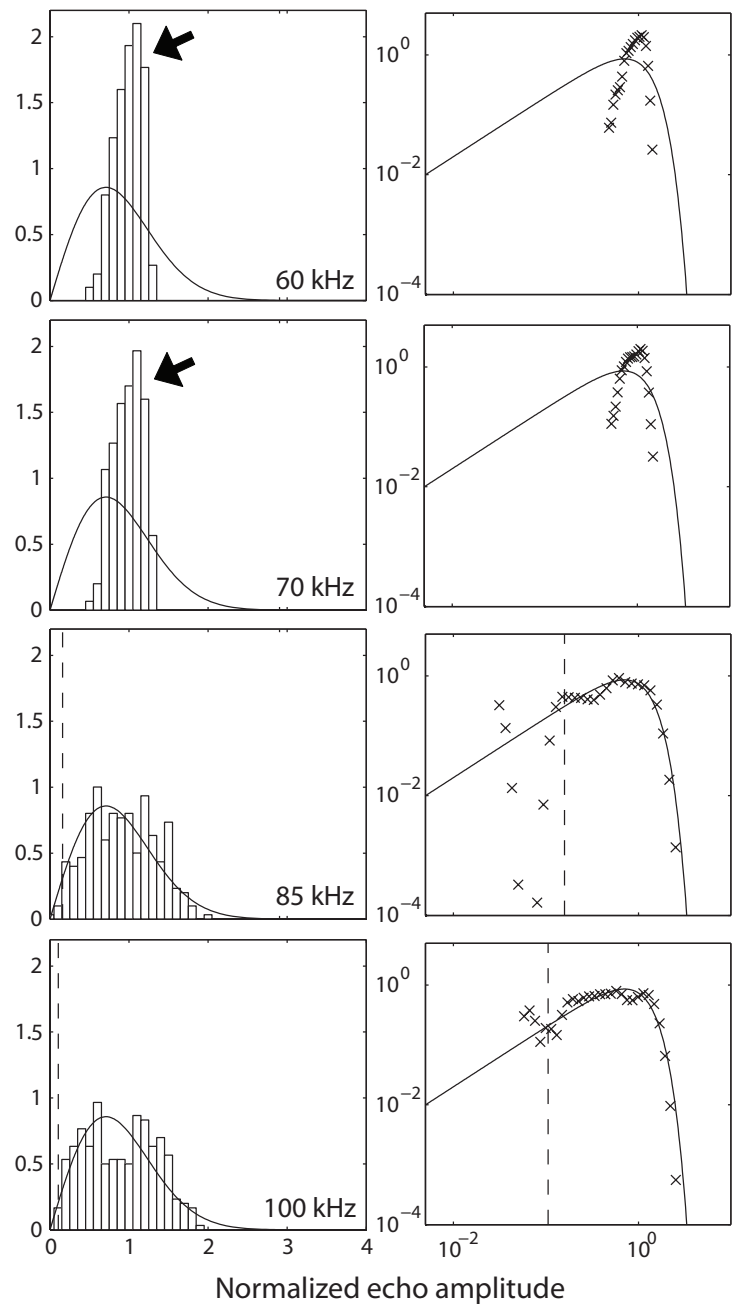

Figure 2-19: Echo pdf of data collected at normal incidence only for individuals 0822a and 0814a. Arrows indicate the narrowly-distributed echo amplitude near the mode. Details on the figures are given in the caption to Fig. 2-16. Note that noise thresholds are not plotted in some cases when all echoes are above the threshold. 
the squid, is likely to contribute more significantly at higher frequencies with shorter wavelengths, which would result in more "rays", and thus more Rayleigh-like echo pdfs. This concept is identical to the explanation offered in Stanton et al. (1998a) for the Rayleigh-like echo pdf at end-on incidence, instead of normal incidence, for a euphausiid.

However, despite the general non-Rayleigh-to-Rayleigh variation with increasing frequency, strong Rayleigh-like echo pdfs are observed at $85 \mathrm{kHz}$ for both individuals (Fig. 2-19, third row). This may be explained by the observation of a potential spectral null near this frequency for both individuals (Fig. 2-18). Specifically, slight variations in the separation between the front and back interfaces of the squid would cause shifts in the locations of the nulls and result in significant changes in the echo amplitudes, which would likely give rise to a Rayleigh-like echo pdf. This explanation is opposite to what has been proposed for the more non-Rayleigh echo pdf observed at a frequency near a null in the TS spectrum for a decapod shrimp in Stanton et al. (2004).

The echo pdf features from individual squid at normal incidence discussed above differ in a number of aspects from the observations of similar weakly-scattering marine organisms, including euphausiids and decapod shrimps, measured at normal incidence (Stanton et al., 1998a, 2004). Further investigation is clearly needed to clarify the cause of the discrepancy and the questions raised here.

\section{Statistics of echoes from other ranges of angles of orientation}

Echo pdfs were also derived from data collected in the ranges of $\left[60^{\circ}, 120^{\circ}\right]$ (within $\pm 30^{\circ}$ from normal incidence) and $\left[30^{\circ}, 150^{\circ}\right]$ (within $\pm 60^{\circ}$ from normal incidence) (Fig. 2-20 and Fig. 2-21). The shapes of the echo pdfs from different ranges of angles of orientation are compared, and the transition from strong Rayleigh-like $\left(\left[75^{\circ}, 105^{\circ}\right]\right.$ and $\left.\left[60^{\circ}, 120^{\circ}\right]\right)$ to highly non-Rayleigh $\left(\left[30^{\circ}, 150^{\circ}\right]\right)$ was found for data from all individuals (Fig. 2-22). The echo pdf from data collected at angles in the range $\left[0^{\circ}, 60^{\circ}\right) \bigcup\left(120^{\circ}, 180^{\circ}\right]$ is also derived and compared with the echo pdf from $\left[60^{\circ}, 120^{\circ}\right]$, in order to understand the cause of the transition in the shape of the echo 

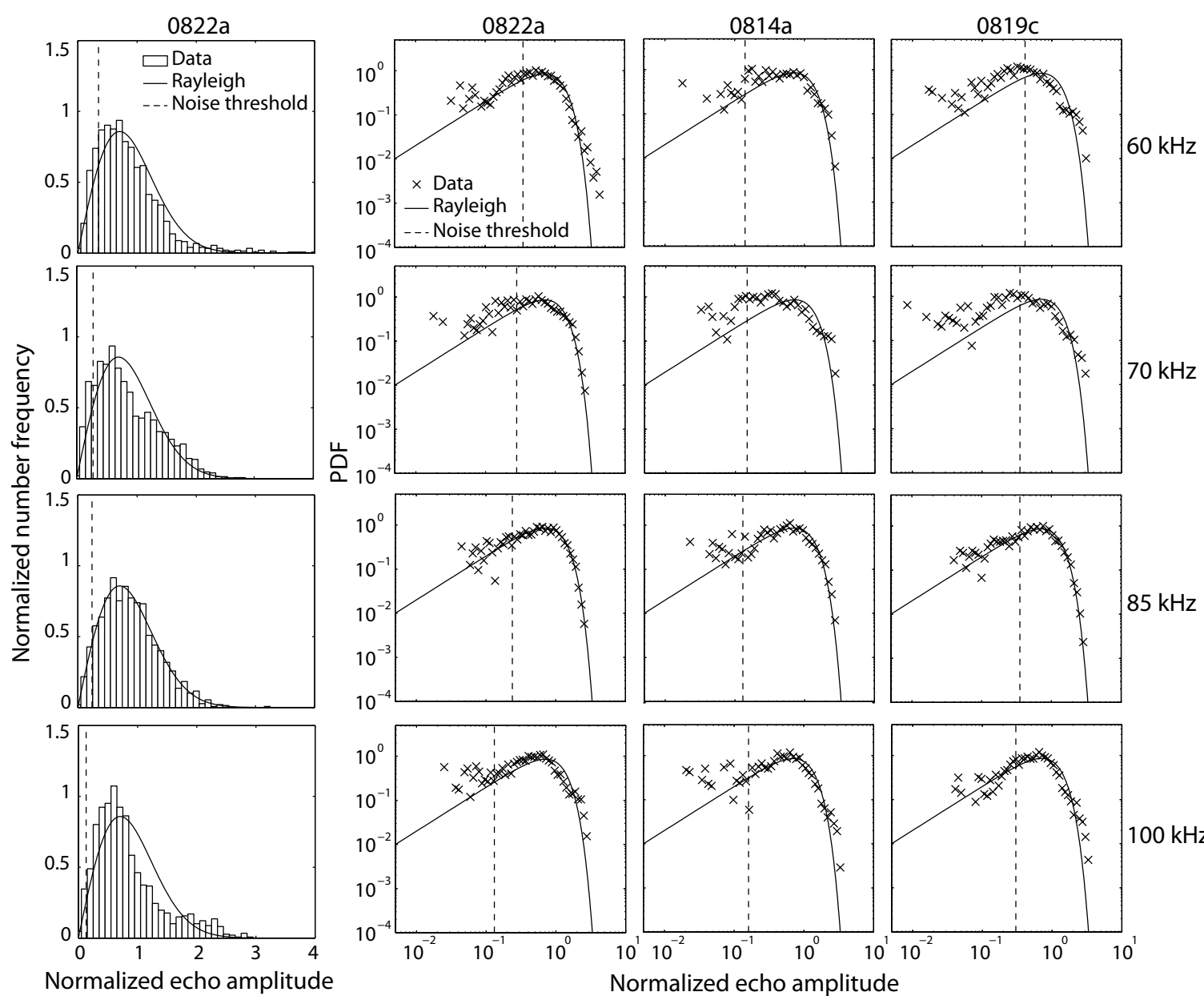

$85 \mathrm{kHz}$

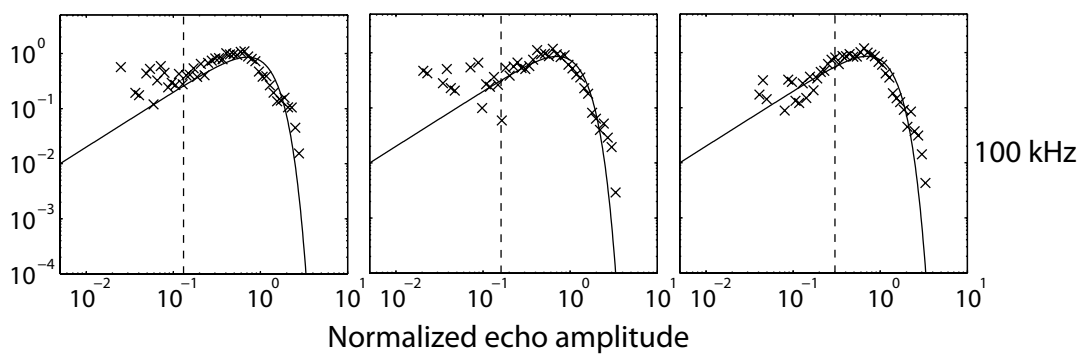

Figure 2-20: Echo pdf of data collected within the range $\left[60^{\circ}, 120^{\circ}\right]$ (within $\pm 30^{\circ}$ from normal incidence). Details on the figures are given in the caption to Fig. 2-16.

pdfs. Note that the comparison of the shapes of the echo pdfs is only possible when each echo pdf is normalized to its respective rms echo amplitude as discussed earlier in this section.

The transition from strong Rayleigh-like to non-Rayleigh echo pdfs can be explained by the sources of echoes associated with the beampattern of the scattering from squid. Specifically, with reference to the angular dependence of squid TS revealed in Fig. 2-10 and Fig. 2-11, echoes collected between $\left[60^{\circ}, 120^{\circ}\right]$ are primarily from the mainlobe of the squid scattering beampattern, whereas echoes collected from its complementary set of angles of orientation $\left(\left[0^{\circ}, 60^{\circ}\right) \cup\left(120^{\circ}, 180^{\circ}\right]\right)$ are from the sidelobes. Since strong Rayleigh-like features are observed in each of these two cases (Fig. 2-20 and Fig. 2-23), non-Rayleigh features in the echoes from [30 $\left.{ }^{\circ}, 120^{\circ}\right]$ (Fig. 2- 

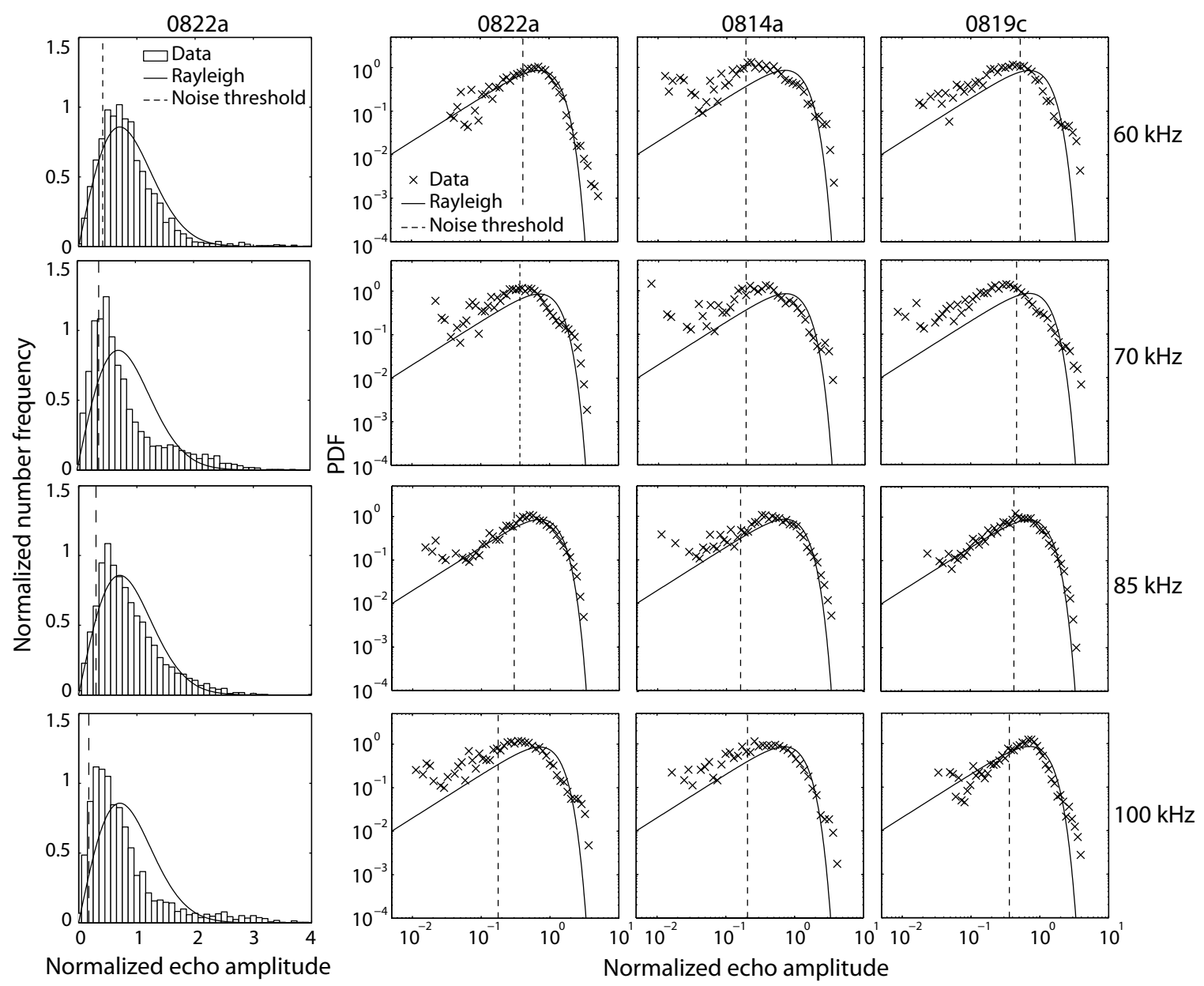

Figure 2-21: Echo pdf of data collected within the range $\left[30^{\circ}, 150^{\circ}\right]$ (within $\pm 60^{\circ}$ from normal incidence). Details on the figures are given in the caption to Fig. 2-16. 


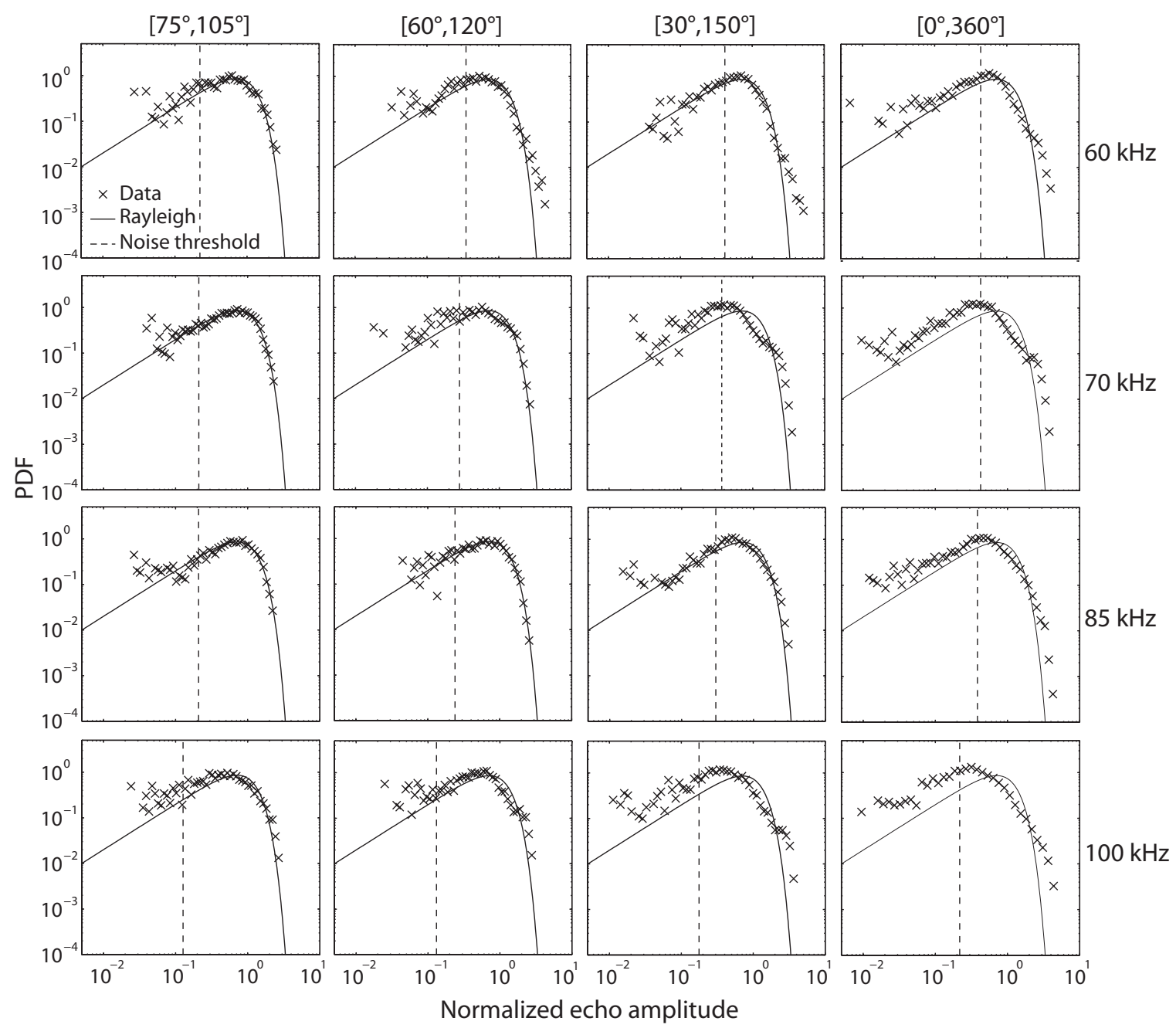

Figure 2-22: Comparison of echo pdfs of data collected within the ranges $\left[75^{\circ}, 105^{\circ}\right]$, $\left[60^{\circ}, 120^{\circ}\right],\left[30^{\circ}, 150^{\circ}\right]$, and $\left[0^{\circ}, 360^{\circ}\right]$. Details on the figures are given in the caption to Fig. 2-16. 

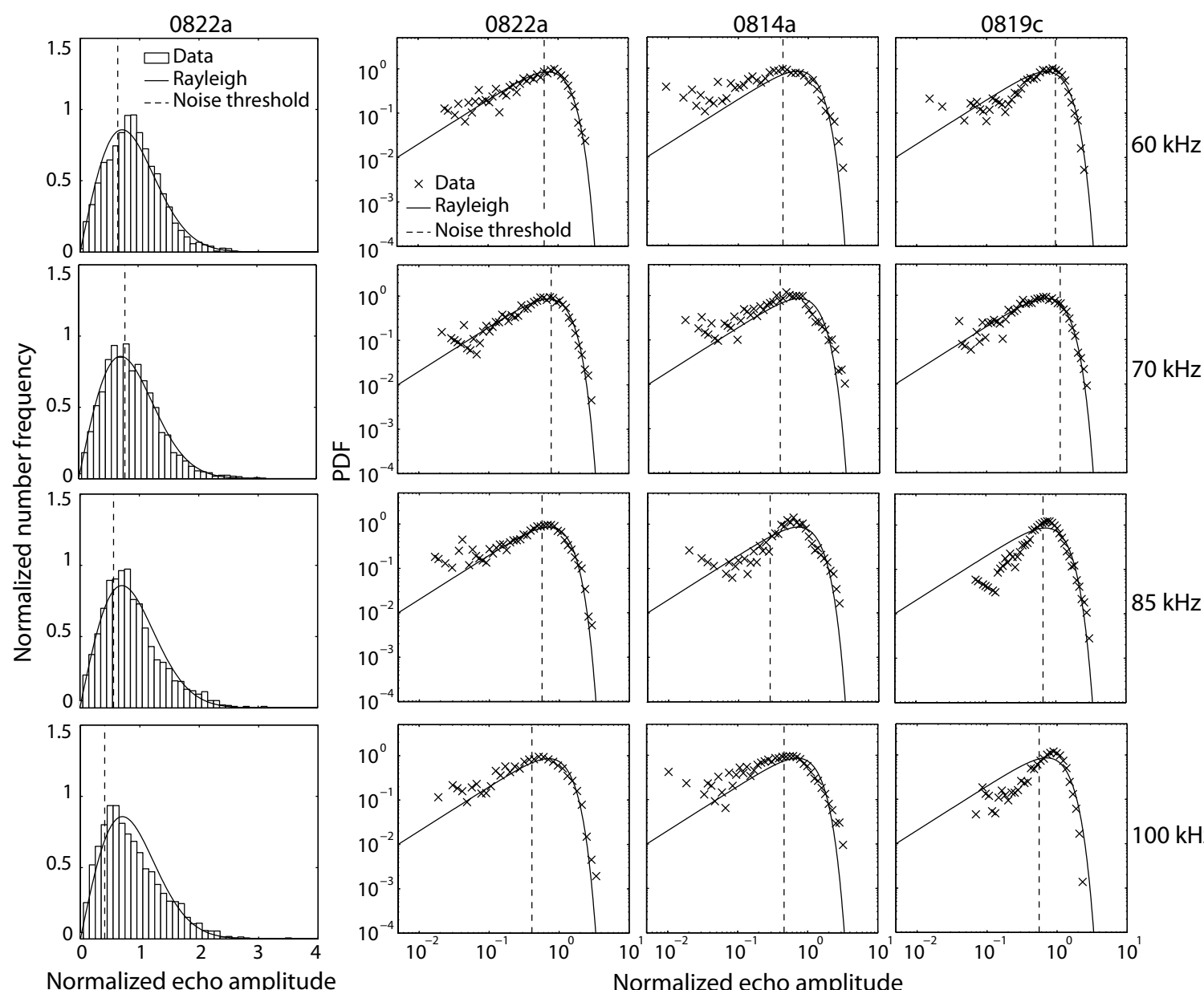

$70 \mathrm{kHz}$
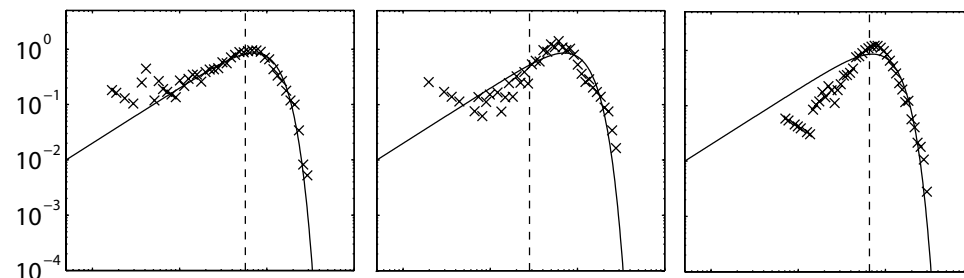

$85 \mathrm{kHz}$

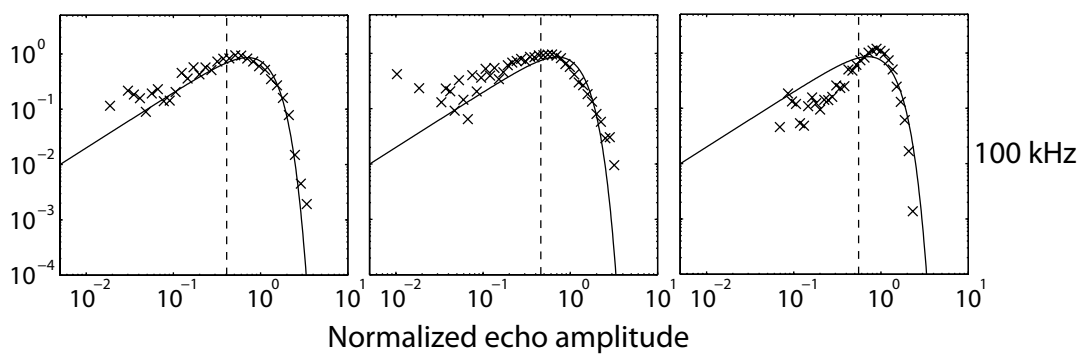

Figure 2-23: Echo pdf of data collected within the range $\left[0^{\circ}, 60^{\circ}\right) \bigcup\left(120^{\circ}, 180^{\circ}\right]$. These echoes form a complementary set of data to those shown in Fig. 2-20. Details on the figures are given in the caption to Fig. 2-16. 
21) can be viewed as a mixture of two Rayleigh-like echo pdfs with different mean values corresponding to the contributions from the mainlobe and the sidelobes. This is analogous to the "beampattern effects" discussed in Sec. 1.3.2 and Sec. 1.5, which describe the uneven modulation of the receiving echoes imposed by the transducer depending on the locations of the scatterers in the beam (Ehrenberg, 1972).

\subsubsection{Statistics of echoes from all angles of orientation}

Similar to the echo pdf from the range $\left[30^{\circ}, 150^{\circ}\right]$, the echo pdf from data collected from all angles of orientation $\left(\left[0^{\circ}, 360^{\circ}\right]\right)$ possesses highly non-Rayleigh features for all individuals (Fig. 2-24). Based on the spheroidal shape of the squid and the findings in previous sections that the smooth prolate spheroid underestimated the TS of squid at off-normal incident angles (Fig. 2-12), a Kirchhoff approximation-based theoretical model for the echo pdf from a randomly-rough prolate spheroid is used to analyze the observed echo pdf from all angles of orientation (Bhatia et al., submitted). This is done under the intuitive assumption that the roughness of the prolate spheroid can account for the observed elevated scattering from the arms and other structures of squid at off-normal incidence.

This model involves a prolate spheroid randomly-oriented in the two-dimensional plane that contains its maximum response axis, with the roughness incorporated heuristically by modulating the echo amplitude from a smooth prolate spheroid by the Rayleigh distribution in a noise-free condition (Bhatia et al., submitted). The modeling results showed that the degree to which the echo pdf deviates from the Rayleigh distribution increases with increasing degree of elongation, which can be characterized by the aspect ratio $(\epsilon)$, defined as the ratio of the length of the major axis to the length of the minor axis for a given prolate spheroid. This functional dependency of the shape of the echo pdf provides a tool for inferring the aspect ratios of volume-equivalent prolate spheroids (see Sec. 2.3.2) for squid of different sizes and shapes.

In this study, since the SNR is generally low (the mean amplitude of noise is approximately only one order of magnitude lower than the squid echoes), a numerical 

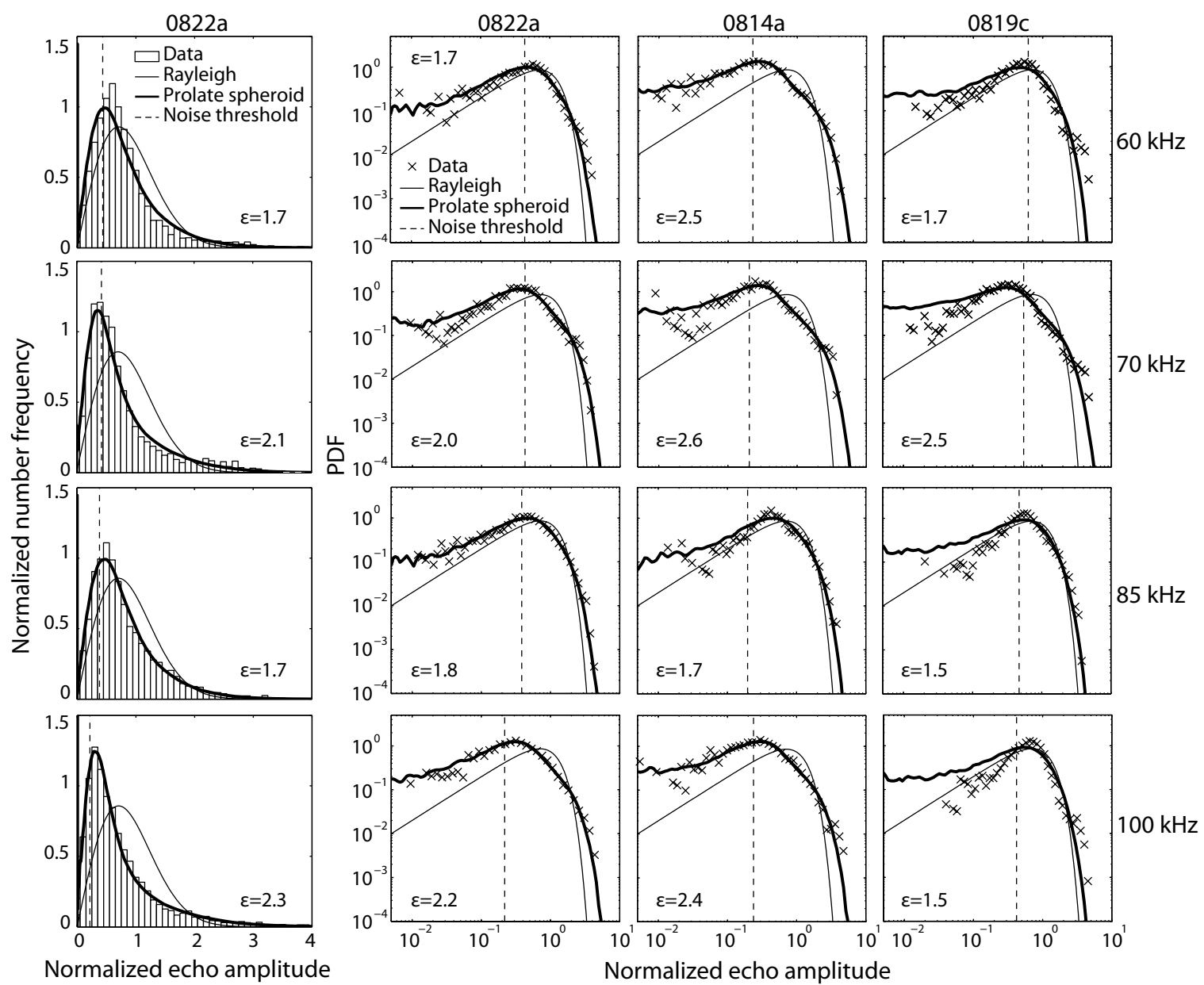

Figure 2-24: Echo pdf of data collected measured at all angles of orientation $\left(\left[0^{\circ}, 360^{\circ}\right]\right)$. The best-fitting randomly-rough prolate spheroid models are plotted with corresponding aspect ratio $(\epsilon)$ indicated. The model and fitting procedure are described in Sec. 2.5.2. Details on the figures are given in the caption to Fig. 2-16. 
approach was taken to add noise into the prolate spheroid echo pdf model through simulation. The numerical simulation follows the problem setup in Bhatia et al. (submitted) as described above. In each realization of the numerical simulation, the angle of orientation of a smooth prolate spheroid with a given aspect ratio is randomly generated by drawing a sample from a uniform distribution over $[0,2 \pi)$. The roughness is subsequently introduced by multiplying the random echo amplitude of the smooth prolate spheroid (determined by the randomly-generated angle of orientation) and a random sample drawn from the Rayleigh distribution. Random noise is then added to each of the echo amplitude samples following the procedure detailed in Appendix B. The final numerical echo pdf model was derived using simulated echo amplitude samples generated from repeated realizations by KDE.

A maximum likelihood estimator (MLE) was used to select a best-fitting aspect ratio for each echo pdf derived from the data (Azzalini, 1996). The operation of the MLE is summarized here for convenient reference. Assume $x_{1}, x_{2}, \ldots, x_{n}$ are independent and identically-distributed echo samples, this method computes the loglikelihood

$$
\begin{aligned}
l\left(\epsilon \mid x_{1}, x_{2}, \ldots, x_{n}\right) & =\ln \mathcal{L}\left(\epsilon \mid x_{1}, x_{2}, \ldots, x_{n}\right) \\
& =\ln p_{\text {model }}\left(x_{1}, x_{2}, \ldots, x_{n} \mid \epsilon\right) \\
& =\ln \prod_{i}^{n} p_{\text {model }}\left(x_{i} \mid \epsilon\right) \\
& =\sum_{i}^{n} \ln p_{\text {model }}\left(x_{i} \mid \epsilon\right),
\end{aligned}
$$

and performs the inference by maximizing the log-likelihood

$$
\hat{\epsilon}_{\mathrm{MLE}}=\underset{\epsilon}{\arg \max } l\left(\epsilon \mid x_{1}, x_{2}, \ldots, x_{n}\right) .
$$

Randomly-rough prolate spheroids with aspect ratios ranging from 1.5 to 2.6 were found to best-fit the experimental data investigated here (Fig. 2-24). However, the actual aspect ratios of volume-equivalent prolate spheroids are 6.52, 6.07, and 6.00 
for individuals $0814 \mathrm{a}, 0819 \mathrm{c}$, and 0822a, respectively. In other words, the randomlyrough prolate spheroid echo pdf model underestimates the aspect ratio of the squid. This result may be explained by the discrepancy between model assumptions and the scattering properties of the squid. Specifically, the weakly-scattering material properties, complex outer shape, and internal inhomogeneities of squid are likely to result in more "rays" compared to the impenetrable rough prolate spheroids assumed in this model. Further investigation using more sophisticated models is needed to verify this hypothesis.

\subsection{Discussion}

\subsubsection{Model performance}

Results of the data-model comparison show that the three-dimensional DWBA numerical model, which takes into account only the fluid-like soft tissue in the squid body, is capable of capturing the observed dominant scattering characteristics of squid. In particular, the presence of two dominant peaks at normal incidence in the $\mathrm{CPO}$ envelopes in the experimental data is consistent with the model predictions (Fig. 2-8), and the pattern of the time domain CPO envelopes and model TS predictions across all angles of orientation correspond reasonably well with the experimental data (Figs. 2-9 to Fig. 2-11, Fig. 2-14, and Fig. 2-15).

Results of the data-model comparison also show that the three-dimensional DWBA numerical model, compared to the analytical DWBA prolate spheroid model, is capable of giving better TS estimation once averaged over an ensemble of predictions made using a set of squid shapes with randomized arms at fixed frequencies (Sec. 2.4.2). This result is consistent with the previous conclusion reached by Jones et al. (2009) and illustrated in Fig. 9 of that paper. The three-dimensional DWBA numerical model also produces better predictions for TS averaged over a range of angles of orientation at different frequencies (Fig. 2-14 and Fig. 2-15). However, this numerical model was not able to predict the measured TS spectral curves even at angles near 
normal incidence when the backscattering is dominated by the front and back interfaces between the squid body and seawater (Fig. 2-13). This may be explained by the fact that the spectral structure of the TS is highly sensitive to the precise size, shape, orientation, and material properties, including detailed internal inhomogeneities, of the animals (Stanton et al., 1998a), as well as the scattering contribution from other sources in addition to the muscle tissue. The influence of the above parameters on acoustic scattering from squid is discussed in more detail below.

\subsubsection{Squid tissue material properties}

In this study, the soft-tissue material properties of T. pacificus, a similar species in the Pacific Ocean, have been used to model the scattering from L. pealeii. This was done under the assumption that these epipelagic squid species have similar muscle material properties, which are closely related to the habitat and ecological role of the species (Seibel et al., 2004). Different material properties will be required to predict acoustic scattering from other more distantly-related squid species, such as the larger and highly muscular jumbo squid, Dosidicus gigas, or the mid-water, ammoniacal squid with generally lower muscle density (O'Dor, 2002). In addition, variation of local material properties may be required to model the scattering from some species. For example, unlike the thickened flesh suction cups found in L. pealeii, each of the suction cups of $D$. gigas has a chitinous ring of teeth, which may have different material properties than the muscle.

\subsubsection{Scattering contribution from other potential sources}

The DWBA-based models employed in this study to predict scattering from squid only consider the fluid-like soft tissue in the squid body and do not account specifically for the scattering contribution from other body parts, such as the skull, chitinous beak, eyes, and other internal organs. The model predictions were able to reproduce the observed dominant scattering features in the time domain and give reasonable estimation of the observed TS (Figs. 2-8 to Fig. 2-15). These results show that, at 
least in the lateral plane, the majority of the scattering energy can be explained by the fluid-like scattering properties of the tissue, and the contributions from other potential body parts are relatively insignificant.

Due to constraints in tank size and the geometry of the experimental setup, there was no measurement available to directly assess the scattering contribution from the squid pen in the dorsal-ventral plane, which is of more interest to fisheries applications with downward-looking echosounders. The pen is a flat, elongated chitinous supporting structure lying internally along the length of the dorsal surface of the mantle. Therefore, if the pen is an important scattering source, its contribution is likely to lead to a large deviation between the tissue-only model predictions and the experimental data at normal incidence in the dorsal-ventral plane. However, in the study conducted previously by Jones et al. (2009), good agreement was found between the experimental data and the three-dimensional DWBA numerical model predictions for T. pacificus in the dorsal-ventral plane particularly at normal incidence. This result appears to suggest that the pen does not contribute significantly to the scattering in the dorsal-ventral plane, at least in the investigated frequency range.

\subsubsection{Squid size estimation}

One of the primary advantages of using broadband signals and pulse compression techniques is the increased spatial resolution of the measurements, which allows dominant scattering mechanisms to be determined as well as the scatterer size to be estimated. In this study, the width of the squid mantle was relatively accurately assessed by measuring the separation between the two main arrivals in the CPO envelope at normal incidence, assuming an internal sound speed. However, there were some discrepancies between the physically-measured width and the acoustically-inferred width based on the experimental data as well as model predictions for the individual 0822a (Fig. 28). There was also significant variability in the inferred width of the squid based on the experimental data for all individuals. This variability is likely a result of the expansion and contraction of the mantle during squid ventilation.

For the case of individual 0822a, the mean acoustically-inferred width from the 
experimental data is smaller than any of the model predictions. It is possible that this is a result of the error associated with performing dimensional measurements on dead specimens (obtained after the acoustic measurements were complete). In this case, the maximum mantle width measured from a collapsed mantle cavity of a dead squid is likely wider than the mantle width for the same animal when alive. Recall that the squid shapes used in the models are scaled according to the dimensional measurements of the squid.

The acoustically-inferred widths from the model predictions are also different from the actual width of the model volumes, although only by a small proportion $(<$ $2.67 \%)$. The inferred width of the analytical DWBA prolate spheroid model (2.92 $\mathrm{cm})$ is slightly smaller than the short axis of the prolate spheroid $(3 \mathrm{~cm})$. The inferred width of the three-dimensional DWBA numerical model $(2.96 \mathrm{~cm}$ using the armsfolded squid shape and $3.04 \mathrm{~cm}$ using the arms-splayed squid shape) are also different than the actual average width $(3 \mathrm{~cm})$ of the digital squid volume. The variability of the inferred widths is a combined effect of the shape of the scattering object and the shape of the auto-correlation function (width of the mainlobe and height of the sidelobes) of the replica signal used in pulse compression processing. For the analytical DWBA prolate spheroid model, the smaller inferred width is an artifact resulted from the summation of the sidelobes of the response of one of the water-body interfaces and the mainlobe of the response of the other interface. For the three-dimensional DWBA numerical models, the high degree of internal inhomogeneities in the squid volume (see Fig. 2 in Jones et al., 2009) interacts with the sidelobes of the auto-correlation function and smears the peak locations in the CPO envelope (Lee et al., 2010).

\subsubsection{Squid shape}

This study has shown that the shape of the modeled squid may also have to be adjusted to obtain the most accurate scattering predictions. In this study, the squid shape used in the three-dimensional DWBA numerical model was based on SCT images of different individuals than those used in the actual scattering measurements. Better agreement may have been achieved by using the same individuals for the scat- 
tering measurements and the SCT scans. The results of this study also show that the most accurate model predictions are achieved by using squid shapes that do not include the fins and have loosely-folded arms, most closely resembling the observed shape of the squid during the actual experiment (Fig. 2-10). In the natural environment, squid usually swim with their arms fully folded and the fins fully extended. Splayed arms are only observed during fighting, defense, or reproduction (Hanlon et al., 1999; Hanlon and Messenger, 1998). Therefore, for acoustic data collected in the field, model predictions made using squid shapes with folded arms and symmetric fins may produce the best agreement with the data.

\subsubsection{Modeling squid aggregations}

Depending on the species, squid in their natural environment may be found dispersed or in aggregations. To accurately model the scattering from squid aggregations, care must to taken to select the shape of individual squid in the aggregation, the distribution of the squid angle of orientation relative to the sonar beam, as well as the distribution of squid size. For data collected using downward-looking sonar beams, the shape of the fins is particularly important when the angle of orientation is dominated by near-normal angles [Fig. 2-14(a)]. In field applications, the three-dimensional DWBA numerical model using squid shapes with fins fully extended is likely to give the best modeling results (see Sec. 2.6.5). However, the errors in the estimates of the angle-of-orientation and size distributions may result in larger errors in the estimated biomass than the choice of different squid shapes (Lawson et al., 2006). The angle-of-orientation distribution of the squid relative to the sonar beam can also dictate the choice of models. For example, when the angle of orientation is dominated by near-normal angles, all models, including the analytical DWBA prolate spheroid model, give similar results for averaged TS. However, when the angle of orientation is dominated by off-normal angles, the three-dimensional DWBA numerical model is necessary to accurately predict averaged TS, and the analytical DWBA prolate spheroid model is likely to under-predict the averaged TS in this case. 


\subsubsection{Statistics of echoes from individual squid}

This study has shown that the echo pdf of individual squid can vary from highly nonRayleigh to Rayleigh-like depending on the range of angles of orientation included in the analysis and the acoustic frequency (Fig. 2-16, Fig. 2-20, Fig. 2-21, and Fig. 224). The analysis also showed that the shape of the echo pdf from all angles of orientation can be explained based on the scattering beampattern of the squid, and that the observed echo statistics features can be modeled using a randomly-oriented, randomly-rough prolate spheroid with an effective aspect ratio (Fig. 2-24). These results can be used to improve the parameterization of echo pdf models for individual organisms, which are useful in modeling the statistics of echoes from aggregations of organisms in Chapter 3 and Chapter 4.

However, the echo pdfs observed at normal incidence (Fig. 2-19) contain features that are not consistent with the temproal and spectral features observed at this angle (Fig. 2-8, Fig. 2-13, Fig. 2-17, and Fig. 2-18). For example, the transition from strong non-Rayleigh to Rayleigh-like echo pdfs with increasing frequency (Fig. 2-19) suggests that the scattering from different body parts other than the body interfaces of squid, or the roughness on the outer surface may be important at higher frequencies, which may explain the lack of distinct interference patterns in the TS spectra. In contrast, the resemblance between the observed non-Rayleigh echo pdf features and the modeled echo pdf produced by the summation of two random sinusoids suggests that the echoes from the front and back interfaces of squid dominates the scattering at normal incidence. Therefore, the results presented here are inconclusive and merit further investigation through future experiment and modeling analysis.

\subsection{Summary and conclusions}

This study presents the first set of controlled laboratory measurements of broadband acoustic scattering from live squid at all angles of orientation in the lateral plane. The results indicate that sophisticated models are necessary to predict the scattering over a wide range of important conditions. 
The performance of two DWBA-based models, a closed-form analytical prolate spheroid model and a three-dimensional numerical model, have been compared to the data. By using the three-dimensional DWBA numerical model and digitally manipulating the squid shape, it was possible to assess the scattering contributions from individual body parts, such as the fins and the arms. It has been found that the analytical DWBA prolate spheroid model can accurately predict the measured TS over a narrow range of angles of orientation near normal incidence, while the threedimensional DWBA numerical model can predict the measured TS across a wider range of angle of orientation. Results of the data-model comparison also show that (1) both DWBA-based models are capable of explaining the observed dominant scattering features at normal incidence, but only the three-dimensional DWBA numerical model can explain the dominant scattering features at angles of orientation well away from normal incidence, (2) the contributions from the front and back interfaces of the squid dominate the scattering at normal incidence, while the arms have a significant effect at other angles, and (3) the scattering from the squid appears to be dominated by the fluid-like weak scattering properties of squid. Statistical analysis of the data also revealed the strong non-Rayleigh features in the echoes from randomly-oriented individual squid, which provide additional information that is complementary to the above temporal, spectral, and angular characterization toward understanding the scattering mechanisms of live squid.

One of the ultimate goals of this study is to improve the acoustically-inferred estimates of the distribution and abundance of squid in the ocean. For squid species commonly found in aggregations, potential field applications for the downward-looking sonar and the side-looking sectors in the multibeam systems were investigated by comparing the measured TS to the TS predictions, both averaged over several angleof-orientation distributions. It has been found that the analytical DWBA prolate spheroid model can only predict the averaged TS for angle-of-orientation distributions dominated by near-normal angles, while the three-dimensional DWBA numerical model was able to reproduce the observed averaged TS except for several small subsets of the data. The incorporation of precise modeling parameters in the three- 
dimensional DWBA numerical model, including an accurate representation of the squid shape, squid muscle material properties, as well as appropriate estimation of the angle-of-orientation and size distributions, are required to improve the accuracy of the TS estimates. The non-Rayleigh features in the squid echoes should also be incorporated into echo statistics models of aggregations of organisms in the analysis of data collected in the field.

Finally, squids are a diverse group of animals with a wide range of sizes and shapes, but general anatomical features for these animals are similar for most species. Therefore, the understanding developed in this study through measurements and modeling of the scattering from L. pealeii may be applied to guide the modeling for other squid species. 


\section{Chapter 3}

\section{Statistics of echoes from mixed assemblages of scatterers with}

\section{different scattering amplitudes and numerical densities}

\subsection{Introduction}

The ability to accurately characterize and discriminate between the echoes from various sources of scattering is crucial to many radar and sonar systems (Le Chevalier, 2002). When a sonar/radar beam scans through a volume or surface of interest, the echoes fluctuate as a result of variation in the composition of scatterers, their orientation distribution, locations of these scatterers in the beam, and numerical density of the scatterers included in the analysis window. The statistics of the echoes provide an avenue to infer key properties of the scatterers. For example, the shapes of the probability density functions (pdfs) can be used to estimate the numerical density of the scatterers (Stanton, 1985a,b). Furthermore, understanding these pdfs is important in predicting sonar/radar performance when discriminating between a target of interest and clutter (Abraham and Lyons, 2010; Watts and Wards, 2010). 
When the resolution cell of a sonar/radar system contains a large number of identical scatterers, the central limit theorem holds for the real and imaginary components of the echoes, and the amplitudes of echo envelopes (or, more precisely, the magnitude of the complex echo) are Rayleigh-distributed (Goodman, 1985). Here, the "resolution cell" is defined as the volume subtended jointly by the mainlobe and sidelobes of the sonar/radar beam and the range resolution of the system. However, when the above conditions are not satisfied, non-Rayleigh-distributed echoes with heavy tails can occur. The "tail" is defined for the region where the values of the echo amplitude are relatively high and probability densities are relatively low. When the resolution cell contains only a small number of scatterers, the resultant echo amplitude pdf (hereafter referred to as the echo pdf) is highly non-Rayleigh, owing significantly to the random weighting factors due to random locations of the scatterers in the beam. The distribution associated with this echo amplitude modulation is termed the "beampattern pdf" (Chu and Stanton, 2010; Ehrenberg, 1972) and this (beampattern) effect is one of the primary factors for non-Rayleigh-distributed echoes.

Another common reason for non-Rayleigh-distributed echoes to occur is when there is more than one type of scatterer in the aggregation included in the analysis window (where the window is much larger than the resolution cell of the system, resulting in many statistically independent samples within the window). In this case, the statistics can be non-Rayleigh even when the total number of scatterers is large. Here, the same "type" of scatterers refer to scatterers with the same scattering amplitude (or, if it is a random variable, the same amplitude distribution with the same mean amplitude) at the frequency under consideration. For example, there could be a single strong scatterer interspersed within a large number of scatterers of another type, which has a much smaller scattering amplitude. Without the strong scatterer, the echoes would be Rayleigh-distributed due to the relatively weak scatterers. However, if the echo from the strong scatterer is large enough so that it is comparable to or larger than the collective echo from the weak scatterers, its presence can significantly influence the statistics, increasing the tail and making it non-Rayleigh. This type of condition is prevalent in nature such as in the cases of the presence of occasional large 
fish foraging in a zooplankton patch (Onsrud et al., 2004), sparsely distributed rock outcrops on a bed of sand ripples on the seafloor (Heezen and Hollister, 1971), bubble plumes from occasional breaking waves under a rough sea surface (Woolf, 1997), isolated or small groups of trees in a field of bushes or grassland (Greig-Smith, 1984), etc.

The spatial distribution concerning two different types of scatterers in an aggregation is illustrated for two distinct cases, one in which the two types are separated into subregions [Fig. 3-1(a)] and the other in which the two types are interspersed [Fig. 31(b)]. In each case, the size of the resolution cell of the sonar/radar system is much smaller than the size of the analysis window and, in the first case, also much smaller than the size of each subregion. Thus, in the first case, in any one ping, only one type of scatterer is "seen" by the system [Fig. 3-1(a)]. As a result, the echo statistics for the pings included in the analysis window containing both subregions are nonstationary across the two subregions (Abraham et al., 2011). The second case involves the "mixed assemblage" which is the focus of this paper [Fig. 3-1(b)]. Here, the two types of scatterers are interspersed and uniformly distributed so that the echo statistics within the analysis window are stationary. Both scenarios are prevalent in nature and require different statistical models. Note that the term "monotype aggregation" will be used to refer the cases in which only one type of scatterer is included in the analysis window that are opposite to the two cases described above. The terminology discussed here will be used in this chapter and throughout this thesis.

Many models have been proposed to characterize non-Rayleigh-distributed echoes. Statistical analysis of data usually involves fitting the data to a large pool of models to determine the best representation (e.g., Abraham and Lyons, 2010; Gallaudet and de Moustier, 2003; La Cour, 2004; Watts and Wards, 2010). However, since most of these models do not provide explicit connections between the model parameters and the underlying scattering mechanisms, statistical descriptions using this datadriven approach are often only applicable to data collected using similar systems in specific geographical locations. In other words, these models are neither predictive nor interpretive with respect to the variation of sonar/radar system parameters or 
the scattering processes in the environment.

Several other models (Abraham and Lyons, 2002; Crowther, 1980; Jakeman and Pusey, 1976; Middleton, 1999) do provide a link between the model parameters and the physical scattering environment by imposing specific assumptions on the scatterers in a resolution cell. Among them, the $K$-distribution has been successfully applied to data collected under a variety of contexts (Jakeman and Pusey, 1976; Ward et al., 2006). However, the link between the $K$-distribution model parameters and the environment was established under the assumption that the scattering amplitude of scatterers or scattering patches after beampattern effects is exponentially-distributed (Abraham and Lyons, 2002). This assumption is not realistic for many important scenarios in nature, including most biological aggregations in the sea (Lalli and Parsons, 1997).

Recognizing the potential nonstationarity of the echo samples included in the analysis window [Fig. 3-1(a)], the multiple-component mixture model (referred to as the " $M$-component mixture model" throughout this paper) has been used extensively to fit experimental data (e.g., Abraham et al., 2010, 2011; Chotiros, 2010; Gallaudet and de Moustier, 2003; Stanton and Chu, 2010; Ward and Tough, 2002). This model describes the echo distribution as a linear combination of multiple probability distributions through

$$
p_{A}^{\Sigma}(a)=\sum_{m=1}^{M} w_{m} p_{A, m}(a),
$$

where $a$ is the echo amplitude (or, more precisely, magnitude of complex echo), $M$ is the number of component pdfs, and $p_{A, m}(a)$ and $w_{m}$ are the pdf and proportion factor of the $m$ th component pdf, respectively, with the constraint $\sum_{m=1}^{M} w_{m}=1$.

Specifically for aggregations composed of more than one type of scatterer, each being in their own subregion, $M$ denotes the number of subregions in the aggregation, and the proportional factor $w_{m}$ is determined by the relative proportion of data included in the analysis window from the $m$ th subregion. Each component pdf can then be written as

$$
p_{A, m}(a)=p_{A, m}\left(a ; N_{m}, r_{m}\right),
$$


(a)

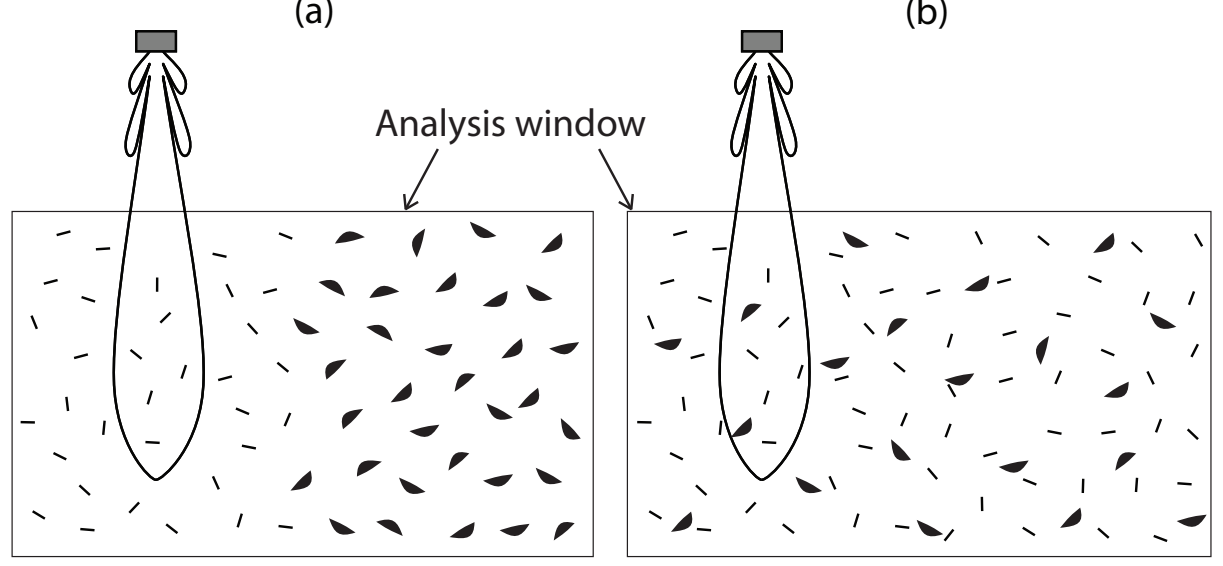

Figure 3-1: Illustration of analysis windows containing two possible spatial arrangements for aggregations composed of more than one type of scatterer. (a) Scatterers of the same type are separated into their own subregion. (b) Scatterers of different types are uniformly interspersed throughout the analysis window. In each case, the sonar/radar resolution cell is much smaller than the analysis window and, in case (a), is also much smaller than each subregion.

where $N_{m}$ and $r_{m}$ are the number of scatterers and relative scattering amplitude, respectively, of the $m$ th type of scatterer. Each $r_{m}$ is calculated relative to the amplitude of the weakest scatterer in the aggregation.

However, for mixed assemblages as depicted in Fig. 3-1(b), each echo sample is formed by a coherent sum of the echoes from each scatterer (regardless of type) contained in a single resolution cell (Chu and Stanton, 2010). Therefore, instead of a weighted sum over several component pdfs, the resultant echo pdf should be evaluated through a coherent summation of complex random variables, each associated with a scatterer, which can be written in the following parametrized form

$$
p_{A}^{\Sigma}(a)=p_{A}^{\Sigma}\left(a ; N_{1}, N_{2}, \ldots, N_{M}, r_{1}, r_{2}, \ldots, r_{M}\right) .
$$

In this study, the echo statistics associated with mixed assemblages [as represented in Fig. 3-1(b)] are formulated with this approach of coherently adding all echoes. This is a physics-based approach, beginning with the scattering physics of individual scatterers and incorporating effects due to their locations in the beam. Both the scattering by individuals and their locations in the beam are randomized. This is in contrast to 
the above-mentioned approaches in which parameters of the echo statistics are not directly linked to physical processes. The new formulation is a specialized version of the general formula for the echo pdf of an arbitrary number of scatterers given in Chu and Stanton (2010), which utilized the method of characteristic functions (CFs) proposed by Barakat (1974) to calculate the envelope pdf of the sum of random variables. The signals modeled are narrowband. The aforementioned beampattern effects are also incorporated explicitly in this formulation (Ehrenberg, 1972). Theoretical predictions made by this CF-based mixed assemblage pdf are validated by numerical simulations of echoes from mixed assemblages.

In addition, the performance of the CF-based mixed assemblage pdf as an inference tool is compared with that of the $M$-component mixture model in the case of mixed assemblages containing two types of scatterers [Fig. 3-1(b)] over a wide range of numerical densities and relative scattering amplitudes of the scatterers $\left[N_{m}\right.$ 's and $r_{m}$ 's in (3.1) and (3.3)]. Through a best-fit analysis, it is observed that only the CF-based mixed assemblage pdf is able to simultaneously fit the shape of the echo pdf of simulated data and accurately infer the parameters of the simulated mixed assemblage composition, while use of the $M$-component mixture model sometimes results in significant errors in parameter estimates under important conditions.

This chapter is organized as follows. In Sec. 3.2, the theoretical development of the CF-based mixed assemblage pdf and the incorporation of beampattern effects are presented. In Sec. 3.3, the procedures of generating theoretical curves and numerical simulations are discussed, and important examples of numerically-validated CFbased mixed assemblage pdfs are shown. The performances of the CF-based mixed assemblage pdf and the $M$-component mixture model are compared in Sec. 3.4. The summary and conclusion of this study are given in Sec. 3.5. 


\subsection{Theoretical development of characteristic func- tion (CF)-based mixed assemblage pdf}

In this section, a general expression is derived to describe the echo envelope pdf for mixed assemblages as a function of the numerical density and scattering amplitude of each type of scatterer, as well as the number of scatterer types. This expression is general and applicable to the case in which the scatterers in the analysis window are of arbitrary type. The derivation follows the method of characteristic functions (Barakat, 1974) to obtain the envelope statistics for the sum of generic complex random variables. The beampattern effects, which are of particular concern for radar/sonar applications, are also incorporated explicitly in the formulation (Chu and Stanton, 2010; Ehrenberg, 1972). Some derivations from previous work are summarized here as they will be used in the final formulation.

\subsubsection{Problem setup}

In a mixed assemblage, different types of scatterers are uniformly interspersed among one another, as depicted in Fig. 3-1(b). The geometry involves direct paths between the radar/sonar and the scatterers in the backscattering direction, with no reflections from the boundaries such as the seafloor and sea surface.

Assuming the use of narrowband signals, the echo voltage $V_{i}$ received through the sonar/radar system associated with each scatterer can be modeled as a narrowband continuous wave (CW) signal and described in (complex) phasor form,

$$
V_{i}=a_{i} e^{j\left(\theta_{i}+\omega_{a} t\right)}
$$

where $\omega_{a}$ is the angular frequency, $j=\sqrt{-1}$, and $a_{i}$ and $\theta_{i}$ are the echo magnitude and relative phase associated with the $i$ th scatterer, respectively. All $a_{i}$ and $\theta_{i}$ are statistically independent. The magnitude of each echo, $a_{i}$, depends upon the size, shape, orientation, and material properties of the $i$ th scatterer, as well as the frequency and the location of the $i$ th scatterer in the beam. The dependence of $a_{i}$ upon the loca- 
tion of the $i$ th scatterer in the beam is implicit here, and will be given in detail in Sec. 3.2.3. Since the path differences induced by the position of the scatterers within a resolution cell are assumed to be greater than the wavelength of the incidence wave, and the locations of the scatterers are assumed to be random and independent of one another, $\theta_{i}$ can be modeled as a random variable uniformly distributed over the range of $[0,2 \pi)$.

For the case of $N$ scatterers randomly located in the half-space insonified/irradiated by the sonar/radar beam, the total echo (voltage) measured through the system, $V^{\Sigma}$, can be expressed as a sum of the contributions from individual scatterers, i.e.,

$$
V^{\Sigma}=\sum_{i=1}^{N} V_{i}=e^{j \omega_{a} t} \sum_{i=1}^{N} a_{i} e^{j \theta_{i}}
$$

The echoes from all scatterers in the half-space overlap due to the infinite duration of the assumed CW signals. Note, however, that the actual contribution of each scatterer to the total echo is determined by the location of the scatterers in the beampattern of the transducer. The scatterers located in the nulls of the beampattern do not contribute to the total echo, and the contributions from scatterers located in the sidelobes are much lower than the contributions from scatterers located in the mainlobe. Therefore, the number of scatterers located within a resolution cell (see definition in Sec. 3.1) is generally much less than $N$. The echo pdf analyzed in this paper deals with fluctuations of envelope amplitude (i.e., magnitude of complex signal) of the total field, $a=\left|V^{\Sigma}\right|$, and its distribution will be denoted by $p_{A}^{\Sigma}(a)$ throughout this chapter.

\subsubsection{Method of characteristic functions - beampattern ef- fects not explicit}

The above formulation for the total echo measured through a sonar system is the same as the general case described by Barakat (1974) in which a finite number of independent sinusoidal waves with arbitrary magnitudes and random phases are summed 
coherently. He derived a useful formula to express the pdf of the resultant echo magnitude as a Fourier-Bessel series expansion of the product of characteristic functions (CF, Papoulis, 2002) associated with the pdf of the real or imaginary part of individual phasors. The method is summarized below.

Define $y_{i}$ to be the real part of the scattering contribution from the $i$ th scatterer and $p_{A, i}(a)$ to be the distribution of its magnitude. Since $y_{i}=\operatorname{Re}\left\{V_{i}\right\}=a_{i} \cos \theta_{i}$, $y_{i}$ can be viewed as the product of two independent random variables, $a_{i}$ and $\cos \theta_{i}$. Through manipulation of the product of two random variables, the distribution of $y_{i}$ is shown to be [eq.(13) in Barakat (1974)]

$$
p_{y_{i}}\left(y_{i}\right)= \begin{cases}\frac{1}{\pi} \int_{\left|y_{i}\right|}^{\infty} \frac{p_{A, i}\left(a_{i}\right)}{\left(a_{i}^{2}-y_{i}^{2}\right)^{\frac{1}{2}}} d a_{i} & ,\left|y_{i}\right| \leq a_{i} \\ 0 & ,\left|y_{i}\right|>a_{i}\end{cases}
$$

Define $y^{\Sigma}$ to be the real part of the total echo field, $y^{\Sigma}=\operatorname{Re}\left\{V^{\Sigma}\right\}$, its pdf can be expressed as the convolution of pdfs of the real part of individual echoes,

$$
p_{y^{\Sigma}}=p_{y_{1}} * p_{y_{2}} * p_{y_{3}} * \cdots
$$

Define $\phi_{i}$ to be the CF of $p_{y_{i}}\left(y_{i}\right)$ [eq.(21) in Barakat (1974)],

$$
\phi_{i}(\omega)=\operatorname{FT}\left\{p_{y_{i}}\left(y_{i}\right)\right\}=\int_{-\infty}^{\infty} p_{A, i}\left(a_{i}\right) J_{0}\left(\omega a_{i}\right) d a_{i}
$$

where $\mathrm{FT}\{\cdot\}$ denotes the Fourier transform and $J_{\nu}(\cdot)$ denotes the cylindrical Bessel function of the first kind of order $\nu$. Since the convolution of pdfs corresponds to the product of CFs, the CF of the pdf of the real part of the total echo field from $N$ scatterers, $\phi^{\Sigma}$, is

$$
\phi^{\Sigma}(\omega)=\prod_{i=1}^{N} \phi_{i}(\omega)
$$

By considering $y^{\Sigma}$ as a projection of the vector $\left(\operatorname{Re}\left\{V^{\Sigma}\right\}, \operatorname{Im}\left\{V^{\Sigma}\right\}\right)$ onto the real axis in the complex plane and using the fact that $\operatorname{Re}\left\{V^{\Sigma}\right\}$ and $\operatorname{Im}\left\{V^{\Sigma}\right\}$ have the same amplitude distribution, the pdf of the length of this vector, $a$, can be written in the 
form of a Fourier-Bessel series [eq.(55-56) in Barakat (1974)]

$$
p_{A}^{\Sigma}(a)=2 a \sum_{n=1}^{\infty} \frac{\phi^{\Sigma}\left(\frac{\eta_{n}}{a_{\max }}\right)}{\left[a_{\max } J_{1}\left(\eta_{n}\right)\right]^{2}} J_{0}\left(\eta_{n} \frac{a}{a_{\max }}\right),
$$

where $a_{\max }$ is the maximum echo amplitude, and $\eta_{n}(n=1,2, \ldots)$ are the positive roots of $J_{0}(x)=0$. The combination of (3.9) and (3.10) can be used to calculate the echo pdf of an aggregation of an arbitrary number of scatterers with arbitrary scattering amplitudes. Note that since $\phi^{\Sigma}$ decreases rapidly and oscillates while approaching zero asymptotically, in numerical implementations of (3.10), the sum of the infinite series can be truncated at the location where the first zero in $\phi^{\Sigma}$ is encountered. The number of terms included is determined by the range of $a$ where the values of $p_{A}^{\Sigma}(a)$ are not negligible. This is analogous to the time-frequency reciprocity in Fourier analysis, where the support of the temporal and spectral representations of a given signal are approximately the inverse of each other.

\subsubsection{Incorporating beampattern effects}

Since each $a_{i}$ is measured through the radar/sonar system, the location of the $i$ th scatterer in the beam will determine the weighting factor applied by the radar/sonar system to the scattering amplitude of the echo. These beampattern effects can be explicitly accounted for when forming $p_{A, i}\left(a_{i}\right)$. For a single scatterer randomly located in the beam, the echoes "seen" through the receiver of the system (i.e., including beampattern effects) can be viewed as a product of two random variables, $s_{i}$ and $b$, where $s_{i}$ is the magnitude of the scattering amplitude of the $i$ th scatterer, and $b$ is the random weighting factor imposed by the beam. The echo envelope pdf of the $i$ th scatterer as measured through the receiver can be expressed as

$$
p_{A_{i}}\left(a_{i}\right)=\int_{a_{i}}^{\infty} \frac{1}{s_{i}} p_{S_{i}}\left(s_{i}\right) p_{B}\left(\frac{a_{i}}{s_{i}}\right) d s_{i},
$$

where $p_{S_{i}}\left(s_{i}\right)$ is the distribution of the magnitude of the scattering amplitude of the $i$ th scatterer (i.e., the echo amplitude before the beampattern effects), and $p_{B}(b)$ 
is the beampattern pdf [originally derived in (Ehrenberg, 1972), with subsequent papers summarized in (Chu and Stanton, 2010)]. For a system with an axisymmetric beampattern,

$$
p_{B}(b)=\sum_{l} \frac{p_{\Psi}\left(\psi_{l}(b)\right)}{\left|\frac{d b}{d \psi}\right|_{\psi_{l}(b)}},
$$

where $\psi$ is a random variable representing the polar angle between the scatterer position and the axis of the beam, and each $\psi_{l}$ is a root to $b=b(\psi)$. All simulations and analyses carried out in this chapter are conducted under the assumption of an axisymmetric transceiver aperture with $k a_{T}=2 \pi$, where $k$ is the wavenumber and $a_{T}$ is the aperture radius. This corresponds to a beamwidth of approximately $30^{\circ}$. For a more complicated beampattern with both polar and azimuthal angular dependence, such as a transducer with rectangular aperture, (3.12) has to be modified, but the same general formulation in (3.11) is applicable.

\subsubsection{Echoes from mixed assemblages}

The above CF-based approach can be used to calculate the echo pdf associated with mixed assemblages through a simple modification of (3.9). In the mixed assemblage, assume that there are $M$ types of scatterers, each with $N_{m}(m=1,2, \ldots, M)$ scatterers randomly and uniformly distributed in the half space. Using (3.9), the CF of the pdf of the real part of the assemblage echo can be expressed as

$$
\phi^{\Sigma}(\omega)=\prod_{m=1}^{M}\left(\phi_{m}(\omega)\right)^{N_{m}}
$$

where $\phi_{m}$ denotes the CF of a single scatterer of type $m$ and can be obtained by (3.8). The total echo pdf can then be calculated using (3.10).

The CF-based mixed assemblage pdf calculated using (3.10) and (3.13) rigorously incorporates important parameters associated with the system and scatterers: 1) beampattern effects associated with the random locations of the scatterers in the beam; 2) statistics of the random scattering amplitude (before beampattern effects) of each scatterer; and 3) number of each type of scatterer. Each of these parameters 
are all accounted for explicitly.

\subsection{Numerical validation and examples of CF-based mixed assemblage pdfs}

In this section, the theoretical CF-based mixed assemblage pdf derived in Sec. 3.2 is validated by simulated data. The effects of the change of mixed assemblage composition on the shape of the echo pdf are also examined.

As seen in (3.13), two of the parameters controlling the CF-based mixed assemblage pdf are the number of scatterer types, $M$, and the number of each type of scatterers, $N_{m}$. A third model parameter is the relative, rather than absolute, scattering amplitudes of the different types of scatterers. This is because the shapes of echo pdfs are dictated by the composition of mixed assemblages, and the absolute echo amplitudes only change the location of the pdfs on the amplitude axis. Therefore, the pdfs will be plotted vs the echo amplitude normalized by the root-mean-square (rms) amplitude following the procedure detailed in Appendix A. This normalization procedure is conceptually analogous to normalizing normal distribution curves with the same shape (and thus the same standard deviation) but different mean values to the standard normal distribution.

As discussed in Sec. 3.2, the magnitude of the scattering amplitude of each scatterer is specified by a distribution (e.g., $p_{A, i}(a)$ for the $i$ th scatterer), instead of a fixed value. Furthermore, it is the relative scattering amplitudes, rather than their absolute levels, among the different types of scatterers that are important to the shape of the echo pdf (associated with either individual or aggregation echoes). Therefore, the statistics of the scattering amplitude of the $m$ th type of scatterer can be described through use of the ratio

$$
r_{m}=\frac{\lambda_{m}}{\lambda_{1}}
$$

where $\lambda_{m}$ and $\lambda_{1}$ are the rms magnitudes of the scattering amplitudes of the $m$ th type of scatterer and the weakest scatterer in the assemblage, respectively $(m=$ 
$\left.1,2, \ldots, M ; \lambda_{M}>\lambda_{M-1}>\ldots>\lambda_{1}\right)$. In this study, all simulated data and theoretical curves are generated under the assumption that the magnitude of scattering amplitude of each individual scatterer (i.e., the echo before beampattern effects) is Rayleighdistributed. In this case, $\lambda_{m}$ is proportional to the mean of the distribution of the magnitude of the scattering amplitude of the $m$ th type of scatterer. Because the quantity $\lambda_{m}^{2}$ is a measure of energy, $r_{m}^{2}$ is equal to the ratio of the mean of the backscattering cross section (square of magnitude of scattering amplitude) of the $m$ th type of scatterer to that of the weakest scatterer in the assemblage.

To illustrate the effects of mixed assemblage composition on the shape of the echo pdf, particularly on the non-Rayleigh elevated tail of the pdf, theoretical curves are generated for mixed assemblages composed of only two types of scatterers $(M=$ 2). For simplicity, these two types of scatterers will be referred to as "strong" and "weak" scatterers, with the number of each type of scatterer denoted by $N_{s}$ and $N_{w}$, respectively. The ratio of the magnitudes of the scattering amplitudes of the strong to the weak scatterers, $r_{2}$, is specially denoted by $r_{s w}$ in this case. The phase of each individual scatterers is assumed to be uniformly distributed over the range of $[0,2 \pi)$ for all simulated data and theoretical curves.

\subsubsection{Simulated data generation}

Following (3.14), the echoes from the $m$ th type of scatterer (before the beampattern effects) are simulated by drawing samples from Rayleigh distributions with parameter $r_{m}$, using the convention below

$$
f_{R}\left(x ; r_{m}\right)=\frac{x}{r_{m}^{2}} e^{-x^{2} / 2 r_{m}^{2}}
$$

The magnitudes of these echoes are further modulated according to the random locations of the scatterers in the beam. In the present case when a circular aperture is considered, the random location refers to the polar angle of the scatterer to the axis of the beam. The phase of the echo from each individual scatterer is generated by drawing samples from a uniform distribution over the range of $[0,2 \pi)$. 
Each independent realization of the ensemble is formed by coherently summing $\sum_{m=1}^{M} N_{m}$ random phasors with the above magnitudes and phases. The echo pdf of the simulated data is obtained through forming the number frequency histogram of simulated realizations that have been normalized following the procedure described in Appendix A. In general, $10^{3}$ realizations are sufficient to form the echo pdf with reasonable accuracy over a wide range of echo amplitudes. However, with the focus to accurately predict the tail of the pdfs, all simulated data presented in this paper contain $10^{5}$ realizations.

\subsubsection{CF-based mixed assemblage pdf generation}

The echo pdf of a single scatterer of type $m, p_{A, m}\left(a ; N_{m}=1, r_{m}\right)$, is required to calculate the CF for an arbitrary number of scatterers using (3.8) and (3.9). Since the shape of the echo pdf is only affected by the assemblage composition regardless of the absolute scattering level, $p_{A, m}\left(a ; N_{m}=1, r_{m}\right)$ can be obtained by scaling $p_{A, 1}\left(a ; N_{1}=1, r_{1}\right)$ on the echo amplitude axis so that the ratio of the rms amplitude of $p_{A, m}\left(a ; N_{m}=1, r_{m}\right)$ to that of $p_{A, 1}\left(a ; N_{1}=1, r_{1}\right)$ equals to $r_{m}$. Theoretical echo pdfs of mixed assemblages are then generated by first obtaining the total CFs through the combination of (3.8) and (3.13), and converting the total CFs to the total echo pdf using (3.10).

\subsubsection{Validation of CF-based mixed assemblage pdf (as a pre- dictor)}

Theoretical predictions given by the CF-based mixed assemblage pdf are validated by numerical simulations generated over a wide range of ratios of scattering amplitude and numbers of scatterer types (Fig. 3-2). It is observed that the accuracy of the predicted echo pdfs is related to the accuracy of the echo pdf of a single weakest scatterer, $p_{A, 1}\left(a ; N_{1}=1, r_{1}\right)$, due to the procedure described in Sec. 3.3.2. Specifically, inaccuracy in the region in which the values of echo amplitude are low and probability densities are high reduces the accuracy of the resultant mixed assemblage pdf, because 


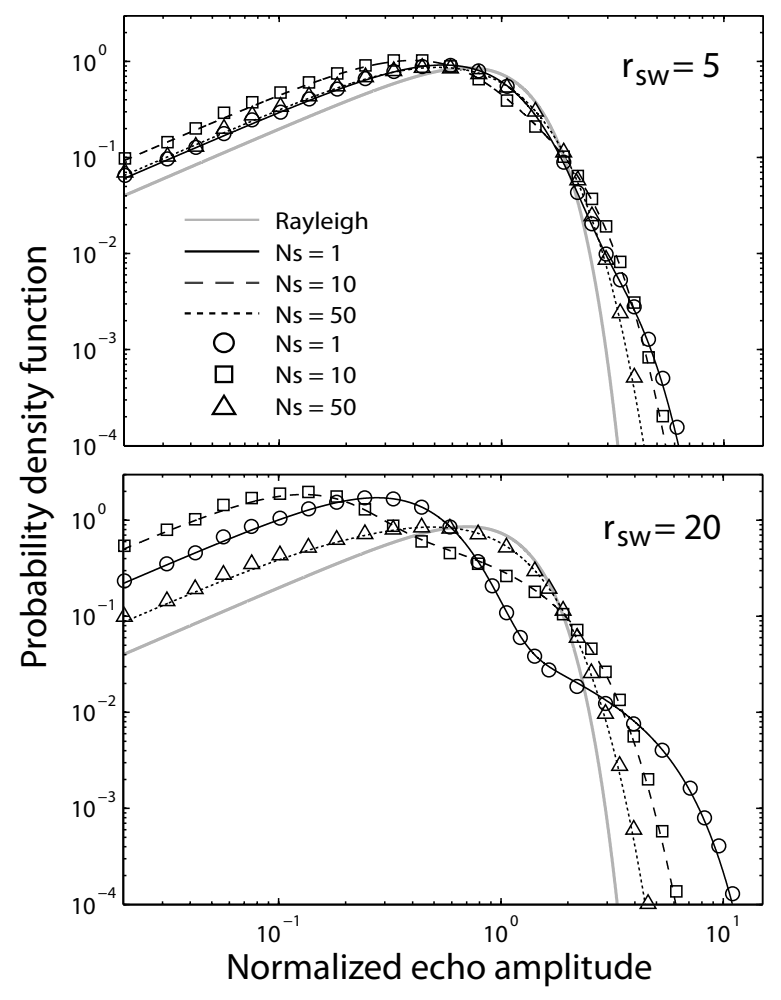

Figure 3-2: Validation of the theoretical CF-based mixed assemblage pdf using (3.10) and (3.13) (lines) with numerically-simulated echo pdfs (symbols) for the case of two types of scatterers uniformly interspersed as in Fig. 3-1(b). The number of weak scatterers, $N_{w}$, is fixed at 100 whereas the number of strong scatterers, $N_{s}$, and the ratio of the magnitudes of scattering amplitudes of the strong to the weak scatterers, $r_{s w}$, are both varied.

energy contained in this region can affect the rms value used for normalization. This is due to numerical issues with small numbers.

The difference between the echo pdfs of monotype aggregations and mixed assemblages depends on the composition of the aggregation (Fig. 3-3). For example, when the $N_{s}$ and $r_{s w}$ are of moderate value (e.g., $N_{s}=5,10$ and $r_{s w}=5$ ), both the tail and the overall shape of the CF-based mixed assemblage pdfs differ from those of monotype aggregations. However, as $N_{s}$ and $r_{s w}$ increase, the total scattered field is increasingly dominated by the strong scatterers, results in the almost identical shape of the tails (e.g., CF-based mixed assemblage pdfs with $r_{s w}=20$ ). 


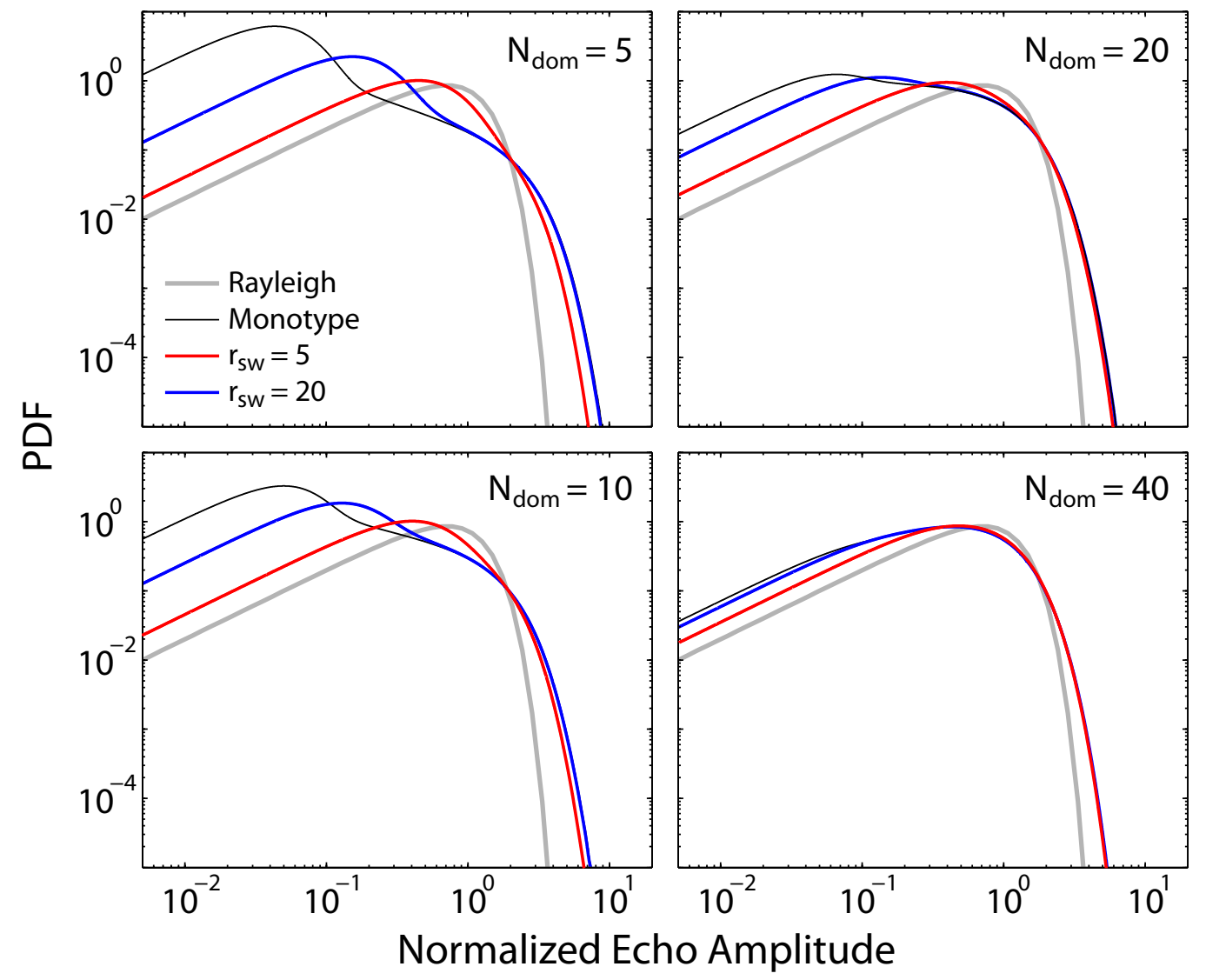

Figure 3-3: Comparison of echo pdfs from monotype aggregation and mixed assemblages with varying number of scatterers and $r_{s w}$. The number of dominant scatterers, $N_{\text {dom }}$, is indicated for each plot. $N_{d o m}$ equals the total number of scatterers in monotype aggregations the number of strong scatterers in mixed assemblages (i.e., $\left.N_{\text {dom }}=N_{s}\right) . N_{w}$ is fixed at 100 for all mixed assemblages. 


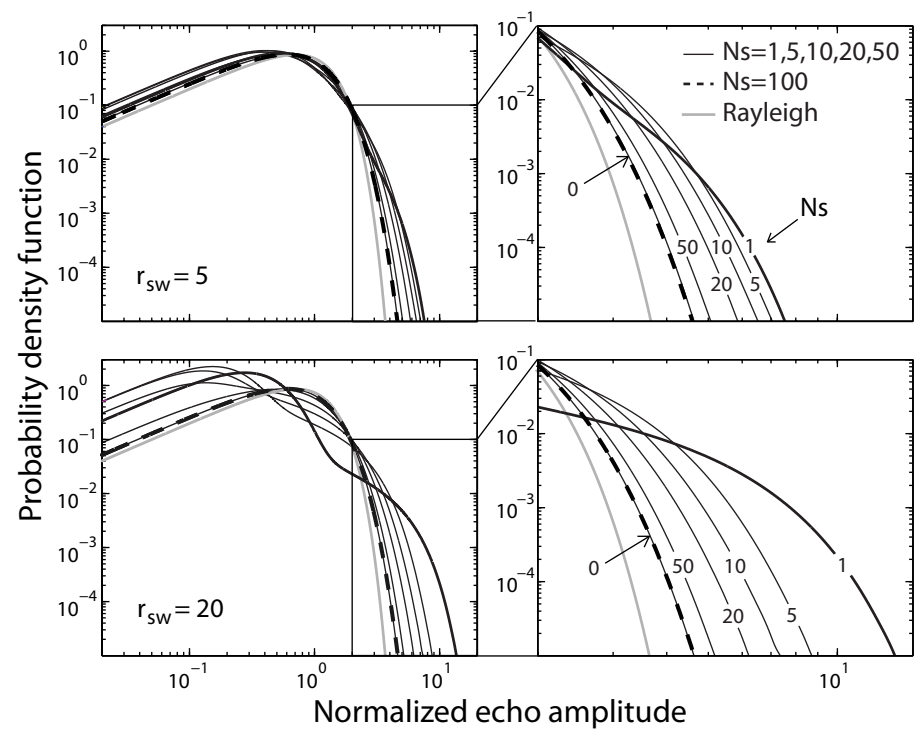

Figure 3-4: Variation of the CF-based mixed assemblage pdf as a function of mixed assemblage composition. The top plots show the effect of changing $N_{s}$ $(=1,5,10,20,50,100)$ on the shape of the echo pdf when $r_{s w}=5$ and $N_{w}=100$. The bottom plots show the echo pdf variation with the same combinations of $N_{s}$ and $N_{w}$, but with $r_{s w}=20$.

\subsubsection{Effect of mixed assemblage composition on the echo pdf}

The effects of mixed assemblage composition on the shape of the echo pdfs are examined in cases consisting of only two types of scatterers.

\section{Varying the ratio of scattering amplitudes}

The shape of the echo pdf is shown to change by varying $r_{s w}$ while keeping $N_{s}$ and $N_{w}$ fixed (Fig. 3-4). When $r_{s w}$ is small (top panel), the echo contributions from the strong scatterers are more confined to the tail of the pdfs. The lower amplitude portion of the pdfs, which is dominated by the weak scatterers, remains relatively stable. However, when $r_{s w}$ is increased (bottom panel), the scattered field is increasingly dominated by the strong scatterers, and the energy contained in the tail of the pdfs is of a higher proportion of the total scattered energy. Therefore, in addition to an elevated heavy tail, the modes of the normalized pdfs with larger $r_{s w}$ are displaced toward smaller echo amplitudes. 


\section{Varying the number of scatterers in each type}

The shape of the echo pdf also varies with changing $N_{w}$ and $N_{s}$ while holding $r_{s w}$ fixed (Fig. 3-4). When $N_{s}$ is small, the curves are highly non-Rayleigh owing to occasional large echoes from the strong scatterers occuring in the mainlobe of the beam. As $N_{s}$ increases, the shape of the echo pdf gradually approaches the Rayleigh distribution. This trend of variation from non-Rayleigh toward the Rayleigh distribution with increasing number of scatterers is the same as the results reported in Chu and Stanton (2010) for assemblages consisting of only one type of scatterer. Furthermore, as $N_{s}$ increases, the total scattered field is increasingly dominated by the strong scatterers, and the contribution from the weak scatterers becomes negligible. This explains why echo pdfs with the combination $\left(N_{s}=0, N_{w}=100\right)$ and $\left(N_{s}=100, N_{w}=100\right)$ are almost identical, regardless of the value of $r_{s w}$. This property has an impact on the use of echo pdfs to infer the composition of mixed assemblages, as will be discussed in Sec. 3.4.3.

\subsection{Comparison of the CF-based mixed assemblage pdf and $M$-component mixture model}

In this section, the performance of the newly developed CF-based mixed assemblage pdf is compared with the commonly used $M$-component mixture model in their use to infer parameters of mixed assemblages. The echo pdfs of simulated data are fit to model pdfs with varying parameters through a curve-fitting procedure. The parameters of the assemblage composition corresponding to the best-fitting pdfs (i.e., the inferred parameters) are then compared with the true parameters used in numerical simulations. The discrepancies between the true and inferred parameters for each model are compared. To simplify the analysis, the number of scatterer types is limited to two $(M=2)$ for all calculations as in the earlier examples.

The simulated data are generated following the procedure described in Sec. 3.3.1. The number of weak scatterers, $N_{w}$, is fixed at 100 and the number of strong scatter- 
ers, $N_{s}$, is varied between 1 and 100. The ratio of magnitude of scattering amplitude of the strong to the weak scatterers, $r_{s w}$, is varied from 5 to 60 for all combinations of $N_{w}$ and $N_{s}$. In order to test the sensitivity of the curve-fitting procedure to small variations in the echo pdfs of simulated data, ten sets of simulated data, each with $10^{5}$ independent realizations, are generated for each combination of parameters. Since the results from all simulated data sets are similar, only one representative example is shown in this chapter.

\subsubsection{Echo statistic models formulated for two types of scat- terers}

Using the general formulations (3.1) and (3.3) in Sec. 3.1, the CF-based mixed assemblage pdf and the $M$-component mixture model are given here in simplified forms for the case of only two types of scatterers. From (3.3), the CF-based mixed assemblage pdf for two types of scatterers is

$$
p_{A}^{\Sigma}(a), \text { CF-based }=p_{A}^{\Sigma}\left(a ; N_{w}, N_{s}, r_{s w}\right)
$$

From (3.1), the 2-component mixture model is

$$
p_{A}^{\Sigma}(a), \text { mixture }=\left(1-w_{s}\right) p_{A, w}(a)+w_{s} p_{A, s}(a)
$$

where $p_{A, w}(a)$ and $p_{A, s}(a)$ are the component pdf for the weak and strong scatterers, respectively, and $w_{s}$ is the proportion of strong scatterers in the echo samples.

As with the more general (3.1) and (3.3), (3.16) is from a coherent summation of the contribution from both the strong and weak scatterers, whereas (3.17) considers the total echo distribution as a sum of two independent scattering processes, with each sample drawn from either of the two processes.

Two versions of the 2-component mixture model are used in this model comparison study. In the first version, the component pdfs are Rayleigh distributions with different powers of a ratio of $r_{s w}$. The use of a Rayleigh distribution implies that 
each echo sample is from a large number of unresolvable scatterers filling the beam, i.e., $N_{w} \rightarrow \infty$ and $N_{s} \rightarrow \infty$. This version of the 2-component mixture model will be referred to as the "2-component Rayleigh mixture model".

In the second version, the component pdfs are calculated using the CF-based echo pdf formulation, but with only one type of scatterer in each component. Specifically, $p_{A, w}(a)$ and $p_{A, s}(a)$ are calculated using (3.10) and

$$
\phi_{w}^{\Sigma}=\left(\phi_{w}(\omega)\right)^{N_{w}} \quad \text { and } \quad \phi_{s}^{\Sigma}=\left(\phi_{s}(\omega)\right)^{N_{s}}
$$

This version of the 2-component mixture model will be referred to as the "2-component CF-based mixture model". Note that these two mixture models contain an additional parameter, the proportional factor, $w_{s}$, which does not have a physical meaning in the context of mixed assemblages, where the different types of scatterers are uniformly interspersed [Fig. 3-1(b)].

\subsubsection{Method for inferring parameters of mixed assemblage}

The composition of each simulated mixed assemblage is inferred by fitting the simulated data to large pools of echo pdfs generated by the above models. The parameters of the best-fitting model pdf are taken as the inferred parameters of the mixed assemblage composition. The best-fitting pdf is chosen by minimizing the Kullback-Leibler (KL) divergence (Kullback, 1959) between the echo pdf of the simulated data and model (theoretical) echo pdfs. The KL divergence calculates the overall mismatch between the echo pdfs from the model and data, while emphasizing the mismatch between the tails of the two pdfs through its use of a logarithm in the formulation (Stanton and Chu, 2010):

$$
\mathrm{D}_{\mathrm{KL}}=\int p_{\text {model }}(x) \log \frac{p_{\text {model }}(x)}{p_{\text {data }}(x)} d x
$$

where $p_{\text {model }}(x)$ and $p_{\text {data }}(x)$ are the echo pdfs of the model and simulated data, respectively. The emphasis on the tail is often preferred for echo pdf analysis, because 
statistical description of the tail is usually of particular interest in the applications, whereas the region with low echo amplitude is often contaminated by noise.

\subsubsection{Performance of models as inference tools}

The performances of the CF-based mixed assemblage pdf and the two 2-component mixture models in their use to infer parameters of mixed assemblages are evaluated in this section. The evaluation is based on the fit of the shape of the echo pdfs (Fig. 3-5) and the accuracy of inferred parameters of assemblage composition (Fig. 3-6).

Several examples of the echo pdf of simulated mixed assemblages and corresponding best-fitting model pdfs are illustrated (Fig. 3-5). The shape of the best-fitting CF-based mixed assemblage pdf fits successfully to the simulated data in all investigated cases. In contrast, the 2-component Rayleigh mixture model generally failed to produce the appropriate shape fit to the simulated data in all cases, most notably on the level near the "inflection points" and the slope of the tails (indicated by arrows in Fig. 3-5). This mismatch in shape is a direct result of the inadequacy of using the Rayleigh distribution to describe the scattering from a finite number of scatterers with associated beampattern effects. When CF-based pdfs are used as component pdfs in the mixture model (i.e., the 2-component CF-based mixture model), the shape of model echo pdfs fits the simulated data in most cases, despite small deviations near the inflection point or in the tail in some cases.

The results of inferred parameters of the assemblage composition for one representative set of simulated data with all investigated combinations of $N_{w}, N_{s}$, and $r_{s w}$ are illustrated (Fig. 3-6). For both the CF-based mixed assemblage pdf and the 2-component CF-based mixture model, the pools of model pdfs contain some cases in which there is only one CF-based pdf component, i.e., $N_{w}=0$ in (3.16) and (3.18), and $w_{w}=0, w_{s}=1$ in (3.17). When the data are best-fitted by the one-component CF-based model, the symbol is changed from a triangle $(\triangle)$ to a square $(\square)$ in the CF-based mixed assemblage pdf case, and from a circle $(\bigcirc)$ to a cross $(\times)$ in the 2-component CF-based mixture model case. In these cases, $r_{s w}$ is set to 0 .

Only the CF-based mixed assemblage pdf is able to accurately infer the composi- 

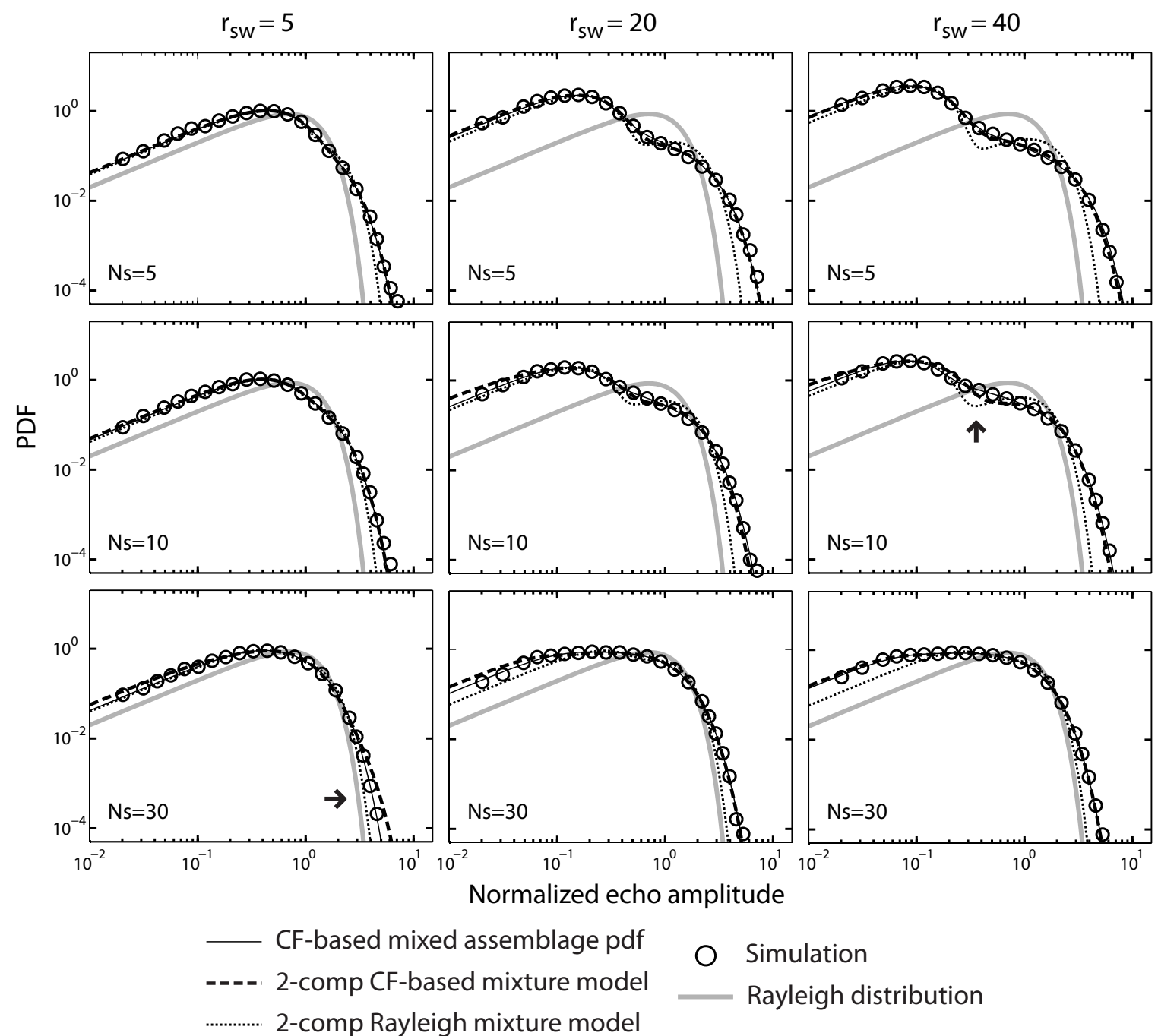

Figure 3-5: Several representative examples of the echo pdf of simulated mixed assemblages $(\bigcirc)$ and the corresponding best-fitting model pdfs (lines). Also shown on the plots are the true values of $r_{s w}$ and $N_{s}$ used to generate the simulated data. The best-fitting assemblage composition parameters for the models are summarized in Fig. 3-6. The arrows indicate the locations where the best-fitting mixture models have noticeable divergence from the data. 

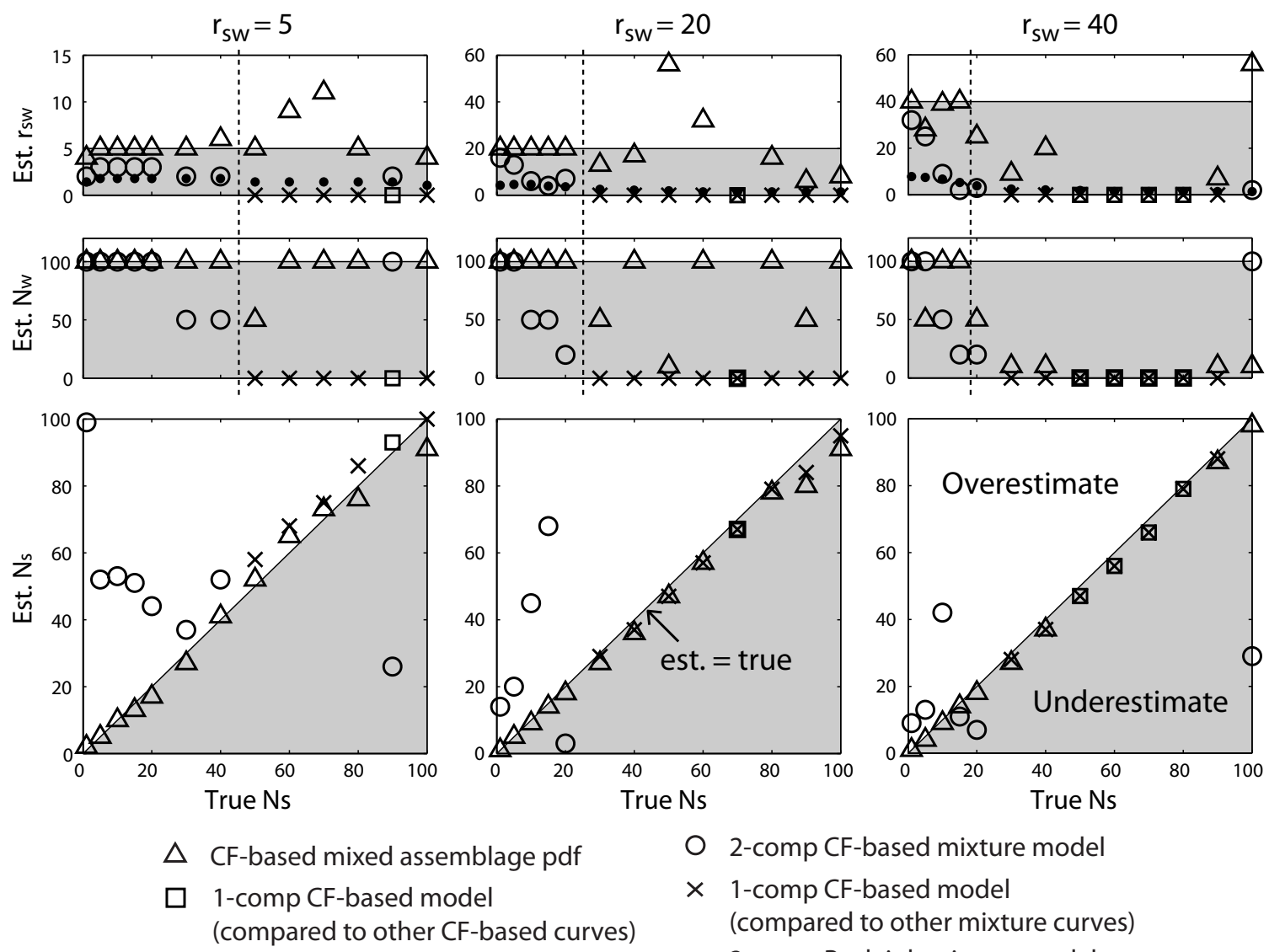

2-comp CF-based mixture model

$\times \quad$ 1-comp CF-based model (compared to other mixture curves)

- 2-comp Rayleigh mixture model

Figure 3-6: Comparison of the best-fitting assemblage composition parameters versus the true parameters for the CF-based mixed assemblage pdf $(\triangle)$, the 2-component CF-based mixture model $(\bigcirc)$, and the 2-component Rayleigh mixture model $(\bullet)$. The squares $(\square)$ and crosses $(\times)$ indicate the cases when the best-fitting models are composed of only one CF-based pdf component. The vertical dashed lines show the approximate locations where the performance of the CF-based mixed assemblage pdf as an inference tool starts to degrade. Note that the 2-component Rayleigh mixture model does not contain parameters $N_{w}$ and $N_{s}$. 
tion of simulated mixed assemblages (Fig. 3-6). Furthermore, the accuracy of this pdf is limited to a subset of conditions explored. The inferred parameters are the most accurate when the true $N_{s}$ is small. The model performance degrades as $N_{s}$ increases and as the shape of the echo pdf correspondingly approaches the Rayleigh distribution. Similar degradation in model performance is also observed for cases with large $r_{s w}$ (by comparing the locations of the vertical dashed lines for cases with different $r_{s w}$ in Fig. 3-6). In extreme cases where both $N_{s}$ and $r_{s w}$ are high, the best-fitting curves are often one-component CF-based models.

These trends of accuracy degradation can be explained by the fact that when $N_{s}$ or $r_{s w}$ is large, the echo samples are dominated by the strong scatterers, and the contribution from the weak scatterers becomes negligible. In these cases, the CFbased mixed assemblage pdfs with the same $N_{s}$ are very similar in the tails with only incremental differences in the low echo amplitude region (Fig. 3-7 and Fig. 3-8). In more general terms, the pdfs change the most when the number of dominant scatterers is small, thus, errors in inference increase as the incremental changes decrease. Note that these properties only apply in the analysis of normalized echo pdfs. Without normalization, echo pdfs in the above ambiguous cases will have similar shapes but different locations along the amplitude axis depending on the absolute scattering levels.

In contrast to the CF-based mixed assemblage pdf, up to order-of-magnitude errors result fromn the inference of mixed assemblage parameters when the other approaches involving mixture models are used. In addition to failing to produce an adequate fit for the shape of the echo pdf, the 2-component Rayleigh mixture model consistently underestimates $r_{s w}$ in all cases investigated (Fig. 3-6, top row). The 2-component CFbased mixture model, although producing a better fit on the shape of the echo pdf, gives erroneous estimation on all three parameters $N_{w}, N_{s}$ and $r_{s w}$. Specifically, when the true $N_{s}$ is small, this model overestimates $N_{s}$ and underestimates $r_{s w}$. Similar to the CF-based mixed assemblage pdf, the performance of the 2-component CF-based mixture model degrades significantly with increasing $N_{s}$ and $r_{s w}$.

In addition to producing erroneous inference results, the lack of physical meaning 


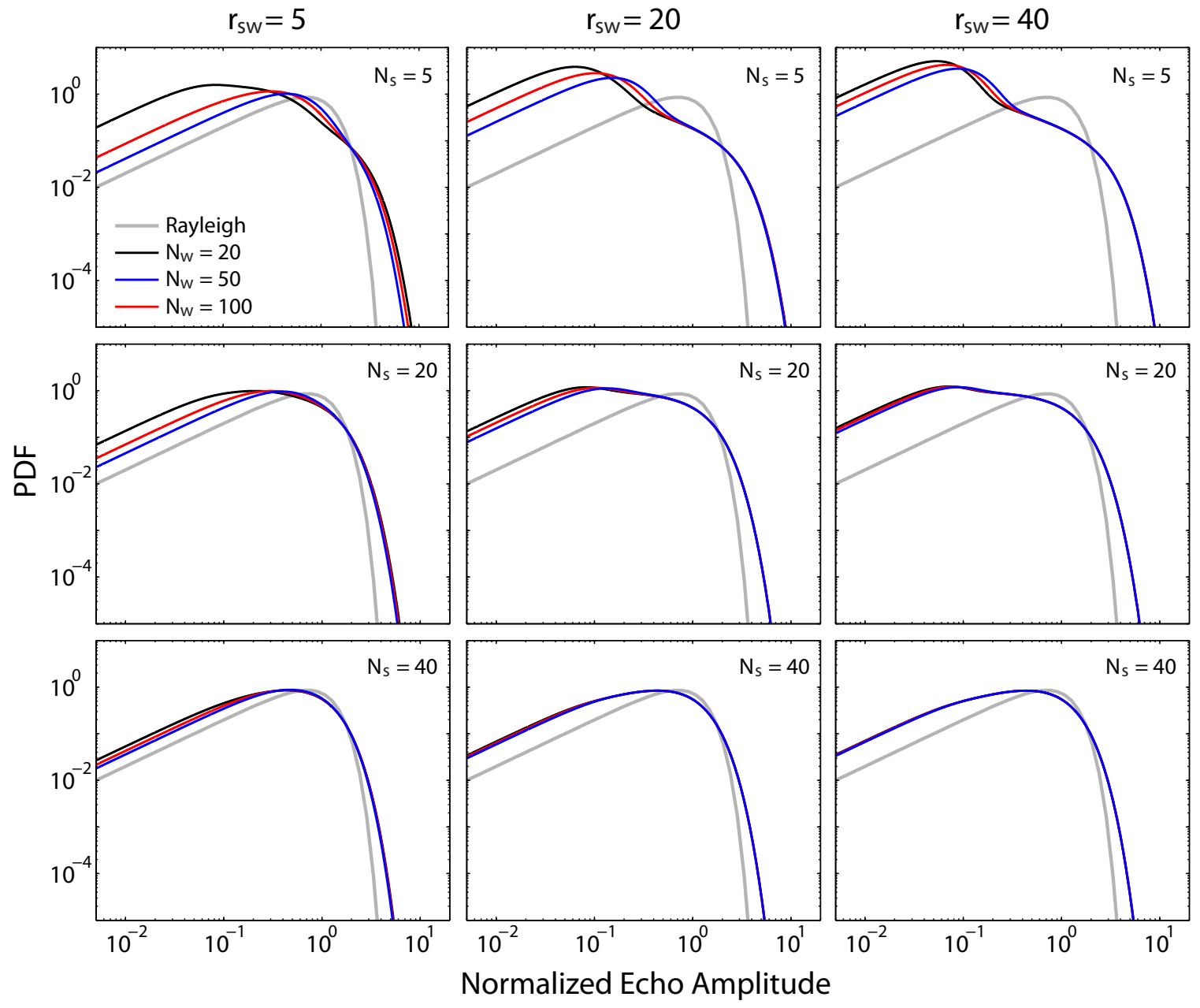

Figure 3-7: Comparison of CF-based mixed assemblage pdf produced with varying $N_{w}$ and fixed $N_{s}$ and $r_{s w}$. 


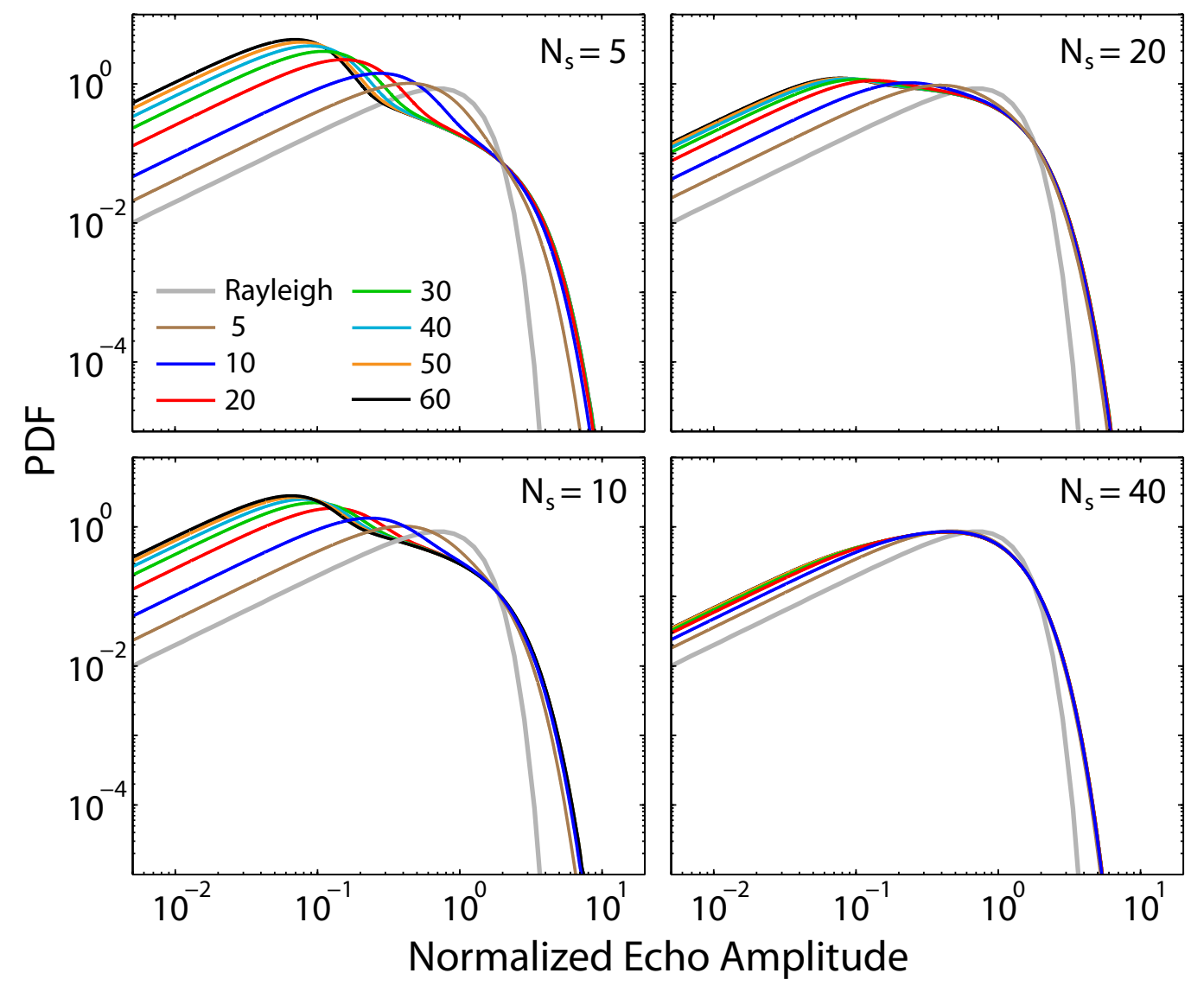

Figure 3-8: Comparison of CF-based mixed assemblage pdf produced with varying $r_{s w}$ (as shown on the legend) and fixed $N_{s} . N_{w}$ is fixed at 100 for all cases. 
for the proportional factor, $w_{s}$, is also an inherent problem of the mixture models in the context of mixed assemblages, due to the mismatch in the spatial distribution of scatterers (Fig. 3-1). In the Rayleigh mixture case, it is likely that the fit of echo pdf shape can be improved by using more than two components in the mixture model through (3.1) (Gallaudet and de Moustier, 2003). However, with simulated data generated from only two types of scatterers, any extra components would lack physical meaning and do not connect with the underlying scattering processes.

Results of this parameter inference study using simulated data show: 1) although each type of model pdf could be made to fit the simulated data over some, if not all of the conditions, the accuracy of the inferred parameters varies dramatically, depending on which model pdf is used and which range of parameters is studied; 2) the CF-based mixed assemblage pdf is necessary for not only producing a satisfactory fit to the shape of the echo pdfs, but also for accurate inference of mixed assemblage composition over a wide range of parameters. Its ability to infer parameters degrades when the number of dominant scatterers becomes large, and the corresponding changes in pdf shape become small; 3) The 2-component Rayleigh mixture model generally failed to fit the shape of the echo pdf of simulated data, because the Rayleigh distribution is not adequate for describing the scattering from a finite number of scatterers with associated beampattern effects. Furthermore, it produces up to order-of-magnitude errors in the inference of assemblage composition owing to its erroneous assumption on the underlying spatial distribution of different types of scatterers; 4) The 2-component CF-based mixture model is capable of fitting the echo pdf shape of simulated data, but can produce up to order-of-magnitude errors in the inference of assemblage composition, for the same reason given in 3).

It should be noted that although the above analysis is based on normalized echo pdfs, in practice, the absolute scattering levels in un-normalized echo pdfs may provide important information for identifying scatterers and should be considered in the analysis of data collected in field experiments. 


\subsection{Summary and conclusion}

In this paper, a general expression for the echo pdf of mixed assemblages is formulated based on the method of characteristic functions (CFs). By incorporating the beampattern effects from an axisymmetric transceiver aperture, the theoretical pdf curves are validated by numerical simulations and shown to have high accuracy over a wide range of conditions. The CF-based mixed assemblage pdf is observed to be highly non-Rayleigh when the number of the strongest type of scatterer in the assemblage is low and gradually approaches the Rayleigh distribution as the number of the strongest type of scatterer increases.

This new CF-based mixed assemblage pdf significantly outperforms the commonlyused $M$-component mixture model (Rayleigh-based and CF-based) when used as a tool to infer the parameters of simulated two-component mixed assemblages. The 2-component Rayleigh mixture model generally fails to adequately fit the shape of the echo pdf of simulated data. This mixture model and the 2-component CF-based mixture model also both result in up to order-of-magnitude errors in the parameter estimates. In spite of the high accuracy in predicting the echo statistics from mixed assemblages, the usefulness of the CF-based mixed assemblage echo pdf in inferring the assemblage composition from simulated data is limited to cases where the number of dominant scatterers is relatively small. This is due to the fact that changes in the shape of these pdfs owing to changes in number of scatterers is inherently small when the number of scatterers is large.

The results show that, in order to accurately model and analyze the echo statistics

of mixed assemblages, it is important to 1) rigorously account for the scattering from a finite number of scatterers and the associated beampattern effects, and 2) rigorously account for the spatial distribution of the different types of scatterers when combining their scattering contribution to the echo. When the echo data appear to be stationary in the analysis window but the groundtruth information suggests that there is more than one type of scatterer in the aggregation, the CF-based mixed assemblage pdf, instead of the $M$-component mixture model, should be employed. 
Mismatches between model assumptions and the actual scatterer distribution can lead to order-of-magnitude errors in data interpretation.

Although this study is inspired in the context of acoustic studies of mixed biological aggregations in the ocean and the examples given in this paper are specific to volume backscattering sources with an axisymmetric transceiver aperture, the general formulation of the CF-based mixed assemblage pdf is applicable to other types of mixed assemblages in different environments measured with different systems. Application of this new mixed assemblage pdf in the analysis of field measurements may provide a new dimension of information toward classifying and discriminating between various kinds of aggregations in nature through use of radar/sonar systems. 


\section{Chapter 4}

\section{Statistics of broadband echoes: application to estimating numerical density of fish}

\subsection{Introduction}

Recent advancements in high-frequency broadband scattering techniques and instrumentation have revealed new opportunities to develop reliable methods for the interpretation of echoes from biological or physical sources in the ocean (Foote et al., 2005; Lavery et al., 2010b; Roberts and Jaffe, 2008; Stanton et al., 2010, 2012). The broadband capabilities of these systems provide continuous scattering information over a wide frequency range and improve temporal resolution and signal-to-noise ratios through pulse compression processing (Chu and Stanton, 1998; Turin, 1960). The broad spectra of echoes collected in the field have been utilized to classify fish of different size classes based on swimbladder resonances in the low kilohertz region (Stanton et al., 2012) and to distinguish the scattering between zooplankton and oceanic microstructure using scattering spectra over a wider high-frequency band (Lavery et al., 2010b). The fine temporal resolution has also been utilized for direct identification of dominant scattering mechanisms for individual marine organisms (e.g., Au 
and Benoit-Bird, 2008; Lee et al., 2012; Reeder et al., 2004; Stanton et al., 1998b). However, the use of broadband signals in acoustic scattering studies also poses new challenges, such as reliable calibration of broadband transducers (Dragonette et al., 1981; Foote, 2006; Foote et al., 1999a; Stanton and Chu, 2008) and high fluctuations in high-frequency echo spectra.

The statistics of echoes, such as the shape of the probability density function of the magnitudes of complex echo amplitudes (hereafter referred to as "echo pdf"), provide a route for deriving discriminative information from echoes through summarizing echo fluctuations over consecutive insonification of the same target or group of targets (Stanton and Clay, 1986). The characteristics of the echoes vary according to the properties and composition of the sources of scattering, and depend strongly on the degree to which the echoes overlap, which is determined directly by the resolution of system (Abraham and Lyons, 2002, 2004; Chu and Stanton, 2010). In the context of volume scattering, a resolution cell is defined as the intersection between the solid angle subtended by the sonar beam and the temporal resolution of the signals, which is the length of the gated sinusoidal wave in the case of narrowband signals, and approximately the inverse of bandwidth for broadband signals processed using pulse compression. For the scattering boundaries, the size of the resolution cell is associated with the size of the footprint of the sonar beam (Abraham and Lyons, 2004).

When the echoes are from coherent summation of a large number of random scatterers within each resolution cell, the echo pdf converges to the Rayleigh distribution asymptotically as a result of the central limit theorem (Goodman, 1985). Therefore, the degree to which the statistics of echoes deviate from the Rayleigh distribution is often used as a proxy to describe the scattering from an aggregation of scatterers in a scenario different from the above. Due to the potential of noise contamination in low-amplitude echoes, the most reliable echo pdf features are usually observed in the high-amplitude "tail" of the echo pdf, which is the region where the values of the echo amplitude are relatively high and probability densities are relatively low (Jones, 2012; Stanton and Chu, 2010).

The application of echo statistics analysis has been an integral component for 
many remote-sensing research fields, such as in the study of electromagnetic scattering from sea surface using radar and the characterization of acoustic scattering from seafloor features using sonar (e.g., Abraham and Lyons, 2010; Ward et al., 2006; Watts and Wards, 2010). Most of these studies used a data-driven approach in which echo observations are fit using generic probabilistic distributions or their mixtures to determine the best representation of echo statistics features (e.g., Gallaudet and de Moustier, 2003; Gelb et al., 2010; La Cour, 2004). Since these models were derived empirically without explicit connections between model parameters and the underlying sources of scattering, the models are not predictive, and their application is often limited to data collected using similar systems in specific geographical locations. To overcome these problems, a physics-based approach was taken in the derivation of theoretical models in a number of other studies (e.g., Abraham and Lyons, 2002; Crowther, 1980; Middleton, 1999). Results of these studies showed that, by rigorously considering the various components in the acoustic scattering processes, including characteristics of the system, signal, and scattering sources, features of the echo pdfs can be exploited for direct inference of the scatterers or parameters of interest.

The physics-based approach has also be used to study the statistics of echoes from marine organisms. For example, in the case where echoes from a single animal are considered, research has shown the connection between the observed echo pdf features and the dominant scattering mechanisms of the animal at different angles of orientation or behavior states (Clay and Heist, 1984; Stanton et al., 2004). Theoretical models capable of predicting statistical features of the echoes as a function of the properties and composition of the insonified target or target aggregations have also been developed (Chu and Stanton, 2010; Denbigh and Smith, 1991; Jones, 2012; Stanton and Chu, 2010; Wilhelmij and Denbigh, 1984). These models can be used as inference tools to provide complementary information in addition to conventional spectral and temporal echo features for accurate interpretation of echoes for the characterization of scattering sources in a direct-path geometry or under the influence of ocean waveguide. 
However, the echo statistics model mentioned above may be of little use in the analysis of narrowband data collected in the field, primarily due to their use of the assumption of continuous-wave (CW). This is caused by the limited temporal resolution of narrowband signals, which under typical field scenarios would result in significant echo overlap and consequently strong Rayleigh-like echo pdf features. This can be problematic, since the discriminative power of echo statistics analysis relies on the presence of non-Rayleigh features, and the performance of such methods degrades rapidly when the echo pdf approaches the Rayleigh distribution (Denbigh and Smith, 1991). Furthermore, the recent development of broadband echosounders has provided much valuable information that can be explored for better echo interpretation as has been previously mentioned, but the existing narrowband (CW) echo statistics models are incompatible with these new broadband data. Therefore, to take full advantage of the broadband signals and the associated substantially-improved temporal resolution, a physics-based broadband echo pdf model is developed in this chapter and applied to analyze data collected in the field.

There are several outstanding challenges in modeling the statistics of broadband echo envelopes. First, broadband pulse-compressed echoes are temporally-localized, and therefore cannot be modeled using random phasors of infinite time span as has been done under the CW assumption. Instead, broadband echo pdfs are formed by sampling the envelopes of the time series consisting of partially-overlapping echo pulses with varying shapes, and the shapes of the pulses are jointly determined by the spectral content of the signals and the frequency responses of the system and scatterers. An approximate analytical solution for the tail of the echo pdf may be derived using a combination of mixture model and Barakat's method of characteristic functions (Barakat, 1974). However, exact analytical formulation for the echo pdf over the entire amplitude is difficult to obtain (Clifford et al., 1993), compounded by complications associated with system- and scatterer-specific parameters.

A Monte Carlo numerical simulation framework is developed in this chapter to model the pdf of the magnitudes of broadband pulse-compressed echo envelopes. This physics-based modeling framework rigorously accounts for important components in 
the processes by which sound is transmitted, scattered, and received. The numerical model is implemented using the exact parameters of a high-frequency broadband single-beam echosounder in a direct-path geometry typical in acoustic scattering studies of marine organisms (Fig. 4-1). The parameters include the frequency-dependent responses of the sonar system, scatterers, and transducer beampattern, and the model can easily be extended to incorporate addition information derived from groundtruth observations, such as the distribution of the size and angle of orientation of the scatterers. This model is applied as an inference tool to estimate the numerical density of fish in monospecific herring aggregations observed in the Gulf of Maine, and results in density estimations comparable to those obtained from conincident volume backscattering strength $\left(\mathrm{S}_{\mathrm{V}}\right)$ measurements and modeled target strengths (TS). Limitations of the broadband echo pdf model as a tool for echo analysis and potential errors associated with various model assumptions are also discussed.

Although this study is inspired in the context of acoustic scattering study of marine organisms, the model developed here is general and can be applied to predict the echo pdf from other types of scattering sources in different environments observed using different systems, such as the scattering of electromagnetic signals received by radar systems. This generality is a direct benefit of the physics-based approach which allows explicit adjustment of model parameters according to specific experimental conditions and the environment.

This chapter is organized as follows. The numerical modeling framework for broadband echo pdf is introduced and results of model implementation are shown in Sec. 4.2. Sec. 4.3 contains detail of the selection and processing of echo data from the field experiment. In Sec. 4.4, numerical densities of fish are estimated using the broadband echo pdf model and two TS-based methods, and the results are compared. The summary and conclusion of this study are given in Sec. 4.5. 


\subsection{Numerical simulation of broadband echo pdfs}

\subsubsection{Modeling framework for numerical simulation of broad- band echo pdf}

The modeling framework used in this study is developed in the time domain based on the temporally-localized features of broadband pulse-compressed echoes (Fig. 41). In contrast to the echoes of $\mathrm{CW}$ signals that are of infinite length so that all echoes overlap, broadband pulse-compressed echoes are localized in time and will generally, at most, only partially overlap with one another. In this framework, a gate of length $\tau$ is arbitrarily chosen to represent the temporal span of the analysis window in which samples of the echo envelopes are taken. The volume included in this gate is a hemispherical shell in space and the thickness of this shell, $L_{g}$, is related to the temporal length of the gate by

$$
L_{g}=c \tau / 2
$$

where $c$ is the sound speed in the medium. For each independent realization, an echo time-series in the gate is generated from an arbitrary number of randomly-distributed scatterers. Independent samples are taken from the envelopes of repeated realizations to form the ensemble from which the echo pdf is derived. Note that the length of the gate is much larger than the size of the resolution cell (approximately given by the inverse of the signal bandwidth when echoes are pulse-compressed).

Each scatterer in the hemispherical shell is randomly located in space, and the location is determined jointly by its temporal location in the gate, which corresponds to the distance between the scatterer and the system, and its angular position with respect to the axis of the transducer aperture (Fig. 4-1). The temporal location of each scatterer in the gate is generated by drawing a sample from a uniform distribution over a range of $[0, \tau]$, and its angular position is generated by calculating the polar and azimuthal angles of a randomly-located point in a hemispherical half-space.

The various parameters involved in this numerical framework are summarized below in mathematical terms (Fig. 4-2). Assume the ideal (computer generated) trans- 


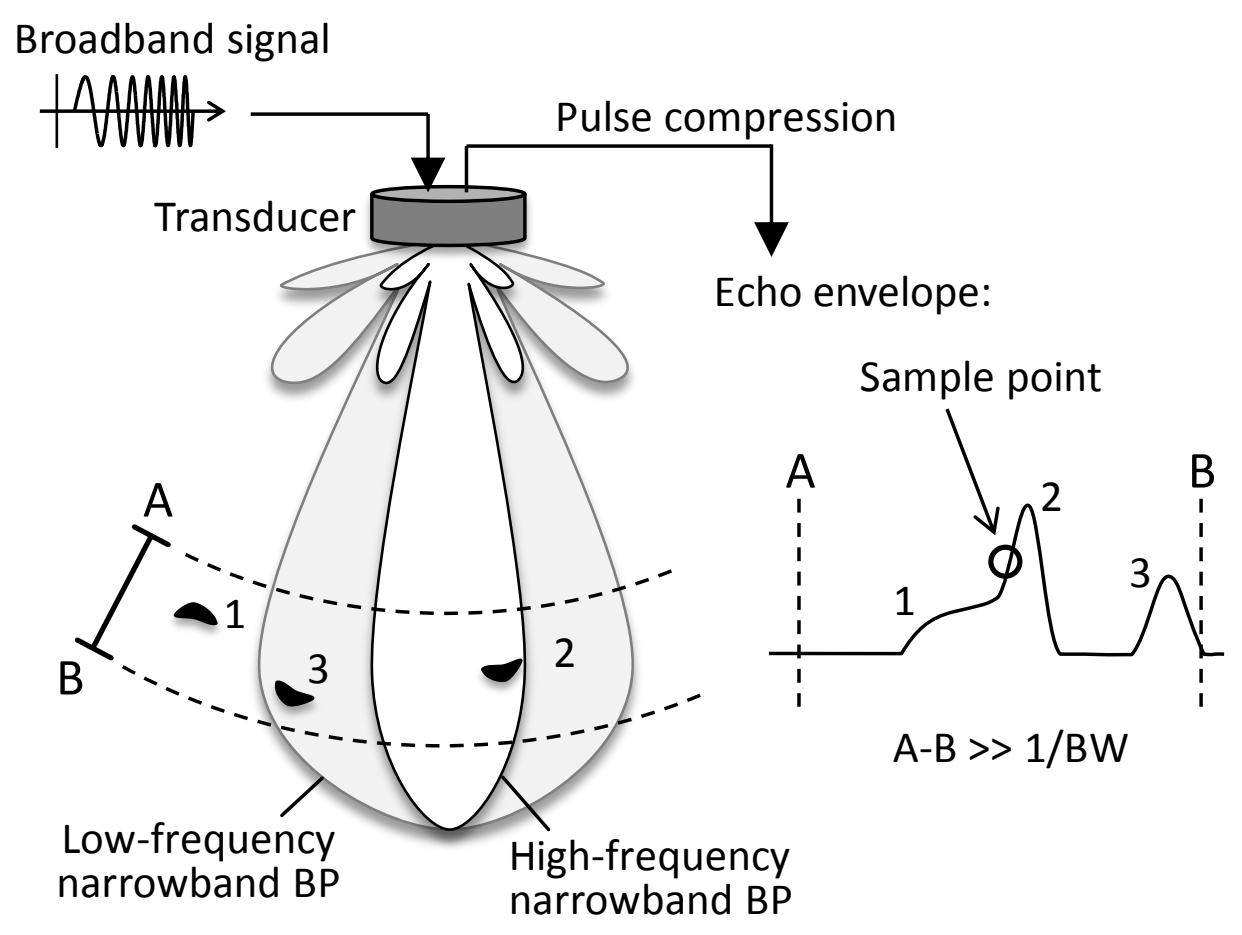

Figure 4-1: A schematic of one realization of the numerical model showing important elements in the modeling framework. The model uses broadband signals and the echoes are processed using pulse compression before the envelopes are taken. The sample point is arbitrarily selected in the region away from the edges of the range gate, $\overline{\mathrm{AB}}\left(=L_{g}\right.$ in text). A transducer with a circular aperture is used here for illustration, and can be substituted according to the specific system parameter. A low-frequency and a high-frequency narrowband beampattern (BP) are sketched to illustrate the frequency-dependent property of the beampattern. Note that the echoes are modulated across all frequencies according to their locations in the beam. Compared to the other two scatterers, scatterer \#2 is located within the mainlobe of the beam and therefore results in a sharper echo (see Sec. 4.2.2). 
mit signal is $y(t)$ and the impulse response of the system (which includes frequencydependent modifications of the transducer and system circuit) is $s(t)$, the actual signal transmitted from the transducer $y_{s}(t)$ is the convolution of the two, given by

$$
y_{s}(t)=y(t) * s(t)
$$

The echo pulse from the $i$ th scatterer is

$$
e_{i}(t)=r_{s s}(t) \otimes h_{i, t o t}(t)
$$

where $r_{s s}(t)$ is the autocorrelation function of the actual transmit signal,

$$
r_{s s}(t)=y_{s}(t) \otimes y_{s}(t)
$$

and $h_{i, t o t}(t)$ is the composite impulse response of the $i$ th scatterer (Chu and Stanton, 1998). The composite impulse response can be expressed as

$$
h_{i, t o t}(t)=h_{\text {scat }, i}(t) * h_{D, i}\left(t, \tau_{i}\right) * h_{B, i}\left(t, \theta_{i}, \phi_{i}\right),
$$

where $h_{\text {scat }, i}(t)$ is the impulse response of the scatterer, $h_{D, i}\left(t, \tau_{i}\right)=\delta\left(t-\tau_{i}\right)$ represents the temporal location of the scatterer in the gate, and $h_{B, i}\left(t, \theta_{i}, \phi_{i}\right)$ is the non-uniform weighting factor imposed by the frequency-dependent beampattern depending on the location of the scatterer in the beam, which is a function of the polar $\left(\theta_{i}\right)$ and azimuthal $\left(\phi_{i}\right)$ angles of the scatterer with respect to the center of the transducer. In this study, the model is implemented with the assumption of a circular aperture with axisymmetrical beams. In this case, the beampattern response depends only on the polar angle of the scatterer and therefore $h_{B, i}\left(t, \theta_{i}, \phi_{i}\right)=h_{B, i}\left(t, \theta_{i}\right)$. Unless otherwise specified, the exact parameters of the high-frequency broadband echosounder described in Sec. 4.3.1 are employed for model implementation in this study. Details of other important components are discussed in Sec. 4.2.2 to Sec. 4.2.4. 
The time-series resulted from the contributions of $N$ arbitrary scatterers is

$$
e^{\Sigma}(t)=\sum_{i}^{N} e_{i}(t),
$$

from which the envelope of the echoes, $a(t)$, is computed and a random sample of the envelope, $a$, is drawn. For the convenience of model implementation, the samples are drawn from a fixed location in the middle of the range gate in this study as depicted in Fig. 4-2. This procedure is equivalent to drawing samples at random locations away from the edges of the range gate, due to the random temporal locationsof the scatterers in the gate. The kernel density method is selected to estimate the echo pdf,

$p_{A}^{\Sigma}(a)$, based on its ability to produce a continuous estimated density function and an intuitive explanation of the smoothness of this function based on signal processing theory (Scott, 1992). To avoid the violation of the boundary condition that $a>0$ and to produce smooth estimation in the high-amplitude tail where the number of samples may be scarce, the envelope samples are log-transformed before the kernel density estimation is performed, and an inverse transformation is used to obtain the final estimated pdf in the linear domain. The algorithm proposed by Botev et al. (2010) is used here for its efficiency and adaptive kernel bandwidth selection capability.

The logarithmic transformation was used in order to obtain a smoother density function in the tail where the occurrence high-amplitude echoes are sporadic, and also to satisfy the constraint that echo amplitudes are always positive. Note $p_{A}^{\Sigma}(a)$ here is analogous to $p_{A}^{\Sigma}(a)$ in Chapter 3 except for the difference between the envelope of broadband and narrowband (CW) signals, and will be referred to "broadband echo pdf model" throughout this chapter.

\subsubsection{Effects of broadband beampattern response}

When scatterers are observed through a directional beam of a sonar/radar transducer, random amplitude modulation factors are imposed on the receiving echoes depending on the locations of the scatterers in the beam, and the resultant echo pdf can be evaluated numerically using the analytical formula in (3.11) (Chu and Stanton, 


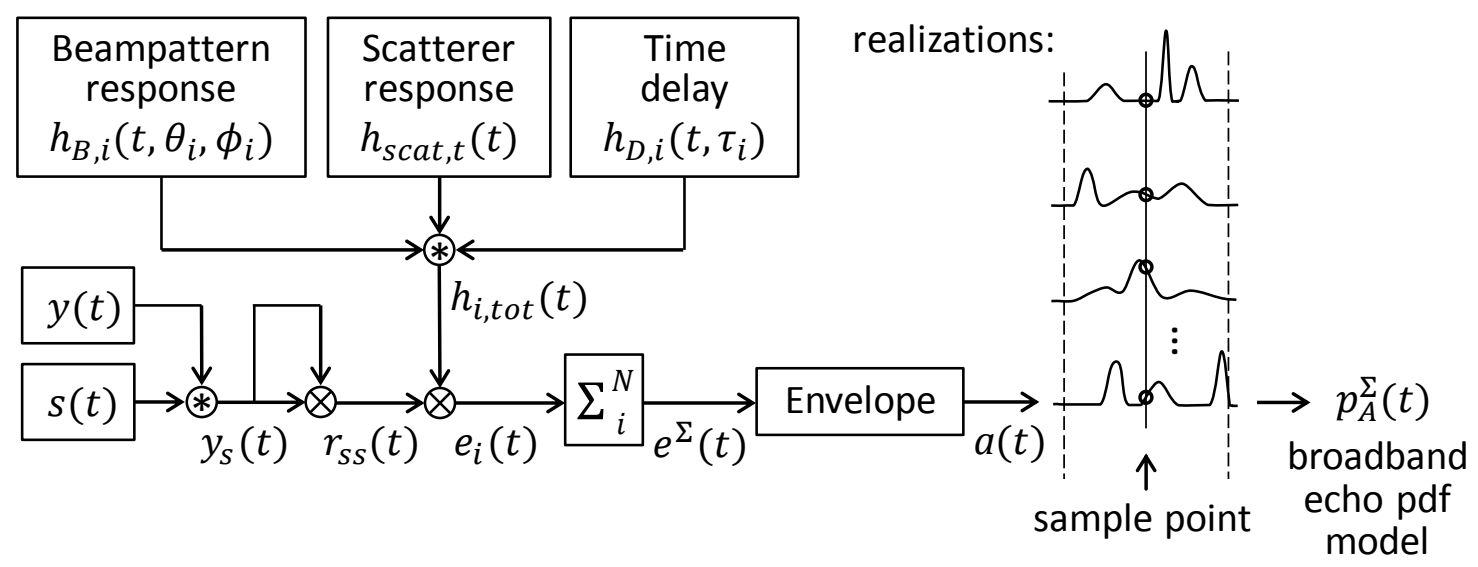

Figure 4-2: Block diagram of the numerical simulation procedure. All involving components are discussed in Sec. 4.2. Note the block diagram depicts the procedure to generate one realization. The ensemble of samples collected from multiple realizations is used for the estimation of the echo pdf model.

2010; Ehrenberg, 1972), involving the probability distribution associated with this echo amplitude modulation (the "beampattern pdf", see Sec. 3.1). For echoes collected using narrowband signals, the resultant echo pdf produced under the influence of the "beampattern effects" is frequency-specific and varies with increasingly heavier tail at higher frequencies (Fig. 4-3). For echoes collected using broadband signals, the sonar/radar beamwidths and associated modulation from the beampattern effects become frequency-dependent and can be described by the beampattern impulse response, $h_{B}(t, \theta)$ [Fig. 4-4(a)]. For scatterers located near the axis of the aperture, the scatterers are included in the mainlobes of the beams for all frequencies, and the frequency response of the beampattern resembles that of an "all-pass" filter. On the other hand, scatterers located further away from the axis of the aperture are only included in the mainlobes of lower frequency beams and fall into the sidelobes of higher frequency beams. In this case, the beampattern filtering is uneven across the frequency and has a response analogous to that of a "low-pass" filter. By the timefrequency reciprocity of Fourier analysis, these characteristics explain the observed changes in the simulated beampattern impulse response, whose time span increases 

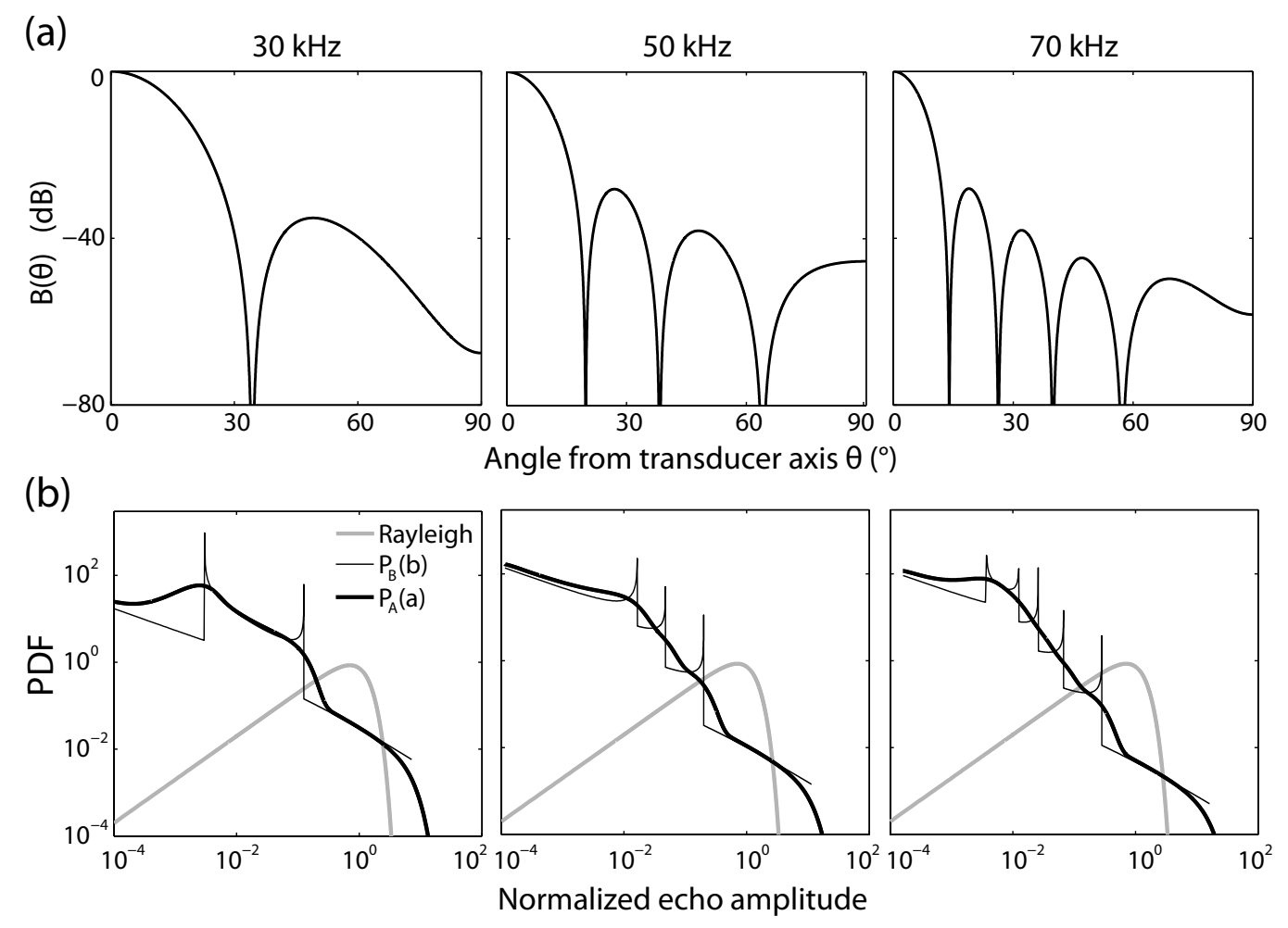

Figure 4-3: (a) Narrowband beampatterns with respect to the polar angle $(\theta)$ of a circular aperture at three frequencies $(30,50$, and $70 \mathrm{kHz})$. The aperture has a radius of $0.054 \mathrm{~m}$, chosen to match the specification of the high-frequency broadband echosounder used in the field experiment (AirMarLow channel, Sec. 4.3.1). (b) Twoway beampattern pdf $\left[\mathrm{P}_{\mathrm{B}}(b)\right.$, thin black line $]$ and the associated echo pdf produced with only one scatterer in the resolution cell $\left[\mathrm{P}_{\mathrm{A}}(a)\right.$, thick black line]. Definition of these quantities can be found in Sec. 3.2.3. Unless otherwise specified, the parameters of the transducer given here are used for all modeling results presented in this chapter.

as a function of increasing polar angle [Fig. 4-4(a)].

Similar to the narrowband cases presented in Chu and Stanton (2010), nonRayleigh echo characteristics are more pronounced for broadband echo pdfs under the influence of the beampattern effects compared to the cases when the beampattern effects are neglected (Fig. 4-5).

Two shaded linear chirp signals are used to investigate the frequency-dependent effects of beampattern on the echo pdfs. The signals are generated by shading an ideal linear chirp signal $(30-70 \mathrm{kHz})$ by two different windows with approximately $20 \mathrm{kHz}$ bandwidths and centered at $40 \mathrm{kHz}$ (LF Hann) and $60 \mathrm{kHz}$ (HF Hann), respectively (Fig. 4-6, first column). These windows are constructed by padding 1's in between 

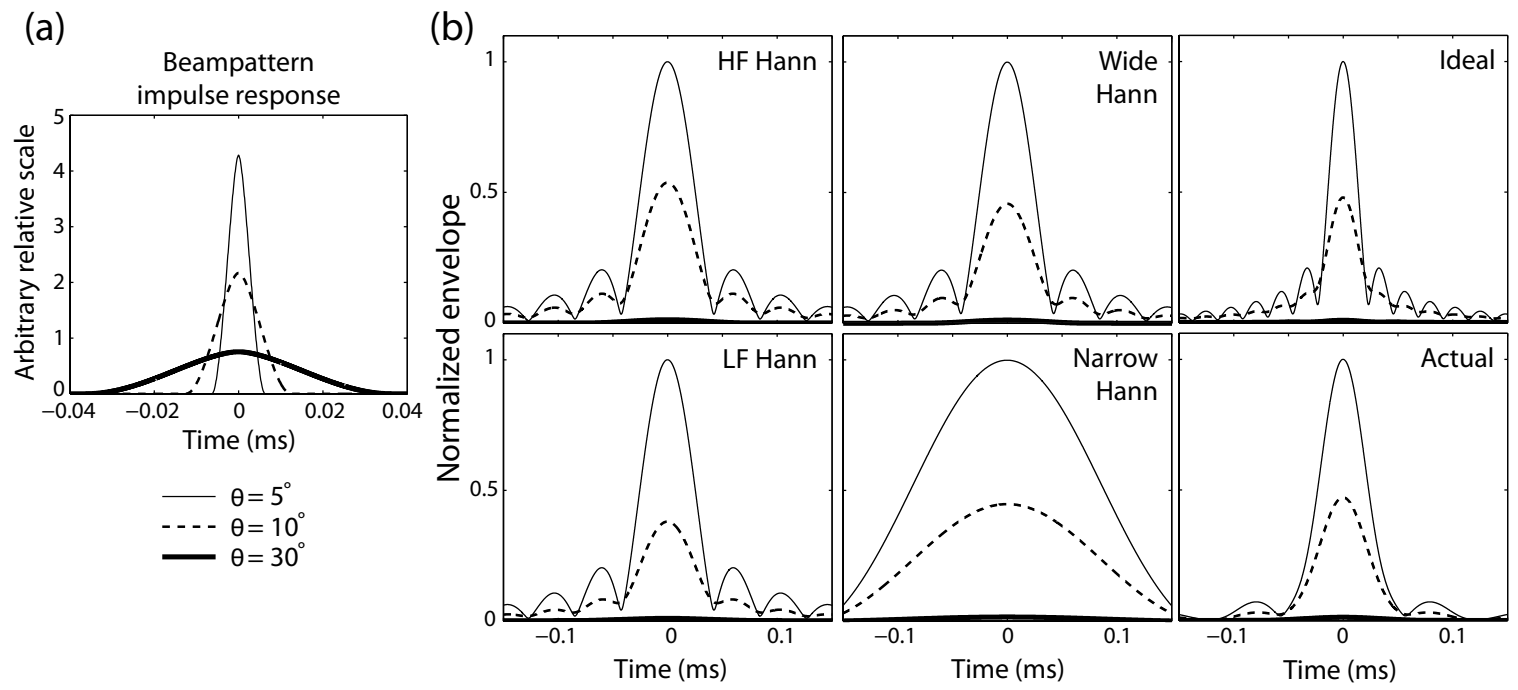

Figure 4-4: (a) The impulse responses of the two-way beampattern at three different polar angles $\left(\theta=5^{\circ}, 10^{\circ}\right.$, and $\left.30^{\circ}\right)$. (b) Time domain characteristics of the autocorrelation functions of different signals modified by the beampattern impulse response. The widths of the responses are jointly determined by the bandwidths and frequency contents of the signals.

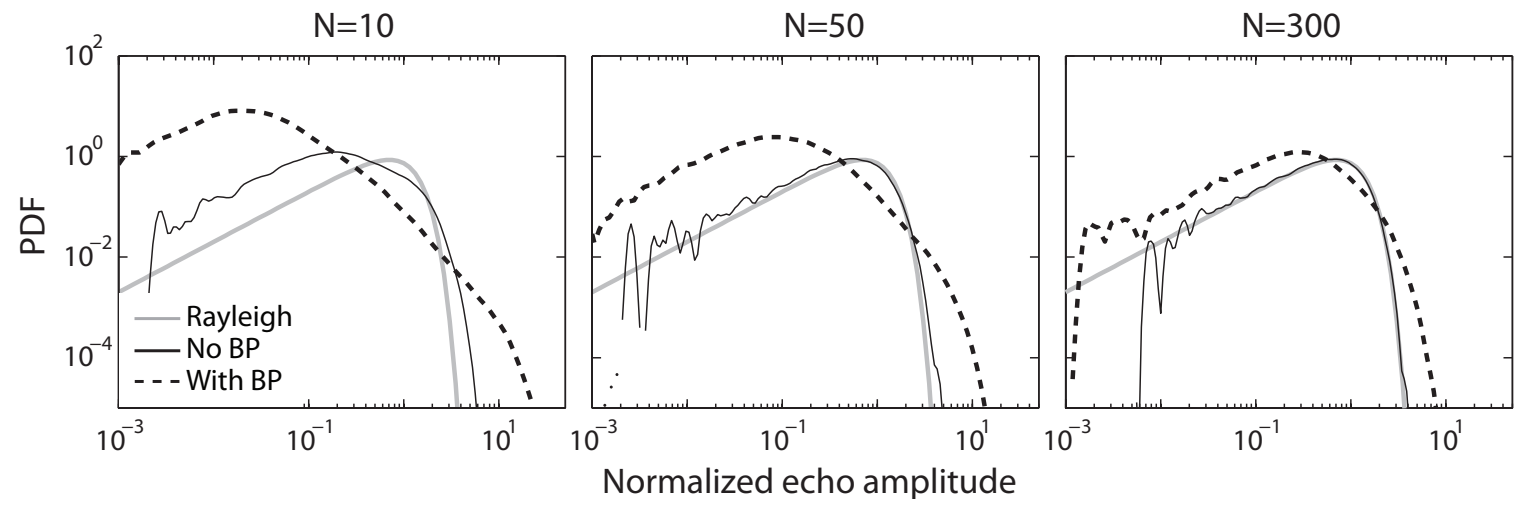

Figure 4-5: Broadband echo pdf models generated with and without the beampattern effects in three cases with different number of scatterers $(N)$ in the range gate. This comparison shows the strong non-Rayleigh influence of the beampattern effects. The Rayleigh distribution is plotted as a background reference for all echo pdf figures in this chapter. Unless otherwise specified, all echo pdfs in this study are normalized according to the procedure in Appendix A. 
halves of a Hann window of a length equals to $1 / 4$ of the transmit signal, and padding 0 's in the front and back of the signal so that the "passband" of the resultant window covers the desired frequencies. These two signals have identical bandwidths and thus the same autocorrelation functions, but the resultant echo pdfs are substantially different owing to the strong influence of the frequency-dependent beampattern effects. Specifically, the narrower mainlobes for the high-frequency components in HF Hann give rise to the heavily-elevated tails in the echo pdfs. From the time domain point of view, the rapid decrease of pulse-compressed echo amplitude for the LF Hann signal compared to that for the HF Hann signal also explains the observed differences in the shape of the echo pdfs (Fig. 4-4(b), first column).

\subsubsection{Effects of signal characteristics}

The influence of signal bandwidth on the echo pdf is investigated in this section using two shaded linear chirp signals. The narrowband signal (Narrow Hann) has a bandwidth of approximately $5 \mathrm{kHz}$ centered at $50 \mathrm{kHz}$, whereas the broadband signal (Wide Hann) has a bandwidth of approximately $20 \mathrm{kHz}$, also centered at $50 \mathrm{kHz}$ (Fig. 4-6, second column). Both signals are shaded using windows constructed by the method described in Sec. 4.2.2. The superior temporal resolution achieved using the Wide Hann signal effectively results in less overlap between pulse-compressed echoes, and therefore produces stronger non-Rayleigh features in the echo pdfs. In addition, the Wide Hann signal contains more high-frequency components than the Narrow Hann signal, which, by the reasons given in Sec. 4.2.2, will also tend to produce elevated tails in the echo pdfs.

However, although the above results can be used to understand the connection between signal characteristics and echo pdf features, the interaction between the temporal and spectral characteristics of the signals and the frequency-dependent beampattern is complicated and requires careful modeling and investigation. Broadband echo pdf models are also computed using two signals related to the use of the

high-frequency broadband echosounder in the field experiment (AirMarLow channel, Sec. 4.3.1). The first signal is the computer-generated ideal transmit signal loaded 

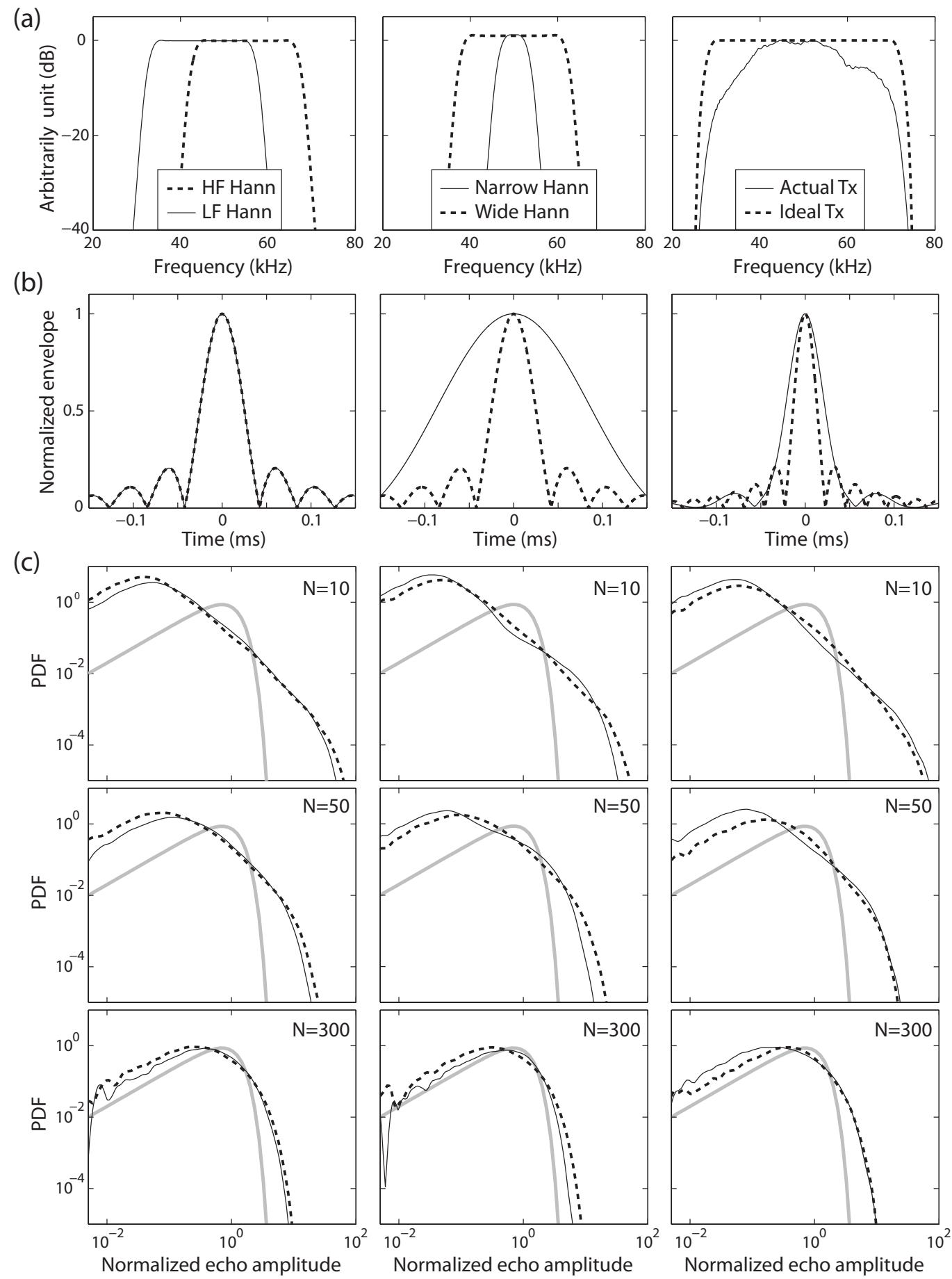

Figure 4-6: Spectra (a), autocorrelation functions (b), and echo pdfs (c) generated using different signals in cases with different number of scatterers in the range gate $(N)$. Comparisons are made between: two linear chirp signals shaded using a narrow Hann window (Narrow Hann) and a wide Hann window (wide Hann); two linear chirp signals shaded using a narrow Hann window (Narrow Hann) and a wide Hann window (wide Hann); and the ideal (Ideal Tx) and actual (Actual Tx) transmit signal associated with the high-frequency broadband echosounder used in the field experiment. 
into the system (Ideal Tx). The second signal is the actual transmit signal (Actual Tx) obtained by modifying the ideal transmit signal using the frequency response of the system measured during calibration (Sec. 4.3.1). Although the system response slightly reduces the bandwidth of the signal, for the cases when the number of scatterer in the range gate is small (e.g., $N=10$ and $N=50$ in Fig. 4-6, third column), the tails are more elevated for echo pdfs computed using the Actual Tx signal. This may be related to the specific tapering in the time domain for the Actual Tx signal [Fig. 4-4(b)]. For cases in which the number of scatterers in the range gate is high (e.g., $N=300$ ), the predicted echo pdfs are very similar in the tail and only differ in the low-echo amplitude portion. This shows that, depending on the numerical density of the scatterers in the experimental scenario, the distinction between different model signals may not be important, since the discriminative power of echo pdf analysis relies primarily on the features of the tails and less on the low-amplitude echoes, which can easily be contaminated by noise.

\subsubsection{Scatterer response}

In this study, the responses of all scatterers are assumed to be uniform across the frequency of interest, i.e., $h_{\text {scat }, i}(f)=h_{\text {scat }, i}$. This assumption is chosen to allow investigation on the influence of other frequency-dependent effects, such as the beampattern and the spectrum of the transmitted signal. The validity of this assumption on fish backscattering data is justified by the understanding that the frequency band of interest $(30-70 \mathrm{kHz})$ is in the geometric regime with weak frequency-dependent features for the scattering from dominant fish species in the survey area, especially in cases where near-normal incident angles dominate the distribution of angles of orientation. The influence of this assumption on the inference results will be discussed in detail in Sec. 4.4.4.

Two probability distributions are prescribed for the distribution of the amplitude of the uniform frequency response. The Rayleigh distribution is first used as the baseline reference to allow direct comparison between the broadband echo pdf models developed here and the narrowband echo pdf models presented in Chu and Stanton 
(2010) and Chapter 3. This choice can be interpreted as a result of the combination of variation of all parameters contributing to the scattering of each scatterer, such as the animal's size, shape, angle of orientation, complex internal structure and outer shape, etc. (Medwin and Clay, 1998). Recall that the Rayleigh distribution is the asymptotic limit of the echo pdf resulted from coherent summation of a large number of independent random phasors (Goodman, 1985).

The second class of models are evaluated assuming the amplitude of the uniform frequency response follows the strongly non-Rayleigh echo pdf generated by a randomly-rough prolate spheroid (Bhatia et al., submitted). This model is chosen because the shapes of elongated marine organisms can be reasonably approximated by randomly-rough prolate spheroids with different aspect ratios, and the echo pdf of squid insonified from all angles of orientation has been successfully fit using this model (Sec. 2.5). Note that the analytical echo pdf model for a randomly-rough prolate spheroid developed by Bhatia et al. (submitted) only considers the situation in which the prolate spheroid is randomly orientation in the plane containing the maximum response axis. To make the modeling scenario compatible with the problem setup in this study, a numerical simulation scheme is employed here to incorporate the scattering from the prolate spheroid in all angles of orientation in the three-dimensional space. Detail of the numerical simulation can be found in Sec. 2.5.2 except for the angle of orientation of the prolate spheroid is allowed to vary randomly in the three-dimensional space here.

Comparison of the broadband echo pdf models computed using the above two amplitude distributions shows the pronounced effect of the non-Rayleigh characteristics of the echoes from individual scatterers on the echo pdf of the aggregation (Fig. 4-7). Note that arbitrary scattering functions can easily be incorporated into the current numerical modeling framework through modification of $h_{\text {scat }, i}(t)$ in (4.5). For the inference of the numerical density of fish in Sec. 4.4, only the Rayleighdistributed uniform frequency response is used to provide a first-order assessment of the performance of the broadband echo pdf model. The influence of other potential characteristics in the response of the scatterers are discussed in detail in Sec. 4.4.4. 


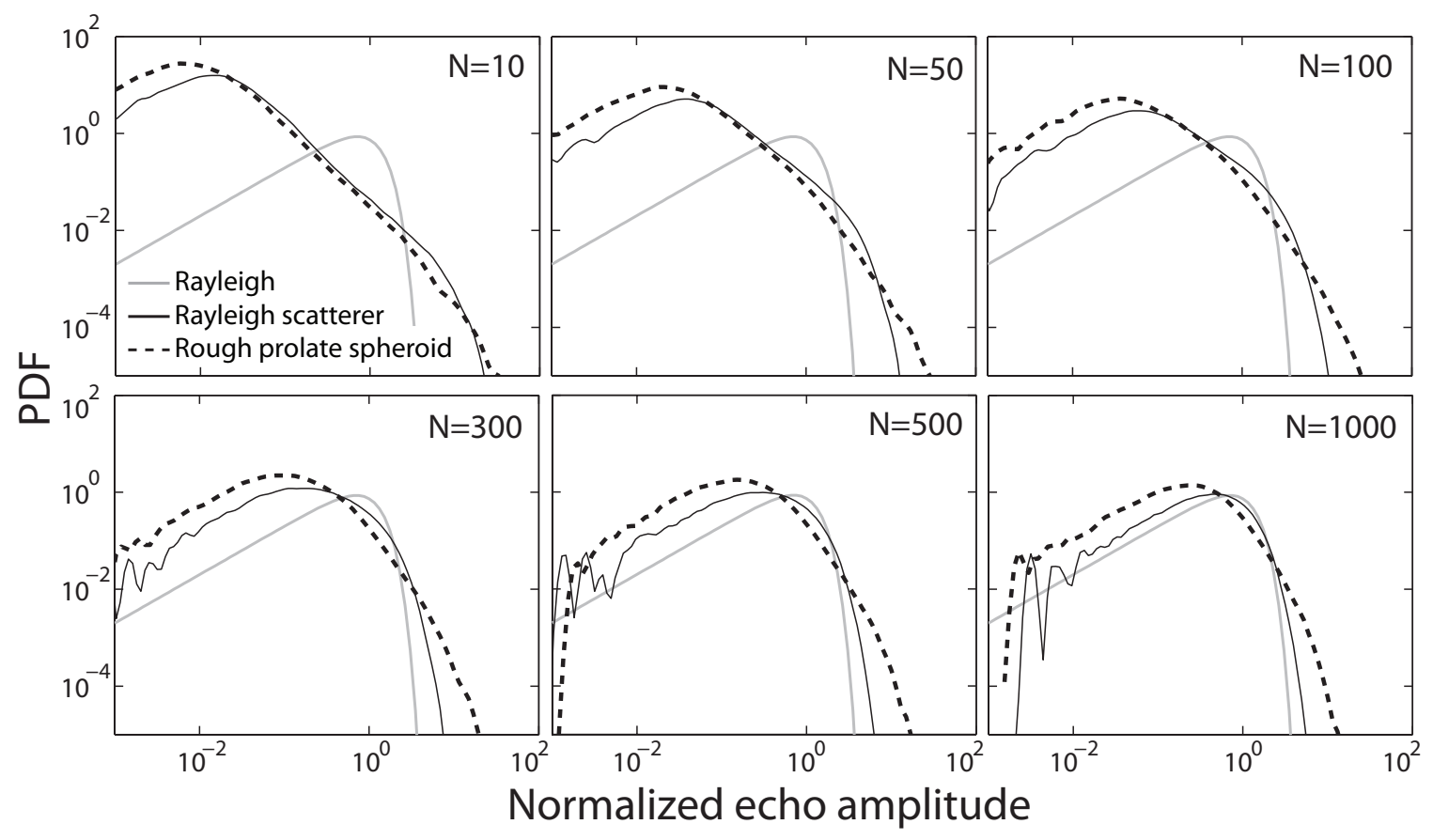

Figure 4-7: Comparison of echo pdf models generated using scatterers with amplitude distributions following the Rayleigh distribution and the distribution of a randomlyrough prolate spheroid (aspect ratio $\xi=5$ ) randomly-oriented in a three-dimensional space. The influence of the strong non-Rayleigh characteristics of the scattering from the rough prolate spheroid is evident in the tail of the resultant echo pdf. 


\subsubsection{Broadband echo pdfs from monotype aggregations and mixed assemblages}

The numerical model is implemented to investigate the statistical characteristics of echoes for both monotype aggregations and mixed assemblages. Following the definition given in Chapter 3, "monotype aggregations" refer to cases in which all scatterers are of the same type, as opposed to in the "mixed assemblages" where there is more than one type of scatterer spatially-interspersed and uniformly-distributed in the analysis window. The same "type" of scatterers refer to scatterers with the same scattering amplitude (or, if it is a random variable, the same amplitude distribution with the same mean amplitude) at the frequency under consideration (see Sec. 3.1 for detailed definition of mixed assemblages). The same set of parameters as in Chapter 3 is used in modeling the echo pdf from mixed assemblages, including the number of scatterer types, the ratio of their backscattering cross sections, and the number of each type of scatterer in the gate. The modeling and analysis are based on two-component mixed assemblages so that basic properties of broadband mixed assemblage echo pdfs can be examined without excessive complexity.

Broadband echo pdfs of mixed assemblages and monotype aggregations are compared (Fig. 4-8). For convenience, $N_{\mathrm{w}, \text { mono }}$ and $N_{\mathrm{w}, \text { mix }}$ will be used to denote the number of scatterers in the range gate for monotype aggregations and the number of weak scatterers in the gate for two-component mixed assemblages, respectively, and $N_{\mathrm{s} \text {,mix }}$ will be used to denote the number of strong scatterers in the gate for two-component mixed assemblages. Comparisons are made between cases with the same number of dominant scatterers, $N_{\text {dom }}$, in the model gate are the same, i.e., $N_{\text {dom }}=N_{\mathrm{w} \text {,mono }}=N_{\mathrm{s} \text {,mix }}$. The results show that the overall shape of the echo pdf of mixed assemblages deviates significantly from that of monotype aggregations over the full range of echo amplitude when the ratio of backscattering cross section between the strong and weak scatterers, $r_{\mathrm{sw}}$, is small (e.g., $\left.r_{\mathrm{sw}}=5\right)$. When $r_{\mathrm{sw}}$ is large and the strong scatterers dominate the scattering field, the shape of the elevated tails of echo pdfs for both cases are very similar and the echo pdfs only differ in the low echo am- 
plitude portion. As expected, the shape of the broadband echo pdfs approaches the Rayleigh distribution asymptotically with increasing number of dominant scatterers. The consequences of these features in an inference context are discussed in Sec. 4.4.4.

\subsection{Broadband acoustic backscattering data from fish aggregations in the ocean}

\subsubsection{Data collection and system calibration}

Acoustic data were collected during a series of fish backscattering experiments using a towed broadband echosounder (EdgeTech) over Georges Bank area in the Gulf of Maine (see Stanton et al., 2012, for detail). The acoustic measurements were accompanied by a pelagic rope trawl deployed by a separate research vessel. The acoustic system contains four broadband channels that jointly span a frequency range from $1 \mathrm{kHz}$ to $130 \mathrm{kHz}$, with some gaps. The net catches by the trawl provided important groundtruth information on the species composition of the observed fish aggregations as well as the length distribution of each species of fish [Fig. 4-9, also see Fig. 4 in Stanton et al. (2012)].

This study makes use of the echo data from the lower three broadband channels with frequency spans of 1-6 kHz (Shamu), 10-18 kHz (424), and 30-70 kHz (AirMarLow). Data from all three channels are used for spectral analysis, whereas only data from the AirMarLow channel are used for echo statstics analysis. The system was calibrated at various operational depths using a standard spherical target (a $30 \mathrm{~cm}$ diameter Aluminum sphere) (Fig. 4-10). The Shamu channel was calibrated using the "full-wave" approach in which the calibration curve is obtained by comparing the echoes from the entire standard target to theoretical predictions across the frequency band of interest. This method is suitable for the Shamu channel since there are no spectral nulls in the scattering of the standard sphere within this frequency band. The 424 and AirMarLow channels were calibrated using the "partial-wave" approach which takes advantage of the pulse-compression processing and compares 


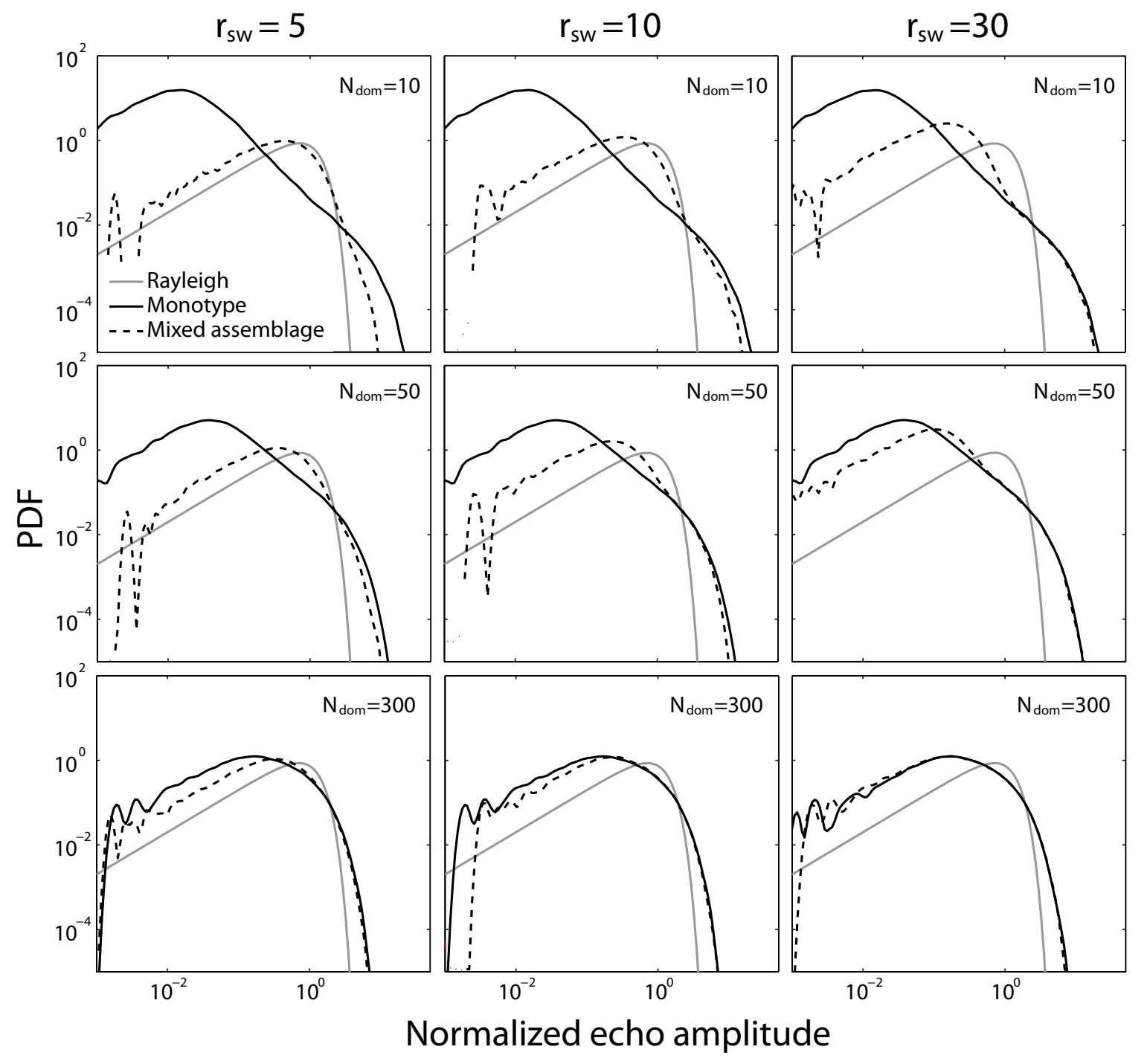

Figure 4-8: Comparison of echo pdf models for monotype aggregation and twocomponent mixed assemblages with varying composition. The ratio between the backscattering cross section of the strong to the weak scatterers in the mixed assemblage $\left(r_{\mathrm{sw}}\right)$ is varied from 5 to 30 . The number of dominant scatterers in the range gate $\left(N_{\text {dom }}\right)$ is defined to be equal to the number of scatterers in monotype aggregations and the number of strong scatterers in mixed assemblage, i.e., $N_{\text {dom }}=N_{\mathrm{w}, \text { mono }}=N_{\mathrm{s}, \text { mix }}$. 


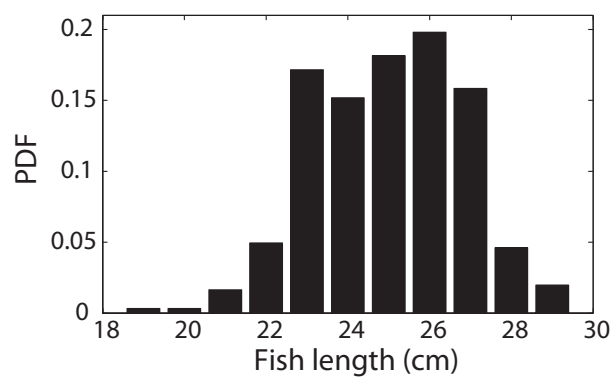

Figure 4-9: Distribution of the length of herring concluded from trawl net catches [reproduced from Fig. 4(c) of Stanton et al. (2012)].

the data and model predictions for the echoes from only the front interface of the target (Stanton and Chu, 2008). This approach was developed with an aim to reduce calibration errors associated with the resonances of the standard target, where uncertainties in the size and material properties of the target that can cause significant variation of the locations of resonances. Full-wave calibration was also conducted for the 424 and AirMarLow channel for comparison purposes [Fig. 4-10(c)]. The full-wave calibration curve corresponds well to the partial-wave calibration for the AirMarLow channel, but larger differences are seen in the low frequency region $(5-7 \mathrm{kHz})$ for the 424 channel. The discrepancy is likely caused by the difficulties in separating the specular reflection from the front interface of the sphere due to the limited bandwidth of the 424 channel [Fig. 4-10(b)]. Detail of the calibration procedures can be found in Stanton and Chu (2008) and Stanton et al. (2010).

\subsubsection{Echo data selection}

A subset of echo data is carefully selected in order to facilitate the assessment of the performance of the broadband echo pdf model as an inference tool for estimating the numerical density of fish. The criteria for the selection of suitable fish aggregations are discussed in this section.

First of all, preference is given to echo data that produce strong non-Rayleigh echo pdfs with elevated tails. This is due to the reasons discussed in Sec. 4.1 that the discriminative power of echo statistics analysis diminishes when the probability distribution of echoes approaches the Rayleigh distribution, which is the asymptotic 

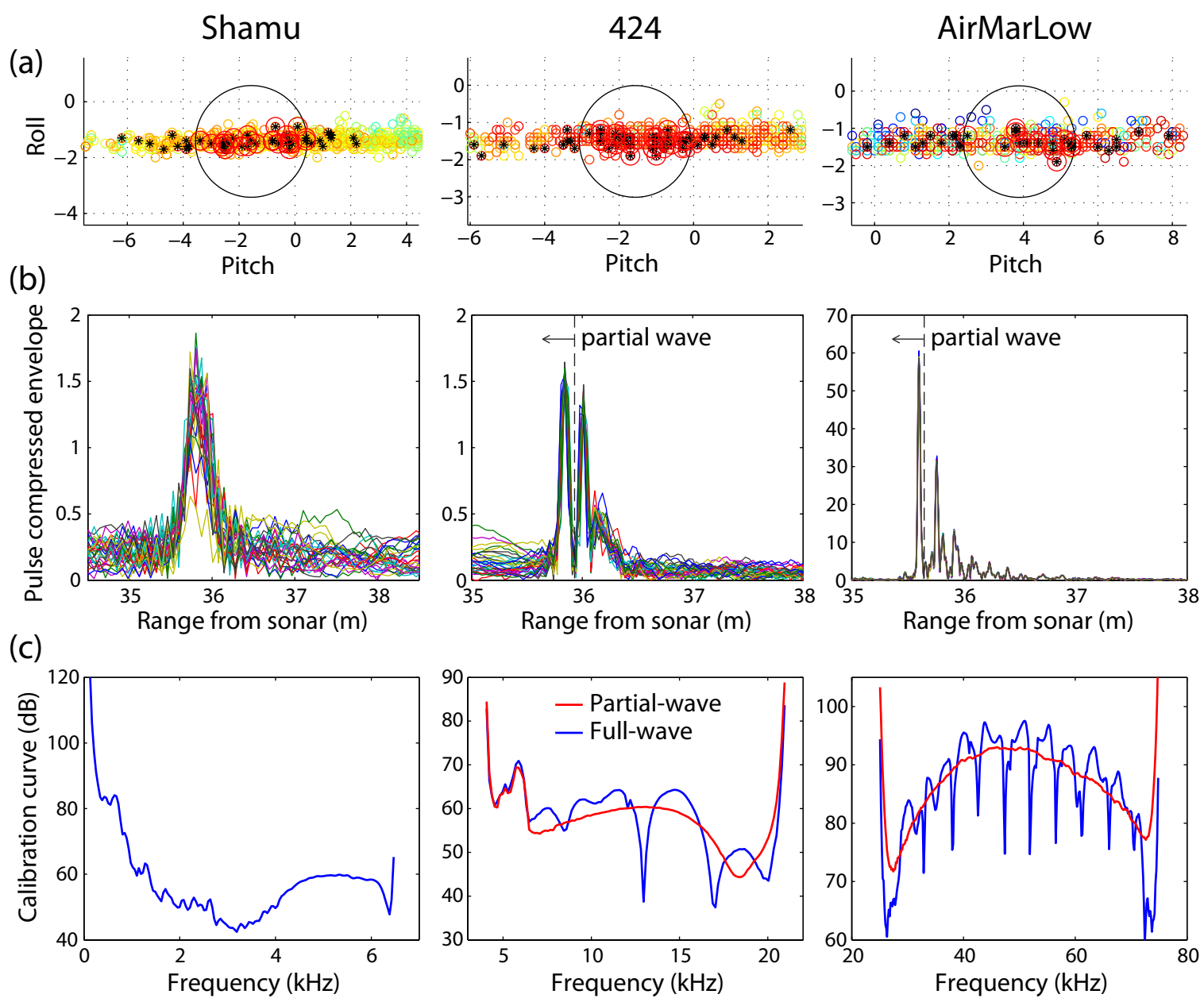

Figure 4-10: Calibration data and results for the three broadband channels (Shamu: 1-6 kHz, 424: $10-18 \mathrm{kHz}$, and AirMarLow: $30-70 \mathrm{kHz}$ ) channels in the EdgeTech broadband echosounder. (a) Sphere echoes selected for use in the calibration. Each echo is plotted as a circle $(\bigcirc)$ according to the roll and pitch angles of the towbody recorded at the instant of sonar transmission. The circles are color-coded according to the relative amplitudes of the peaks of the echo envelopes, with the red-to-blue variation denotes high-to-low amplitude variation. Circles marked with ' $*$ ' are echoes with the top $5 \%$ highest amplitudes. Echoes within an arbitrarily-chosen $1.5^{\circ}$ radius from the mean roll and pitch angles of these marked echoes (further marked by larger red circles) are selected for use in the calibration. This procedure is employed to exclude echoes resulted from off-axis insonification of the standard sphere. (b) Timeseries of the envelopes of the selected echoes. Echoes from the 424 and AirMarLow channel are adjusted so that the peaks of the envelopes of specular reflections are aligned to facilitate paritial-wave analysis. Echoes from the Shamu channel are not adjusted. (c) Results of calibration for all three channels. Results for both the fullwave and partial-wave analyses are shown for the 424 and AirMarLow channel. 
limit of the echo pdfs resulted from coherent summation of a large number of random scatterers. In this study, since samples are taken from rectangular analysis windows on the echogram encompassing a range of depth and a number of pings, fish "patches" [as defined in Stanton et al. (2010)] observed at closer ranges from the echosounder system is preferred. This is because an analysis window at a closer range encloses a smaller volume compared to a window of the same size at a greater range from the sonar. For a constant numerical density of scatterers in space, an analysis window at a closer range would contain a smaller number of fish in the range gate (Fig. 4-1), which results in less overlap among echoes and a higher probability of non-Rayleigh echo pdf.

Second, echo data from patches formed by only one species of fish (a "monospecific" aggregation) is preferred in order to constrain the number of free parameters in the inference problem. For example, for proper parameterization of a mixed assemblage of fish, parameters such as the number of fish species and the ratio of backscattering cross section between species, as well as the numerical density and the distribution of size and angle of orientation for each species, have to be included, and the number of parameters grow significantly as a function of the number of fish species. By constraining the analysis to monospecific aggregations and using the distribution of fish length derived from trawl net catches (Fig. 4-9), the number of model parameters is kept at minimum, which avoids the problem of overfitting and at the same time reduces the computational loads for echo pdf model generation.

Preference is also given to echo data collected from survey periods during which the towbody maintained relatively constant pitch and roll angles while hovering above the fish aggregations. This criterion minimizes the variability in the echo data resulted from changes in the relative orientation and distance between the insonified fish and the system.

Using the above criteria, analysis windows are selected from the interior of a fish patch occurring near seafloor in early daylight hours (approximately two hours after first light) [Fig. 4-11(a)]. The dominant fish species in these aggregations was identified to be Atlantic herring (Clupea harengus) based on a combination of information 
from the observed resonance in volume backscattering strength in the Shamu channel [Fig. 4-11(b)], prior knowledge of the surveyed region, and trawl catches (Nero et al., 2004; Stanton et al., 2010, 2012). The echo pdfs derived from data in the AirMarLow channel contain non-Rayleigh features that can explored for inference through echo statistics analysis Fig. 4-11(c)]. The procedure through which analysis windows are selected and echo pdfs are derived from the acoustic data is discussed in Sec. 4.3.3.

Another set of analysis windows are also selected from a patch of mixed assemblage of fish occurring near the sea surface at night [Fig. 4-12(a)]. The swimbladder resonances observed in this patch have been analyzed in detail by (Stanton et al., 2012). Two prominent resonance peaks are observed in the volume backscattering strength in the Shamu channel [Fig. 4-12(b)]. The echo pdfs from this mixed assemblage derived from data in the AirMarLow channel show very strong non-Rayleigh characteristics with heavily-elevated tails [Fig. 4-12(c)], which likely result from occasional insonification of large fish within a background of an aggregation of small fish. Due to the complexity involved in developing efficient and adaptive inference framework under the constraint of computational capability, echo pdfs from this mixed assemblage are shown but not analyzed (see Sec. 5.2.2 for more discussion).

\subsubsection{Echo pdf from fish aggregations}

Echo pdfs are derived using the acoustic data collected from the AirMarLow channel, due to its narrower beamwidth and broader bandwidth (and thus better temporal resolution) that are more likely to give rise to echoes with strong non-Rayleigh characteristics suitable for echo statistics analysis. Each echo envelope sample is normalized to the rms echo amplitude of all samples in the analysis window (Appendix A). The echo pdfs are estimated using the same procedure outlined in Sec. 4.2.1.

Each echo pdf is derived using all echo envelope samples included in a given analysis window, such as the ones shown in Fig. 4-11 and Fig. 4-12. The sizes of the analysis windows are chosen based on two competing criteria: 1) the analysis window has to to large enough to include enough number of independent echo samples that form a representative ensemble for the estimation of the echo pdf, and 2) the size of 


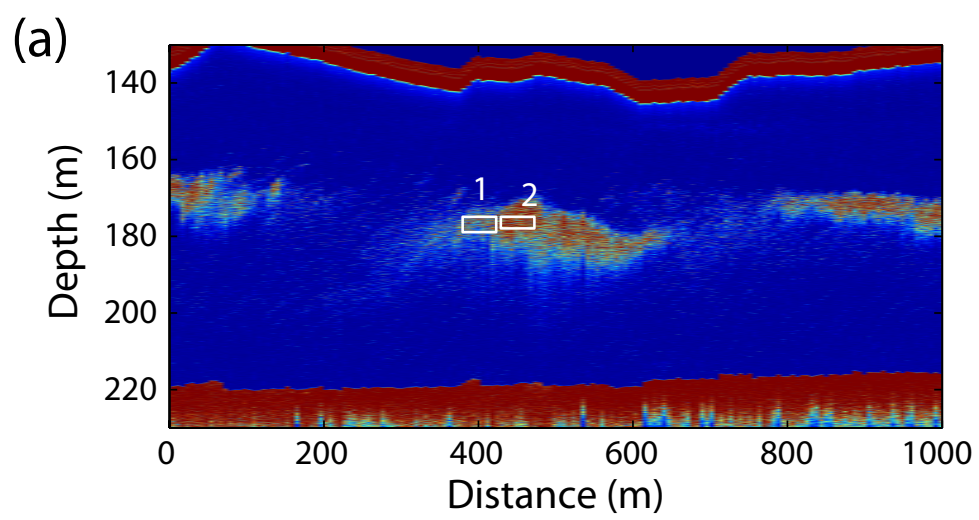

(b)

Box 1
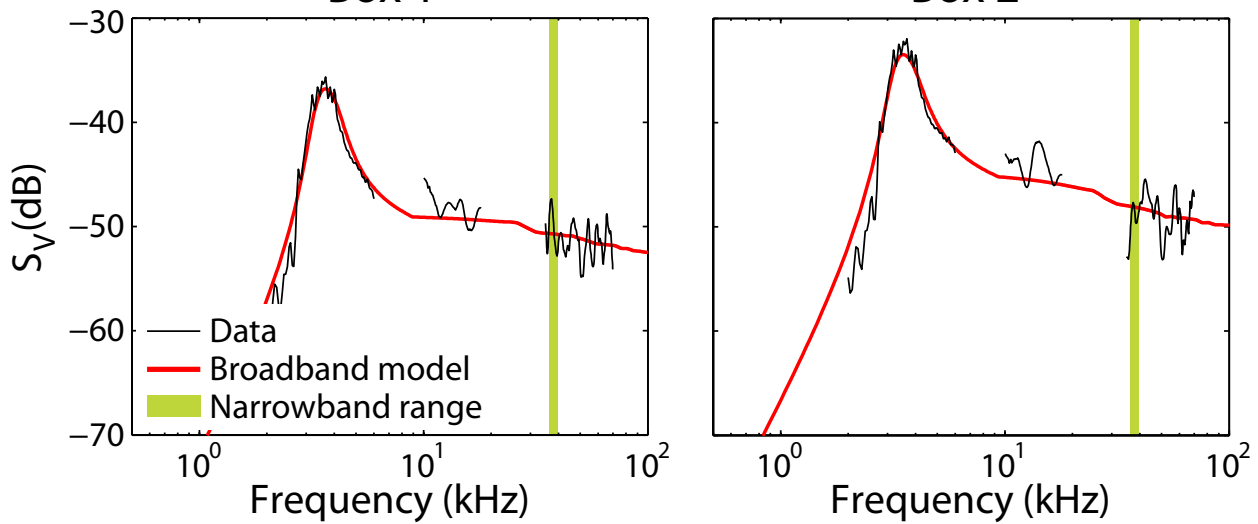

(c)

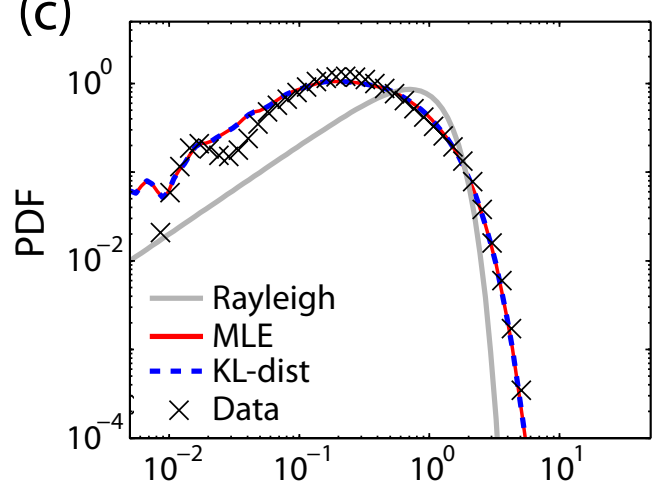

Normalzied echo amplitude

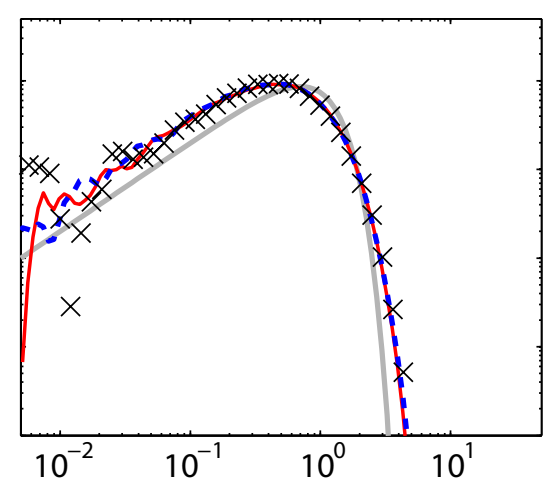

Normalized echo amplitude

Figure 4-11: Examples of data and analysis of monotype (monospecific) aggregations of herring observed near the seafloor in the day time. (a) Echogram of the aggregation with two analysis windows. (b) Broadband volume backscattering strengths $\left(\mathrm{S}_{\mathrm{V}}\right)$ and their respective best-fitting broadband physics-based hybrid models for the two analysis windows marked in (a) (Sec. 4.4.2). Also shown are the ranges of frequency $(36.3-39.7 \mathrm{kHz})$ included for the analysis of narrowband $S_{V}$. (c) Broadband echo pdfs of the data and best-fitting models(Sec. 4.4.1). 

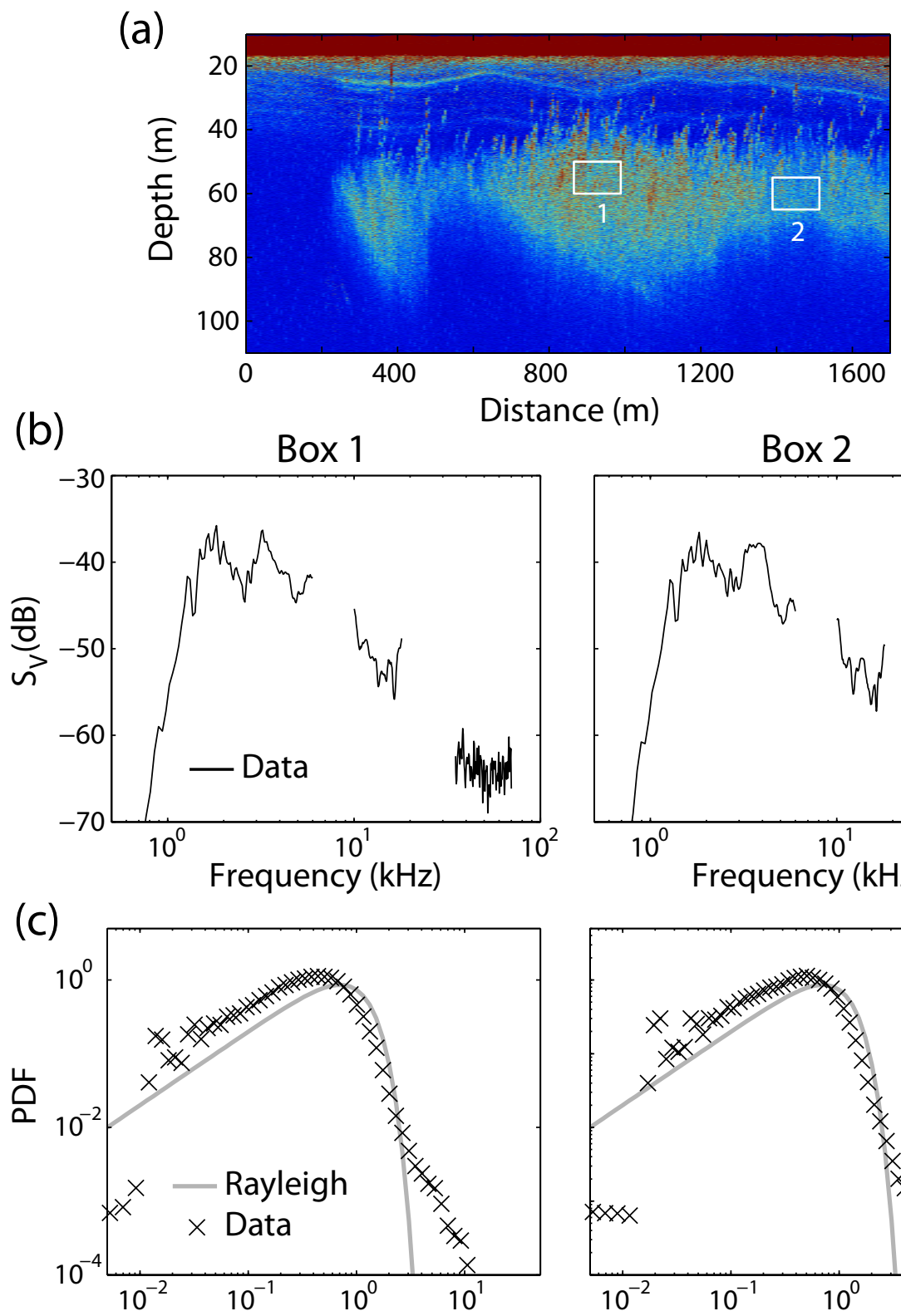

Normalized echo amplitude Normalized echo amplitude

Figure 4-12: Examples of data and analysis of mixed assemblages of fish observed near the sea surface at night. (a) Echogram of the mixed assemblage with two analysis windows. (b) Broadband volume backscattering strengths $\left(\mathrm{S}_{\mathrm{V}}\right)$ for the two analysis windows marked in (a). (c) Broadband echo pdfs of the data. Note the prominent highly-elevated tails that may have been caused by occasional insonification of large fish in the mixed assemblage. This aggregation is the same as aggregation E that has been analyzed in Stanton et al. (2012). 
the analysis window has to be small enough that the scattering processes within the analysis window is stationary within the fish patch (Stanton and Chu, 2010). The second criterion is particularly important for the selection of analysis window within the inherently patchy aggregations of marine organisms. In this study, the above criteria resulted in analysis windows that span a minimum depth range of $4 \mathrm{~m}$ and cover at least 5 pings, which generally include several thousands of envelope samples recorded at the Nyquist sampling frequency during the field experiment.

Since the data were collected without knowledge of the spatial distribution of the scatterers, the response from the same scatterer or the same set of scatterers may be sampled at multiple consecutive temporal locations in the time-series, i.e., adjacent echo samples from the echo time-series may not be statistically independent. However, since these repeated samples can be considered as analogous to oversampling in the temporal domain, the estimation of echo pdf is still possible provided that enough independent samples are included in the ensemble. Owing to the fine temporal resolution achieved through pulse compression processing of broadband signals, some of the envelope samples in the same ping are from independent set of scatterers separated in time by greater than the inverse of the bandwidth. When data across several pings at the same range are considered, the echoes are either 1) from nonconsecutive pings separated by a distance greater than the footprint of the mainlobe of the beam, which ensures that completely different sets of scatterers are insonified, or 2) from consecutive pings sampling different sets of scatterers that partially-overlap but with random phases uniformly distributed over $[0,2 \pi)$. Combining the above, the total number of independent samples included within each analysis window is in the lower hundreds to well over a thousand, depending on the window size. Note that the attenuation of acoustic energy by seawater is negligible within the window at the range under consideration (approximately $40 \mathrm{~m}$ for the windows shown in Figs. 4-11 and Fig. 4-12), and therefore envelope samples within an analysis window at different ranges can be pooled for the estimation of the echo pdf.

By pooling all echo samples included in an analysis window for the estimation of a single echo pdf, the composition of fish aggregation is assumed to be stationary 
within the enclosed volume. More specifically, ping-to-ping variations are assumed to have been produced solely by changes in the scatterer locations in the beam, angle of orientation of each scatterer with respect to the transducer, and distance between each scatterer and the system. Even though the set of insonified scatterers may change across consecutive pings in the analysis window as a result of horizontal movement of the sonar system, the assumption is that the composition of the set of insonified scatterers at each ping, such as the numerical density and the mean scattering amplitude of the scatterers, is identical. The analysis windows in the study are visually selected based on the structure of the observed aggregations revealed through the distribution of echo amplitudes on the echograms. Although formal statistical analysis on stationarity within the analysis windows may be possible using methods that have been developed recently (Borgnat et al., 2010), this is outside the scope of this study and is recommended as a future research topic in Sec. 5.2.3.

\subsection{Estimation of the numerical density of fish in monospecific aggregations}

In this section, the broadband echo pdf model is used as an inference tool to estimate the numerical density of fish in the selected monospecific fish aggregation. The results are compared with the densities estimated using conventional echo integration techniques in combination with a semi-empirical narrowband TS model and a broadband physics-based hybrid model. This comparison provides an assessment of the applicability and performance of the broadband echo pdf model in the analysis of data collected in the field.

\subsubsection{Numerical density estimation using broadband echo pdf models}

A set of broadband echo pdf models are constructed using the exact parameters of the

high-frequency broadband transducer (AirMarLow) in the EdgeTech system. Since 


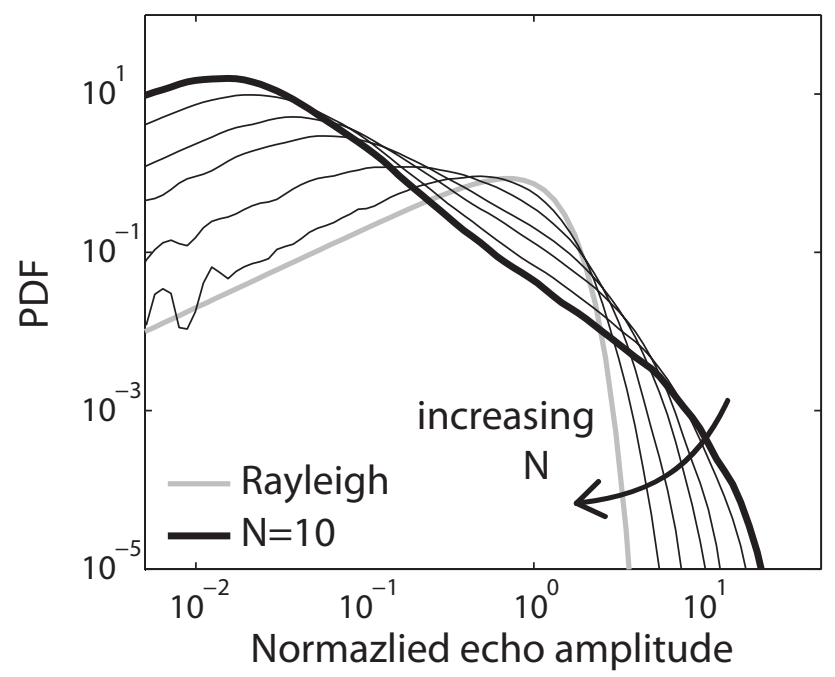

Figure 4-13: Examples of monotype broadband echo pdf model used in the inference analysis. The shape of the echo pdf varies from highly non-Rayleigh toward the Rayleigh distribution with increasing number of scatterers in the range gate $(N)$. The echo pdf model's shown here are produced with $N=10,50,100,200,500$, and 800. Rayleigh-distributed noise was added to each model following the procedure described in Appendix B.

the goal is to infer the numerical density of fish in monospecific aggregations, the only varying parameter in the models is the number of scatterers within the gate (Fig. 413). Rayleigh-distributed noise is added to the ensemble of model realizations before model echo pdfs are estimated. This is necessary to make the models applicable to data collected in the field where noise is present and may influence the statistical properties of the echoes. The procedure of noise addition is provided in Appendix B. The parameters in model implementation are summarized in the paragraphs below.

The modeling scenario assumes an aggregation of $N$ identical scatterers insonified by a transducer with a circular aperture (Fig. 4-1). The aperture has a radius of 0.054 $m$ that corresponds to the dimension of the AirMarLow transducer. The actual transmit signal $\left(y_{s}(t)\right)$ is modeled by modifying the computer generated ideal linear chirp signal $(y(t))$ with the system response $(s(t))$ measured during calibration (Sec. 4.2.3). The response of each scatterer is assumed to be uniform across the frequency band with a Rayleigh-distributed amplitude (Sec. 4.2.4). The length of the gate in which the scatterers are randomly distributed is arbitrary chosen to be $L_{g}=0.5 \mathrm{~m}$. The numerical density of scatterers associated with each model echo pdf can be calculated 
by

$$
\rho \approx N /\left[\frac{2}{3} \pi r^{2} L_{g}\right]
$$

where the term in the bracket represents the hemispherical shell enclosed by the analysis window (Fig. 4-1), and $r$ is the distance between the center of this sampling volume and the sonar system.

Two methods are used to fit the echo pdf derived from the data to the broadband echo pdf models. The first method performs curve-fitting by minimizing the KullbackLeibler (KL) divergence (Kullback, 1959) between the data and the echo pdf models

$$
\mathrm{D}_{\mathrm{KL}}(\rho)=\int p_{\text {model }}(x ; \rho) \log \frac{p_{\text {model }}(x ; \rho)}{p_{\text {data }}(x)} d x
$$

where $\rho$ is the numerical density of scatterers associated with each echo pdf model, and $p_{\text {model }}(x ; \rho)$ and $p_{\text {data }}(x)$ are the echo pdfs of the model and data, respectively. The numerical density is estimated by

$$
\hat{\rho}_{\mathrm{KL}}=\underset{\rho}{\arg \min } \mathrm{D}_{\mathrm{KL}}(\rho) .
$$

Since the calculation of KL divergence depends on the exact values of the pdfs, uncertainties and bias associated with the estimation of echo pdfs (Scott, 1992) can lead to errors in the inference. This problem is addressed by verifying the best-fitting model with the nonparametric two-sample Kolmogorov-Smirnov (KS) test which examines the hypothesis that the data samples and numerical simulations are drawn from the same distribution (Conover, 1999). By comparing the data and all models using the KS test, a confidence interval can also be identified in the model parameter space. In this study, the range of numerical densities associated with echo pdf models that pass the KS test are reported as an estimate of the confidence interval. However, it should be noted that the current resolution of model parameters may be too coarse to give a proper estimate of the confidence interval. Numerical models with more finely-spaced variation of the numerical density of scatterers and the theoretical distribution of Kolmogorov-Smirnov statistic can be used to achieve a better estimation 
of the confidence interval.

The second method uses a maximum-likelihood estimator (MLE) to estimate the numerical density of fish. Assume $x_{1}, x_{2}, \ldots, x_{n}$ are independent and identicallydistributed echo samples included in the analysis window, the method computes the log-likelihood

$$
\begin{aligned}
l\left(\rho \mid x_{1}, x_{2}, \ldots, x_{n}\right) & =\ln \mathcal{L}\left(\rho \mid x_{1}, x_{2}, \ldots, x_{n}\right) \\
& =\ln p_{\text {model }}\left(x_{1}, x_{2}, \ldots, x_{n} \mid \rho\right) \\
& =\ln \prod_{i}^{n} p_{\text {model }}\left(x_{i} \mid \rho\right) \\
& =\sum_{i}^{n} \ln p_{\text {model }}\left(x_{i} \mid \rho\right),
\end{aligned}
$$

and performs the inference by maximizing the log-likelihood

$$
\hat{\rho}_{\mathrm{MLE}}=\underset{\rho}{\arg \max } l\left(\rho \mid x_{1}, x_{2}, \ldots, x_{n}\right) .
$$

The MLE assumes knowledge of the true echo pdf models but does not require the echo pdf of the data to be explicitly estimated. This is advantageous in this study, since the number of echo samples is usually limited for data collected in the field (Sec. 4.3.2), but the size of the ensemble of simulations can be increased to reduce the uncertainties associated with the estimation of echo pdf models. Following the method outlined in Azzalini (1996), a cubic spline is used to interpolate the loglikelihood function and a confidence interval can be determined using

$$
\left\{\rho: 2 l\left(\left(\hat{\rho}_{\mathrm{MLE}}\right)-l(\rho)\right)<c_{\alpha}\right\}
$$

where $c_{\alpha}$ is the (1- $\left.\alpha\right)$-level quantile of $\chi_{k}^{2}$ distribution with $k$ equals the dimension of the parameter space ( $k=1$ in this study), which is the asymptotic distribution of the log-likelihood ratio. 


\subsubsection{Numerical density estimation using measured $\mathrm{S}_{\mathrm{V}}$ and modeled TS}

\section{Narrowband semi-empirical TS model}

The averaged $\mathrm{S}_{\mathrm{V}}$ centered at $38 \mathrm{kHz}$ with a bandwidth $3.4 \mathrm{kHz}$ is compared with TS predictions given by the narrowband, depth-dependent semi-empirical model proposed by Ona (2003) (hereafter referred to as the "narrowband TS model") to estimate the numerical density of fish. This method is chosen in order to imitate the procedure involved in conventional fisheries acoustic studies where narrowband signals and empirical or semi-empirical models are used (Simmonds and MacLennan, 2006). This model was derived by regressional analysis of TS measurements from a series of in-situ experiments where the effect of depth-dependent swimbladder compression is considered. The model is reproduced here for reference:

$$
\mathrm{TS}_{\mathrm{NB}}=20 \log L_{f}-2.3 \log (1+z / 10)-65.4,
$$

where $\mathrm{TS}_{\mathrm{NB}}$ denotes target strength prediction, $L_{f}$ is the length of the fish in $\mathrm{cm}$, and $z$ is the depth of the fish in $\mathrm{m}$. The measured distribution of the length of herring (Fig. 4-9) and the mean depth of the analysis window were used in the model prediction. The numerical density of fish estimated using this narrowband TS model, $\rho_{\mathrm{NB}}$, is given by

$$
\rho_{\mathrm{NB}}=10^{\left(\mathrm{S}_{\mathrm{V}, \mathrm{NB}}-\mathrm{TS}_{\mathrm{NB}}\right) / 10},
$$

where $\mathrm{S}_{\mathrm{V}, \mathrm{NB}}$ is the measured volume backscattering strength averaged over the band between $36.3 \mathrm{kHz}$ and $39.7 \mathrm{kHz}$ [marked by the vertical shades in Fig. 4-11(b)].

\section{Broadband physics-based hybrid TS model}

The $\mathrm{S}_{\mathrm{V}}$ measured across the Shamu, 424, and AirMarLow channels is compared with TS predictions given by the broadband physics-based hybrid TS model proposed by Chu et al. (2006) (here after referred to as the "broadband TS model") to estimate the numerical density of fish. This broadband TS model involves coherent summa- 


\begin{tabular}{ccc}
\hline & $g$ & $h$ \\
\hline Swimbladder & $0.0012(1+0.1 D)^{\gamma_{0}}$ & $0.22(1+0.1 D)^{\left(1-\gamma_{0}\right) / 2}$ \\
Fish body & 1.05 & 1.05 \\
\hline
\end{tabular}

Table 4.1: Summary of model parameters used in the broadband physics-based hybrid model. Here, the ratio of specific heat, $\gamma_{0}$, is 1.4 , and $D$ is the depth of the fish in meters.

tion of the backscattering cross section predictions from the swimbladder and the fish body. The gas-filled prolate spheroid model proposed by Ye (1997) is used to predict the swimbladder resonance in the low frequency region (approximately $<10$ $\mathrm{kHz}$ ), and the modal-series-based deformed cylinder solution is used to describe the scattering by the swimbladder at higher frequencies (Stanton, 1989). The model for scattering from the fish swimbladder is produced by connecting the low-frequency and high-frequency backscattering cross section predictions at around $10 \mathrm{kHz}$ using a smoothing function. For all frequencies, the distorted-wave Born approximation (DWBA, Morse and Ingard, 1987; Stanton et al., 1993b) evaluated using a fluid prolate spheroid is used describe the scattering from the weakly-scattering fish flesh. The predicted backscattering cross sections for the swimbladder and the fish body are then coherently combined to give the final broadband physics-based hybrid models. The values of model parameters are summarized in Table 4.1.

Instead of the prolate spheroidal shape used in Stanton et al. (2010), the geometry of the swimbladder is modeled here using the cross-sectional profile of a prolate spheroid but with its centerline adjusted to achieve a flat dorsal surface [Fig. 4-14(a)]. This modified shape bears a better resemblance to the typical shape of a herring swimbladder, and with its more directional scattering pattern, was found to produce more biologically-reasonable inference results in terms of the distribution of angles of orientation of fish when compared to the results produced using the original prolate spheroid geometry.

A separate investigation was also conducted to compare the predictions of swimbladder resonance given by Love (1978) and Ye (1997). Specifically, the models are implemented here using eq.(63) in Love (1978) and eq.(33) in Ye (1997). These two models differ primarily in the shapes used to present the fish swimbladder. Love's 

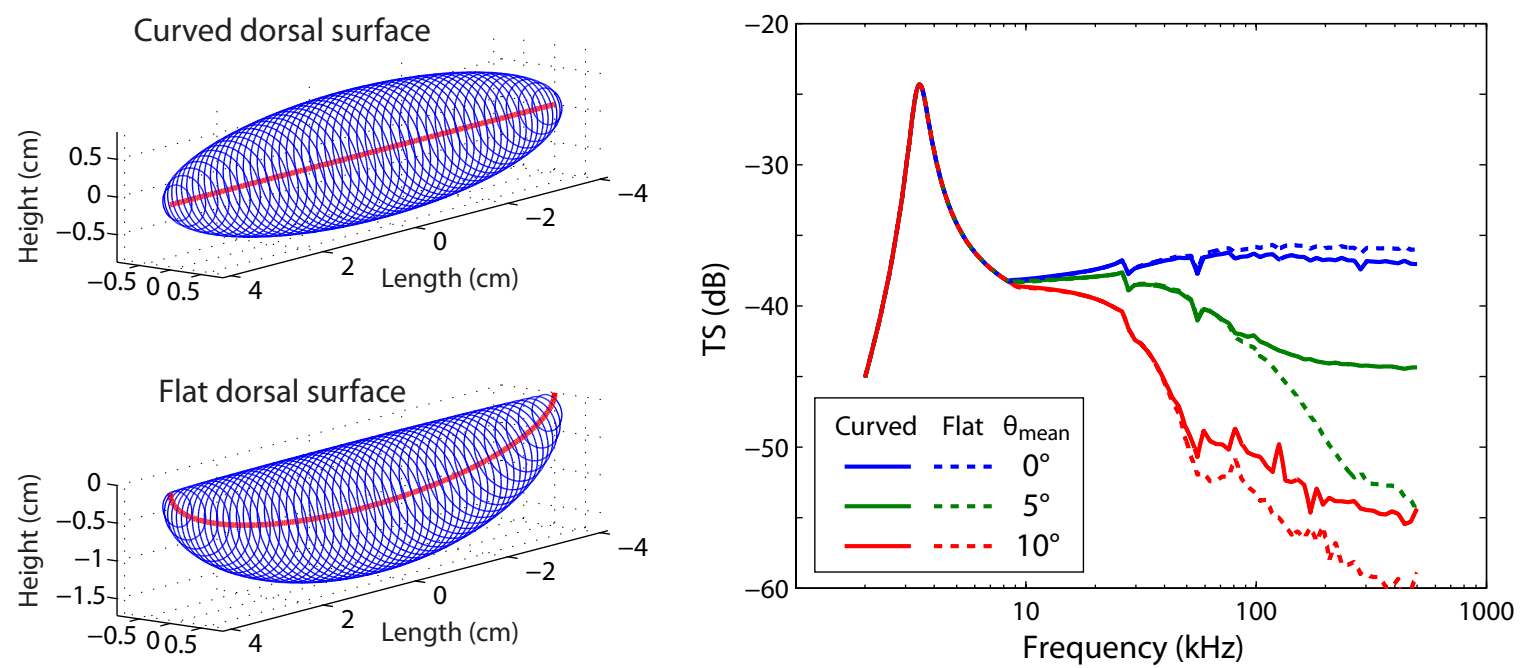

Figure 4-14: (a) Two model shapes for the fish swimbladder. The first model uses the shape of a prolate spheroid. The second model uses the cross-sectional profile of a prolate spheroid, but with its center adjusted to achieve a flat dorsal surface. The specific shapes shown here are generated assuming a swimbladder volume of $13 \mathrm{~mL}$ at sea surface for a $25 \mathrm{~cm}$ herring. (b) Examples of predicted averaged TS of this fish at a depth of $170 \mathrm{~m}$ evaluated using different angle of orientation distributions. The distributions of angle of orientation used here are normal distributions with different mean values $\left(\theta_{\text {mean }}=0^{\circ}, 5^{\circ}\right.$, and $\left.10^{\circ}\right)$ and an identical standard deviation $\left(\theta_{\text {std }}=3^{\circ}\right)$.

model was derived using modal-series expansion on gas-filled spherical shells with equivalent volume to the swimbladder with the resonance frequency heuristically corrected using the relationship given by Weston (1967). Ye's model was derived by evaluating the Kirchhoff integral over a volume-equivalent prolate spheroid. Ye also showed that his formulation can be reduced to exactly the results in Love (1978) by setting the aspect ratio to 1 in eq.(30-31) and eq.(35-36) in Ye (1997). However, it is observed that the TS predicted by these two models differ by approximately $6 \mathrm{~dB}$ in the low frequency region investigated (Fig. 4-15). This discrepancy is likely resulted from the extra factors accounting for the aspect ratio of the spheroid in Ye's model, which are completely absent in Love's model due to its assumption of a spherical shell. Nevertheless, the exact cause of this discrepancy is out of the scope of this study and further research is needed to resolve this issue.

In this study, Ye's model was selected for modeling the swimbladder resonance due to its better consistency with TS predictions at the transition $(\approx 10 \mathrm{kHz})$ between 


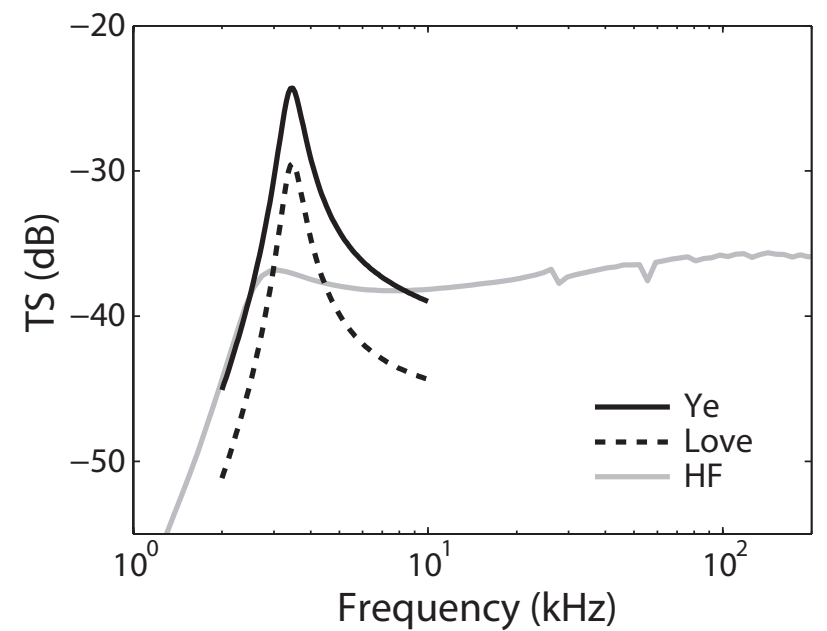

Figure 4-15: Comparison of TS predictions of swimbladder resonance given by two models. The first model (Ye) was derived assuming a prolate spheroidal shape (Ye, 1997), whereas the second model (Love) assumes a spherical shape with equivalent volume (Love, 1978). The low-frequency TS predictions are plotted along with the highfrequency TS predictions given by the modal-series-based deformed cylinder model (HF) using a swimbladder shape with flat dorsal surface. The calculation uses the same parameters as in Fig. 4-14.

the low-frequency resonance and high-frequency predictions given by the deformed cylinder model (Fig. 4-15).

A set of broadband TS models is constructed using a set of parameters including a range of fish flesh viscosities ( $\xi$, Fig. 4-16) and a distribution of angles of orientation of fish described by a normal distribution with mean $\theta_{\text {mean }}$ and standard deviation $\theta_{\text {std }}$ (Fig. 4-14). A standard swimbladder volume for a $25 \mathrm{~cm}$ fish at sea surface $\left(V_{0}\right)$ is back-calculated from the observed resonance frequency at the depth of the analysis window by evoking Boyle's Law and the neutral buoyancy assumption (Nero et al., 2004). Recall that $25 \mathrm{~cm}$ is the mean length of the herring sampled by the trawl (Fig. 4-9). The resonance frequency is determined by fitting the observed $\mathrm{S}_{\mathrm{V}}$ in the Shamu channel using a third-order polynomial and interpolate to obtain the location of the spectral peak. The swimbladder volumes for different sizes of fish are then calculated by scaling $V_{0}$ isometrically according to the length of fish. The values of $\theta_{\text {mean }}$ are constrained by the tilt of the swimbladder in the fish body $\left(\approx 5^{\circ}\right.$ relative to the body axis of herring, and the values of $\theta_{\text {std }}$ are varied arbitrarily within a 


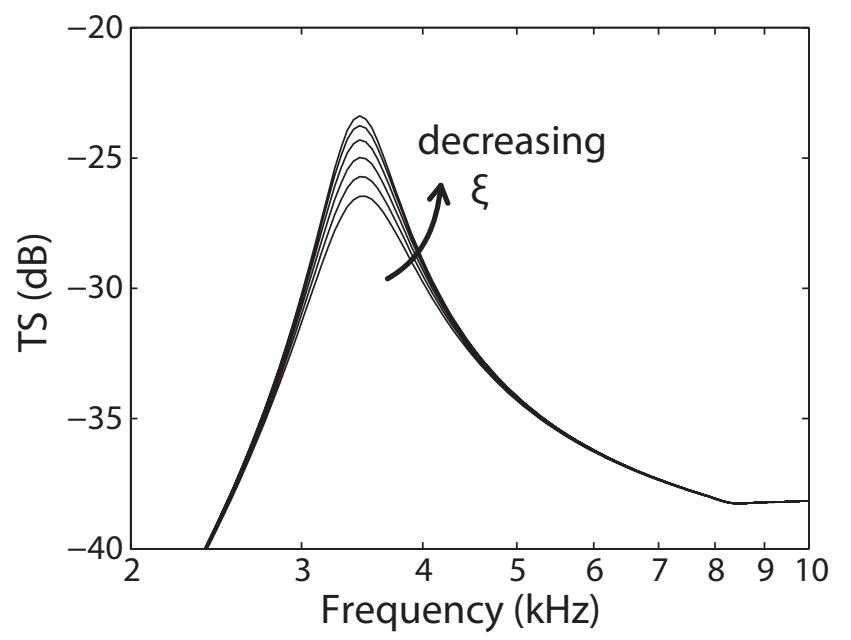

Figure 4-16: Comparison of TS predictions in the swimbladder resonance region with different values of viscosity for fish flesh $(\xi)$ using Ye's model (Ye, 1997). Here, the value of $\xi$ varies from 10 to $60 \mathrm{~Pa} \cdot \mathrm{s}$ at an interval of $10 \mathrm{~Pa} \cdot \mathrm{s}$. Calculations were made using the same parameters as in Fig. 4-14 and Fig. 4-15.

biologically-reasonable range between $1^{\circ}$ to $20^{\circ}$.

The best-fitting broadband TS model, $\mathrm{TS}_{\mathrm{BB}}$, was determined by a least-squares fitting procedure in the logarithmic scale to the shape of the observed volume backscattering spectra. The numerical density of fish estimated using this broadband physicsbased hybrid models, $\rho_{\mathrm{BB}}$, is given by

$$
\rho_{\mathrm{BB}}=10^{\left(\mathrm{S}_{\mathrm{V}, \mathrm{BB}}-\mathrm{TS}_{\mathrm{BB}}\right) / 10}
$$

where $\mathrm{S}_{\mathrm{V}, \mathrm{BB}}$ is the measured volume backscattering strengths across all three broadband channels (Fig. 4-11).

The confidence intervals for both the narrowband and broadband TS models are calculated indirectly through the confidence intervals associated with the spectral estimation for $\mathrm{S}_{\mathrm{V}}$. In other words, the upper and lower bounds for the density estimation are obtained by fitting the TS models to the upper and lower bounds of the $\mathrm{S}_{\mathrm{V}}$ estimation. In this study, the spectral level of $\mathrm{S}_{\mathrm{V}}$ and associated confidence intervals are calculated using the Welch method (Welch, 1967). 


\subsubsection{Comparison of the estimated numerical density of fish}

The numerical density of fish estimated using the broadband echo pdf model is compared to the density estimated using the two TS models described in Sec. 4.4.2 (Fig. 417). These estimations are fundamentally different in the inference approaches, since the inference given by the broadband echo pdf model is made based on the fluctuations of echoes, whereas the inference given by the TS models is based on the mean echo energy obtained through echo integration. This comparison gives an indirect assessment of the performance of the broadband echo pdf as an inference tool for the analysis of echoes collected in the field. The assessment is "indirect" due to the lack of groundtruth information on the actual numerical density of fish in the analyzed aggregation, even though the use of narrowband TS model in combination with echo integration is the industry standard in fisheries acoustics studies and the results are generally accepted as credible (Simmonds and MacLennan, 2006). Direct assessment of the performance of the broadband echo pdf model may be possible and is discussed as a future research direction in Sec. 5.2.2.

The numerical densities of fish estimated using the broadband echo pdf model by KL divergence and MLE are generally comparable for all analysis windows investigated (Fig. 4-17, red circles and black crosses). These statistics-based estimations are also comparable to the numerical densities estimated using the narrowband TS model (Fig. 4-17, blue squares), but are lower than and completely outside of the confidence intervals of the estimations given by the broadband TS model (Fig. 4-17, green triangles). However, the general trend of the variation of the estimated numerical density of fish within the aggregation across multiple analysis windows is similar for all three models, with higher numerical density toward the center and lower numerical density toward the edges of the fish aggregation (Fig. 4-17). The agreement in the trend of the estimated numerical density establishes the first-order applicability of echo statistics methods on data collected in the field. Potential sources of errors associated with each method are discussed in Sec. 4.4.4. 
(a)

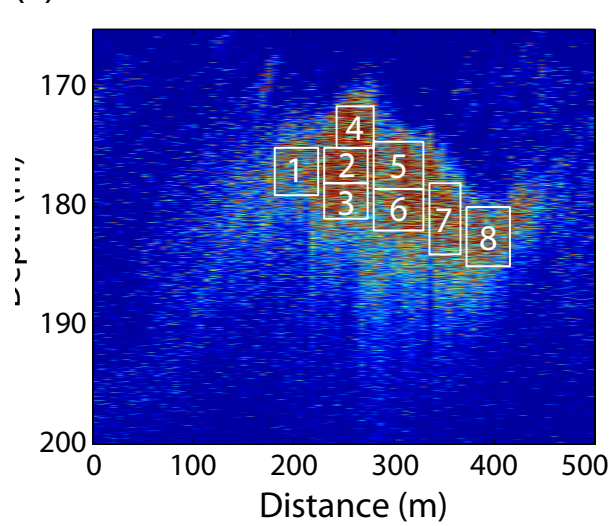

(b)

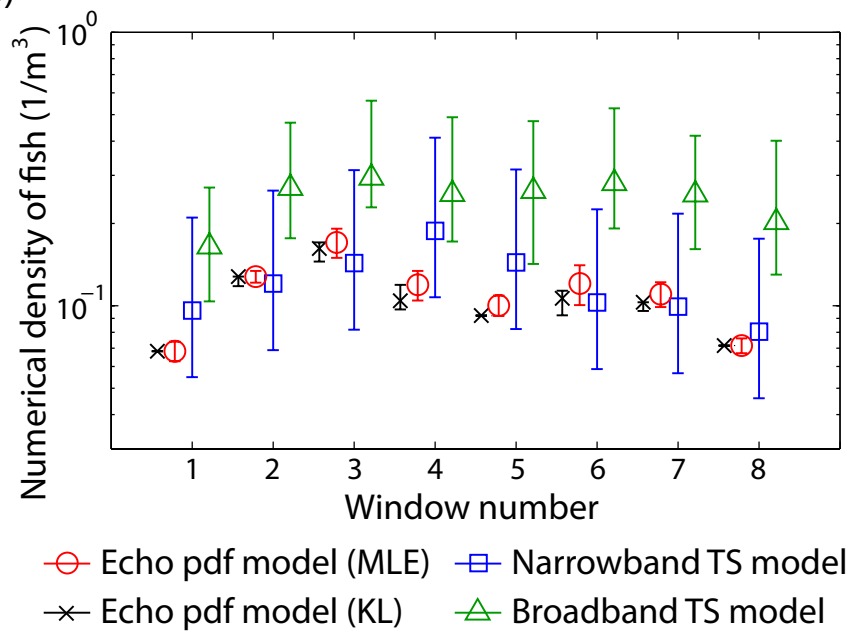

Figure 4-17: (a) Echogram of the monospecific herring aggregation with analysis windows. Windows \#1 and \#2 are identical to the two windows shown in Fig. 4-11. (b) Numerical density of fish estimated using various methods. Results are plotted along with their respective confidence intervals.

\subsubsection{Errors associated with the estimation of the numerical density of fish}

Errors in the estimation given by narrowband and broadband TS models

The errors associated with the numerical density estimations given by the narrowband and broadband TS models are subject to different constraints imposed by the assumptions and parameterization used in each model. For example, the narrowband TS model was derived using measurements from Atlantic herring in Norwegian waters (Ona, 2003). Therefore, the application of this model on data collected in the Gulf of Maine represents an extrapolation to this regression model (Fässler et al., 2008) and is likely to produce biased results. This is because the applicability of empirical or semi-empirical TS models are generally justified by the assumption that the distribution of angle of orientation, behavior, and life history state of the measured population are representative of the target population (Sec. 1.3.2). Extrapolation of the models without detailed verification of these parameters using groundtruth information results in violation to this assumption and is expected to cause error.

However, the relatively simple formulation of the narrowband TS model avoids 
the challenges posed by the large number of nuisance parameters in the broadband TS model. Nuisance parameters are parameters that are not of immediate interest but must be accounted for to infer the parameters of interest. For the broadband TS model discussed here, nuisance parameters include the distribution of the angles of orientation and lengths of fish, the viscosity of fish flesh, the shape of fish swimbladder, as well as the volume and associated depth-related compression of the swimbladder. Although groundtruth information from trawl catches and prior knowledge about the survey region were used to constrain these parameters, and a best-fit procedure (Sec. 4.4.2) was used to optimize the selection of parameter values, the sensitivity of model output with respect to uncertainties in each parameter has to be quantified to give a reliable measure of the accuracy of this model (see Sec. 5.2.3 for future discussion).

\section{Errors in the estimation given by broadband echo pdf models}

Errors associated with the estimated numerical density of fish using broadband echo pdf models are directly related to parameters selected in the model implementation. This section discusses several important potential sources of errors and their corresponding effects on the estimation results (Table 4.2).

First of all, the amplitudes of echoes from each individual scatterer are assumed to be Rayleigh-distributed in the model implementation. However, the validity of this assumption depends on the behavioral state and the distribution of angle of orientation of the fish within the aggregation. For example, it has been shown through data collected in laboratory experiments that the echo amplitudes from individual live fish can contain strong non-Rayleigh features when the fish was calm or physically constrained in a harness, such that the echoes are primarily from the scattering contributions of only few dominate sources, such as the swimbladder, across a narrow range of angles of orientation (Clay and Heist, 1984; Stanton et al., 2004). The same sets of experiments also showed that when the fish was active or when echoes from all angles of orientations are pooled, the scattering from other body parts of the fish dominates the echoes, and the resultant echo pdfs can be very Rayleigh-like, 


\begin{tabular}{lc}
\hline \multicolumn{1}{c}{ Model assumption } & $\begin{array}{c}\text { Influence on estimation result if the } \\
\text { model assumption is violated }\end{array}$ \\
\hline $\begin{array}{l}\text { Echoes from individual fish are Rayleigh- } \\
\text { distributed }\end{array}$ & Underestimate \\
$\begin{array}{l}\text { All fish are of the same size in the selected } \\
\text { fish aggregation }\end{array}$ & Underestimate \\
$\begin{array}{l}\text { The fish are randomly distributed in the } \\
\text { entire hemispherical space enclosed by the } \\
\text { analysis window }\end{array}$ & Underestimate \\
$\begin{array}{l}\text { The fish has uniform frequency response } \\
\text { across the band of interest }\end{array}$ & Overestimate \\
\hline
\end{tabular}

Table 4.2: Summary of potential sources of errors and corresponding impact on the estimation results associated with model assumptions used in this study.

especially for echoes collected at higher frequencies.

In this study, since the echo data were collected from dense monospecific herring aggregations using a downward-looking sonar beam, it is highly likely that the fish were insonified across a limited range of angles of orientation, and that the resultant echo pdf for individual fish would be strongly non-Rayleigh. This argument is strengthened by the result that the standard deviation of angle of orientation $\left(\theta_{\text {std }}\right)$ associated with the best-fitting broadband TS model generally falls between $10^{\circ}$ to $17^{\circ}$ for the analysis windows examined. According to the results of the comparison of broadband echo pdf models generated assuming Rayleigh-distributed scatterers and randomly-rough prolate spheroids (Fig. 4-7), it is expected that the incorporation of non-Rayleigh-distributed individual scatterers will lead to broadband echo pdf models with stronger non-Rayleigh features. This would cause the current model to underestimate the numerical density of fish in the observed aggregation.

The current model also assumes that the mean backscattering amplitude is identical for all scatterers, which is not realistic considering the distribution of fish length revealed by trawl catches (Fig. 4-9). Since the mean echo amplitude varies according to the size of the scatterer, echo pdfs produced by scatterers of a range of sizes 
will tend more toward the Rayleigh distribution compared to echo pdfs produced by the same number of identical scatterers. Therefore, the current model is likely to underestimate the numerical density of fish.

On the system level, the current model assumes that the scatterers are randomlydistributed in the entire space enclosed in the hemispherical shell between the range gate (Fig. 4-1). However, the fish aggregations observed in the experiment had a finite vertical extent under the towbody (i.e., a layer). Therefore, echoes are more likely to originate from scatterers located in or near the mainlobe of the sonar beam, which would give rise to a heavier tail in the echo pdf when the number of scatterers within the gate is the same. In this case, the current model would underestimate the numerical density of fish in the observed aggregation.

It is also known that for elongated scatterers such as fish, the directionality of the scattering function increases with increasing frequencies (Medwin and Clay, 1998). Therefore, for fish schools with a particular distribution of angle of orientation, the measured volume backscattering strength is likely to decrease with increasing frequency (Fig. 4-14). These characteristics will create an effect similar to a low-pass filter on the signals and also reduces the signal bandwidth. Based on the results of Sec. 4.2.2, these modifications will cause the echo pdf to tend toward the Rayleigh distribution. Therefore, the current model evaluated assuming uniform frequency response for all scatterers is likely to overestimate the numerical density of fish. This is to the contrast of the influence of the other factors discussed above.

The accuracy of the numerical density of fish estimated by the broadband echo pdf model depends on the specific choices of parameters in model implementation and the actual experimental scenario in the field, and detailed sensitivity analysis on the scale of influence and the relative importance of each of the factors is clearly needed (see Sec. 5.2.2 and Sec. 5.2.3). However, given the fact that the distribution of angle of orientation of fish is unknown and that the other three factors can easily be violated in the current experimental scenario, it is more likely that the numerical densities of fish given by the broadband echo pdf model reported here are underestimates. The results can thus also be interpreted as effectively provide a set of lower bounds for 
the numerical density of fish in the observed aggregation.

\subsection{Summary and Conclusion}

A Monte Carlo numerical simulation framework has been developed to model the probability density function of the magnitudes of complex envelopes of pulse-compressed echoes (echo pdf) collected using a single-beam broadband echosounder. This model adopts a physics-based approach and rigorously accounts for the influences of sonar system parameters and signal and scatterer characteristics in the backscattering process. Broadband echo pdf model predicted using this new model were applied as an inference tool to estimate the numerical density of fish in monospecific herring aggregations observed in the ocean. The numerical density of fish estimated using the broadband echo pdf models are comparable but generally lower than the densities estimated using the other two methods involving $\mathrm{S}_{\mathrm{V}}$ measurements and modeled TS. Errors in the estimation can be explained by potential deviation of model parameters from the actual values.

The numerical model is constructed in the time domain based on the temporallylocalized features of broadband pulse-compressed echoes. The modeling framework allows the incorporation of various frequency-dependent components, including the beampattern effects (Chu and Stanton, 2010; Ehrenberg, 1972), response of the sonar system (from both the transducer and system electronics), features of the transmit signal, scatterer characteristics including the distribution of size and angle of orientation, and spatial distribution of these scatterers in the sampling volume (Fig. 4-1 and Fig. 4-2). Using the exact parameters of the AirMarLow transducer in the EdgeTech broadband echosounder system, the model is implemented and applied to predict the echo pdfs from arbitrary number of scatterers observed through an axisymmetrical circular aperture using linear chirp signals $(30-70 \mathrm{kHz})$.

The shapes of broadband echo pdfs are sensitive to the frequency content and bandwidths of the echoes, which are jointly determined by characteristics of the system, signals, and scatterers. Signals with broader bandwidths or more high frequency 
energy tend to produce echoes with strong non-Rayleigh characteristics, whereas signals that are narrowband or contain more low frequency energy are more likely to produce echo pdfs with more Rayleigh-like features. Similar to the cases where narrowband signals are considered (Chu and Stanton, 2010), the shape of the broadband echo pdf approaches the Rayleigh distribution with increasing number of scatterers in the specified sampling volume (Fig. 4-1 and Fig. 4-13), and the presence of more than one size class of scatterer in mixed assemblages will lead to dramatic changes in the shape of the resultant echo pdf (Fig. 4-8, also see Chapter 3).

Monospecific herring aggregations observed near the seafloor during day time are selected for analysis with an aim to assess the performance of the broadband echo pdf model as an inference tool for estimating the numerical density of fish in the aggregation. The numerical density of fish is estimated using two methods. The first method involves curve-fitting by minimizing the KL divergence between the echo pdf of the data and the model, and the second method uses a maximum likelihood estimator. Results of the estimated numerical density of fish are compared to the density estimated using echo integration techniques in combination with a narrowband semiempirical TS model (Ona, 2003) and a broadband physics-based hybrid model (Chu et al., 2006) .

The numerical densities of fish estimated independently from the above three models are comparable, but the estimation given by the broadband echo pdf model is generally lower than the densities estimated by the other two models. The discrepancy can be explained by potential mismatches between model assumptions and the actual values of corresponding parameters in the backscattering process. The results suggest that better groundtruth information and more detailed understanding of the echo statistics properties from individual fish can improve the performance and applicability of the broadband echo pdf model.

The broadband echo pdf model is constructed based on physics principles of the transmission, scattering, and reception of sound, and thereby provides explicit links between features of the echo pdf and the sources of scattering. Due to the same reason, although this model is inspired and developed in the context of acoustic scattering 
study of marine organisms and the analysis presented here focuses on the echoes collected by a downward-looking single-beam broadband echosounder in a direct-path, volume-backscattering geometry, this model is generally applicable to other types of scattering sources in different environments observed using different systems. For example, the proposed numerical modeling framework can be implemented to predict and analyze the scattering of electromagnetic signals from aerial objects observed by radar, as well as fish echoes in a multi-path environment, such as an ocean waveguide (Jones, 2012). Results of this study suggest that the statistics of echo fluctuations can be used as an additional dimension of information in complement to the spectral and temporal features for accurate interpretation of echo data. 


\section{Chapter 5}

\section{Summary of contributions and recommendations for future research directions}

The research in this thesis focuses on the analysis of echoes from random scatterers, inspired by the need for accurate and reliable acoustic scattering techniques for echo interpretation in the context of the study of marine organisms. This research makes contributions to the field of active acoustic sensing of the ocean by providing new data and models in combination with a unique approach that can be summarized by: 1) quantitative characterization of the spectral, temporal, and statistical features derived from echoes collected using both broadband and narrowband signals, and 2) a physicsbased modeling approach that aims to interpret the echoes by connecting echo features to the physics of acoustic scattering and the influence of the sensing systems. These are distinct from the approaches taken by conventional fisheries acoustic studies which generally make heavy reference to subjective morphological description of biological aggregations observed on the echogram, rely primarily on the echo levels at one or several narrowband frequencies, and analyze echoes using empirical- or semi-empirical data-driven models (Sec. 1.3). A summary of the contributions and significance of this research is given in the sections below, followed by recommendations for future research directions and a discussion of the broader impact of this study on active 
remote-sensing using sonar and radar, and in particular, its implications for animal echolocation studies.

\subsection{Contributions and significance}

\subsubsection{Broadband backscattering from live squid}

Chapter 2 investigates the acoustic backscattering from live squid through a combination of laboratory measurements and modeling. Building on the understanding of basic principles that governs the scattering from squid in the literature (Au and Benoit-Bird, 2008; Benoit-Bird et al., 2008; Kang et al., 2005; Madsen et al., 2007; Starr et al., 1998), this study is unique in providing the first broadband laboratory backscattering data set for squid at all azimuthal angles of orientation and novel in the analysis of echoes that involves a series of detailed data-model comparisons in the angular-dependent spectral and temporal features for both laboratory and simulated field conditions. The results conclusively confirm the backscattering mechanisms of squid across all angles of orientation and confirm the fluidlike, weakly-scattering properties of squid. This study also successfully demonstrates that the three-dimensional numerical distorted-wave Born approximation (DWBA) model is capable of predicting squid TS over a wide range of angles of orientation provided that proper shape representation is derived using groundtruth information such as optical images or direct observation of the squid behavior. These findings serve as the basis for the parameterization of squid scattering models that is critical for accurate interpretation of squid echoes collected in field. This study also reveals that echoes from individual randomly-oriented squid contain strong non-Rayleigh characteristics, which can be further exploited for the purposes of scatterer discrimination, classification, or identification.

The results of this study are crucial in providing better estimation of the abundance and distribution of squid in the ocean by using active acoustic survey methods. Despite the importance of squid in global commercial fisheries and their key roles 
in the marine ecosystems as both predator and prey, such information has remained crude due to the lack of appropriate survey techniques that can counteract the effective net-avoidance capabilities of these rapidly-moving animals. The modeling tools verified in this study also provide a means of predicting the scattering from many squid species that are not otherwise available for controlled laboratory measurements owing to the difficulties associated with specimen handling. This is particularly important in the studies of midwater fauna, of which squid are a major component (Boyle and Rodhouse, 2005), since human exploration of deep waters have increased dramatically in recent years as a result of depletion of shallower water resources (Pauly et al., 2002; Roberts, 2002). Combined with the information from other empirical backscattering measurements of squid (Au and Benoit-Bird, 2008; Benoit-Bird et al., 2008; Kang et al., 2005; Madsen et al., 2007; Starr et al., 1998), results of this study can further be applied to aid the interpretation of foraging behaviors of many deep-diving toothed whales, who feed predominantly on midwater squid through echolocation.

\subsubsection{Statistics of echoes from arbitrary aggregations of scat- terers}

Chapter 3 and Chapter 4 are devoted to the development and application of statistical models for the probability distribution of echo amplitudes (echo pdf) from arbitrary aggregations of scatterers. In contrast to the laboratory studies designated to elucidate the backscattering mechanisms of individual squid, the echo statistics models are developed based on typical scenarios in field experiments where parameters related to the composition of the scatterer aggregations are often the goal of echo interpretation. Examples of such parameters for biological aggregations include the type of organisms as well as their numerical densities and distribution within the aggregation. Here, different types of oragnisms are characterized by their physical scattering properties, which are determined by the acoustic frequency and the acoustic boundary conditions, sizes, and shapes of the organisms. The scatterers are generally not resolved, and the geometry is in the backscattering direction in the direct-path case 
where there are no interfering echoes from neighboring boundaries. The study here is aimed at establishing the statistics of echoes as a new dimension of information that is complementary to the commonly-used spectral and temporal echo features, with the ultimate goal of accurate interpretation of echoes for biologically-relevant information. An example for the joint use of spectral and echo statistics information is demonstrated in Chapter 4 in the analysis of broadband echoes collected in the ocean.

\section{Statistics of echoes from arbitrary aggregations of scatterers measured using narrowband signals}

In Chapter 3, a theoretical continuous-wave (CW) model is developed for describing the echo pdf from arbitrary aggregations. This model is an extension of the formulation presented in Chu and Stanton (2010) and is specifically applied in this study for predicting and analyzing the echoes from mixed assemblages of scatterers. Mixed assemblages are defined here for cases in which more than one type of scatterer are spatially-interspersed and uniformly-distributed within the volume enclosed by the analysis window. Such scenario is prevalent in important marine ecological processes, such as foraging, where animals from multiple trophic levels are spatially collocated (Benoit-Bird, 2009a; De Robertis, 2002). From a broader perspective, mixed assemblages are also ubiquitous in a wide range of terrestrial and oceanographic contexts that can be measured using either acoustic or electromagnetic signals. Examples include mixed vegetation composition across landscapes observed using radar (GreigSmith, 1984), and sonar observation of sub-sea surface bubble plumes (Woolf, 1997) and geophysical features on the seafloor (Heezen and Hollister, 1971).

In contrast to the conventional data-driven approach which focuses primarily on describing the statistics of mixed assemblage echoes using mixture models (weighted sum of pdfs) (e.g., Abraham and Lyons, 2010; Watts and Wards, 2010), the physicsbased model developed here directly connects the statistical features in the echoes with the composition of mixed assemblages and the influence of the transducer beampattern of the sensing system. When used as an inference tool for analyzing simulated 
mixed assemblages, this new model achieves significant improvement in the accuracy of the inferred parameters of assemblage composition, sometimes by an order of magnitude, when the inference results are compared with those of the mixture models. Through the development and application of this model, this study demonstrates the superior performance of physics-based models over data-driven models in the interpretation of echoes and shows that echo statistics methods can be useful in the analysis of mixed assemblages of scatterers.

\section{Statistics of echoes from arbitrary aggregations of scatterers measured using broadband signals}

Although the study in Chapter 3 demonstrated the power of physics-based echo statistics models in echo interpretation, the narrowband $(\mathrm{CW})$ model may be of limited use in the analysis of data collected in the field, since the discriminative power of echo statistics models depends on the presence of strong non-Rayleigh echo features that may not be regularly encountered in typical in-situ experimental scenarios. This is due to the limited temporal, and thus spatial, resolution of narrowband signals coupled with increasing sampling volumes at depth, which result in significant overlap of echoes and consequently Rayleigh-like statistical characteristics. This problem motivates the development of a model that is capable of predicting the statistics of the magnitudes of broadband pulse-compressed echo envelopes from arbitrary aggregations in Chapter 4. This model takes advantage of the substantially-improved temporal resolution of broadband signals after pulse compression processing, which gives stronger non-Rayleigh echo features compared to narrowband signals. The model development is also motivated by the need for a tool capable of providing statistical information from broadband echoes to complement the rich temporal and spectral information obtained using broadband signals in the field (Sec. 1.4).

The new broadband echo pdf model is developed under a Monte-Carlo numerical modeling framework, and is implemented using the exact system parameters and transducer beampattern of a broadband echosounder $(30-70 \mathrm{kHz})$ deployed in field experiments. The models are used to estimate the numerical density of fish in 
monospecific herring aggregations over Georges Bank in the Gulf of Maine, identified using the resonance peak on the spectrum. This particular one-parameter estimation scenario is selected in order to simplify the inference procedure and also in view of the importance of the numerical density of organisms in the study of biology in the ocean. The numerical density of fish estimated using the broadband echo pdf model is generally comparable to the densities estimated using two conventional methods, which involve comparing measured volume backscattering strengths to target strengths predicted using a broadband physics-based spectral model and a semi-empirical regression model. This result shows that echo statistics methods, which can be applied without detailed calibration of the sensing system, can infer biologically-relevant information from the echoes and thereby establishes the firstorder applicability of broadband echo pdf models in the interpretation of echo data collected in the field.

\subsubsection{Summary of contributions of thesis work}

The contributions achieved in this thesis are itemized below:

- Collected the first broadband laboratory backscattering data set from individual live squid at all angles of orientation (Chapter 2)

- Confirmed the fluidlike, weakly-scattering properties of squid through a series of detailed data-model comparisons in the spectral and temporal domain over all angles of orientation (Chapter 2)

- Improved the parameterization of the three-dimensional distorted-wave Born approximation numerical model for predicting the scattering from squid by investigating the influence of different shape representations (Chapter 2)

- Revealed the strong non-Rayleigh statistics features in the echoes from randomlyoriented individual squid (Chapter 2)

- Developed a physics-based analytical narrowband (continuous-wave) model for predicting the probability distribution of echo amplitudes (narrowband echo 
pdf) from arbitrary aggregations of random scatterers (Chapter 3)

- Demonstrated that the physics-based narrowband echo pdf model can achieve order-of-magnitude performance improvement compared to conventional datadriven mixture models when used as an inference tool to analyze the composition of mixed assemblages (Chapter 3)

- Developed a physics-based numerical model for predicting the probability distribution of the magnitudes of broadband pulse-compressed echo envelopes (broadband echo pdf) from arbitrary aggregations of random scatterers (Chapter 4)

- Applied the physics-based broadband echo pdf model to broadband fish echoes $(30-70 \mathrm{kHz})$ collected in the ocean, which produced estimations of the numerical density of fish that are comparable to the densities estimated using conventional acoustic-based methods that involve volume backscattering strength measurements and target strength models (Chapter 4)

- Established the first-order applicability of the physics-based broadband echo pdf model in the interpretation of echo data collected in the field (Chapter 4)

\subsection{Recommendations for future research direc- tions}

\subsubsection{Backscattering from squid}

Concurrent in-situ acoustic and optical observation of different squid species

Results of Chapter 2 showed that the success of the three-dimensional numerical DWBA model in predicting the target strength of squid depends considerably on the choice of the shape representation of squid. Therefore, in-situ groundtruth information via net- or optics-based methods capable of delivering information on the general shape of the target squid species is crucial for the application of this model for analyzing data collected in the field. A potentially fruitful experimental approach 
would involve simultaneous deployment of collocated broadband echosounder and video or still-image cameras on remote operated vehicles (ROV), with a goal to collect concurrent optical and acoustic backscattering data from different squid species distributed in different layers of the water column. Combined with the application of the three-dimensional DWBA numerical model, this type of direct measurements with groundtruth information will significantly increase the reliability of subsequent analysis of acoustic data collected over a larger volume at similar spatial location and depth.

\section{Measurements of material properties of a wide range of squid species}

The concurrent acoustic and optical observation of squid proposed above would also allow further verification of the applicability of the DWBA model in predicting the scattering from this diverse group of animals that are known to possess distinct muscular composition depending on the physiological demand of each species according to their ecological niche (O'Dor, 2002; Seibel et al., 2004). Specifically, more extensive measurement of the material properties (mass density, $g$, and sound speed, $h$ ) of the muscles of different squid species are needed to increase the accuracy of the DWBA model prediction (Smith et al., 2010). Important groups of squids to be investigated include the midwater ammoniacal squids (the "blimps", O'Dor, 2002), which are likely to have $g$ and $h$ values closer to 1 than the Loliginids studied here, and the more muscular species such as the Humbolt squid, which may require further investigation of more sophisticated scattering models due to the potential local material property changes from cartilage to bony structures for organs such as the skull (Benoit-Bird et al., 2008).

\section{Investigation of other potentially important scattering sources within squid}

As discussed in Sec. 2.6.3, the scattering contributions from squid body parts other than the fluidlike muscles remain an important topic of future research. For example, the potential scattering contribution from the gladius should be properly assessed using backscattering data collected in the dorsal-ventral plane as opposed to data 
in the lateral plane, as collected in Chapter 2. Potential species-specific structural changes, such as the one described above for Humboldt squid (D. gigas), should also be addressed. Transducers with broader bandwidths in combination with broader bandwidth signals that can achieve better temporal resolution should also be used in order to separate the potential scattering contribution from the beak or the skull from that of the arms of the squid.

\section{Development of echo statistics models for individual squid or other marine organisms}

Results in Sec. 2.5 showed that the statistics of echoes from individual squid at all angles of orientations can be described by the echo pdf from a randomly-rough, randomly oriented prolate spheroid with a smaller aspect ratio compared to the actual aspect ratio of the measured animal. The aspect ratio is considered "effective" due to: 1) the prolate spheroid is assumed to be impenetrable, which is not consistent with the material properties of squid muscles, and 2) the model roughness is introduced heuristically by modulating the echo amplitudes by the Rayleigh distribution, whereas a more realistic equivalent roughness of squid should be estimated through the correlation length of the temporal scattering features of squid. A new model capable of incorporating the weakly-scattering, and thus penetrable, material properties of squid and arbitrary roughness are expected to better describe the echo pdf from squid with more accurate estimation of the aspect ratio. Similar models can also be developed to re-examine the non-Rayleigh scattering properties observed from a variety of individual marine organisms, including zooplankton and fish (Stanton et al., 2004). Such echo statistics models for individual organisms provide a means to summarize the fluctuations in the echoes and may be useful for the discrimination of scattering sources and serve as building blocks for modeling the statistics of echoes from aggregations of scatterers. 


\subsubsection{Statistics of echoes from aggregations of scatterers}

\section{Application of the narrowband and broadband echo pdf models to mixed assemblages of marine organisms observed in the field}

The study in Chapter 3 and Chapter 4 showed: 1) the newly-developed narrowband mixed assemblage echo pdf model can be successfully applied as an inference tool to analyze the composition of simulated mixed assemblages, and 2) the broadband echo pdf model can be applied to estimate the numerical density of fish in monospecific aggregation of fish observed in the field. Based on these results, it is expected that the narrowband and broadband echo pdf models developed here can be used to analyze the composition of mixed assemblages of fish and/or zooplankton observed in the field. This type of data may be available from the four-frequency $(38,70$, 120 , and $200 \mathrm{kHz}$ ) narrowband echosounder systems that are commonly deployed for fisheries applications in recent years (Simmonds and MacLennan, 2006). However, the increasing number of free parameters associated with increasing complexity of mixed assemblages will result in an exponentially-growing number of possible echo pdf curves from all combination of parameters, which will render the best-fit procedures used in Chapter 3 and Chapter 4 highly inefficient. This problem is further exacerbated if the number of scatterer types are unknown in the mixed assemblage. Advanced sampling techniques, such as the Markov-Chain Monte-Carlo methods that are suitable for sampling multidimensional distributions, in combination with transdimensional Bayesian models which can handle variable dimensions, may be explored for a solution to these challenges (e.g., Chib et al., 2001; Quijano and Dosso, 2011).

Such analysis will allow further assessment of the applicability of echo pdf methods on field data and provide an opportunity for investigating the advantage of assimilating multidimensional (spectral, temporal, and statistical) features in echo interpretation. 


\section{Laboratory backscattering experiments for direct verification of echo pdf models}

The study in Chapter 4 provided a first-order assessment of the applicability of broadband echo pdf model in the analysis of data collected in the field by comparing the numerical density of fish estimated using the broadband echo statistics and two conventional TS-based methods. However, this performance evaluation was indirect since the actual numerical density of fish was unknown in the surveyed region. Direct verification of the performance of echo statistics methods may be achieved by applying the broadband echo pdf model to estimate the numerical density of scatterers using echoes collected in a laboratory backscattering experiment in which the number and scattering characteristics of either artificial or biological scatterers insonified by the sonar system is known. This data set from controlled experiment could also be used to investigate the sensitivity of the estimation results due to errors in model assumptions, such the frequency and amplitude response of individual scatterers and their spatial distribution, etc. (see Sec. 4.4.4 for detail).

\section{Analysis of the statistics of echoes across multiple narrowband or broad- band channels}

Since the scattering response of different sizes of scatterers is a strong function of frequency, comparison of the statistics of echoes across more than one narrowband or broadband channels may provide additional features that can be exploited for echo interpretation. This approach may be particularly valuable for analyzing the composition of mixed assemblages. For example, in a mixed assemblage composed of two size classes of fluidlike zooplankton, the statistics of echoes for lower frequency bands are more likely to be dominated by the larger zooplankton (effectively a monotype aggregation), while echoes from both sizes of zooplankton will contribute to the statistics of echoes in the higher frequency bands (effectively a mixed assemblage). The transition of echo pdf features, which is analogous to the spectral information in TS for different sizes of organisms, may be used for the discrimination and classifica- 
tion of scatterers. Such analysis may also be useful for the analysis of bubble plumes (Leighton, 1997), since the resonance structure of bubbles are likely to lead to high tails in the echo pdfs, and the transition of echo pdf features across multiple frequency bands may represent the transition of bubble densities along the size spectrum.

\section{Derivation of analytical broadband echo pdf model}

Another valuable research topic is the derivation of analytical solutions for the statistics of broadband echoes from arbitrary aggregations of scatterers. Such analytical models may be achievable by approximating the envelope of the compressed pulse output from individual scatterers using convenient general functions, such as the Gaussian function, approximating the oscillation using sinusoidal functions (analogous to a narrowband assumption of the envelope function), and constructing the echo pdf through Barakat's method of characteristic functions (Barakat, 1974). This type of model can be useful for providing important benchmark assessment of the sources of scattering before detailed echo statistics analysis is conducted using computationallyexpensive but rigorous numerical models. Furthermore, such a model, if capable of capturing the behavior of the tail of echo pdfs, will be invaluable in verifying and improving the numerical model, whose accuracy is inherently limited in the tail where the probability density function varies dramatically and is prone to bias in density estimation (Scott, 1992).

\section{Improvement of uncertainty analysis for the estimation of the numerical density of fish}

Although attempts were made in Chapter 4 to quantify the uncertainties of estimation results by reporting their associated confidence intervals, the treatment was not complete and can be improved. Specifically, in the cases where the numerical density of fish was estimated using modeled TS and volume backscattering $\left(\mathrm{S}_{\mathrm{V}}\right)$ measurements, only the uncertainties associated with the spectral estimation of $\mathrm{S}_{\mathrm{V}}$ were included. Uncertainties in the parameters of the TS models, such as the coefficients in regression models, should be incorporated by propagation of error (Bevington and 
Robinson, 2003). In the cases where the numerical density of fish was estimated using the broadband echo pdf model, uncertainties associated with many model parameters, a multidimensional pool of broadband echo pdf models can be constructed to include the uncertainties in model parameters, such as the statistical models for individual scatterers and their frequency-dependent scattering response, so that a confidence region involving multiple model parameters can be obtained (Azzalini, 1996). The inclusion of the uncertainties in these parameters are expected to broaden the confidence intervals but will provide a better assessment of the quality of the inference results.

\subsubsection{General approaches for the interpretation of echoes}

Based on the experience and insights gained from this thesis work, additional recommendations are made for future research directions related to general approaches for the interpretation of echoes and data collection.

\section{The influence of nuisance parameters in the context of acoustic scattering by marine organisms}

In the context of the study of acoustic scattering by marine organisms, the interpretation of echoes is usually limited by the large number of "nuisance parameters" that are important in determining the scattering from the organisms but are not the quantities of interest. For example, in cases where the goal of estimation is the numerical density of organisms in an aggregation, uncertainties associated with the distribution of the size and angle of orientation of the organisms, as well as variation in their shapes and material properties can significantly affect the estimation results. In conventional fisheries acoustic studies, these nuisance parameters are usually incorporated in model predictions using fixed values determined from prior experience and groundtruth information to simplify the analysis, and the influence of the uncertainties in these assumptions on the estimation results are generally ignored. Although sensitivity analysis of several important model parameters has been conducted for 
fish and zooplankton (Hazen and Horne, 2003; Lawson et al., 2006), there is a clear need for an overarching framework under which the influence of nuisance parameters can be investigated.

The Fisher information matrix and the associated Cramer-Rao bound provide a potential framework to address this issue. The Fisher information measures the amount of information available in the observation that can be used to infer unknown model parameters, with which the observation are associated (Kay, 1993). The Cramer-Rao bound is the inverse of the Fisher information and gives a lower bound to the variance of the estimator, which provides a measure of the quality of the estimator based on information in the observation. By decomposing the Fisher information matrix into elements determined by two exclusive sets of parameters consisting of desired parameters and nuisance parameters, respectively, it is possible to formally quantify the influence of these nuisance parameters on the estimation results (Scharf and McWhorter, 1993)

\section{Optimization of the model formulation for echo interpretation}

The analysis framework discussed above can also be used to theoretically or numerically quantify the improvement in the amount of information available for better inference as a result of different choices of model formulations, model parameters, and observations (echo features). Such analysis can lead to quantitative methods for optimizing the formulation of echo interpretation and selecting echo features specific to the scientific question and the goal of analysis. For example, the set of echo features chosen for the analysis of mixed assemblages of fish and squid and those chosen for the analysis of mixed assemblages of different sizes of zooplankton should intuitively be very different and may be revealed systematically through the above analysis.

This analysis can also aid in the design of new measurement protocols that can bring in additional information to improve the interpretation of echoes. One potential echo feature that can be explored is the strong angular dependency of the scattering functions of most marine organisms. In Chapter 2, the angular dependency of the temporal and spectral features in the squid echoes was utilized explicitly in data- 
model comparison for the determination of dominant scattering mechanisms and the assessment of different models. In field experiments, angle-dependent echo features can be collected using bistatic or multistatic sensing systems, or through repeated insonifications of the same scatterer or set of scatterers from different angles of orientation using monostatic systems (Jaffe and Roberts, 2011; Roberts and Jaffe, 2007, 2008). These features, and the sequential information embedded in the variation of echoes across multiple orientations, can be incorporated into the model formulation and provide additional dimensions of information for successful echo interpretation.

\section{Stationarity in echo data}

Another important topic that has not been investigated in depth in the context of echo interpretation is the stationarity in the echo data selected for analysis. Generally speaking, when an analysis window is selected from the echogram, it is assumed that the scattering processes in the volume enclosed by the analysis window are invariant during the time spanned between the first and last insonifications included such that the echo signals are stationary. The existence of stationarity ensures that spectrum estimated using these data is meaningful and the spectral features can be used quantitatively to infer the properties of the scattering sources through methods such as those introduced in Sec. 1.3.4 or those used in Sec. 4.4.2. However, in practice, the analysis windows are usually selected visually and subjectively without formal test of stationarity, which impairs the reliability of the result of echo interpretation. Similar problem is also present for inference conducted using echo statistics methods, since the assumption of the invariance of scattering processes is fundamental in the derivation of echo pdf from the data (see Sec. 4.3.3). The methods proposed by Borgnat et al. (2010) and other methods reviewed in that study provide several possibilities to tackle this problem. An important concept introduced by Borgnat et al. (2010) that may be particularly useful for stationarity analysis of echoes from marine organisms is "stationary relatively to the observation scale", which conveniently provide an avenue to deal with the patchy nature of biological aggregations in the ocean (Folt and Burns, 1999). 


\section{Application of Bayesian inference in echo interpretation}

In addition to the above discussion that focuses primarily on the analysis of echo features, a distinct and promising future research direction involves the use of Bayesian statistics in the formulation of echo interpretation. Different from the frequentist approach in which unknown parameters are often treated as having fixed values, in the Bayesian approach, unknown parameters are described by probability distributions. The Bayesian approach is advantageous because it provides a quantitative way to incorporate empirical or groundtruth information of both the desired and nuisance model parameters into the inference framework in the form of prior probabilities. Examples of prior information include local knowledge of the spatial and temporal occurrence pattern of different organisms, as well as groundtruth information from net catches and optical images collected concurrently or at nearby locations along with the acoustic data. Under the Bayesian framework, information gained in the observations is used to update the probability distribution of unknown parameters, and the distribution of the desired parameters can be conveniently obtained by marginalizing over the values of nuisance parameters (Box and Tiao, 1992).

\subsection{Broader impacts}

The new data, models, and approaches presented in this thesis can serve as the basi s for improving the accuracy and reliability of active acoustic survey techniques for collecting biological information such as the abundance and distribution patterns of different organisms, as well as the taxonomic composition of biological aggregations in the ocean. Active acoustic survey techniques are advantageous over the net-based and optical methods in their ability to observe these important biological quantities in a synoptic manner across relevant spatial and temporal scales at high resolution. This is crucial for comprehensive studies of the marine ecosystems, which are essential to the development of sustainable and ecosystem-based fisheries management.

Furthermore, although this research is inspired by the need for active acoustic techniques for the remote observation of marine organisms, the echo statistics models 
developed here are generally applicable in a broader context in the analysis of echoes from a variety of sources in different environments measured through other types of remote-sensing systems, such as the scattering of electromagnetic waves observed by radar. This generality is a direct benefit from the use of the physics-based approach to model development, since any parameter specific to the sensing system and the scatterers are explicit in the model formulation and can be adjusted adaptively according to the experimental scenario. This is an important improvement from most conventional data-driven echo statistics models whose usage is usually limited in time and space due to their system- and environment-specific properties.

More broadly, the insights gained from this study have implications in the studies of the echolocation of bats and toothed whales, particularly in the aspects of feature extraction from biosonar echoes. Specifically, the transmission, reception, and processing of echoes by the animals are determined by the physiology of biological structures, while at the same time the animals possess great maneuverabilities with respect to the scatterers that are currently not available for most human-made remote-sensing systems. In contrast to typical experimental scenarios for humanmade remote-sensing systems where a large number of repeated insonifications on aggregations of scatterers are collected, echolocating animals usually focus on individual prey and are subject to a limited number of biosonar emissions before capturing or missing the prey. Application of the techniques and approaches from this research may bring new ideas to the analysis of biosonar echoes to understand the processes involved in the detection, recognition, and tracking of prey. Based on the superior performance of biosonar systems over human-made remote-sensing system in these aspects, results of the biosonar studies can in turn feedback to guide the development of more accurate and reliable echo interpretation methods. 


\section{Appendix A}

\section{Normalization of echo pdfs}

The normalization procedure removes the echo pdfs' dependency on the absolute scattering amplitudes of the scatterers and other environment and system-specific parameters, such as range-dependent transmission loss and system gain. This enables direct analysis of the aggregation composition using of a set of standard normalized curves. For a set of discrete echo samples, the echo amplitude of each sample is normalized by the rms amplitude of all samples which is calculated by:

$$
A_{r m s}=\sqrt{\sum x_{q}^{2} / N},
$$

where $x_{q}$ denotes the $q$ th sample out of a total of $N$ samples. For a given pdf, $p_{X}(x)$, each point on the amplitude axis is normalized by the rms amplitude of the pdf,

$$
A_{r m s}=\sqrt{\int x^{2} p_{X}(x) d x} .
$$

The effect of normalization on the echo pdf is demonstrated (Fig. A-1). In this illustration, theoretical echo pdfs of several one-component aggregations are calculated using (3.10) and (3.13) with $M=1$ and different values of $N$ (=N in Fig. A-1). The predicted curves are normalized according to (A.2). 

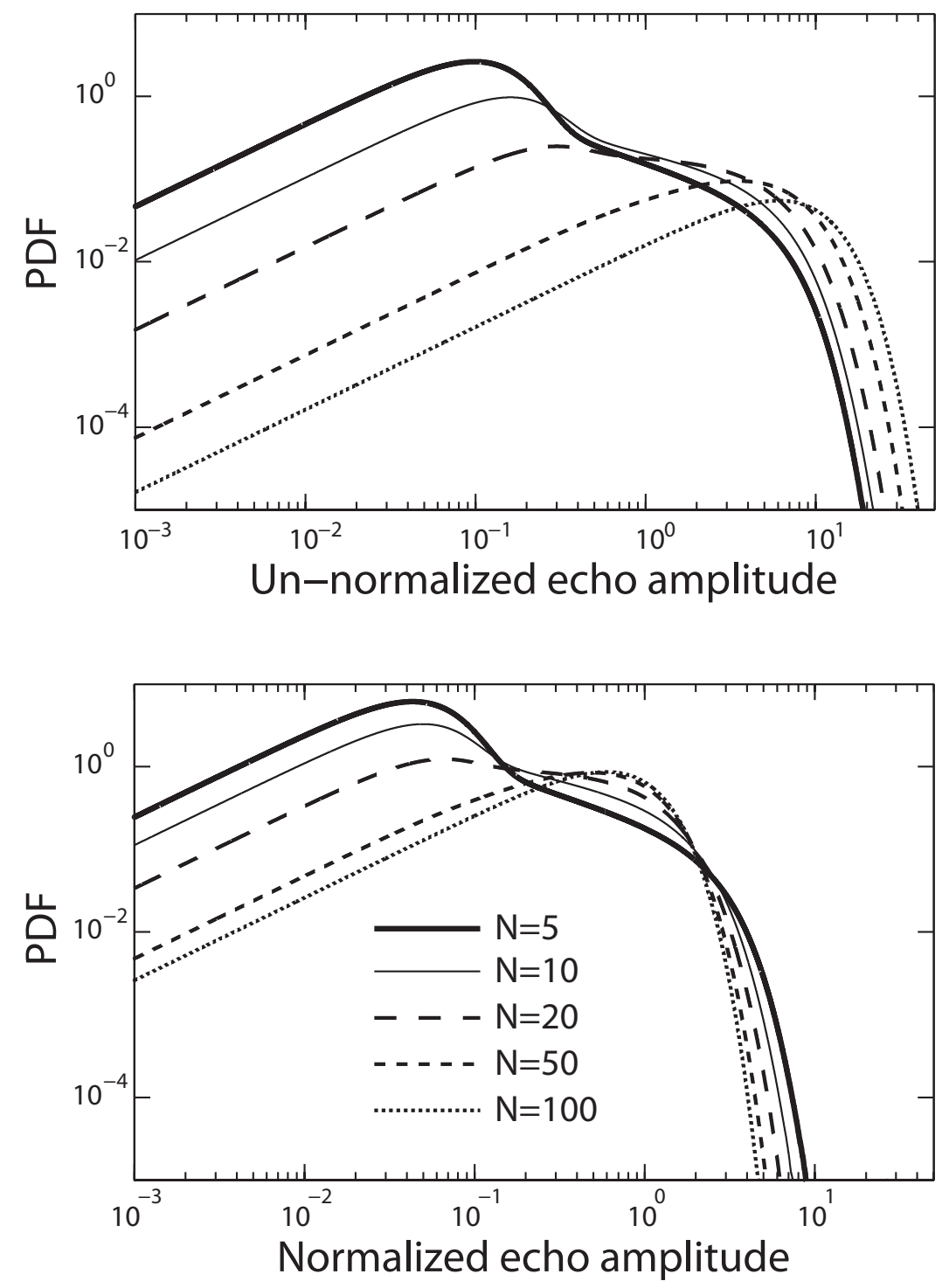

Figure A-1: Comparison of several un-normalized (top) and normalized (bottom) echo pdfs calculated using the CF-based echo pdf formula with varying numbers of only one type of scatterer $(N)$. 


\section{Appendix B}

\section{Addition of noise to echo pdf}

\section{models}

The echo pdf models developed or used in this thesis are "noise-free" models in which only the scattering mechanisms of the scatterers and the influence of the sensing systems are considered in the physics-based modeling. However, the presence of noise is expected to affect the statistics of echoes collected in both laboratory and field experiments and must be incorporated in data analysis. Specifically, noise adds additional complex random components to the echoes from scatterers, and is expected to result in a slight "whitening" of the echoes and cause any non-Rayleigh characteristics to tend slightly toward Rayleigh (Stanton and Chu, 2010). To account for this potential effect, random noise is generated and added to the echo pdf models used for echo statistics analysis of experimental data presented in Sec. 2.5 and Chapter 4. The procedure is discussed as follows.

The echo pdf of background noise in the laboratory squid backscattering experi-

ments (Chapter 2) shows strong Rayleigh-like features [Fig. B-1(a)], as expected from random complex noise with both the real and imagenary parts following the Gaussian distribution. In this figure, both the squid echoes and the noise are normalized to the rms amplitude of the squid echo samples following in the procedure in Appendix A. Rayleigh distributions with different power parameters $\left(\lambda_{R}\right)$ are plotted along with the pdf of squid echoes and noise to facilitate the comparison [Fig. B-1(b)]. The Rayleigh 
distribution is shown below in order to avoid confusion among different conventions:

$$
f_{R}\left(x ; \lambda_{R}\right)=\frac{x}{\lambda_{R}^{2}} e^{-x^{2} / 2 \lambda_{R}^{2}}
$$

According to the Rayleigh-like features of the noise echo pdf, Gaussian-distributed noise with a standard deviation $\sigma_{\mathrm{n}}=1 / \sqrt{2} r_{\mathrm{fn}}$ is generated, where $r_{\mathrm{fn}}$ is the ratio between the rms amplitudes of the fish echoes and the rms amplitude of noise. This noise is added coherently to each realization of the ensemble of the numerically simulated echoes from a randomly-oriented, randomly-rough prolate spheroid (see Sec. 2.5.2), from which the noise-added echo pdf model is derived using KDE (see Sec. 2.5 for the detail of KDE). As a result of normalization, the power parameters of the Rayleigh pdfs are $\lambda_{\mathrm{R}}=1$ and $\lambda_{\mathrm{R}}=1 / r_{\mathrm{fn}}$ for the squid echoes and noise, respectively.

Due to the high level of noise in this data set (approximately one order of magnitude lower than the squid echoes), prominent "whitening" effect of the noise is observed on the resultant echo pdf models [Fig. B-1(b)]. Note that the noise threshold shown here is $6 \mathrm{~dB}$ higher than the actual background noise level for the purpose of data quality control as described in detail in Sec. 2.2.5.

A similar procedure is used to generate and add noise into echo pdf models used in the analysis of the statistics of broadband echoes from fish collected in the ocean (Chapter 4). An analysis window for background noise is identified by visually selecting regions on the echogram that are close to the target fish aggregation and at approximately the same range from the sonar, but with much lower echo returns [Fig. B-2(a)]. Both the fish echoes and the noise are normalized with respect to the rms amplitude of the fish echo samples (Appendix A). Analogous to the procedure described above for the case of squid scattering, the ratio between the rms amplitude of the fish echoes and the noise, $r_{\mathrm{fn}}$, is used to generated Gaussian-distributed noise with a standard deviation $\sigma_{\mathrm{n}}=1 / \sqrt{2} r_{\mathrm{fn}}$. The noise is added to the simulated time-series before echo envelopes are taken (see Sec. 4.2). The above is done under the assumption that the envelope amplitude of the noise is Rayleigh-distributed. Although the observed echo pdf of noise has a strong non-Rayleigh tail [Fig. B-2(b)], this is likely 
(b)
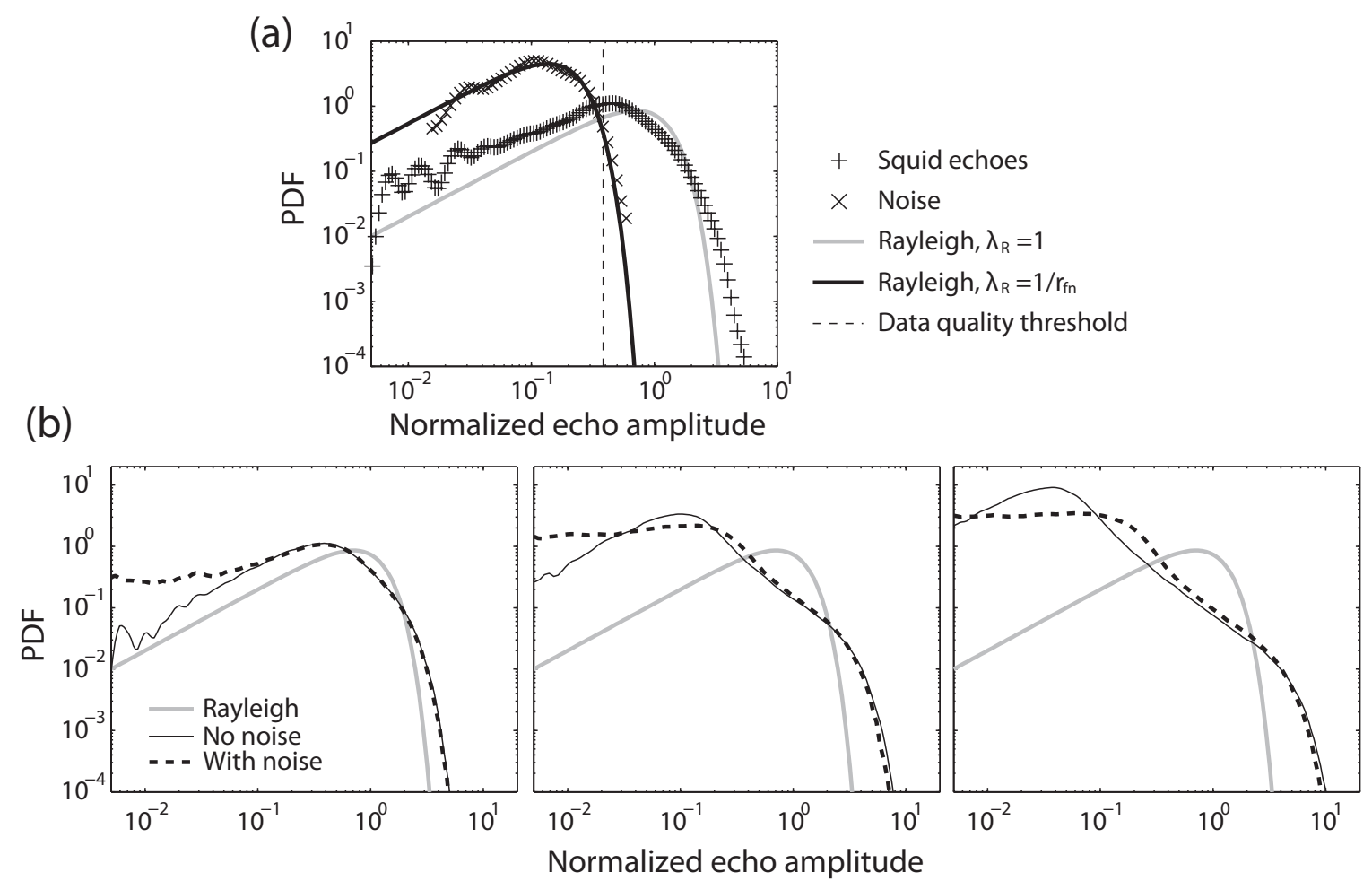

Figure B-1: (a) Comparison of the pdfs of squid echoes and background noise, as well as their corresponding Rayleigh pdfs (see text). (c) Comparison of rough prolate spheroid echo pdf model's generated with and without added noise. 
(a)

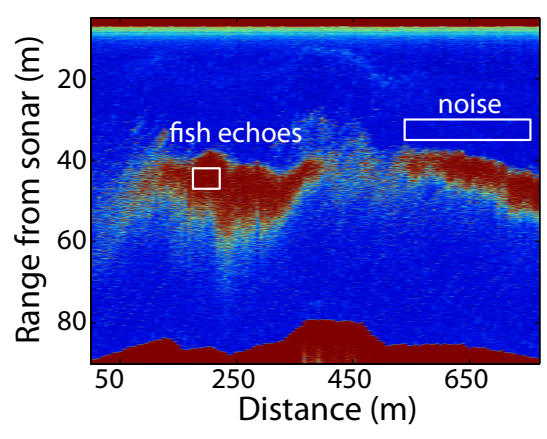

(b)

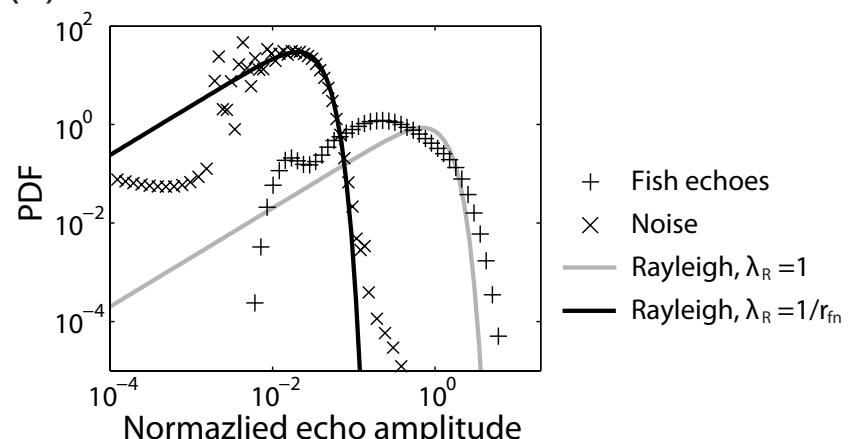

(c)

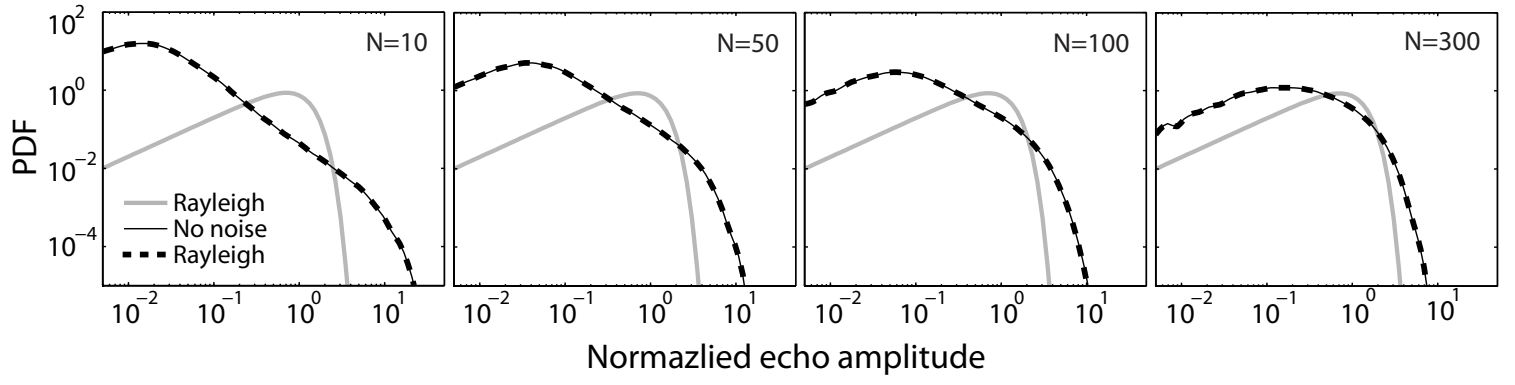

Figure B-2: (a) Echogram of the fish aggregation with the analysis windows for fish echoes and background noise. Note that the depth of the echosounder is not corrected in this echogram so that the bottom appears to undulate as opposed to the flat bottom shown in Fig. 4-11. (b) Comparison of the pdfs of fish echoes and background noise, as well as their corresponding Rayleigh pdfs (see text). (c) Comparison of broadband echo pdf model's generated with and without added noise.

caused by occasionally insonification of fish within the noise analysis window, given its proximity to the fish aggregations. The Rayleigh-like shape of the noise echo pdf except for the tail region is used to justify the use of the above procedure.

The broadband echo pdf models generated with and without the addition of noise are nearly identical [Fig. B-2(c)]. This is expected, since the amplitude of added noise is two orders of magnitude lower than that of the simulated fish echoes, as opposed to the case of squid scattering where a higher level of noise was observed [Fig. B-1(a)]. Note it is possible that the noise may not be Rayleigh-distributed with a heavier tail. In such cases, the contamination of noise will cause the model echo pdfs to tend toward the Rayleigh distribution and result in underestimation of the numerical density of fish (Sec. 4.4.4). 


\section{Bibliography}

Abraham, D. A. and Lyons, A. P. Novel physical interpretations of $K$-distributed reverberation. IEEE J. Oceanic Eng., 27(4):800-813, 2002.

Abraham, D. A. and Lyons, A. P. Reverberation envelope statistics and their dependence on sonar bandwidth and scattering patch size. IEEE J. Oceanic Eng., 29(1): 126-137, 2004.

Abraham, D. A. and Lyons, A. P., Eds. Special issue on Non-Rayleigh reverberation and clutter, volume 75. IEEE J. Oceanic Eng., 2010.

Abraham, D. A., Gelb, J. M., and Oldag, A. W. K-Rayleigh mixture model for sparse active sonar clutter. In OCEANS 2010 IEEE - Sydney, pages 1-6, 2010.

Abraham, D. A., Gelb, J. M., and Oldag, A. W. Background and clutter mixture distributions for active sonar statistics. IEEE J. Oceanic Eng., 36(2):231-247, 2011.

Ainslie, M. A. and Leighton, T. G. Review of scattering and extinction cross-sections, damping factors, and resonance frequencies of a spherical gas bubble. J. Acoust. Soc. Am., 130(5):3184-3208, 2011.

Anderson, C. I. H., Horne, J. K., and Boyle, J. Classifying multi-frequency fisheries acoustic data using a robust probabilistic classification technique. J. Acoust. Soc. Am., 121(6):EL230-EL237, 2007.

Arnaya, I. N. and Sano, N. Studies on acoustic target strength of squid VI. simulation of squid target strength by prolate spheroid model. Bull. Fac. Fish. Hokkaido Univ., 41(1):32-42, 1990 .

Arnaya, I. N., Sano, N., and Iida, K. Studies on acoustic target strength of squid II. effect of behaviour on averaged dorsal aspect target strength. Bull. Fac. Fish. Hokkaido Univ., 40(2):83-99, 1989.

Au, W. W. L. The Sonar of Dolphins. Springer, 1993.

Au, W. W. L. and Benoit-Bird, K. J. Broadband backscatter from individual Hawaiian mesopelagic boundary community animals with implications for spinner dolphin foraging. J. Acoust. Soc. Am., 123(5):2884-2894, 2008. 
Azzalini, A. Statistical Inference Based on the Likelihood. Chapman \& Hall/CRC, 1996.

Barakat, R. First-order statistics of combined random sinusoidal waves with applications to laser speckle patterns. Optica Acta: Int'l J. Optics, 21(11):903-921, 1974.

Benoit-Bird, K. J. Dynamic 3-dimensional structure of thin zooplankton layers is impacted by foraging fish. Mar. Eco. Prog. Ser., 396:61-76, 2009a.

Benoit-Bird, K. J. The effects of scattering-layer composition, animal size, and numerical density on the frequency response of volume backscatter. ICES J. Mar. Sci., 66(3):582-593, 2009b.

Benoit-Bird, K. J. and Au, W. W. L. Spatial dynamics of a nearshore, micronekton sound-scattering layer. ICES J. Mar. Sci., 60(4):899-913, 2003.

Benoit-Bird, K. J., Gilly, W. F., Au, W. W. L., and Mate, B. Controlled and in situ target strengths of the jumbo squid Dosidicus gigas and identification of potential acoustic scattering sources. J. Acoust. Soc. Am., 123(3):1318-1328, 2008.

Bevington, P. R. and Robinson, D. K. Data Reduction and Error Analysis for the Physical Sciences. McGraw-Hill, 2003.

Bhatia, S., Stanton, T. K., and Baik, K. Non-Rayleigh scattering by a randomly oriented elogated scatterer randomly located in a beam. IEEE J. Oceanic Eng.,, submitted.

Borgnat, P., Flandrin, P., Honeine, P., Richard, C., and Xiao, J. Testing stationarity with surrogates: A time-frequency approach. IEEE Transactions on Signal Processing, 58(7):3459 -3470, 2010.

Botev, Z. I., Grotowski, J. F., and Kroese, D. P. Kernel density estimation via diffusion. Ann. Stat., 38(5):2916-2957, 2010.

Box, G. E. P. and Tiao, G. C. Bayesian Inference in Statistical Analysis. WileyInterscience, reprint edition, 1992.

Boyle, P. R. and Rodhouse, P. Cephalopods: Ecology and Fisheries. Wiley-Blackwell, 2005.

Brierley, A. S., Axelsen, B. E., Boyer, D. C., Lynam, C. P., Didcock, C. A., Boyer, H. J., Sparks, C. A. J., Purcell, J. E., and Gibbons, M. J. Single-target echo detections of jellyfish. ICES J. Mar. Sci., 61(3):383-393, 2004.

Chapman, R. P. and Marshall, J. R. Reverberation from deep scattering layers in the western North Atlantic. J. Acoust. Soc. Am., 40(2):405-411, 1966. 
Chapman, R. P., Bluy, O. Z., Adlington, R. H., and Robison, A. E. Deep scattering layer spectra in the Atlantic and Pacific Oceans and adjacent seas. J. Acoust. Soc. Am., 56(6):1722-1734, 1974.

Chib, S., Heckman, J., and Leamer, E. Markov chain Monte Carlo methods: computation and inference. In Handbook of econometrics, volume 5, pages 3569-3649. Elsevier, 2001.

Chotiros, N. P. Non-Rayleigh distributions in underwater acoustic reverberation in a patchy environment. IEEE J. Oceanic Eng., 35(2):236-241, 2010.

Chu, D. and Stanton, T. K. Statistics of echoes from a directional sonar beam insonifying finite numbers of single scatterers and patches of scatterers. IEEE J. Oceanic Eng., 35(2):267-277, 2010.

Chu, D. and Stanton, T. K. Application of pulse compression techniques to broadband acoustic scattering by live individual zooplankton. J. Acoust. Soc. Am., 104(1):39$55,1998$.

Chu, D., Foote, K. G., and Stanton, T. K. Further analysis of target strength measurements of Antarctic krill at 38 and $120 \mathrm{kHz}$ : comparison with deformed cylinder model and inference of orientation distribution. J. Acoust. Soc. Am., 93(5):29852988, 1993.

Chu, D., Wiebe, P., and Copley, N. Inference of material properties of zooplankton from acoustic and resistivity measurements. ICES J. Mar. Sci., 57(4):1128-1142, 2000 .

Chu, D., Stanton, T. K., Jech, J. M., and Reeder, D. B. Modeling of the backscattering by swimbladder-bearing fish. J. Acoust. Soc. Am., 120(5):3105-3105, 2006.

Clay, C. S. and Heist, B. G. Acoustic scattering by fish-Acoustic models and a two-parameter fit. J. Acoust. Soc. Am., 75(4):1077-1083, 1984.

Clay, C. S. and Horne, J. K. Acoustic models of fish: The Atlantic cod (Gadus morhua). J. Acoust. Soc. Am., 96(3):1661-1668, 1994.

Clifford, L., Fitzgerald, P., and James, D. Non-Rayleigh first-order statistics of ultrasonic backscatter from normal myocardium. Ultrasound Med. Biol., 19(6):487-495, 1993.

Conover, W. J. Practical Nonparametric Statistics. Wiley, 3rd edition, 1999.

Crowther, P. A. Fluctuation Statistics of Sea-bed Acoustic Backscatter. New York: Plenum, 1980.

De Robertis, A. Small-scale spatial distribution of the euphausiid Euphausia pacifica and overlap with planktivorous fishes. J. Plankton Res., 24(11):1207-1220, 2002. 
De Robertis, A., McKelvey, D. R., and Ressler, P. H. Development and application of an empirical multifrequency method for backscatter classification. Can. J. Fish. Aquat. Sci, 67(9):1459-1474, 2010.

Denbigh, P. and Smith, Q. Determination of fish number density by a statistical analysis of backscattered sound. J. Acoust. Soc. Am., 90(1):457, 1991.

Diachok, O. Bioacoustic absorption spectroscopy: a new approach to monitoring the number and lengths of fish in the ocean. In Medwin, H., editor, Sounds in the sea: from ocean acoustics to acoustical oceanography, pages 398-410. University Press, Cambridge, UK, 2005.

Diachok, O. Effects of absorptivity due to fish on transmission loss in shallow water. J. Acoust. Soc. Am., 105(4):2107-2128, 1999.

Ding, L. Direct laboratory measurement of forward scattering by individual fish. $J$. Acoust. Soc. Am., 101(6):3398-3404, 1997.

Dragonette, L. R., Numrich, S. K., and Frank, L. J. Calibration technique for acoustic scattering measurements. J. Acoust. Soc. Am., 69(4):1186-1189, 1981.

Ehrenberg, J. E. A method for extracting the fish target strength distribution from acoustic echoes. In Proc. 1972 IEEE conf. eng. ocean env., pages 61-64, 1972.

Ehrenberg, J. E. A review of target strength estimation techniques. Underwater Acoustic Data Processing. Kluwer Academic Publishers, Dordrecht, pages 161-175, 1989.

Ehrenberg, J. E. Recursive algorithm for estimating the spatial density of acoustic point scatterers. J. Acoust. Soc. Am., 56(2):542-547, 1974.

Farmer, D. M., Trevorrow, M. V., and Pedersen, B. Intermediate range fish detection with a 12-kHz sidescan sonar. J. Acoust. Soc. Am., 106:2481, 1999.

Fässler, S. M. M., Fernandes, P. G., Semple, S. I. K., and Brierley, A. S. Depthdependent swimbladder compression in herring Clupea harengus observed using magnetic resonance imaging. J. Fish Biol., 74(1):296-303, 2009a.

Fässler, S. M. M., Brierley, A. S., and Fernandes, P. G. A Bayesian approach to estimating target strength. ICES J. Mar. Sci., 66(6):1197-1204, 2009b.

Fässler, S. M., Gorska, N., Ona, E., and Fernandes, P. G. Differences in swimbladder volume between Baltic and Norwegian spring-spawning herring: Consequences for mean target strength. Fish. Res., 92(23):314-321, 2008.

Feuillade, C. and Nero, R. W. A viscous-elastic swimbladder model for describing enhanced-frequency resonance scattering from fish. J. Acoust. Soc. Am., 103(6): $3245-3255,1998$. 
Feuillade, C., Nero, R. W., and Love, R. H. A low-frequency acoustic scattering model for small schools of fish. J. Acoust. Soc. Am., 99(1):196-208, 1996.

Folt, C. L. and Burns, C. W. Biological drivers of zooplankton patchiness. Trends Ecol. Evol., 14:300-305, 1999.

Foote, K. G. Extinction cross-section of norwegian spring-spawning herring. ICES J. Mar. Sci., 56(5):606-612, 1999.

Foote, K. G. Optimizing two targets for calibrating a broadband multibeam sonar. In OCEANS 2006, pages 1-4, 2006.

Foote, K. G., Atkins, P. R., Bongiovanni, C. C., Francis, D. T., Eriksen, P. K., Larsen, M., and Mortensen, T. Measuring the frequency response function of a seven octave bandwidth echo sounder. Proceedings-Institute of Acoustics, 21(1):88-95, 1999a.

Foote, K. G., Atkins, P. R., Francis, D. T. I., and Knutsen, T. Measuring echo spectra of marine organisms over a wide bandwidth. In Proceedings of the International Conference on Underwater Acoustic Measurements: Technologies and Results, II, Heraklion, Greece, volume 28, pages 501-508, 2005.

Foote, K. G. Linearity of fisheries acoustics, with addition theorems. J. Acoust. Soc. Am., 73(6):1932-1940, 1983.

Foote, K. G. Rather-high-frequency sound scattering by swimbladdered fish. J. Acoust. Soc. Am., 78(2):688-700, 1985.

Foote, K. G. Correcting acoustic measurements of scatterer density for extinction. J. Acoust. Soc. Am., 88(3):1543-1546, 1990.

Foote, K. G. Acoustic sampling volume. J. Acoust. Soc. Am., 90(2):959-964, 1991.

Foote, K. G. and Francis, D. T. I. Comparing Kirchhoff-approximation and boundaryelement models for computing gadoid target strengths. J. Acoust. Soc. Am., 111 (4):1644-1654, 2002.

Foote, K. G., Ona, E., and Toresen, R. Determining the extinction cross section of aggregating fish. J. Acoust. Soc. Am., 91(4):1983-1989, 1992.

Foote, K. G., Knutsen, T., Atkins, P. R., Bongiovanni, C., Francis, D. T. I., Eriksen, P. K., and Mortensen, T. A seven-octave-bandwidth echo sounding system for application to fish and zooplankton. J. Acoust. Soc. Am., 105(2):994, 1999b.

Foote, K. G., Knutsen, T., Atkins, P. R., Bongiovanni, C., Francis, D. T. I., Eriksen, P. K., Larsen, M. T., and Mortensen, T. Broadband echo spectra from euphausiids and copepods. J. Acoust. Soc. Am., 108(5):2469, 2000.

Francis, D. T. I. and Foote, K. G. Depth-dependent target strengths of gadoids by the boundary-element method. J. Acoust. Soc. Am., 114(6):3136-3146, 2003. 
Gallaudet, T. C. and de Moustier, C. P. High-frequency volume and boundary acoustic backscatter fluctuations in shallow water. J. Acoust. Soc. Am., 114(2):707-725, 2003.

Gauss, R. C., Fialkowski, J. M., Kunz, E. L., Menis, R., Stanton, T. K., Sellers, C. J., and Jech, J. M. Clutter variability due to fish aggregations: Mid-frequency measurements in the gulf of maine. In Proceedings of the 3rd International Conference and Exhibition on Underwater Acoustic Measurements: Technologies and Results, edited by JS Papadakis and L. Bjorno (Nafplion, Peloponnese, Greece, 2009), pages 459-466, 2009.

Gelb, J. M., Heath, R. E., and Tipple, G. L. Statistics of distinct clutter classes in midfrequency active sonar. IEEE J. Oceanic Eng., 35(2):220-229, 2010.

Goodman, J. W. Statistical Optics. Wiley-Interscience, 1st edition, 1985.

Gorska, N. and Ona, E. Modelling the acoustic effect of swimbladder compression in herring. ICES J. Mar. Sci., 60(3):548-554, 2003a.

Gorska, N. and Ona, E. Modelling the effect of swimbladder compression on the acoustic backscattering from herring at normal or near-normal dorsal incidences. ICES J. Mar. Sci., 60(6):1381-1391, 2003b.

Goss, C., Middleton, D., and Rodhouse, P. Investigations of squid stocks using acoustic survey methods. Fish. Res., 54(1):111-121, 2001.

Greene, C. H., Stanton, T. K., Wiebe, P. H., and McClatchie, S. Acoustic estimates of Antarctic krill. Nature, 349(6305):110-110, 1991.

Greenlaw, C. F. Acoustical estimation of zooplankton populations. Limnol. Oceanogr., 24(2):226-242, 1979.

Greig-Smith, P. Quantitative Plant Ecology. University of California Press, 1984.

Griffin, D. R. Listening in the Dark. Yale University Press, 1958.

Hall, M. Measurements of acoustic volume backscattering in the Indian and Southern Oceans. Mar. Fresh. Res., 32(6):855-876, 1981.

Hall, M. and Quill, A. Biological sound scattering in an ocean eddy. Mar. Fresh. Res., 34(4):563-572, 1983.

Hanlon, R. T., Maxwell, M. R., Shashar, N., Loew, E. R., and Boyle, K. L. An ethogram of body patterning behavior in the biomedically and commercially valuable squid Loligo pealei off Cape Cod, Massachusetts. Biol. Bull., 197(1):49-62, 1999.

Hanlon, R. T. and Messenger, J. B. Cephalopod Behaviour. Cambridge University Press, 1998. 
Harris, R., Wiebe, P., Lenz, J., Skjoldal, H.-R., and Huntley, M., editors. ICES Zooplankton Methodology Manual. Academic Press, 1st edition, 2000.

Hazen, E. L. and Horne, J. K. A method for evaluating the effects of biological factors on fish target strength. ICES J. Mar. Sci., 60(3):555-562, 2003.

Heezen, B. C. and Hollister, C. D. The Face of the Deep. Oxford University Press, 1971.

Hersey, J., Backus, R. H., and Hellwig, J. Sound-scattering spectra of deep scattering layers in the western north atlantic ocean. Deep Sea Res., 8(34):196-210, 1961.

Higginbottom, I. R., Pauly, T. J., and Heatley, D. C. Virtual echograms for visualization and post-processing of multiple-frequency echosounder data. In Proceedings of the Fifth European Conference on Underwater Acoustics, ECUA, pages 1497-1502, 2000 .

Holliday, D. V. Resonance structure in echoes from schooled pelagic fish. J. Acoust. Soc. Am., 51(4B):1322-1332, 1972.

Holliday, D. V., Pieper, R. E., and Kleppel, G. S. Determination of zooplankton size and distribution with multifrequency acoustic technology. ICES J. Mar. Sci., 46 (1):52-61, 1989 .

Holliday, D. V. Extracting bio-physical information from the acoustic signatures of marine organisms. In Anderson, N. R. and Zahuranec, B. J., editors, Oceanic sound scattering prediction, pages 619-624. Plenum Press, New York, 1977.

Horne, J. K., Sawada, K., Abe, K., Kreisberg, R. B., Barbee, D. H., and Sadayasu, K. Swimbladders under pressure: anatomical and acoustic responses by walleye pollock. ICES J. Mar. Sci., 66(6):1162-1168, 2009.

Iida, K., Takahashi, R., Tang, Y., Mukai, T., and Sato, M. Observation of marine animals using underwater acoustic camera. Jpn. J. Appl. Phys. Part 1, 45(5B): 4875-4881, 2006.

Imaizumi, T., Furusawa, M., Akamatsu, T., and Nishimori, Y. Measuring the target strength spectra of fish using dolphin-like short broadband sonar signals. J. Acoust. Soc. Am., 124(6):3440-3449, 2008.

Ito, M., Matsuo, I., Imaizumi, T., Akamatsu, T., Wang, Y., and Nishimori, Y. Temporal and spectral feature extraction from fish school using a broadband split-beam echosounder. J. Acoust. Soc. Am., 130(4):2336-2336, 2011.

Jaffe, J. S. and Roberts, P. L. D. Estimating fish orientation from broadband, limitedangle, multiview, acoustic reflections. J. Acoust. Soc. Am., 129(2):670-680, 2011.

Jakeman, E. and Pusey, P. A model for non-Rayleigh sea echo. IEEE Trans. Antenn. Prop., 24(6):806-814, 1976. 
Jansson, P.-A. Acoustic scattering from a rough sphere. J. Acoust. Soc. Am., 93(6): 3032-3042, 1993.

Jech, J. M. and Michaels, W. L. A multifrequency method to classify and evaluate fisheries acoustics data. Can. J. Fish. Aqua. Sci., 63(10):2225-2235, 2006.

Jech, J. M. Interpretation of multi-frequency acoustic data: Effects of fish orientation. J. Acoust. Soc. Am., 129(1):54-63, 2011.

Jech, J. M. and Horne, J. K. Three-dimensional visualization of fish morphometry and acoustic backscatter. Acoust. Res. Lett. Online, 3(1):35-40, 2002.

Jech, J. M., Horne, J. K., and Schael, D. M. Sensitivity of the inverse problem to size-class selection. J. Acoust. Soc. Am., 98(5):2901-2901, 1995.

Jobst, W. J. and Smits, T. I. Mathematical model for volume reverberation: experiment and simulation. J. Acoust. Soc. Am., 55(2):227-236, 1974.

Johnson, M. L. Orientation dependence of the acoustic backscatter for elongated zooplankton. PhD thesis, MIT-WHOI Joint Program, Applied Ocean Science and Engineering, Cambridge, MA, 1993.

Jones, B. A. Echo statistics of aggregations of scatterers in a random waveguide: Application to biologic sonar clutter. PhD thesis, Naval Postgraduate School, Monterey, CA, 2012.

Jones, B. A., Lavery, A. C., and Stanton, T. K. Use of the distorted wave Born approximation to predict scattering by inhomogeneous objects: Application to squid. J. Acoust. Soc. Am., 125(1):73-88, 2009.

Jones, C. D. and Jackson, D. R. Midfrequency backscatter imaging of fish schools in a shallow water waveguide. J. Acoust. Soc. Am., 125:2550, 2009.

Kajiwara, Y., Iida, K., and Kamei, Y. Measurement of target strength for the flying squid (Ommastrephes bartrami). Bull. Fac. Fish. Hokkaido Univ., 41(4):205-212, 1990.

Kang, D., Mukai, T., Iida, K., Hwang, D., and Myoung, J. The influence of tilt angle on the acoustic target strength of the Japanese common squid (Todarodes pacificus). ICES J. Mar. Sci., 62(4):779-789, 2005.

Kang, D., Iida, K., Mukai, T., and Kim, J. Density and sound speed contrasts of the Japanese common squid Todarodes pacificus and their influence on acoustic target strength. Fish. Sci., 72(4):728-736, 2006.

Kargl, S. G. and Marston, P. L. Observations and modeling of the backscattering of short tone bursts from a spherical shell: Lamb wave echoes, glory, and axial reverberations. The J. Acoust. Soc. Am., 85(3):1014-1028, 1989. 
Kawabata, A. Measurement of the target strength of Japanese flying squid, Todarodes pacificus Steenstrup. Bull. Tohoku Reg. Fish. Res. Lab., (61):29-40, 1999.

Kawabata, A. Measurement of the target strength of live squid, Todarodes pacificus Steenstrup, in controlled body tilt angle. Bull. Tohoku Reg. Fish. Res. Lab., (64): 61-67, 2001.

Kawabata, A. Target strength measurements of suspended live ommastrephid squid, Todarodes pacificus, and its application in density estimations. Fish. Sci., 71:63-72, 2005.

Kay, S. M. Fundamentals of Statistical Signal Processing: Estimation Theory. Prentice Hall, 1993.

Klemas, V. Fisheries applications of remote sensing: An overview. Fish. Res., 2012.

Kullback, S. Statistics and Information Theory. J. Wiley and Sons, New York, 1959.

La Cour, B. Statistical characterization of active sonar reverberation using extreme value theory. IEEE J. Oceanic Eng., 29(2):310-316, 2004.

Lalli, C. M. and Parsons, T. R. Biological Oceanography: An Introduction. Butterworth-Heinemann, 1997.

Lange, A. M. T. and Johnson, K. L. Dorsal mantle length-total weight relationships of squids Loligo pealei and Illex illecebrosus from the Atlantic coast of the United States. NOAA Technical Report NMFS SSRF-745, 1981.

Lavery, A. C., Chu, D., and Moum, J. N. Observations of broadband acoustic backscattering from nonlinear internal waves: Assessing the contribution from microstructure. IEEE J. Oceanic Eng., 35(4):695 -709, 2010a.

Lavery, A. C. and Ross, T. Acoustic scattering from double-diffusive microstructure. J. Acoust. Soc. Am., 122(3):1449-1462, 2007.

Lavery, A. C., Stanton, T. K., McGehee, D. E., and Chu, D. Three-dimensional modeling of acoustic backscattering from fluid-like zooplankton. J. Acoust. Soc. Am., 111(3):1197-1210, 2002.

Lavery, A. C., Chu, D., and Moum, J. N. Measurements of acoustic scattering from zooplankton and oceanic microstructure using a broadband echosounder. ICES J. Mar. Sci., 67(2):379-394, 2010b.

Lavery, A. C., Lawson, G. L., Wiebe, P. H., Stanton, T. K., Fincke, J. R., and Copley, N. J. Acoustic characterization of thecosome pteropods and recent field measurements in the context of ocean acidification. J. Acoust. Soc. Am., 132(3): 1882-1882, 2012. 
Lawson, G. L., Wiebe, P. H., Ashjian, C. J., Chu, D., and Stanton, T. K. Improved parametrization of Antarctic krill target strength models. J. Acoust. Soc. Am., 119 (1):232-242, 2006.

Lawson, G. L., Lavery, A. C., Wiebe, P. H., Fincke, J. R., and Copley, N. J. Recent development and application of active acoustic techniques for studies of zooplankton ecology and implications for ocean observatories. J. Acoust. Soc. Am., 132(3): 1915-1915, 2012.

Le Chevalier, F. Principles of Radar and Sonar Signal Processing. Artech House, Boston, 2002.

Lee, W.-J. and Stanton, T. K. Statistics of echoes from mixed assemblages of scatterers with different scattering amplitudes and numerical densities. IEEE J. Oceanic Eng., submitted.

Lee, W.-J., Lavery, A. C., and Stanton, T. K. Interpretation of the compressed pulse output for broadband acoustic scattering from inhomogeneous weakly scattering objects. J. Acoust. Soc. Am., 128(4):2460, 2010.

Lee, W.-J., Lavery, A. C., and Stanton, T. K. Orientation dependence of broadband acoustic backscattering from live squid. J. Acoust. Soc. Am., 131(6):4461-4475, 2012.

Leighton, T. G. The Acoustic Bubble. Academic Press, 1997.

Love, R. H. Dorsal-aspect target strength of an individual fish. J. Acoust. Soc. Am., 49(3B):816-823, 1971.

Love, R. H. Target strength of an individual fish at any aspect. J. Acoust. Soc. Am., 62(6):1397-1403, 1977.

Love, R. H. Resonant acoustic scattering by swimbladder-bearing fish. J. Acoust. Soc. Am., 64(2):571-580, 1978.

MacLennan, D. N. and Menz, A. Interpretation of in situ target-strength data. ICES J. Mar. Sci., 53(2):233-236, 1996.

Madsen, P. T., Wilson, M., Johnson, M., Hanlon, R. T., Bocconcelli, A., de Soto, N. A., and Tyack, P. L. Clicking for calamari: toothed whales can echolocate squid Loligo pealeii. Aqua. Biol., 1:141-150, 2007.

Madureira, L. S. P., Everson, I., and Murphy, E. J. Interpretation of acoustic data at two frequencies to discriminate between Antarctic krill (Euphausia superba Dana) and other scatterers. J. Plankton Res., 15(7):787-802, 1993.

Makris, N. C., Ratilal, P., Symonds, D. T., Jagannathan, S., Lee, S., and Nero, R. W. Fish population and behavior revealed by instantaneous continental shelfscale imaging. Science, 311(5761):660-663, 2006. 
Mann, K. and Lazier, J. Dynamics of Marine Ecosystems: Biological-Physical Interactions in the Oceans. Wiley-Blackwell, 3rd edition, 2005.

Marston, P. L., Kargl, S. G., and Williams, K. L. Rayleigh, Lamb, and Whispering Gallery Wave Contributions to Backscattering from Smooth Elastic Objects in Water Described by a Generalization of GTD. Amsterdam, The Netherlands: Elsevier, 1990.

Martin, L. V., Stanton, T. K., Wiebe, P. H., and Lynch, J. F. Acoustic classification of zooplankton. ICES J. Mar. Sci., 53(2):217-224, 1996.

Martin-Traykovski, L. V., O’Driscoll, R. L., and McGehee, D. E. Effect of orientation on broadband acoustic scattering of Antarctic krill Euphausia superba: Implications for inverting zooplankton spectral acoustic signatures for angle of orientation. $J$. Acoust. Soc. Am., 104(4):2121-2135, 1998a.

Martin-Traykovski, L. V., Stanton, T. K., Wiebe, P. H., and Lynch, J. F. Modelbased covariance mean variance classification techniques: algorithm development and application to the acoustic classification of zooplankton. IEEE J. Oceanic Eng., 23(4):344-364, 1998b.

Matsuo, I., Imaizumi, T., Akamatsu, T., Furusawa, M., and Nishimori, Y. Analysis of the temporal structure of fish echoes using the dolphin broadband sonar signal. J. Acoust. Soc. Am., 126(1):444-450, 2009.

Matsuo, I., Ito, M., Imaizumi, T., Akamatsu, T., Wang, Y., and Nishimori, Y. Feature extraction for classification of fish species using the cepstral analysis. J. Acoust. Soc. Am., 132(3):1897-1897, 2012.

McClatchie, S., Alsop, J., and Coombs, R. F. A re-evaluation of relationships between fish size, acoustic frequency, and target strength. ICES J. Mar. Sci., 53(5):780-791, 1996.

Medwin, H. and Clay, C. S. Fundamentals of Acoustical Oceanography. Academic Press, San Diego, 1998.

Middleton, D. New physical-statistical methods and models for clutter and reverberation: the $K A$-distribution and related probability structures. IEEE J. Oceanic Eng., 24(3):261-284, 1999.

Mooney, T. A., Lee, W.-J., and Hanlon, R. T. Long-duration anesthetization of squid (Doryteuthis pealeii). Mar. Fresh. Behav. Phys., 43(4):297-303, 2010.

Morse, P. M. and Ingard, K. U. Theoretical Acoustics. Princeton University Press, 1987.

Moszynski, M. Fish target strength estimation using multiple echo statistics. Acoust. Phys., 48(2):201-208, 2002. 
Moszynski, M. and Hedgepeth, J. B. Using single-beam side-lobe observations of fish echoes for fish target strength and abundance estimation in shallow water. Aqua. Liv. Res., 13(5):379-383, 2000.

Mukai, T., Iida, K., Sakaguchi, K., and Abe, K. Estimations of squid target strength using a small cage and theoretical scattering models. In Proc. JSPS-DGHE Int. Sym. Fish. Sci. Trop. Area, pages 135-140, 2000.

Nero, R. W., Feuillade, C., Thompson, C. H., and Love, R. H. Near-resonance scattering from arrays of artificial fish swimbladders. J. Acoust. Soc. Am., 121(1): 132-143, 2007.

Nero, R. W., Thompson, C. H., and Jech, J. M. In situ acoustic estimates of the swimbladder volume of atlantic herring (Clupea harengus). ICES J. Mar. Sci., 61 (3):323-337, 2004.

O'Dor, R. Telemetered cephalopod energetics: swimming, soaring, and climping. Integr. Comp. Biol., 42(5):1065-1070, 2002.

Olshevskii, V. V. Characteristics of Sea Reverberation. Consultants Bureau, New York, 1967.

Ona, E. Physiological factors causing natural variations in acoustic target strength of fish. J. Mar. Biol. Assoc. U.K., 70(01):107-127, 1990.

Ona, E. An expanded target-strength relationship for herring. ICES J. Mar. Sci., 60 (3):493-499, 2003.

Onsrud, M. S. R., Kaartvedt, S., Rstad, A., and Klevjer, T. A. Vertical distribution and feeding patterns in fish foraging on the krill Meganyctiphanes norvegica. ICES J. Mar. Sci., 61(8):1278-1290, 2004.

Papoulis, A. Probability, Random Variables and Stochastic Processes. McGraw-Hill, 4th edition, 2002.

Pauly, D., Christensen, V., Gunette, S., Pitcher, T. J., Sumaila, U. R., Walters, C. J., Watson, R., and Zeller, D. Towards sustainability in world fisheries. Nature, 418 (6898):689-695, 2002.

Payne, A. G., Agnew, D. J., and Pierce, G. J. Trends and assessment of cephalopod fisheries. Fish. Res., 78(1):1-3, 2006.

Peterson, M. L., Clay, C. S., and Brandt, S. B. Acoustic estimates of fish density and scattering function. J. Acoust. Soc. Am., 60(3):618-622, 1976.

Quijano, J. E. and Dosso, S. E. Geoacoustic inversion from ambient noise data using a trans-dimensional bayesian approach. J. Acoust. Soc. Am., 130(4):2454, 2011.

Rebinsky, D. A. and Norris, A. N. Benchmarking an acoustic coupling theory for elastic shells of arbitrary shape. J. Acoust. Soc. Am., 98:2368-2368, 1995. 
Reeder, D. B., Jech, J. M., and Stanton, T. K. Broadband acoustic backscatter and high-resolution morphology of fish: Measurement and modeling. J. Acoust. Soc. Am., 116(2):747-761, 2004.

Revie, J., Weston, D. E., Jones, F. R. H., and Fox, G. P. Identification of fish echoes located at $65 \mathrm{~km}$ range by shore-based sonar. ICES J. Mar. Sci., 46(3):313-324, 1990.

Roberts, C. M. Deep impact: the rising toll of fishing in the deep sea. Trends Ecol. Evol., 17(5):242-245, 2002.

Roberts, P. L. D. and Jaffe, J. S. Multiple angle acoustic classification of zooplankton. J. Acoust. Soc. Am., 121(4):2060-2070, 2007.

Roberts, P. L. D. and Jaffe, J. S. Classification of live, untethered zooplankton from observations of multiple-angle acoustic scatter. J. Acoust. Soc. Am., 124(2):796$802,2008$.

Rodhouse, P. G. Managing and forecasting squid fisheries in variable environments. Fish. Res., 54(1):3-8, 2001.

Roper, C. F. E., Sweeney, M. J., and Nauen, C. E. Cephalopods of the World: An Annotated and Illustrated Catalogue of Species of Interest to Fisheries. United Nations Development Programme, Rome, 1984.

Rusby, J. S. M., Somers, M. L., Revie, J., McCartney, B. S., and Stubbs, A. R. An experimental survey of a herring fishery by long-range sonar. Mar. Biol., 22(3): 271-292, 1973.

Santos, M. B., Clarke, M. R., and Pierce, G. J. Assessing the importance of cephalopods in the diets of marine mammals and other top predators: problems and solutions. Fish. Res., 52(1-2):121-139, 2001.

Scharf, L. L. and McWhorter, L. T. Geometry of the Cramer-Rao bound. Sig. Proc., 31(3):301-311, 1993.

Scott, D. W. Multivariate Density Estimation: Theory, Practice, and Visualization. Wiley, 1992.

Seibel, B. A., Goffredi, S. K., Thuesen, E. V., Childress, J. J., and Robison, B. H. Ammonium content and buoyancy in midwater cephalopods. J. Exp. Mar. Biol. Ecol., 313(2):375-387, 2004.

Simmonds, J. and MacLennan, D. Fisheries Acoustics: Theory and Practice. WileyBlackwell, 2nd edition, 2006.

Smith, J. N., Ressler, P. H., and Warren, J. D. Material properties of euphausiids and other zooplankton from the Bering Sea. J. Acoust. Soc. Am., 128:2664, 2010. 
Stanton, T. K. Multiple scattering with applications to fish-echo processing. J. Acoust. Soc. Am., 73(4):1164-1169, 1983.

Stanton, T. K. Density estimates of biological sound scatterers using sonar echo peak PDFs. J. Acoust. Soc. Am., 78(5):1868, 1985a.

Stanton, T. K. Volume scattering: Echo peak PDF. J. Acoust. Soc. Am., 77(4): 1358-1366, 1985b.

Stanton, T. K. Sound scattering by cylinders of finite length. III. deformed cylinders. J. Acoust. Soc. Am., 86(2):691-705, 1989.

Stanton, T. K. and Chu, D. Non-Rayleigh echoes from resolved individuals and patches of resonant fish at $24 \mathrm{kHz}$. IEEE J. Oceanic Eng., 35(2):152-163, 2010.

Stanton, T. K. and Clay, C. Sonar echo statistics as a remote-sensing tool: Volume and seafloor. IEEE J. Oceanic Eng., 11(1):79-96, 1986.

Stanton, T. K. Broadband acoustic sensing of the ocean. J. Mar. Acoust. Soc. Jpn., 36(2):19-31, 2009.

Stanton, T. K. 30 years of advances in active bioacoustics: A personal perspective. Method. Oceanogr., 12:49-77, 2012.

Stanton, T. K. and Chu, D. Review and recommendations for the modeling of acoustic scattering by fluid-like elongated zooplankton: euphausiids and copepods. ICES J. Mar. Sci., 57(4):793-807, 2000.

Stanton, T. K. and Chu, D. On the acoustic diffraction by the edges of benthic shells. J. Acoust. Soc. Am., 116(1):239-244, 2004.

Stanton, T. K. and Chu, D. Calibration of broadband active acoustic systems using a single standard spherical target. J. Acoust. Soc. Am., 124(1):128-136, 2008.

Stanton, T. K., Chu, D., Wiebe, P. H., and Clay, C. S. Average echoes from randomly oriented random-length finite cylinders: Zooplankton models. J. Acoust. Soc. Am., 94(6):3463-3472, 1993a.

Stanton, T. K., Clay, C. S., and Chu, D. Ray representation of sound scattering by weakly scattering deformed fluid cylinders: Simple physics and application to zooplankton. J. Acoust. Soc. Am., 94(6):3454-3462, 1993b.

Stanton, T. K., Chu, D., and Wiebe, P. H. Sound scattering by several zooplankton groups. II. scattering models. J. Acoust. Soc. Am., 103(1):236-253, 1998a.

Stanton, T. K., Chu, D., Wiebe, P. H., Martin, L. V., and Eastwood, R. L. Sound scattering by several zooplankton groups. I. experimental determination of dominant scattering mechanisms. J. Acoust. Soc. Am., 103(1):225-235, 1998b. 
Stanton, T. K., Chu, D., Wiebe, P. H., Eastwood, R. L., and Warren, J. D. Acoustic scattering by benthic and planktonic shelled animals. J. Acoust. Soc. Am., 108(2): $535-550,2000$.

Stanton, T. K., Chu, D., Jech, J. M., and Irish, J. D. New broadband methods for resonance classification and high-resolution imagery of fish with swimbladders using a modified commercial broadband echosounder. ICES J. Mar. Sci., 67(2): 365-378, 2010.

Stanton, T. K., Sellers, C. J., and Jech, J. M. Resonance classification of mixed assemblages of fish with swimbladders using a modified commercial broadband acoustic echosounder at $16 \mathrm{kHz}$. Can. J. Fish. Aqua. Sci., 69(5):854-868, 2012.

Stanton, T., Chu, D., and Reeder, D. Non-Rayleigh acoustic scattering characteristics of individual fish and zooplankton. IEEE J. Oceanic Eng., 29(2):260-268, 2004.

Starr, R., Thorne, R., Rodhouse, P., Dawe, E., and O'Dor, R. Acoustic assessment of squid stocks. In Squid Recruitment Dynamics, pages 181-198. FAO Fish. Tech. Pap. No.376., Rome, 1998.

Stepnowski, A. and Moszynski, M. Inverse problem solution techniques as applied to indirect in situ estimation of fish target strength. J. Acoust. Soc. Am., 107(5): 2554-2562, 2000.

Sun, Y., Nash, R., and Clay, C. S. Acoustic measurements of the anatomy of fish at $220 \mathrm{kHz}$. J. Acoust. Soc. Am., 78(5):1772-1776, 1985.

Tarantola, A. Inverse Problem Theory and Methods for Model Parameter Estimation. Society for Industrial and Applied Mathematics, 2005.

Thompson, C. H. and Love, R. H. Determination of fish size distributions and areal densities using broadband low-frequency measurements. ICES J. Mar. Sci., 53(2): 197-201, 1996.

Trevorrow, M. V. and Pedersen, B. Detection of migratory herring in a shallow channel using 12-and 100-kHz sidescan sonars. Aqua. Liv. Res., 13(5):395-401, 2000 .

Turin, G. An introduction to matched filters. IRE Trans. Inf. Theory, 6(3):311-329, 1960.

Urick, R. J. Principles of Underwater Sound. McGraw-Hill, New York, 1983.

Van Trees, H. L. Detection, Estimation, and Modulation Theory. John Wiley, New York, 2001.

Ward, K. D. and Tough, R. J. A. Radar detection performance in sea clutter with discrete spikes. In $R A D A R$ 2002, pages 253-257. IEEE, 2002. 
Ward, K. D., Watts, S., and Tough, R. J. A. Sea Clutter: Scattering, the Kdistribution and Radar Performance, volume 20. IET, 2006.

Warren, J. D. and Smith, J. N. Density and sound speed of two gelatinous zooplankton: Ctenophore (Mnemiopsis leidyi) and lion's mane jellyfish (Cyanea capillata). J. Acoust. Soc. Am., 122(1):574-580, 2007.

Watts, S. and Wards, K. D., Eds. Special issue on Radar clutter. vol. 4, no.2, IET Radar, Sonar, and Navigation, 2010.

Welch, P. The use of fast Fourier transform for the estimation of power spectra: A method based on time averaging over short, modified periodograms. IEEE Trans. Audio Electroacoust., 15(2):70 - 73, 1967.

Weston, D. E. Sound propagation in the presence of bladder fish. In Albers, V. M., editor, Underwater acoustics, volume 2, pages 55-88. Plenum Press, New York, NY, 1967.

Wiebe, P. H., Greene, C. H., Stanton, T. K., and Burczynski, J. Sound scattering by live zooplankton and micronekton: Empirical studies with a dual-beam acoustical system. J. Acoust. Soc. Am., 88(5):2346-2360, 1990.

Wiebe, P. H., Chu, D., Kaartvedt, S., Hundt, A., Melle, W., Ona, E., and BattaLona, P. The acoustic properties of Salpa thompsoni. ICES J. Mar. Sci., 67(3): 583-593, 2010.

Wilhelmij, P. and Denbigh, P. A statistical approach to determining the number density of random scatterers from backscattered pulses. J. Acoust. Soc. Am., 76 (6):1810-1818, 1984.

Woillez, M., Ressler, P. H., Wilson, C. D., and Horne, J. K. Multifrequency species classification of acoustic-trawl survey data using semi-supervised learning with class discovery. J. Acoust. Soc. Am., 131(2):EL184-EL190, 2012.

Woolf, D. K. Bubbles and their role in gas exchange. In Liss, P. S. and R. A. Duce, E., editors, The Sea Surface and Global Change. Cambridge University Press, 1997.

Yasuma, H., Takao, Y., Sawada, K., Miyashita, K., and Aoki, I. Target strength of the lanternfish, Stenobrachius leucopsarus (family Myctophidae), a fish without an airbladder, measured in the bering sea. ICES J. Mar. Sci., 63(4):683-692, 2006.

Yasuma, H., Sawada, K., Takao, Y., Miyashita, K., and Aoki, I. Swimbladder condition and target strength of myctophid fish in the temperate zone of the northwest pacific. ICES J. Mar. Sci., 67(1):135-144, 2010.

Ye, Z. Low-frequency acoustic scattering by gas-filled prolate spheroids in liquids. $J$. Acoust. Soc. Am., 101:1945, 1997. 
Ye, Z. and Farmer, D. M. Acoustic scattering by fish in the forward direction. ICES J. Mar. Sci., 53(2):249-252, 1996.

Ye, Z. and Hoskinson, E. Low-frequency acoustic scattering by gas-filled prolate spheroids in liquids. II. comparison with the exact solution. J. Acoust. Soc. Am., 103(2):822-826, 1998. 